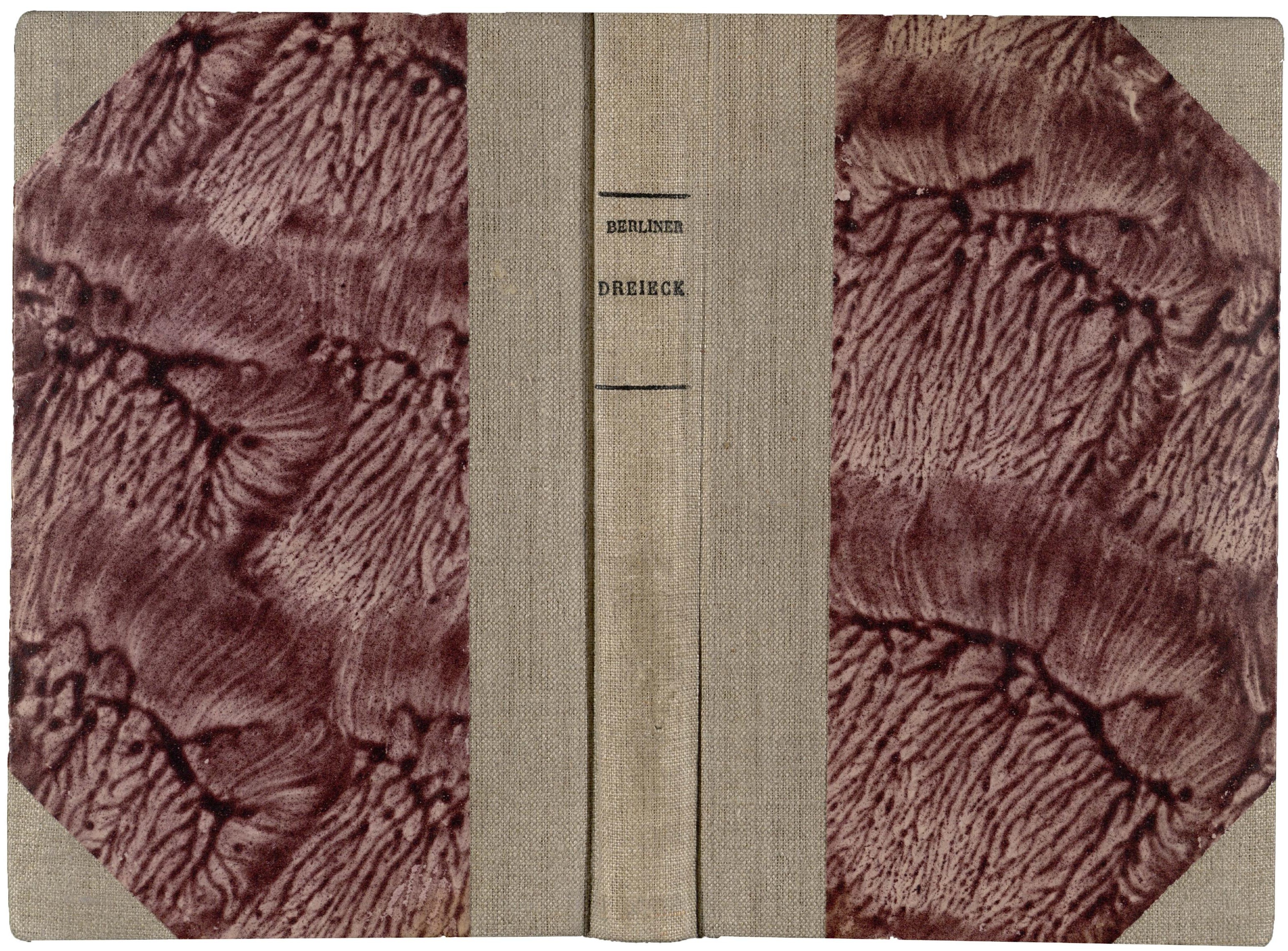




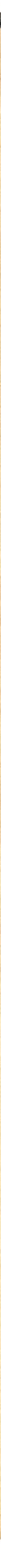



Iranownemu Tann Irof. I. Dichsteinowi

$Z$ srocunhiem od antora.

\title{
INVOLUTIONSSYSTEME
}

\section{IN DER EBENE DES DREIECKS}

\author{
GABINET MATEMATYCZNY \\ Inwarzystiwa Nauk'ontege Harszawskiego
}


www.rcin.org.pl 


\section{INVOLUTIONSSYSTEVE}

\section{IN DER EBENE DES DREIECKS}

VoN

\section{DR. PHIL. H. BERLINER}

PRIVATDOZENT AN DER UNIVERSITÄT BERN

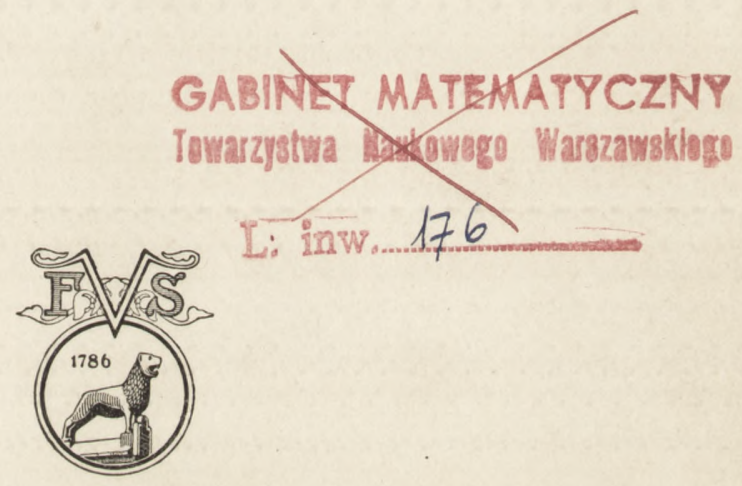

BRAUNSCHWEIG

DRUCK UND KOMMISSIONSVERLAG VON FRIEDR. VIEWEG \& SOHN

1914 
opis wr: 46704

Alle Rechte vorbehalten.

Copyright, 1914, by Friedr. Vieweg \& Sohn, Braunschweig, Germany.

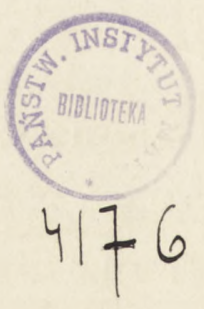

www.rcin.org.pl 


\section{VORWORT.}

In der vorliegenden Arbeit im ersten Abschnitt zeigte ich, daß in der Ebene eines Dreiecks um jeden Punkt und auf jeder Geraden Systeme von Involutionen vom Grade $2^{n}$ und $2^{n} .3$, wo $n$ jeden positiven ganzzahligen Wert annehmen soll, vom Dreieck erzeugt werden, und stellte einige Sätze über diese Involutionen auf. Dieselben Involutionen verleiten nun $\mathrm{zu}$ den Begriffen der $n$-ten Repräsentanten und $n$-ten Koinzidenz-Repräsentanten (eines mit seinem $n$-ten Repräsentanten zusammenfallenden Elementes), die im zweiten Abschnitt behandelt werden. Im dritten Abschnitt versuchte ich mit Hilfe der in den ersten beiden Abschnitten gefundenen Resultate auf einem neuen Wege zu den Kurven dritter Ordnung mit isoliertem Doppelpunkt (und zugleich zu den dualen Gebilden, zu den Kurven dritter Klasse mit isolierter Doppeltangente) zu gelangen, indem ich eine solche Kurve als Ort derjenigen Punkte definiere, die einem Punkte zugepaart sind in den auf den sämtlichen durch diesen Punkt gehenden Geraden von einem Dreieck erzeugten Punktinvolutionen zweiten Grades. Auf diesem Wege, ohne irgend einen Satz über Kurven dritter Ordnung vorauszusetzen, ergeben sich für die genannten Kurven fast alle bekannten visuellen Haupteigenschaften der allgemeinen Kurven dritter Ordnung und außerdem eine Reihe neuer Sätze und Konstruktionen, die nur für die genannten Kurven allein gelten. Dabei war vorausgesetzt, daß alle Ecken des zugrunde gelegten Dreiecks reell seien; und in der vorliegenden Arbeit ist auch durchweg nur von einem reellen Grunddreieck die Rede.

Es liegt nun aber nahe, daß, wenn ein solches Dreieck zugrunde gelegt wird, dessen eine Ecke reell und die beiden anderen konjugiert imaginär sind, durch die von einem solchen Dreieck erzeugten Involutionen zweiten Grades in der nämlichen Weise Kurven dritter Ordnung mit (reellem) Knotenpunkt entstehen werden. 
Es möge hier noch folgendes erwähnt werden.

Auch zu den Kurven vierter Ordnung mit dreifachem Punkt und zu den dualen Gebilden kann man mittels der von einem Dreieck erzeugten Involutionen zweiten Grades in einfacher Weise gelangen. Sind nämlich in der Ebene eines Dreiecks $A B C$ ein auf keiner Seite von $A B C$ liegender Punkt $P$ und eine weder durch $P$ noch durch eine der Ecken von $A B C$ gehende Gerade $l$ gegeben, und bestimmt man auf jedem Strahle von $P$ den Punkt, der dem Schnittpunkte des Strahles mit $l$ zugepaart ist in der von $A B C$ auf diesem Strahle erzeugten Punktinvolution zweiten Grades, so ist der Ort aller dieser Punkte eine Kurve vierter Ordnung und sechster, fünfter oder vierter Klasse, je nachdem $l$ keine Tangente, eine einfache Tangente oder eine Wendetangente der zu $P$ bezüglich $A B C$ zugehörigen Kurve dritter Ordnung $p^{3}$ (siehe Satz 33, S. 163) ist. Die so erzeugte Kurve vierter Ordnung hat $P$ zum dreifachen Punkt und die $P$ mit den Schnittpunkten von $l$ und $p^{3}$ verbindenden Geraden zu den Tangenten in $P$, ist $A B C$ umschrieben und geht durch die Berührungspunkte der beiden aus $P$ an den Polarkegelschnitt von $l$ (bezüglich $A B C$ ) gehenden Tangenten und durch diejenigen beiden Punkte des Polarkegelschnitts von $P$ (bezüglich $A B C$ ) hindurch, welche mit den beiden Schnittpunkten des letzteren Polarkegelschnitts und $l$ auf zwei Strahlen von $P$ liegen.

Wird nun der Punkt $P$ festgehalten, so entspricht jedem von $A, B, C$ verschiedenen Punkt $Q$ der Ebene von $A B C$ ein Punkt, der zu $Q$ zugepaart ist in der von $A B C$ auf der Geraden $P Q$ erzeugten Punktinvolution zweiten Grades; den Punkten einer beliebigen Geraden aber entsprechen im allgemeinen die Punkte einer Kurve vierter Ordnung. Auf diese Weise gelangt man zu einer eindeutigen involutorischen geometrischen Verwandtschaft vierten Grades. Hierauf gedenke ich demnächst näher einzugehen.

Die ersten 12 Paragraphen der vorliegenden Arbeit habe ich im Mai vorigen Jahres als Habilitationsschrift der philosophischen Fakultät der Universität Bern eingereicht. Die $\S \S 13$ und 14 sind erst nach erfolgter Habilitation hinzugefügt.

Bern, im Januar 1914.

Dr. H. Berliner. 


\section{Inhaltsverzeichnis.}

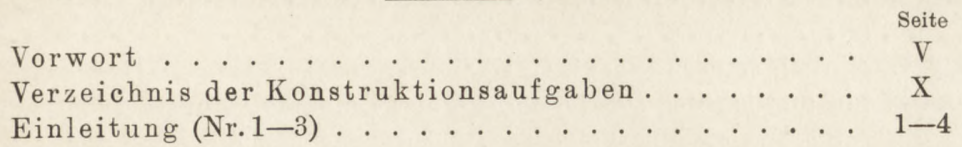

Erster Abschnitt.

\section{Vom Dreieck erzeugte Involutionen.}

$\S 1$. Die vom Dreieck im Gebilde erster Stufe erzeugten Involutionen zweiten und dritten Grades. Perspektivität der vom Dreieck um den Pol und auf der Polare erzeugten Involutionen (Nr. 4-6)

$\S$ 2. Die im Polarkegelschnitt induzierten Involutionen. Charakteristische Eigenschaft der vom Dreieck im Gebilde erster Stufe erzeugten Involution zweiten Grades. Repräsentanten. Projektivität der Involution zweiten Grades zum Gebilde der Repräsentanten. Perspektivität der vom Dreieck in verschiedenen Gebilden erster Stufe erzeugten Involutionen. Die durch das Dreieck in Gebilden erster Stufe festgelegten Sinne (Nr. 7-11)

§ 3. Einige Eigenschaften der im Polarkegelschnitt induzierten Involution dritten Grades. Charakteristische Eigenschaft der vom Dreieck im Gebilde erster Stufe erzeugten Involution dritten Grades (Nr. 12-18) . . . . . . . . . . . . . . . . .

$\S$ 4. Repräsentanten und Repräsentationsgruppen. Die vom Dreieck im Gebilde erster Stufe erzeugten Involutionen $\left(2^{n}\right)$-ten und $\left(2^{n} .3\right)$-ten Grades. Perspektivität dieser Involutionen in verschiedenen Gebilden. Involutorische Lage des Büschels der Repräsentanten um Pol zu der Punktreihe der Repräsentanten auf der Polare oder auf einer von drei ausgezeichneten Tangenten des Polarkegelschnitts der Polare (Nr. 19-23) . . . . .

\section{Zweiter Abschnitt.}

\section{Der Prozeß der Repräsentantenbildung.}

$\S 5$. Die $n$-ten Repräsentanten. Definition der Ordinatenwinkel. Eine Beziehung zwischen den Ordinatenwinkeln eines Elementes und seines $n$-ten Repräsentanten. Projektivität des Büschels der Repräsentanten um Pol zu der Punktreihe der Repräsentanten auf einer Tangente des Polarkegelschnitts der Polare. Eine Beziehung zwischen den ersten und zweiten Repräsentanten. Ein Zusammenhang zwischen den beiden Polen einer durch einen Punkt gehenden Geraden in bezug auf das Dreieck und 
den Polarkegelschnitt des Punktes. $n$-te, primitive $n$-te Koinzidenz-Repräsentanten und je ihre Anzahl. Eine zahlentheoretische Folgerung (Nr. 24-32). . . . . . . . . .

$\S$ 6. Vorkommen zweier Elemente eines Tripels der Involution dritten Grades unter den Repräsentanten des dritten Elementes des nämlichen Tripels. $n$-te Repräsentationen; $n$-te, primitive $n$-te Koinzidenz-Repräsentationen und je ihre Anzahl (Nr. 33-38) . . . . . . . . . . . . . . . . .

$\S 7$. Einteilung der Elemente im Gebilde erster Stufe in drei Klassen hinsichtlich des Prozesses der Repräsentantenbildung. Eine zahlentheoretische Folgerung. Einteilung der Elemente der ersten beiden Klassen in je zwei Arten (Nr. 39-45) . . . .

\section{Dritter Abschnitt.}

\section{Über eine neue, spezielle Erzeugungsart der Kurven dritter Ordnung mit isoliertem Doppelpunkt bzw. dritter Klasse mit isolierter Doppeltangente.}

$\S$ 8. Der Ort $p^{3}$ derjenigen Punkte, welche einem gegebenen Punkte $P$ zugepaart sind in den von $A B C$ auf den sämtlichen durch $P$ gehenden Geraden erzeugten Involutionen zweiten Grades, und das duale Gebilde $P^{3}$. Konstruktionen. Kriterium der Realität und der Lage der beiden weitern Schnittpunkte von $p^{3}$ mit einer durch einen gegebenen Punkt von $p^{3}$ gehenden Geraden und das duale für $P^{3}$ (Nr. 46-51) . . . . . . . .

$\S 9$. Kriterium der geradlinigen Lage dreier Punkte von $p^{3}$ und des Konvergierens dreier Strahlen von $P^{3}$ in einen Punkt. Zweites Kriterium der Realität und der Lage der beiden weitern Schnittpunkte von $p^{3}$ mit einer durch einen gegebenen Punkt von $p^{3}$ gehenden Geraden und das duale für $P^{3}$. Konstruktionen. Eine Reihe bekannter Sätze über die Kurven dritter Ordnung als Folgerungen aus den Aussagen I, II, III. Fine Relation zwischen den Ordinatenwinkeln dreier in einer Geraden liegender Punkte von $p^{3}$ (Nr.52-69). . . . . . .

$\S 10$. Der aus einem gegebenen Kegelschnitt abgeleitete Kegelschnitt. Kriterium der Realität und der Lage der vier weitern Schnittpunkte von $p^{3}$ mit einem durch zwei gegebene Punkte von $p^{3}$ gehenden Kegelschnitt und das duale für $P^{3}$. Kriterium dafür, daß sechs Punkte von $p^{3}$ auf einem Kegelschnitt liegen und das duale für $P^{3}$. Eine Relation zwischen den Ordinatenwinkeln von sechs auf einem Kegelschnitt liegenden Punkten von $p^{3}$. Einige Folgerungen. Konstruktionen, darunter solche von Oskulationskegelschnitten (Nr. 70-76).

$\S 11$. Nachweis dafür, daß jede Gerade mit $p^{3}$ drei Punkte gemein hat. Zusammenhang zwischen $p^{3}$ und $\boldsymbol{P}^{3}$. Konstruktion der drei Schnittpunkte einer Geraden mit $p^{3} . p^{3}$ und $P^{3}$ als Enveloppen von Kegelschnittsystemen. Zusammenhang zwi- 
schen der Tangente eines Punktes $P_{i}$ von $p^{3}$ und dem Berührungspunkt des entsprechenden Strahles $p_{i}$ von $P^{3}$. $p^{3}$ und $P^{3}$ als Erzeugnisse projektiver Gebilde. Identität der Kurven dritter Ordnung mit isoliertem Doppelpunkt bzw. dritter Klasse mit isolierter Doppeltangente mit $p^{3}$ bzw. $P^{3}$ (Nr. 77-82) . 160-171

$\S 12$. Kriterium der Realität und der Lage der drei Schnittpunkte von $p^{3}$ mit einer durch keine Ecke von $A B C$ gehenden Geraden. Zusammenhang zwischen den drei auf einer Geraden liegenden Punkten von $p^{3}$ und den drei durch $P$ gehenden Strahlen des jener Geraden in bezug auf $A B C$ zugehörigen Büschels dritter Ordnung. Konstruktionen, darunter solche der Asymptotenrichtungen und der Asymptoten selbst. Kriterium der Art von $p^{3}$ hinsichtlich ihrer unendlich fernen Punkte. Ein positives Kriterium der Realität und der Lage der drei Schnittpunkte von $p^{3}$ mit einer Geraden (Nr. 83-87) . . . . . . . . . . . . . .

$\S 13$. Der aus dem Polarkegelschnitt abgeleitete Kegelschnitt. Kriterium der Realität und der Lage der drei Schnittpunkte von $p^{3}$ mit einer beliebigen Geraden, und das duale für $P^{3}$. Direkte Konstruktion des abgeleiteten Kegelschnitts. Konstruktionen (Nr. 88-91) . . . . . . . . . . . . .

$\S 14$. Der einer Geraden $l$ mittels eines Büschels von abgeleiteten Kegelschnitten zugeordnete Kegelschnitt $L^{2}$. Direkte Bestimmung desselben Kegelschnitts. Ein Satz über die Punktetripel von $p^{3}$, die mit einem gegebenen Punkte in je einer Geraden liegen, und der duale für $P^{3}$. Kriterium dafür, daß ein Punkt $p^{3}$ angehört, und das duale für $P^{3}$. Kriterium dafür, daß zwei Punkte einander zugepaart sind in der von $A B C$ auf ihrer Verbindungsgeraden erzeugten Involution zweiten Grades, und das duale. Kriterium der Realität und der Lage der vier Schnittpunkte einer Geraden und der von $P^{3}$ eingehüllten Kurve bzw. der vier aus einem Punkte an $p^{3}$ gehenden Tangenten. Ein negatives Kriterium der Imaginärität der sämtlichen Schnittpunkte einer Geraden und der von $P^{3}$ eingehüllten Kurve, und dual. Kriterium dafür, daß die Tangenten in vier gegebenen Punkten von $p^{3}$ in einen Punkt konvergieren, und das duale für $P^{3}$. Sätze über die Strahlentripel, Strahlenpaare und Strahlenquadrupel von $P^{3}$, deren Berührungspunkte mit einem gegebenen Berührungspunkte, bzw. mit einem gegebenen Rückkehrpunkte von $P^{3}$, bzw. mit einem ganz beliebigen Punkte, der aber kein Berührungspunkt von $P^{3}$ ist, in je einer Geraden liegen. Die einem Punkte bezüglich der von $P^{3}$ eingehüllten Kurve vierter Ordnung zugeordnete Kurve dritter Ordnung. Konstruktionen der vier aus einem Punkte an $p^{3}$ gehenden Tangenten (Nr. 92-104) . . . . . . . 188-211 


\section{Verzeichnis der Konstruktionsaufgaben.}

1. Die drei Schnittpunkte einer beliebigen Geraden und $p^{3 *}$ ) zu finden: Aufgabe $23 \mathrm{im}$ Texte. . . . . . . . . . . . . . . . 165

2. Konstruktion . . . . . . . . . . . . . . . . . . . . . 183

2. Die drei Schnittpunkte einer Geraden $l$ und $p^{3}$ zu finden, wenn einer der drei durch $P$ gehenden Strahlen des $l$ zugehörigen Strahlenbüschels $L^{3}$ bekannt ist: Aufgabe $24 \mathrm{im}$ Texte. 2 Konstruktionen

3. Die beiden weiteren Schnittpunkte einer durch einen gegebenen Punkt von $p^{3}$ gehenden Geraden und $p^{3}$ zu finden: Aufgabe $1 \mathrm{im}$ Texte . . . . . . . . . . . . . . . . . 96

2. Konstruktion . . . . . . . . . . . . . . . . . 113

3. Konstruktion . . . . . . . . . . . . . . . . . . . . . . . . 184

4. Den dritten Schnittpunkt einer durch zwei gegebene Punkte von $p^{3}$ gehenden Geraden und $p^{3} \mathrm{zu}$ finden: Aufgabe 4 im Texte. 2 Konstruktionen. . . . . . . . . . . . . . . . . 113 3. Konstruktion . . . . . . . . . . . . . . . 185

5. Die durch zwei Punkte von $p^{3}$ gehende Gerade zu ziehen, wenn die Punkte selbst nicht gegeben sind, sondern die sie mit $P$ verbindenden Geraden: Aufgabe 6 im Texte . . . . . . . . . . . . .

6. Die Tangente in einem gegebenen Punkte von $p^{3}$ zu ziehen: Aufgabe 3 im Texte. . . . . . . . . . . . . . . . 98

2. und 3. Konstruktion . . . . . . . . . . . . . . 116

4. Konstruktion . . . . . . . . . . . . . . . . . 167

5., 6., 7. und 8. Konstruktion . . . . . . . . . . . . . . . . . . 186

7. Dieselbe Aufgabe, wenn der Punkt selbst nicht gegeben ist, sondern die ihn mit $P$ verbindende Gerade: Aufgabe 7 im Texte . . . . . 117

8. Die beiden aus einem $p^{3}$ angehörenden Punkte an $p^{3}$ gehenden Tangenten zu ziehen: Aufgabe 2 im Texte.......... 98

2. und 3. Konstruktion . . . . . . . . . . . . . . 115

4. Konstruktion ................... 186

*) Unter $p^{3}$ ist eine beliebige Kurve dritter Ordnung mit isoliertem Doppelpunkt zu verstehen, von der nur dieser Doppelpunkt, der etwa $P$ heißen möge, und diejenigen drei Punkte, deren Tangenten durch die Wendepunkte der Kurve gehen, gegeben zu sein brauchen (jene Tangenten selbst und ebenso die Wendepunkte brauchen aber nicht gegeben $\mathrm{zu}$ sein). 
9. Dieselhe Aufgabe, wenn der Punkt selbst nicht gegeben ist, sondern die ihn mit $P$ verbindende Gerade: Aufgabe 8 im Texte . . . . . 117

10. Den Tangentialpunkt eines Punktes von $p^{3}$ zu finden: Aufgabe 5 und $5 \mathrm{a}$ im Texte. 3 Konstruktionen . . . . . . . . . . . . . . 11 4., 5. und 6. Konstruktion sind in der 6., 7. und 8. Konstruktion der 6. Aufgabe (der 3. im Texte) enthalten . . . . . . . .

11. Die vier weiteren Schnittpunkte eines durch zwei gegebene Punkte von $p^{3}$ gehenden Kegelschnitts und $x^{3}$ zu finden: Aufgabe $10 \mathrm{im}$ Texte . . . . . . . . . . . . . . . . . .

12. Spezielle Konstruktion des sechsten Schnittpunktes eines durch fünf gegebene Punkte von $p^{3}$ gehenden Kegelschnitts und $p^{3}$ zu finden: Aufgabe $9 \mathrm{im}$ Texte ................. .

13. Denjenigen Kegelschnitt zu konstruieren, der durch drei gegebene Punkte von $p^{3}$ gehen und in einem derselben $p^{3}$ dreipunktig berühren soll: Aufgabe $11 \mathrm{im}$ Texte. . . . . . . . . . . .

14. Denjenigen Kegelschnitt zu konstruieren, der durch zwei gegebene Punkte von $p^{3}$ gehen und in einem derselben $p^{3}$ vierpunktig berühren soll: Aufgabe $12 \mathrm{im}$ Texte... . . . . . . . . . .

15. Denjenigen Kegelschnitt zu konstruieren, der durch zwei gewisse Punkte von $p^{3}$ gehen und in einem derselben $p^{3}$ fünfpunktig berühren soll: Aufgahe 13 im Texte.... . . . . . . . . .

16. Vier Punkte von $x^{3}$ seien gegeben; es soll ein solcher Punkt auf $p^{3}$ gefunden werden, der ein Berührungspunkt eines durch die vier gegebenen Punkte gehenden Kegelschnitts und $p^{3}$ sein soll: Aufgabe $14 \mathrm{im}$ Texte . . . . . . . . . . . . . . . . . .

17. Drei Punkte von $p^{3}$ seien gegeben; es soll ein solcher Punkt auf $p^{3}$ gefunden werden, der ein einfacher Berührungspunkt eines $p^{3}$ in einem der drei gegebenen Punkte (einfach) berührenden und durch die beiden übrigen Punkte gehenden Kegelschnitts und $p^{3}$ sein soll: Aufgabe 17 im Texte. 2 Konstruktionen . . . . . . . . .

18. Zwei Punkte von $p^{3}$ seien gegeben; es soll derjenige Punkt auf $p^{3}$ gefunden werden, der zusammen mit den gegebenen Punkten solche drei Punkte bildet, in denen $p^{3}$ einen und denselben Kegelschnitt berühren kann: Aufgabe 16 im Texte . . . . . . . . . . .

19. Ein Punkt von $p^{3}$ sei gegeben; es soll derjenige Punkt auf $p^{3}$ gefunden werden, der ein einfacher Berührungspunkt eines $p^{3}$ im gegebenen Punkte vierpunktig berührenden Kegelschnitts und $p^{3}$ ist: Aufgabe $15 \mathrm{im}$ Texte . . . . . . . . . . . . . .

20. Drei Punkte von $p^{3}$ seien gegeben; es soll ein solcher Punkt auf $p^{3}$ gefunden werden; in dem $p^{3}$ von einem durch die drei gegebenen Punkte gehenden Kegelschnitt dreipunktig berührt werden soll: Aufgabe $18 \mathrm{im}$ Texte. . . . . . . . . . . . . . . . .

21. Ein Punkt von $p^{3}$ sei gegeben; es soll ein solcher Punkt auf $p^{3}$ gefunden werden, der zugleich mit dem gegebenen Punkte je ein dreipunktiger Berührungspunkt von $p^{3}$ mit einem und demselben Kegelschnitt sein soll: Aufgabe 19 im Texte... . . . . . . . 
22. Zwei Punkte von $p^{3}$ seien gegeben; es soll ein solcher Punkt auf $p^{3}$ gefunden werden, in dem $p^{3}$ von einem durch die zwei gegebenen Punkte gehenden Kegelschnitt vierpunktig berührt werden soll: Aufgabe $20 \mathrm{im}$ Texte ............... 156

23. Ein Punkt von $p^{3}$ sei gegeben; es soll ein solcher Punkt auf $p^{3}$ gefunden werden, der ein vierpunktiger Berührungspunkt eines $p^{3}$ im gegebenen Punkte (einfach) berührenden Kegelschnitts und $p^{3}$ sein soll: Aufgabe 21 im Texte . . . . . . . . . . . . 158

24. Den Gegenpunkt von vier gegebenen Punkten von $p^{3}$ zu finden: Aufgabe 22 im Texte . . . . . . . . . . . . . . . . . . 159

25. Die vier aus einem beliebigen Punkte an $p^{3}$ gehenden Tangenten zu ziehen: Aufgabe $25 \mathrm{im}$ Texte. 2 Konstruktionen . . . . . . . . . 211

\section{Berichtigungen.}

S. 72 , Z. 6 v. u. und S. 73, Z. 1 v. o. lies: $(-1)^{p_{1}^{s-2} \cdot k}$ statt $(-1)^{p_{1}^{s-2 \cdot k}}$ S. 160 , Z. 1 v. o. lies: Satz 27 statt Satz 24. 


\section{GABINET MATEMATYCZNY Tewarzystwa Maukowgro Warszawstiagn}

\section{Einleitung.}

1. In der Ebene eines Dreiecks $A B C$, in dem den Ecken $A, B, C$ die Seiten $a, b, c$ gegenüberliegen, wird jedem auf keiner der Seiten liegenden Punkte $P$, als Pol, eine durch keine Ecke gehende Gerade $p$, als Polare, zugeordnet; und umgekehrt. Die Polare $p$ schneidet (Fig. 1) die Seiten $a, b, c$

Fig. 1.

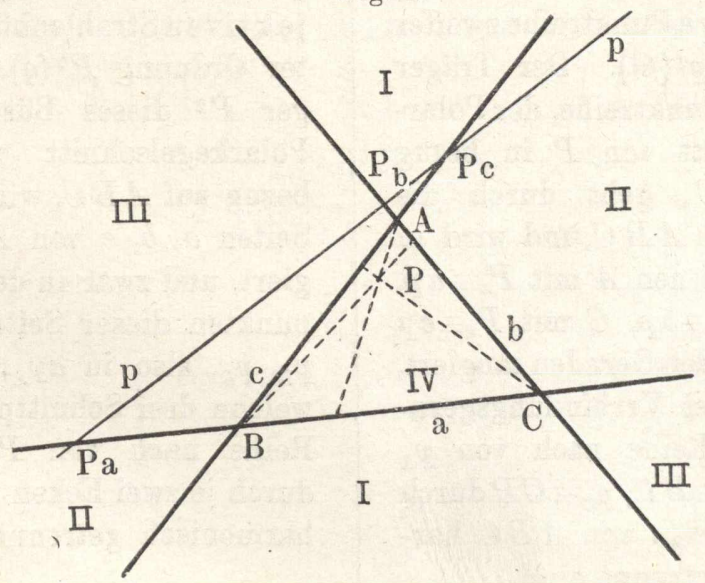

der Reihe nach in denjenigen Punkten $P_{a}, P_{b}, P_{c}$, welche bezw. von den Schnittpunkten der den Pol $P$ mit den Gegenecken verbindenden Geraden $P A \equiv p_{A}, P B \equiv p_{B}, P C \equiv p_{C}$ mit den Gegenseiten durch die je zwei übrigen Ecken harmonisch getrennt sind.

Eine unmittelbare Folge hiervon ist', daß von den sechs Strecken, welche von den Ecken des Dreiecks $A B C$ auf den Seiten desselben begrenzt werden, die drei, die von der Polare $p$ nicht getroffen werden, dasjenige Gebiet der Ebene begrenzen, innerhalb dessen der Pol $P$ liegt. Es werden also von den vier Gebieten (Fig. 1), in welche das Dreieck $A B C$ die Ebene teilt Berliner, Habilitationsschrift. 
und von welchen jedes von drei jener sechs Strecken begrenzt wird (wobei keine zwei dieser drei Strecken einer und derselben Seite angehören), nicht diejenigen drei Gebiete, in welche die Polare $p$ eindringt, sondern das vierte, in welches $p$ nicht eindringt, den Pol $P$ enthalten.

Allen auf einer Seite des Dreiecks $A B C$ liegenden Punkten ist die nämliche Seite, als Polare, zugeordnet und allen durch eine Ecke gehenden Geraden die nämliche Ecke, als Pol ${ }^{1}$ ).

$2^{2}$ ). Sind der auf keiner Seite von $A B C$ liegende Punkt $P$ und die durch keine Ecke gehende Gerade $p$ Pol und Polare in bezug auf $A B C$ und durchläuft

eine Gerade $g$ den Strahlenbüschel um $P$, so beschreibt der Pol $G$ von $g$ eine zu $P(g)$ projektive Punktreihe zweiter Ordnung $p^{2}(G)$. Der Träger $p^{2}$ dieser Punktreihe, der Polarkegelschnitt von $P$ in bezug auf $A B C$, geht durch die Ecken von $A B C$ und wird in diesen von den $A$ mit $P_{a} \equiv a p$, $B$ mit $P_{b} \equiv b p, C$ mit $P_{c} \equiv c p$ verbindenden Geraden tangiert, welche drei Verbindungsgeraden der Reihe nach von $p_{A}$ $\equiv A P, p_{B} \equiv B P, p_{C} \equiv C P$ durch je zwei Seiten von $A B C$ harmonisch getrennt sind. ein Punkt $Q$ die Punktreihe auf $p$, so beschreibt die Polare $q$ von $Q$ einen zu $p(Q)$ projektiven Strahlenbüschel zweiter Ordnung $P^{2}(q)$. Der Träger $P^{2}$ dieses Büschels, der Polarkegelschnitt von $p$ in bezug auf $A B C$, wird von den Seiten $a, b, c$ von $A B C$ tangiert, und zwar in den Schnittpunkten dieser Seiten mit $p_{A}$, $p_{B}, p_{C}$, also in $a p_{A}, b p_{B}, c p_{C}$, welche drei Schnittpunkte der Reihe nach von $P_{a}, P_{b}, P_{c}$ durch je zwei Ecken von $A B C$ harmonisch getrennt sind.

$P$ und $p$ sind Zentrum und Achse derjenigen beiden perspektiven Dreiecke, von denen das eine das Grunddreieck $A B C$ ist und das andere entweder das aus den Tangenten von $p^{2}$ in den Ecken von $A B C$ gebildete Dreieck, oder das aus den Berührungspunkten von $P^{2}$ mit den Seiten von $A B C$; ferner sind $P$ und $p$ Pol und Polare auch in bezug auf die beiden Polarkegelschnitte $p^{2}$ und $P^{2}$, und die Involutionen der in bezug auf $p^{2}$ und $P^{2}$

1) Vgl. meine Dissertation (von Bern): Theorie der Polaren in bezug auf Dreiecke, Nr. 6 (Leipzig 1912).

2) Hierzu vgl. Satz 4 (S. 46), Satz 13 und 14 (S. 61, 62) meiner Dissertation. 
konjugierten Elemente um $P$ und auf $p$ sind mit den rom Dreieck $A B C$ um $P$ und auf $p$ erzeugten elliptischen Involutionen zweiten Grades $(P)^{2}$ und $(p)^{2}$ (s. weiter unten Nr.4) identisch. Es liegt daher der Pol $P$ innerhalb der Polarkegelschnitte $p^{2}$ und $P^{2}$ und die Polare $p$ ganz außerhalb derselben; $p^{2}$ und $P^{2}$ berühren sich doppelt, ihre Berührungspunkte sind nämlich die beiden konjugiert-imaginären Doppelpunkte der Involution $(p)^{2}$ und ihre gemeinschaftlichen Tangenten die beiden konjugiertimaginären Doppelstrahlen der Involution $(P)^{2}$.

Weil kein Punkt innerhalb eines solchen der vier Dreiecksgebiete von $A B C$ liegen kann, in welches seine Polare in bezug auf $A B C$ eindringt (Nr. 1), so kann kein Punkt des Polarkegelschnitts $p^{2}$, welcher Punkt Pol einer durch $P$ gehenden Geraden ist, innerhalb des $P$ enthaltenden Dreiecksgebietes liegen; $p^{2}$ verläuft daher nur in den drei übrigen Dreiecksgebieten, und das vierte, $P$ enthaltende Dreiecksgebiet nebst seiner Begrenzung (nur die Ecken $A, B, C$ ausgenommen) gehört ganz dem Innern von $p^{2}$ an, da $P$, wie wir eben sahen, ein innerer Punkt von $p^{2}$ ist.

Weil ferner ein Kegelschnitt nebst allen von ihm eingeschlossenen Punkten in dem einen der beiden von irgend zwei seiner Tangenten gebildeten vollkommenen Winkel enthalten ist und mithin auch in dem einen der vier von irgend drei seiner Tangenten begrenzten Dreiecksgebiete, so muß der Polarkegelschnitt $P^{2}$, der von den Seiten von $A B C$ tangiert wird und von dem $P$ ein innerer Punkt ist, nebst allen von ihm eingeschlossenen Punkten in demjenigen der vier Dreiecksgebiete von $A B C$ enthalten sein, innerhalb dessen $P$ liegt; die drei übrigen Dreiecksgebiete gehören daher ganz dem Äußern von $P^{2}$ an, und sämtliche Tangenten von $P^{2}$, also alle Polaren der auf $p$ liegenden Punkte, müssen in das vierte, $P$ enthaltende Dreiecksgebiet eindringen.

Von den beiden Polarkegelschnitten $p^{2}$ und $P^{2}$ liegt demnach der letztere ganz innerhalb des ersteren.

Ist aber $P$ ein auf einer Seite von $A B C$, etwa auf der Seite $a$, liegender, jedoch von $B$ und $C$ verschiedener Punkt und durchläuft eine Gerade $g$
Ist $l$ irgendeine durch eine Ecke von $A B C$, etwa durch die Ecke $A$, gehende, jedoch von den Dreieckseiten $b$ und $c$ verschiedene Gerade und durch- 
den Strahlenbüschel um $P$, so beschreibt der Pol $G$ von $g$ eine zu $P(g)$ projektive Punktreihe erster Ordnung, deren Träger durch die Ecke $A$ geht und von $P$ durch die Dreieckseiten $b$ und $c$ harmonisch getrennt ist (diese Punktreihe liegt zu $P(g)$ weder perspektiv noch involutorisch). Außer dieser Punktreihe kann noch auch die von der Dreieckseite $a$ getragene Punktreihe als Erzeugnis der Pole des Strahlenbüschels $P(g)$ aufgefaßt werden, da, wenn $g$ nach $a$ kommt, jeder Punkt von $a$ als der Pol $G$ von $g$ angesehen werden kann; der Polarkegelschnitt $p^{2}$ von $P$ in bezug auf $A B C$ artet also dann in ein Geradenpaar aus, nämlich in die Dreieckseite $a$ und den Träger der ersteren Punktreihe.

DerPolarkegelschnitteiner Ecke von $A B C$ in bezug auf dieses Dreieck artet in dasjenige Geradenpaar aus, das aus den beiden durch jene Ecke gehenden Dreieckseiten besteht. läuft ein Punkt $Q$ die Punktreihe auf $l$, so beschreibt die Polare $q$ von $Q$ einen zu $l(Q)$ projektiven Strahlenbüschel erster Ordnung, dessen Grundpunkt auf der Dreieckseite $a$ liegt und von $l$ durch $B$ und $C$ harmonisch getrennt ist (dieser Strahlenbüschel liegt zu $l(Q)$ weder perspektiv noch involutorisch). Außer diesem Strahlenbüschel kann noch auch der Strahlenbüschel um $A$ als Erzeugnis der Polaren der Punktreihe $l(Q)$ aufgefaßt werden, da, wenn $Q$ nach $A$ kommt, jede durch $A$ gehende Gerade als die Polare $q$ von $Q$ angesehen werden kann; der Polarkegelschnitt $L^{2}$ von $l$ in bezug auf $A B C$ artet also dann in ein Punktepaar aus, nämlich in die Ecke $A$ und den Grundpunkt des ersteren Strahlenbüschels.

DerPolarkegelschnitteiner Seite von $A B C$ in bezug auf dieses Dreieck artet in dasjenige Punktepaar aus, das aus den beiden auf jener Seite liegenden Ecken von $A B C$ besteht.

$\left.3{ }^{1}\right)$. Wir setzen nun ein für allemal voraus, daß alle drei Ecken des Grunddreiecks $A B C$ reell seien, und in der Folge wird nur von einem reellen Grunddreieck die Rede sein. Der Definition von Pol und Polare zufolge wird in bezug auf ein

1) Diese Nummer darf übergangen werden, wenn man sich nur auf das Reelle beschränken will. 
solches Dreieck jedem reellen Punkte, als Pol, eine reelle Gerade, als Polare, entsprechen und jedem auf keiner Seite des Dreiecks liegenden imaginären Punkte eine durch keine Ecke desselben gehende imaginäre Gerade, und umgekehrt; nur ein auf einer Dreieckseite liegender imaginärer Punkt wird eine reelle Polare besitzen, nämlich jene Dreieckseite selbst, und nur eine durch eine Ecke gehende imaginäre Gerade einen reellen Pol, nämlich jene Ecke selbst.

Ist nun ein imaginärer Punkt irgendeiner, von den Seiten des Grunddreiecks $A B C$ verschiedenen reellen Geraden $l$ durch die ihn darstellende reelle elliptische Punktinvolution auf l, verbunden mit einem bestimmten Sinne auf $l$ gegeben ${ }^{1}$ ), so induziert diese elliptische Punktinvolution, weil die Punktreihe der Pole auf $l$ zu dem Strahlenbüschel erster oder zweiter Ordnung (je nachdem $l$ durch eine Ecke des Grunddreiecks $A B C$ geht oder nicht) der Polaren projektiv ist (Nr. 2), im Büschel der Polaren der Punkte von $l$ eine elliptische Strahleninvolution, in der die Strahlenpaare aus je zwei Polaren eines Punktepaares der Punktinvolution auf $l$ bestehen; einer der beiden konjugiertimaginären Doppelstrahlen dieser induzierten elliptischen Strahleninvolution, und zwar der mit demjenigen Sinne im Büschel der Polaren verbundene, welchem Sinne der mit dem gegebenen imaginären Punkte verbundene Sinn in der projektiven Punktreihe der Pole auf $l$ entspricht, wird dann die Polare des gegebenen imaginären Punktes in bezug auf $A B C$ sein.

Wir haben also:

Ist ein auf irgendeiner, von den Seiten von $A B C$ verschiedenen reellen Geraden $g$ liegender imaginärer Punkt $P$ durch die ihn darstellende reelle, elliptische Punktinvolution verbunden mit einem bestimmten Sinne auf $g$ gegeben, so wird die imaginäre Polare $p$ von $P$ durch diejenige reelle elliptische Tangenteninvolution
Ist eine durch irgendeinen, von den Ecken von $A B C$ verschiedenen reellen Punkt $Q$ gehende imaginäre Gerade $l$ durch die sie darstellende reelle elliptische Strahleninvolution, verbunden mit einem bestimmten Sinne im Büschel um $Q$ gegeben, so wird der imaginäre Pol $L$ von $l$ durch diejenige reelle elliptische Punktinvolu-

$\left.{ }^{1}\right)$ Siehe v. Staudt, Beiträge zur Geometrie der Lage, Nr.116, S. 76. 
um den Polarkegelschnitt $G^{2}$ von $g$ bzw., wenn $g$ durch eine Ecke von $A B C$ geht, durch diejenige reelle elliptische Strahleninvolution um den von $g$ durch die beiden übrigen Ecken von $A B C$ harmonisch getrennten Punkt, deren Paare aus je zwei Polaren eines Punktepaares der Punktinvolution auf $g$ bestehen, und durch denjenigen Sinn im Tangentenbüschel um $G^{2}$ bzw. im Strahlenbüschel um den von $g$ durch die zwei Ecken harmonisch getrennten Punkt, welchem Sinne der mit $P$ verbundene Sinn in der projektiven Punktreihe der Pole auf $g$ entspricht, dargestellt.

Diese reelle Darstellung der imaginären Polare $p$ von $P$ ist nur dann die einfachste, wenn $g$ durch eine Ecke von $A B C$ geht; ist dies aber nicht der Fall, so ist die einfachste reelle Darstellung von $p$ durch die reelle elliptische Involution der in bezug auf $G^{2}$, den Polarkegelschnitt von $g$, konjugierten Strahlen um das Zentrum jener Tangenteninvolution um $G^{2}$ und durch denjenigen Sinn im Büschel um dieses Zentrum, welcher zu dem mit dem imaginären Punkte $P$ verbundenen Sinne auf (der ganz außerhalb $G^{2}$ verlaufenden (Nr. 2) und also nicht durch das innerhalb tion auf dem Polarkegelschnitt $q^{2}$ von $Q$ bzw., wenn $Q$ auf einer Seite von $A B C$ liegt, auf der von $Q$ durch die beiden anderen Seiten von $A B C$ harmonisch getrennten Geraden, deren Punktepaare aus je zwei Polen eines Strahlenpaares der Strahleninvolution um $Q$ bestehen, und durch denjenigen Sinn auf dem Polarkegelschnitt $q^{2}$ bzw. auf der von $Q$ durch die zwei Seiten harmonisch getrennten Geraden, welchem Sinne der mit $l$ verbundene Sinn in dem projektiven Büschel der Polaren um $Q$ entspricht, dargestellt.

\section{Diese reelle Darstellung} des imaginären Pols $L$ von $l$ ist nur dann die einfachste, wenn $Q$ auf einer Seite von $A B C$ liegt; ist dies aber nicht der Fall, so ist die einfachste reelle Darstellung von $L$ durch die reelle elliptische Involution der in bezug auf $q^{2}$, den Polarkegelschnitt von $Q$, konjugierten Punkte auf der Achse jener Punktinvolution auf $q^{2}$ und durch denjenigen Sinn auf dieser Achse, welcher zu dem mit der imaginären $\mathrm{Ge}$ raden $l$ verbundenen Sinne im Büschel um (den innerhalb $q^{2}$ und also nicht auf der ganz außerhalb $q^{2} \quad$ verlaufenden 
$G^{2}$ liegende Zentrum jener elliptischen Tangenteninvolution um $G^{2}$ gehenden Geraden) $g$

Achse jener elliptischen Punktinvolution auf $q^{2}$ liegenden Punkt) $Q$

entgegengesetzt ist (wobei ein durch irgend drei Strahlen $x, y, z$ eines Büschels erster Ordnung festgelegter Sinn und ein durch irgend drei Punkte $T, U, V$ einer durch den Grundpunkt des Büschels nicht gehenden Geraden festgelegter Sinn als einander entgegengesetzt angesehen werden, wenn der Sinn der Spuren von $x, y, z$ auf der Geraden zum Sinne $T U V$ entgegengesetzt ist) ${ }^{1}$ ), wenn mit

der aus dem Zentrum jener Tangenteninvolution um $G^{2}$ an $G^{2}$ gehenden imaginären Tangente $p$ derjenige Sinn im Büschel um das Zentrum verbunden wird, welcher Sinn auf jeder Tangente von $G^{2}$ den nämlichen Sinn hervorruft (durch den Schnitt dieser Tangente mit dem Büschel um das innerhalb $G^{2}$ liegende Zentrum) ,wie der mit $p$ im Büschel der Tangenten von $G^{2}$ verbundene Sinn.

Denn die imaginären Doppelpunkte jener elliptischen Punktinvolution auf $q^{2}$ rechts liegen bekanntlich auf der Involutionsachse und sind zugleich die Doppelpunkte der Involution der in bezug auf $q^{2}$ konjugierten Punkte auf dieser Achse. Ferner muß, wie die Anschauung lehrt, ein auf $q^{2}$ fest gewählter Sinn auf dieser ganz außerhalb $q^{2}$ verlaufenden Achse durch Projektion von einem jeden auf $q^{2}$ liegenden Punkte aus den nämlichen Sinn hervorrufen wie durch Projektion von einem jeden innerhalb $q^{2}$ liegenden Punkte aus, insbesondere also von dem innerhalb $q^{2}$ liegenden Punkte $Q$ aus. Wenn aber eine Gerade $r$ den Strahlenbüschel um $Q$ in dem mit der imaginären Geraden $l$ verbundenen Sinne beschreibt und ihr Pol $R$ also den Polarkegelschnitt $q^{2}$

1) Siehe v. Staudt, Beiträge zur Geometrie der Lage, Nr. 48, S. 30. 
von $Q$ in dem mit $L$, dem imaginären Pole von $l$, verbundenen Sinne, so dreht sich, wie wir später (Nr.26) zeigen werden, die von $Q$ aus den Pol $R$ projizierende Gerade um $Q$ in demjenigen Sinne, welcher zu dem mit $l$ verbundenen Sinne entgegengesetzt ist; mithin ruft der mit $L$ auf $q^{2}$ verbundene Sinn durch Projektion von irgendeinem auf $q^{2}$ liegenden Punkte aus ebenso wie durch Projektion von $Q$ aus auf der Achse jener Punktinvolution denjenigen Sinn hervor, welcher zu dem mit $l$ im Strahlenbüschel um $Q$ verbundenen Sinne entgegengesetzt ist.

Wenn also $g$ durch keine Ecke von $A B C$ geht, so ist die Polare $p$ von $P$ eine der beiden aus demZentrum der genannten Tangenteninvolution um $G^{2}$ an $G^{2}$ gehenden konjugiertimaginären Tangenten, und zwar die mit demjenigen Sinne im Strahlenbüschel um das Zentrum verbundene imaginäre Tangente, welcher Sinn zu dem mit $P$ auf $g$ verbundenen Sinne entgegengesetzt ist.
Wenn also $Q$ auf keiner Seite von $A B C$ liegt, so ist $\operatorname{der} \operatorname{Pol} L$ von $l$ einer der beiden konjugiert-imaginären Schnittpunkte von $q^{2}$ mit der Achse der genannten Punktinvolution auf $q^{2}$, und zwar der mit demjenigen Sinne auf der Achse verbundene imaginäre Schnittpunkt, welcher Sinn zu dem mit $l$ im Strahlenbüschel um $Q$ verbundenen Sinne entgegengesetzt ist. 


\section{Vom Dreieck erzeugte Involutionen.}

\section{$\S 1$.}

4. In der Ebene des Dreiecks $A B C \equiv a b c$ werden durch das letztere

um jeden auf keiner Seite des Dreiecks liegenden Punkt $P$ drei Strahlen bestimmt, nämlich die drei Ecktransversalen $p_{A}, p_{B}$ und $p_{C}$, welche $P$ der Reihe nach mit den Ecken $A, B$ und $C$ verbinden. Durch diese drei Strahlen auf jeder durch keine Ecke des Dreiecks gehenden Geraden $p$ drei Punkte bestimmt, nämlich die drei Punkte $P_{a}$, $P_{b}$ und $P_{c}$, in welchen $p$ der Reihe nach von den Seiten $a$, $b$ und $c$ geschnitten wird. Durch diese drei Punkte

wird nun eine ternäre zyklische Projektivität um $P$ festgelegt, nämlich

$$
p_{A} p_{B} p_{C} \pi p_{B} p_{C} p_{A} \text {. }
$$

auf $p$ festgelegt, nämlich

$$
P_{a} P_{b} P_{c} \pi P_{b} P_{c} P_{a} \text {. }
$$

Wir wollen nun die beiden aus dieser zyklischen Projektivität hervorgehenden Involutionen, nämlich: die gemeine elliptische Involution, in welcher die Paare aus je einem Elemente eines Zyklus der Projektivität und dem zu ihm vierten harmonischen in bezug auf die beiden anderen Elemente desselben Zyklus bestehen ${ }^{1}$ ), und deren Doppelelemente mit den Koinzidenzelementen der Projektivität identisch sind, in welcher Involution also

$$
p_{A} p_{A}^{\prime}, p_{B} p_{B}^{\prime}, p_{C} p_{C}^{\prime}
$$

drei Strahlenpaare bilden, wo $p_{A}^{\prime}$ von $p_{A}$ durch $p_{B}$ und $p_{C}$ und durch $p_{B}^{\prime}$ und $p_{C}^{\prime}, p_{B}^{\prime}$ von

$$
P_{a} P_{a}^{\prime}, P_{b} P_{b}^{\prime}, P_{c} P_{c}^{\prime}
$$

drei Punktepaare bilden, wo $P_{a}^{\prime}$ von $P_{a}$ durch $P_{b}$ und $P_{c}$ und durch $P_{b}^{\prime}$ und $P_{c}^{\prime}, P_{b}^{\prime}$ von

1) Vgl. Sturm, Geometrische Verwandsehaften, Bd.I, Nr. 75. 
$p_{B}$ durch $p_{C}$ und $p_{A}$ und durch $p_{C}^{\prime}$ und $p_{A}^{\prime}, p_{C}^{\prime}$ von $p_{C}$ durch $p_{A}$ und $p_{B}$ und durch $p_{A}^{\prime}$ und $p_{B}^{\prime}$ harmonisch getrennt sind,
$P_{b}$ durch $P_{c}$ und $P_{a}$ und durch $P_{c}^{\prime}$ und $P_{a}^{\prime}, P_{c}^{\prime}$ von $P_{c}$ durch $P_{a}$ und $P_{b}$ und durch $P_{a}^{\prime}$ und $P_{b}^{\prime}$ harmonisch getrennt sind,

und die Involution dritten Grades, welche von den Zyklen derselben Projektivität gebildet wird, die vom Dreieck $A B C$

um den Punkt $P$ erzeugten Strahleninvolutionen zweiten und dritten Grades nennen und sie mit $(P)^{2}$ und $(P)^{3}$ bezeichnen. auf der Geraden $p$ erzeugten Punktinvolutionen zweiten und dritten Grades nennen und sie mit $(p)^{2}$ und $(p)^{3}$ bezeichnen.

Die Punktinvolution $(p)^{3}$ wird aus den Punkten, aus denen $(p)^{2}$ durch je eine rechtwinklige Strahleninvolution projiziert wird, durch je eine Involution der regelmäßigen Dreistrahlen projiziert, d. h. durch je eine solche Involution dritten Grades, deren Tripel aus je drei Strahlen, welche miteinander Winkel von $60^{\circ}$ bilden, bestehen $\left.{ }^{1}\right)$. Dasselbe gilt, wenn an Stelle von $(p)^{3}$ und $(p)^{2}$ die Schnitte von $(P)^{3}$ und $(P)^{2}$ durch irgendeine Gerade treten.

Die beiden Involutionen $(P)^{2}$ und $(P)^{3}$ und ebenso $(p)^{2}$ und $(p)^{3}$ sind hiernach in folgender Weise untereinander verbunden. Die in der Involution zweiten Grades den Elementen irgendeines Tripels der Involution dritten Grades zugepaarten Elemente bilden das zu jenem Tripel harmonische in der letzteren Involution; und zwar werden die beiden Tripel den nämlichen Sinn haben. Ermittelt man zu irgendeinem Paare in der (elliptischen) Involution zweiten Grades diejenigen beiden immer einzig vorhandenen Paare, welche zusammen ebenso harmonisch beschaffen sind wie die drei Paare $\left.p_{A} p_{A}^{\prime}, p_{B} p_{B}^{\prime}, p_{C} p_{C}^{\prime}{ }^{2}\right)$, so bilden die Elemente dieser drei Paare auf eine Weise zwei Tripel in der Involution dritten Grades, und zwar zwei harmonische. Überhaupt, wenn sechs Elemente drei Paare in der Involution zweiten Grades und zugleich zwei Tripel in der Involution dritten Grades bilden, müssen die drei Paare ebenso harmonisch beschaffen sein wie $p_{A} p_{A}^{\prime}$, $p_{B} p_{B}^{\prime}, p_{C} p_{C}^{\prime}$ und aus dem ersten und vierten bzw. zweiten und fünften bzw. dritten und sechsten (in der natürlichen Anordnung

1) Siehe Sturm, Geometrische Verwandschaften, Bd. I, Nr. 142.

2) Siehe meine Dissertation, IV. Abschnitt, Nr. 15. 
in einem der beiden Sinne im Gebilde) Elemente bestehen und die zwei Tripel zueinander harmonisch sein und aus dem ersten, dritten und fünften bzw. zweiten, vierten und sechsten Elemente bestehen.

Anmerkung. Wenn in einem Gebilde erster Stufe zwei ebenso wie $(P)^{2}$ und $(P)^{3}$ untereinander verbundene Involutionen zweiten und dritten Grades sich vorfinden und eine von ihnen mit der von $A B C$ im Gebilde erzeugten Involution gleichen Grades identisch ist, so muß dasselbe, wie man leicht einsieht, auch von der zweiten Involution gelten.

5. Sind der Punkt $P$ und die Gerade $p$ Pol und Polare in bezug auf $A B C$, so sind dann und nur dann die von $A B C$ um $P$ erzeugten Strahleninvolutionen $(P)^{2}$ und $(P)^{3}$ zu den auf $p$ erzeugten Punktinvolutionen $(p)^{2}$ und $(p)^{3}$ derart perspektiv, daß jedes der beiden von $A B C$ direkt herrührenden Tripel $p_{A} p_{B} p_{C}$ und $P_{a} P_{b} P_{c}$ von $(P)^{3}$ bzw. $(p)^{3}$ mit dem zum anderen Tripel harmonischen, also mit dem in der Involution zweiten Grades zum anderen zugepaarten Tripel inzidiert; und zwar inzidiert jedes Element der ersteren beiden Tripel mit demjenigen Elemente, welches in der Involution zweiten Grades zu dem dem ersteren Elemente dual gegenüberstehenden zugepaart ist, so daß $p_{A}, p_{B}$, $p_{c}, p_{A}^{\prime}, p_{B}^{\prime}, p_{C}^{\prime}$ der Reihe nach durch $P_{a}^{\prime}, P_{b}^{\prime}, P_{c}^{\prime}, P_{a}, P_{b}, Y_{c}$ gehen ${ }^{1}$ ).

6. Um einen auf einer Seite von $A B C$ liegenden, aber von den Ecken verschiedenen Punkt

Auf einer durch eine Ecke von $A B C$ gehenden, aber von den Seiten verschiedenen Geraden

wird von $A B C$ nur eine parabolische Involution erzeugt, in welcher allen Strahlen die durch den Punkt gehende Seite zugeordnet ist. in welcher allen Punkten die auf der Geraden liegende Ecke zugeordnet ist.

Denn in diesem Falle sind zwei der drei durch den Punkt gehenden Ecktransversalen von $A B C$ in der Seite zusammengefallen bzw. zwei der drei Schnittpunkte der Geraden mit den Seiten von $A B C$ in der Ecke.

1) Siehe meine Dissertation, Nr.5, S. 15. 
Die beiden von $A B C$ um einen seiner Ecken auf einer seiner Seiten erzeugten Involutionen zweiten und dritten Grades sind in der Weise unbestimmt, daß jede beliebige durch diese Ecke gehende Gerade zusammen mit den beiden Seiten als die drei durch diesen Punkt (Ecke) gehenden Ecktransversalen von $A B C$ jeder beliebige auf dieser Seite liegende Punkt zusammen mit den beiden Ecken als die drei Schnittpunkte dieser Geraden (Seite) mit den Seiten von $A B C$

und somit als die die ternäre zyklische Projektivität und die beiden aus dieser hervorgehenden Involutionen bestimmenden angesehen werden können.

\section{§ 2.}

7. Die beiden von $A B C$ um einen auf keiner Dreieckseite liegenden Punkt $P$ erzeugten Strahleninvolutionen $(P)^{2}$ $(P)^{3}$ induzieren nun in dem Polarkegelschnitt $p^{2}$ von $P$, weil der Büschel der Polaren um $P$ zu der Punktreihe der Pole auf $p^{2}$ projektiv ist (Nr. 2), zwei ebenso wie $(P)^{2}$ und $(P)^{3}$ untereinander verbundene Punktinvolutionen $\left(p^{2}\right)^{2}$ und $\left(p^{2}\right)^{3}$, in welchen die Gruppen aus je den Polen ${ }^{1}$ ) der in $(P)^{2}$ bzw. $(P)^{3}$ Gruppen bildenden Strahlen gebildet werden und welche Punktinvolutionen, weil die Pole der drei durch $P$ gehenden Ecktransversalen $p_{A}$, $p_{B}, p_{C}$ die Ecken $A, B, C$ sind, aus der ternären zyklischen Projektivität

\section{$A B C \bar{\wedge} B C A$}

auf $p^{2}$ hervorgehen ebenso wie $(P)^{2}$ und $(P)^{3}$ aus

$$
p_{A} p_{B} p_{C} \pi p_{B} p_{C} p_{A} \text {. }
$$

Dual induzieren die beiden Punktinvolutionen $(p)^{2}$ und $(p)^{3}$, deren Träger die Polare $p$ von $P$ ist, im Polarkegelschnitt $P^{2}$ von $p$ zwei Tangenteninvolutionen $\left(P^{2}\right)^{2}$ und $\left(P^{2}\right)^{3}$, welche letztere aus der ternären zyklischen Projektivität

$$
a b c \pi b c a
$$

1) Hier und überall im Folgenden, wo von Pol und Polare schlechthin die Rede ist, sollen die in bezug auf das Dreieck $A B C$ zugehörigen verstanden werden. 
hervorgehen und deren Gruppen aus je den Polaren der in $(p)^{2}$ bzw. in $(p)^{3}$ Gruppen bildenden Punkte gebildet werden.

Von den beiden ein Punktepaar in $\left(p^{2}\right)^{2}$ bildenden Polen des Strahlenpaares $p_{A} p_{A}^{\prime}$ von $(P)^{2}$ ist der eine, nämlich der Pol von $p_{A}$ die auf diesem liegende Ecke $A$ und der andere, nämlich der Pol von $p_{A}^{\prime}$ muß, weil $p_{A}^{\prime}$ von $p_{A}$ durch $p_{B}$ und $p_{C}$, also durch die Ecken $B$ und $C$ harmonisch getrennt ist, einer der auf $p_{A}$ liegenden Punkte und also der zweite Schnittpunkt von $p_{A}$ mit $p^{2}$ sein; somit ist $p_{A}$ die Verbindungsgerade dieses Punktepaares von $\left(p^{2}\right)^{2}$. Ebenso ist $p_{B}$ die Verbindungsgerade desjenigen Punktepaares von $\left(p^{2}\right)^{2}$, welches von den Polen des Strahlenpaares $p_{B} p_{B}^{\prime}$ von $(P)^{2}$ gebildet wird. Mithin ist $P$, der Schnittpunkt von $p_{A}$ und $p_{B}$, das Zentrum und $p$, die in bezug auf $A B C$ und $p^{2}$ gemeinsame Polare von $P$ (Nr.2), die Achse der induzierten Punktinvolution $\left(p^{2}\right)^{2}$. Dual sind $P$ und $p$ Zentrum und Achse auch der induzierten Tangenteninvolution $\left(P^{2}\right)^{2}$.

Hieraus erhellt:

Die Verbindungsgerade der Pole zweier Geraden, welche letztere in der von $A B C$ um ihren Schnittpunkt $P$ erzeugten Strahleninvolution $(P)^{2}$ einander zugepaart sind, geht durch $P$; und umgekehrt.
Der Schnittpunkt der Polaren zweier Punkte, welche letztere in der von $A B C$ auf ihrer Verbindungsgeraden $p$ erzeugten Punktinvolution $(p)^{2}$ einander zugepaart sind, liegt auf $p$; und umgekehrt.

Oder mit anderen Worten:

Satz 1. Charakteristisch für die vom Dreieck $A B C$

um einen Punkt $P$ erzeugte Strahleninvolution $(P)^{2}$ ist, daß in ihr je zwei Strahlen, deren Pole mit $P$ in einer Geraden liegen, einander zugepaart sind und daß die Pole eines solchen Strahlenpaares ihrerseits in der von $A B C$ aufihrer Verbindungsgeraden erzeugten Punkt- auf einer Geraden $p$ erzeugte Punktinvolution $(p)^{2}$ ist, daß in ihr je zwei Punkte, deren Polaren mit $p$ durch einen Punkt gehen, einander zugepaart sind und daß die Polaren eines solchen Punktepaares ihrerseits in der von $A B C$ um ihren Schnittpunkt erzeugten Strahleninvolution zweiten Grades ein Paar bilden. 
Der erste Teil dieses Satzes links ist nur eine Wiederholung des vorhergehenden links und der zweite Teil folgt aus der Umkehrung des vorhergehenden rechts; denn die Verbindungsgerade der Pole der ein Paar in $(P)^{2}$ bildenden Strahlen geht durch den Schnittpunkt $P$ der letzteren und somit schneiden sich die letztere, die Polaren, auf der Verbindungsgeraden ihrer Pole.

Der vorstehende Satz bleibt auch dann richtig, wenn der Punkt $P$ auf einer Seite von $A B C$ liegt bzw. rechts, wenn die Gerade $p$ durch eine Ecke geht. Denn in der von $A B C$ um einen solchen Punkt $P$ erzeugten parabolischen Strahleninvolution ist jedem Strahle jene Seite zugepaart, ferner kann in diesem Falle $P$ als $\mathrm{Pol}$ jener Seite angesehen werden (Nr.1), und endlich muß dann der Pol jeder durch $P$ gehenden Geraden, weil er von $P$, dem Schnittpunkte seiner Polare mit jener Seite von $A B C$, durch die beiden anderen Seiten harmonisch getrennt ist (Nr. 1), zu $P$ zugepaart sein in der von $A B C$ auf der Geraden, die ihn mit $P$ verbindet, erzeugten Punktinvolution zweiten Grades (Nr. 4).

8. Wir wollen nun die durch $P$ gehende Gerade, welche die Pole eines Strahlenpaares von $(P)^{2}$ verbindet, und den auf $p$ liegenden Punkt, in welchem die Polaren eines Punktepaares von $(p)^{2}$ sich schneiden, den Repräsentanten jenes Strahlenpaares bzw. den Repräsentanten dieses Punktepaares nennen.

Nunmehr gilt der

Satz 2. Die von $A B C$ in einem Gebilde erster Stufe erzeugte Involution zweiten Grades ist zum Gebilde der Repräsentanten (welches letztere Gebilde mit dem ersteren konjektiv ist) projektiv, so daß jedem Paare sein Repräsentant entspricht.

Denn eine Involution zweiten Grades wird bekanntlich dadurch projektiv bezogen, daß man die irgendeinem festen Elemente des Trägers der Involution in bezug auf die Paare derselben zugeordneten vierten harmonischen Elemente projektiv bezieht. Nun ist der Büschel erster Ordnung um $P$, welcher von dem einem festen Strahl $g_{i}$ von $P$ in bezug auf ein bewegliches Strahlenpaar $g_{x} g_{x}^{\prime}$ von $(P)^{2}$ zugeordneten vierten harmonischen Strahl $g$ beschrieben wird, wenn das Paar $g_{x} g_{x}^{\prime}$ die Involution $(P)^{2}$ durchläuft, zu der Punktreihe auf dem Polarkegelschnitt $p^{2}$ von $P$, welche 
von dem Pole $G$ von $g$, also von dem auf $p^{2}$ dem festen Pole $G_{i}$ von $g_{i}$ in bezug auf das von den Polen des beweglichen Strahlenpaares gebildete und die induzierte Involution $\left(p^{2}\right)^{2}$ durchlaufende Punktepaar $G_{x} G_{x}^{\prime}$ zugeordneten vierten harmonischen Punkte $G$ beschrieben wird, projektiv. Diese Punktreihe auf $p^{2}$ wird aber von dem auf $p^{2}$ liegenden Pole $G_{i}$ aus durch einen Strahlenbüschel projiziert, welcher zu demjenigen Büschel um $P$ projektiv ist, der von der das bewegliche Punktepaar $G_{x}^{\prime \prime} G_{x}^{\prime}$ verbindenden Geraden beschrieben wird; denn je zwei entsprechende Strahlen in diesen Büscheln müssen, weil sie den Kegelschnitt $p^{2}$ in vier harmonischen Punkten schneiden, in bezug auf $p^{2}$ konjugiert sein. Mithin sind auch der erste und letzte Büschel um $P$, nämlich der von dem zu $g_{i}$ in bezug auf das bewegliche Strahlenpaar $g_{x} g_{x}^{\prime}$ zugeordneten vierten harmonischen $g$ beschriebene und der von der die Pole $G_{x}$ und $G_{x}^{\prime}$ desselben Strahlenpaares $g_{x} g_{x}^{\prime}$ verbindenden Geraden beschriebene projektiv; womit der vorstehende Satz bewiesen ist.

9. Da die Pole eines Strahlenpaares von $(P)^{2}$ mit $P$ in einer Geraden liegen (Satz 1) und die Polaren der auf einer Geraden liegenden Punkte einen dem Dreieck $A B C$ eingeschriebenen Kegelschnitt tangieren (Nr. 2), so folgt:

Zwei Gerade sind nur dann in der von $A B C$ um ihren Schnittpunkt erzeugten Involution zweiten Grades einander zugepaart, wenn sie zusammen mit der Polare ihres Schnittpunktes und den Seiten von $A B C$ sechs Tangenten eines und desselben Kegelschnittes bilden.
Zwei Punkte sind $\mathrm{nur}$ dann in der von $A B C$ auf ihrer Verbindungsgeraden erzeugten Involution zweiten Grades einander zugepaart, wenn sie zusammen mit dem Pole ihrer Verbindungsgeraden und den Ecken von $A B C$ sechs Punkte eines und desselben Kegelschnittes bilden.

10. Die in dem Polarkegelschnitt $p^{2}$ von $P$ (wo $P$ ein auf keiner Seite von $A B C$ liegender Punkt ist) induzierte Punktinvolution $\left(p^{2}\right)^{2}$ wird nun aus jedem Punkte von $p^{2}$ auf ihre Achse, die Polare $p$ von $P(\mathrm{Nr} .7)$, in die von $p^{2}$ und $A B C$ auf $p$ erzeugte Involution $(p)^{2}(\mathrm{Nr} .2)$ projiziert. Mithin wird auch die induzierte Punktinvolution $\left(p^{2}\right)^{3}$ aus jedem Punkte von $p^{2}$ auf $p$ in $(p)^{3}$ pro- 
jiziert (nach Anmerkung in Nr.4). Die Involutionen $\left(p^{2}\right)^{2}$ und $\left(p^{2}\right)^{3}$, welche aus der ternären zyklischen Projektivität

\section{$A B C \pi B C A$}

auf $p^{2}$ hervorgehen, werden aber aus jedem Punkte $G$ von $p^{2}$ durch die von $A B C$ um diesen Punkt erzeugten Strahleninvolutionen $(G)^{2}$ und $(G)^{3}$, welche letztere aus der ternären zyklischen Projektivität

$$
G A, G B, G C \pi G B, G C, G A
$$

hervorgehen, projiziert. Demnach sind die von $A B C$ um einen Punkt von $p^{2}$ erzeugten Strahleninvolutionen zweiten und dritten Grades zu $(p)^{2}$ und $(p)^{3}$ perspektiv.

Soll nun eine und folglich jede der beiden von $A B C$ um einen nicht auf $p^{2}$ liegenden Punkt $P_{1}$ erzeugten Strahleninvolutionen $\left(P_{1}\right)^{2}$ und $\left(P_{1}\right)^{3} \mathrm{zu}(p)^{2}$ bzw. $(p)^{3}$ perspektiv sein, so wird $P_{1}$ auf keiner Seite von $A B C$ liegen können; da sonst seine Strahleninvolution parabolisch wäre und zu den Punktinvolutionen auf $p$ nicht perspektiv sein könnte. $P_{1}$ wird aber auch nicht auf einer der Tangenten von $p^{2}$ in den Eckpunkten $A, B, C$ liegen können. Denn wäre etwa $P_{1} A$ die Tangente von $p^{2}$ in $A$, so müßte $P_{1} A$ durch den Schnittpunkt ( $p a) \equiv P_{a}$ gehen (Nr. 2) und mithin, weil $P_{a}, P_{b}, P_{c}$ in $(p)^{3}$ und $P_{1} A, P_{1} B, P_{1} C$ in der (nach Annahme) zu $(p)^{3}$ perspektiven $\left(P_{1}\right)^{3}$ je ein Tripel bilden und $P_{1}$ auf keiner Seite liegt, $P_{1} B$ durch $P_{b}$ und $P_{1} C$ durch $P_{c}$ und es gingen also die drei Tangenten $A P_{a}, B P_{b}, C P_{c}$ von $p^{2}$ durch den einen Punkt $P_{1}$, was unmöglich ist. Wird nun $G$ der zweite Schnittpunkt von $P_{1} A$ mit $p^{2}$ sein (wo $G$, wie wir eben sahen, von $A, B, C$ verschieden sein wird), so wird, wenn $X, Y, Z$ der Reihe nach die Schnittpunkte von $p$ mit $P_{1} A, P_{1} B, P_{1} C$ sind und mithin (weil $\left(P_{1}\right)^{3}$ zu $(p)^{3}$ perspektiv ist) ein Tripel in $(p)^{3}$ bilden, $G B$ durch $Z$ und $G C$ durch $Y$ gehen. Denn $G$ liegt auf $p^{2}$ und mithin muß, wie wir sahen, $(G)^{3} \mathrm{zu}(p)^{3}$ perspektiv sein; es inzidiert also das Tripel $G A, G B, G C$ von $(G)^{3}$, weil $G A \equiv P_{1} A$ durch $X$ geht, mit dem Tripel $X Y Z$ von $(p)^{3}$ und $G B$ geht durch $Z$ und $G C$ durch $Y$. Demnach gehen im vollständigen Vierecke $P_{1} G B C$ $P_{1} B$ und $G C$ durch $Y, P_{1} C$ und $G B$ durch $Z, P_{1} G$ durch $X$ und $B C$ durch $P_{a}$; folglich ist $P_{a}$ von $X$ durch $Y$ und $Z$ harmonisch getrennt und $P_{a}$ ist also zu $X$ zugepaart in $(p)^{2}$ (Nr. 4); mithin $X \equiv P_{a}^{\prime}$ (wo $P_{a}^{\prime}$ der zu $P_{a}$ zugepaarte Punkt in $(p)^{2}$ ist). Der Punkt 
$P_{1}$ wird also auf $A P_{a}^{\prime}$ liegen müssen, da $P_{a}^{\prime} \equiv X \equiv\left(P_{1} A, p\right)$. In analoger Weise kann man zeigen, daß $P_{1}$ auch auf $B P_{b}^{\prime}$ und $C P_{c}^{\prime}$ liegen wird (wo $P_{b}^{\prime}$ und $P_{c}^{\prime}$ die zu $P_{b}$ und $P_{c}$ zugepaarten Punkte in $(p)^{2}$ sind). Nun ist aber der Schnittpunkt der drei Ecktransversalen $A P_{a}^{\prime}, B P_{b}^{\prime}, C P_{c}^{\prime}$ der Pol $P$ von $p(\mathrm{Nr} .5)$; mithin wird der nicht auf $p^{2}$ liegende Punkt $P_{1}$ kein anderer als der Pol $P$ von $p$ sein.

Wir haben demnach:

Satz 3. Die vom Dreieck $A B C$

um einen Punkt $P$ erzeugten Strahleninvolutionen $(P)^{2}$ und $(P)^{3}$ sind außer zu den von $A B C$ auf der Polare $p$ von $P$ erzeugten Punktinvolutionen $(p)^{2}$ und $(p)^{3}$, auf einer Geraden $p$ erzeugten Punktinvolutionen $(p)^{2}$ und $(p)^{3}$ sind außer zu den von $A B C$ um den Pol $P$ von $p$ erzeugten Strahleninvolutionen $(P)^{2}$ und $(P)^{3}$, $\mathrm{zu}$ welchen sie in der besonderen oben (Nr.5) angegebenen Weise perspektiv sind, nur noch zu den von $A B C$ auf den sämtlichen Tangenten $q$ des Polarkegelschnittes $P^{2}$ von $p$ (Nr. 2) erzeugten Punktinvolutionen $(q)^{2}$ und $(q)^{3}$ perspektiv. um die sämtlichen Punkte $G$ des Polarkegelschnittes $p^{2}$ von $P$ (Nr. 2) erzeugten Strahleninvolutionen $(G)^{2}$ und $(G)^{3}$ perspektiv.

Dieser Satz bleibt auch dann richtig, wenn der Punkt $P$ auf einer Seite von $A B C$ liegt, bzw. wenn rechts die Gerade $p$ durch eine Ecke von $A B C$ geht. Denn liegt $P$ etwa auf der Seite $a \equiv B C$, so ist $a$ die Polare von $P$ und die Tangenten des Polarkegelschnittes von $a$ sind die durch $B$ oder $C$ gehenden Geraden (Nr. 2); alsdann liegen die Doppelpunkte einer jeden der von $A B C$ auf diesen Geraden erzeugten parabolischen Involutionen in $B$ bzw. in $C$ vereinigt (Nr. 6) und die Doppelstrahlen der von $A B C$ um $P$ erzeugten parabolischen Involutionen in $a$; mithin ist die letztere Involution $\mathrm{zu}$ allen ersteren perspektiv.

11. Wir wollen für den Satz 3 noch einen zweiten Beweis geben, der uns zugleich eine Beziehung zwischen Pol und Polare und den zugehörigen Polarkegelschnitten hinsichtlich des durch $A B C$ in Gebilden erster Stufe festgelegten Sinnes (Ende dieser Nummer) erschließen wird. 
Ist $p$ eine durch keine Ecke von $A B C$ gehende Gerade, sind $X Y Z$ und $T U V$ irgend zwei Tripel in $(p)^{3}$ und ist der Sinn $X Y Z$ zum Sinne $T U V$ entgegengesetzt, so werden diese Tripel aus einem Punkte $R$, aus dem $(p)^{2}$ durch eine rechtwinklige Strahleninvolution und $(p)^{3}$ durch eine Involution der regelmäßigen Dreistrahlen projiziert werden (Nr.4), auf drei Arten durch drei Paare je einer symmetrischen Strahleninvolution projiziert und zwar durch:

$$
R(X T, Y U, Z V), \quad R(X U, Y V, Z T), \quad R(X V, Y T, Z U) ;
$$

die Doppelstrahlen dieser drei symmetrischen Involutionen sind nämlich die zueinander rechtwinkligen Halbierungsgeraden der von bzw. $R X$ und $R T, R X$ und $R U, R X$ und $R V$ gebildeten Winkel. Weil nun in jeder dieser drei symmetrischen Strahleninvolutionen um $R$ die (imaginären) Doppelstrahlen der rechtwinkligen Strahleninvolution um $R$, welche Doppelstrahlen die (imaginären) Doppelpunkte von $(p)^{2}$ projizieren, ein Paar bilden, so müssen

\section{$X T, Y U, Z V$ und ebenso $X U, Y V, Z T$ und $X V, Y T, Z U$} drei Punktepaare je einer solchen hyperbolischen Involution auf $p$ sein, in der die (imaginären) Doppelpunkte von $(p)^{2}$ ein Paar bilden, also drei Punktepaare je einer auf $(p)^{2}$ sich stützenden hyperbolischen Involution. Dagegen können die drei Punktepaare

$X T, Y V, Z U$ und ebenso $X U, Y T, Z V$ und $X V, Y U, Z T$ nicht in Involution sein, wenn $T U V$ nicht gerade das zu $X Y Z$ harmonische Tripel ist; ist aber dies der Fall und ist etwa $T$ der zu $X$ zugeordnete vierte harmonische Punkt in bezug auf $Y$ und $Z$, so sind dann die drei Punktepaare $X T, Y V, Z U$ (nicht aber $X U, Y T, Z V$ und $X V, Y U, Z T)$ in Involution, und zwar in der Involution $(p)^{2}$. Denn solche drei Punktepaare werden von $R$ aus durch einen konstanten Winkel projiziert und die beiden Schenkel eines um seinen Scheitel sich drehenden konstanten Winkels bilden nur dann eine Involution, wenn der konstante Winkel ein rechter ist; das letztere tritt aber nur dann ein, wenn das Tripel $T U V$ aus den in $(p)^{2}$ zu $X, Y, Z$ zugepaarten Punkten besteht, wenn also (Nr. 4) das Tripel $T U V$ das zu $X Y Z$ harmonische ist.

Das Nämliche gilt von der zu $(p)^{3}$ perspektiven Involution $(P)^{3}$ (Nr. 5). 
Nun sei $X Y Z$ irgendein Tripel von $(p)^{3}$, dessen Sinn zu dem Sinne des von den drei Schnittpunkten von $p$ mit den Dreieckseiten $a, b, c$ gebildeten Tripels $P_{a} P_{b} P_{c}$ entgegengesetzt ist, so sind, wie wir eben sahen, $P_{a} X, P_{b} Y, P_{c} Z$ drei Punktepaare einer hyperbolischen Involution $(I)$ auf $p$, in der die imaginären Doppelpunkte von $(p)^{2}$, also (Nr. 2) die imaginären Schnittpunkte von $p$ mit dem Polarkegelschnitt $p^{2}$ von $P$, dem Pole von $p$, ein Paar bilden. Folglich muß durch den Schnittpunkt $G$ der beiden Ecktransversalen $A X$ und $B Y$ auch die Ecktransversale $C Z$ gehen und jener Punkt $G$ muß auf dem Polarkegelschnitt $p^{2}$ liegen. Denn das vollständige Viereck $A B C G$, in welchem $B C$ und $A G$ durch $P_{a}$ und $X, C A$ und $B G$ durch $P_{b}$ und $Y$ gehen, schneidet in $p$ die Involution $(I)$ ein und $C G$ muß also, weil $A B$ durch $P_{c}$ geht, durch den zu $P_{c}$ zugepaarten Punkt $Z$ in $(I)$ gehen; ferner muß der Polarkegelschnitt $p^{2}$, der durch $A, B, C$ geht und außerdem noch durch ein Punktepaar in der von $A B C G$ in $p$ eingeschnittenen Involution $(I)$, nämlich durch das von den imaginären Doppelpunkten von $(p)^{2}$ gebildete Paar, auch durch die vierte Ecke $G$ des Vierecks $A B C G$ gehen. Die Ecktransversalen $B Z$ und $C Y$ aber schneiden sich nicht auf $A X$, wenn $X$ mit dem zu $P_{a}$ zugepaarten Punkte $P_{a}^{\prime}$ in $(p)^{2}$ nicht zusammenfällt; denn sonst müßten auch $P_{a} X, P_{b} Z, P_{c} Y$ als Schnittpunkte von $p$ mit den Gegenseitenpaaren des von $A, B, C$ und dem gemeinsamen Schnittpunkte der drei Ecktransversalen $A X, B Z, C Y$ gebildeten Vierecks drei Punktepaare einer Involution bilden, was aber unmöglich ist, da die Sinne $P_{a} P_{b} P_{c}$ und $X Z Y$ miteinander übereinstimmen. Fällt aber $X$ mit $P_{a}^{\prime}$ und mithin (nach Nr. 4) $Y$ mit $P_{c}^{\prime}$ und $Z$ mit $P_{b}^{\prime}$ zusammen, so schneiden sich auf $A X \equiv A P_{a}^{\prime}$ außer $B Y \equiv B P_{c}^{\prime}$ und $C Z \equiv C P_{b}^{\prime}$, welche im zweiten Schnittpunkte von $A P_{a}^{\prime}$ mit $p^{2}$ sich schneiden, noch $B Z \equiv B P_{b}^{\prime}$ und $C Y \equiv C P_{c}^{\prime}$, welche letztere in dem auf $A P_{a}^{\prime}$ liegenden Pole $P$ von $p$ sich schneiden (Nr. 5).

Wenn nun $X$ die ganze Gerade $p$ durchläuft, so liegt auf $A X$ je ein und, solange $X$ mit $P_{a}^{\prime}$ nicht zusammenfällt, nur ein solcher Punkt, nämlich der zweite Schnittpunkt $G$ von $A X$ mit $p^{2}$, von welchem aus durch die drei Ecktransversalen $G A, G B, G C$ ein Tripel in $(p)^{3}$ projiziert wird (welches Tripel kein anderes als das $X$ enthaltende sein kann); kommt aber $X$ nach $P_{a}^{\prime}$, so gibt es auf $A X \equiv A P_{a}^{\prime}$ noch einen zweiten solchen Punkt, nämlich den Pol $P$ von $p$. Die von $A B C$ um einen solchen Punkt und nur um einen 
solchen erzeugten Involutionen sind $\mathrm{zu}(p)^{2}$ bzw. $(p)^{3}$ perspektiv; denn nur dann ist die von der ternären zyklischen Projektivität, welche von $A B C$ um den Punkt erzeugt wird, in $p$ eingeschnittene Projektivität mit der von $A B C$ auf $p$ erzeugten ternären zyklischen Projektivität, welche mit der eingeschnittenen einen Zyklus gemein hat, identisch und mithin sind auch die aus der in $p$ eingeschnittenen Projektivität hervorgehenden Involutionen mit $(p)^{2}$ bzw. $(p)^{3}$ identisch. Hiermit ist der Satz 3 nochmals bewiesen.

Zugleich ergibt sich hieraus, wenn wir den Sinn $x y z$ in einem Büschel erster Ordnung und den Sinn $T U V$ auf einer Geraden $p$ als übereinstimmend oder entgegengesetzt ansehen, je nachdem der Sinn der Spuren $p(x y z)$ mit dem Sinne $T U V$ auf $p$ übereinstimmt oder nicht ${ }^{1}$ ):

Verstehen wir unter dem durch $A B C$ um einen auf keiner Seite desselben liegenden Punkt $P$

auf einer durch keine Ecke desselben gehenden Geraden $p$

festgelegten Sinn denjenigen, welcher durch

$$
p_{A} p_{B} p_{C} \equiv P(A B C) \quad \mid \quad P_{a} P_{b} P_{c} \equiv p(a b c)
$$

bestimmt wird, so stimmen die beiden durch $A B C$ um den Pol $P$ und auf seiner Polare $p$ festgelegten Sinne überein, dagegen sind die beiden durch $A B C$

um $P$ und auf irgendeiner Tangente $q$ des Polarkegelschnitts $P^{2}$ seiner Polare $p$ festgelegten Sinne entgegensetzt. auf $p$ und um irgendeinen Punkt $G$ des Polarkegelschnitts $p^{2}$ ihres Poles $P$ festgelegten Sinne entgegensetzt.

\section{§ 3.}

12. Wie wir sahen (Nr. 10), werden die beiden Involutionen $(p)^{3}$ und $\left(p^{2}\right)^{3}$ von irgendeinem auf $p^{2}$ liegenden Punkte aus ineinander projiziert und, wie aus Nr. 11 hervorgeht, werden die Projektionen der Punkte eines Tripels in $\left(p^{2}\right)^{3}$, dessen Sinn mit dem Sinne $A B C$ auf $p^{2}$ übereinstimmt, von irgendeinem auf $p^{2}$ liegenden Punkte aus auf $p$ ein solches Tripel in $(p)^{3}$ bilden, dessen Sinn zum Sinne $P_{a} P_{b} P_{c}$ entgegengesetzt ist.

1) Siehe v. Staudt, Beiträge zur Geometrie der Lage, Nr. 48, S. 30. 
Sind nun $G_{i} G_{k} G_{l}$ und $G_{q} G_{r} G_{s}$ irgend zwei Tripel in $\left(p^{2}\right)^{3}$, deren Sinne mit dem Sinne $A B C$ auf $p^{2}$ übereinstimmen und werden $G_{i}, G_{k}, G_{l}$ von $G_{q}$ aus auf $p$ der Reihe nach in $X, Y, Z$ projiziert, welche letztere Punkte also ein zu $P_{a} P_{b} P_{c}$ entgegengesetzten Sinn habendes Tripel in $(p)^{3}$ bilden, so muß, weil auch $G_{q}, G_{r}, G_{s}$ von $G_{i}$ aus auf $p$ in ein ebensolches Tripel von $(p)^{3}$ projiziert werden, welches letzte Tripel $X$ enthält und mithin mit $X Y Z$ identisch ist auch dem Sinne nach, $G_{i} G_{r}$ durch $Y$ und $G_{i} G_{s}$ durch $Z$ gehen. Aus demselben Grunde müssen $G_{k} G_{r}$ durch $Z, G_{k} G_{s}$ durch $X, G_{l} G_{r}$ durch $X$ und $G_{l} G_{s}$ durch $Y$ gehen. Es gehen also $G_{i} G_{q}, G_{k} G_{s}, G_{l} G_{r}$ durch $X, G_{i} G_{r}, G_{k} G_{q}$, $G_{l} G_{s}$ durch $Y$ und $G_{i} G_{s}, G_{k} G_{r}, G_{l} G_{q}$ durch $Z$.

Nun müssen in den $p^{2}$ eingeschriebenen vollständigen Vierecken $G_{i} G_{q} G_{k} G_{s}$ und $G_{i} G_{q} G_{l} G_{r}$, deren einer Diagonalpunkt $X$ ist, die je beiden andern Diagonalpunkte auf der Polare von $X$ in bezug auf $p^{2}$ liegen; es ist also die Verbindungsgerade der beiden Schnittpunkte $\left(G_{i} G_{k}, G_{q} G_{s}\right)$ und $\left(G_{i} G_{l}, G_{q} G_{r}\right)$, welche Gerade keine andere als die Achse der beiden perspektiven Dreiecke $G_{i} G_{k} G_{l}$ und $G_{q} G_{s} G_{r}$, die $X$ zum Zentrum haben, ist, die Polare von $X$ in bezug auf $p^{2}$. Weil aber der Pol $P$ von $p$ in bezug auf das Grunddreieck $A B C$ zugleich auch Pol von $p$ in bezug auf die Polarkegelschnitte $x^{2}$ von $P$ und $P^{2}$ von $p$ ist und die Involutionen der in bezug auf $p^{2}$ und $P^{2}$ konjugierten Elemente um $P$ und auf $p$ mit $(P)^{2}$ und $(p)^{2}$ identisch sind (Nr. 2) und also die Polare eines auf $p$ liegenden Punktes durch den in $(p)^{2}$ zu diesem Punkte zugepaarten Punkt und $P$ geht, so muß die Achse der beiden perspektiven, $X$ zum Zentrum habenden Dreiecke $G_{i} G_{k} G_{l}$ und $G_{q} G_{s} G_{r}$, welche Achse zugleich Polare des auf $p$ liegenden Zentrums $X$ in bezug auf $p^{2}$ und mithin auch in bezug auf $P^{2}$ ist, durch $P$ gehen und von $X$ durch $Y$ und $Z$ harmonisch getrennt sein (nach Nr.4). Mithin:

\section{Satz 4. Zwei Dreiecke, deren}

Ecken je ein Tripel in der im Polarkegelschnitt $p^{2}$ von $P$ (wo $P$ ein auf keiner Seite des Grunddreiecks $A B C$ liegender Punkt ist) induzierten Punktinvolution $\left(p^{2}\right)^{3}$
Seiten je ein Tripel in der im Polarkegelschnitt $P^{2}$ von $p$ (wo $p$ eine durch keine Ecke des Grunddreiecks $A B C$ gehende Gerade ist)induzierten Tangenteninvolution $\left(P^{2}\right)^{3}$ 
bilden, liegen auf drei Arten perspektiv; dabei sind in allen drei Arten die Sinne der beiden Tripel, von denen das eine von den

\section{Ecken}

Seiten

des einen Dreiecks gebildet wird und das andere von den homologen

\section{Ecken}

\section{Seiten}

im zweiten Dreieck, auf dem Polarkegelschnitt einander entgegengesetzt. Die drei Perspektivitätszentren dieser beiden Dreiecke bilden ein Tripel in $(p)^{3}$ und die drei Perspektivitätsachsen derselben Dreiecke ein Tripel in $(P)^{3}$ (wo $P$ und $p$ Pol und Polare in bezug auf das Grunddreieck $A B C$ sind); und zwar sind diese beiden Tripel zueinander harmonisch, so daß jedes Zentrum von der entsprechenden Achse durch die beiden andern Zentren harmonisch getrennt ist. Jedes der drei Perspektivitätszentren ist der Pol der entsprechenden Perspektivitätsachse in bezug auf die beiden Polarkegelschnitte $p^{2}$ von $P$ und $P^{2}$ von $p$.

Zusatz. Die beiden im vorstehenden Satze genannten Dreiecke liegen nur dann noch auf eine vierte Art perspektiv, wenn die beiden aus ihren

Ecken gebildeten Tripel in

$$
\left(p^{2}\right)^{3}
$$

Seiten gebildeten Tripel in $\left(P^{2}\right)^{3}$

zueinander harmonisch sind. Alsdann werden $P$ und $p$, die Pol und Polare sind, das vierte Zentrum und die vierte Achse sein, und in der vierten Art werden die Sinne der beiden Tripel, von denen das eine von den Elementen des einen Dreiecks gebildet wird und das andere von den homologen Elementen in dem zweiten Dreieck, auf dem Polarkegelschnitt miteinander übereinstimmen.

Denn jede Perspektivitätsart dieser beiden Dreiecke liefert eine Involution auf $p^{2}$, nämlich diejenige, deren Zentrum und Achse das Zentrum und die Achse der beiden perspektiven Dreiecke sind und in welcher Involution die homologen Ecken in diesen Dreiecken drei Punktepaare bilden. Die Elemente zweier Tripel in $(p)^{3}$ oder in $(P)^{3}$ (Nr.11) und mithin auch die Elemente zweier Tripel in der durch $(p)^{3}$ oder $(P)^{3}$ induzierten $\left(P^{2}\right)^{3}$ bzw. $\left(p^{2}\right)^{3}$ bilden aber nur dann auf vier Arten drei 
Punktepaare je einer Involution, wenn die Tripel zueinander harmonisch sind, und alsdann ist $(p)^{2}$ oder $(P)^{2}$ bzw. $\left(P^{2}\right)^{2}$ oder $\left(p^{2}\right)^{2}$ die Involution der vierten Art. Nun haben $\left(P^{2}\right)^{2}$ und $\left(p^{2}\right)^{2}$ $P$ zum Involutionszentrum und $p$ zur Involutionsachse (Nr.7); ferner müssen zwei Tripel in $\left(p^{2}\right)^{3}$ oder $\left(P^{2}\right)^{3}$, von denen jedes aus den zu den Elementen des zweiten zugepaarten Elementen in der elliptischen Involution $\left(p^{2}\right)^{2}$ bzw. $\left(P^{2}\right)^{2}$ gebildet wird, den nämlichen Sinn auf $p^{2}$ bzw. um $P^{2}$ haben, ebenso wie zwei solche Tripel in $(P)^{3}$ bzw. $(p)^{3}$ den nämlichen Sinn um $P$ bzw. auf $p$ haben (Nr.4).

13. Wie wir sahen (Nr.12), gehen durch den Schnittpunkt $X$ von $p$ mit $\operatorname{der} G_{i}$ und $G_{q}$ verbindenden Geraden (wo $G_{i}$ und $G_{q}$ irgend zwei Punkte des Polarkegelschnitts $p^{2}$ von $P$, dem Pole von $p$, sind) auch die beiden $G_{k}$ mit $G_{s}$ und $G_{l}$ mit $G_{r}$ verbindenden Geraden (wobei $G_{i} G_{k} G_{l}$ und $G_{q} G_{r} G_{s}$ zwei Tripel in $\left(p^{2}\right)^{3}$ bilden und der Sinn des erstern mit dem letztern übereinstimmt); mithin müssen die drei Geraden, welche irgendeinen auf $p$ liegenden Punkt mit den Punkten irgendeines Tripels von $\left(p^{2}\right)^{3}$ verbinden, von $p^{2}$ auch zum zweitenmal in den Punkten eines Tripels von $\left(p^{2}\right)^{3}$ geschnitten werden. Also:

Satz 5. Liegt ein Dreieck, welches

$p^{2}$ eingeschrieben ist, $\mathrm{zu}$

einem zweiten Dreieck, des-

sen Ecken ein Tripel in $\left(p^{2}\right)^{3}$
$P^{2}$ umschrieben ist, zu einem zweiten Dreieck, dessen Seiten ein Tripel in $\left(P^{2}\right)^{3}$

bilden, perspektiv und liegt das Zentrum dieser perspektiven Dreiecke auf $p$ oder geht die Achse derselben durch $P$, so bilden auch die

Ecken des erstern Dreiecks ein Tripel in $\left(p^{2}\right)^{3}$.

Seiten des erstern Dreiecks ein Tripel in $\left(P^{2}\right)^{3}$.

Die Richtigkeit hiervon im Falle, daß links die Perspektivitätsachse durch $P$ geht, ergibt sich aus der Bemerkung, daß dann das Perspektivitätszentrum, welches, wie wir sahen (Nr.12), der Pol der Perspektivitätsachse in bezug auf $p^{2}$ ist, auf der in bezug auf $p^{2}$ und das Grunddreieck $A B C$ gemeinsamen Polare $p$ von $P$ (Nr.2) liegt.

14. Nach Satz 4 liegt das von irgendeinem Tripel in $\left(p^{2}\right)^{3}$ gebildete Dreieck $G_{i} G_{k} G_{l}$ zum Grunddreieck $A B C$, dessen Ecken 
ebenfalls ein Tripel in $\left(p^{2}\right)^{3}$ bilden, auf drei Arten perspektiv und die drei Perspektivitätsachsen bilden ein Tripel in $(P)^{3}$. Diese drei Achsen gehen aber durch die drei Schnittpunkte einer jeden der Seiten von $G_{i} G_{k} G_{l}$ mit den Seiten von $A B C$, welche Schnittpunkte ein Tripel in der von $A B C$ auf jener Seite von $G_{i} G_{k} G_{l}$ erzeugten Involution dritten Grades bilden. Mithin sind die von $A B C$ auf den Seiten von $G_{i} G_{k} G_{l}$ erzeugten Involutionen dritten Grades zu $(P)^{3}$ perspektiv, und diese Seiten von $G_{i} G_{k} G_{l}$, welche von der ganz außerhalb $p^{2}$ liegenden Polare $p$ von $P$ verschieden sind, müssen also (nach Satz 3) Tangenten am Polarkegelschnitt $P^{2}$ von $p$ sein und folglich (nach Satz 5) ein Tripel in der Tangenteninvolution $\left(P^{2}\right)^{3}$ bilden; da das nunmehr $P^{2}$ umschriebene Dreieck $G_{i} G_{k} G_{l}$ zu dem Grunddreieck $A B C$, dessen Seiten ein Tripel in $\left(P^{2}\right)^{3}$ bilden, in der Weise perspektiv liegt, daß die Achse durch $P$ geht. Also:

Satz 6. Bilden die Ecken eines Dreiecks ein Tripel in der im Polarkegelschnitt $p^{2}$ von $P$ induzierten Punktinvolution $\left(p^{2}\right)^{3}$, so bilden die Seiten desselben Dreiecks ein Tripel in der im Polarkegelschnitt $P^{2}$ der Polare $p$ von $P$ induzierten Tangenteninvolution $\left(P^{2}\right)^{3}$; und umgekehrt.

Die Umkehrung des vorstehenden Satzes steht dem direkten dual gegenüber.

Hieraus folgt weiter:

Satz 7. Ist ein Dreieck $p^{2}$ einge- und zugleich $P^{2}$ umschrieben, so bilden seine Ecken und Seiten je ein Tripel in $\left(p^{2}\right)^{3}$ bzw. $\left(F^{2}\right)^{3}$.

Denn ist etwa $G_{i}$ einer seiner Ecken, so bildet das Tripel $G_{i} G_{k} G_{l}$ von $\left(p^{2}\right)^{3}$, wie wir eben sahen, ein $P^{2}$ umschriebenes Dreieck, welches mit dem gegebenen identisch sein muß, da durch $G_{i}$ nur zwei Tangenten an $P^{2}$ gehen.

15. Weil die Geraden, die einen auf $p$ liegenden Punkt mit den Punkten eines Tripels in $\left(p^{2}\right)^{3}$ verbinden, von $p^{2}$ zum zweitenmal ebenfalls in den Punkten eines Tripels von $\left(p^{2}\right)^{3}$ geschnitten werden (Nr.13), so muß die zwei Punkte eines Tripels in $\left(p^{2}\right)^{3}$ verbindende Gerade durch den Schnittpunkt von $p$ mit der Tangente an $\nu^{2}$ im dritten Punkte des nämlichen Tripels gehen. Dual muß die $P$ mit dem Schnittpunkte zweier Tangenten eines 
Tripels von $\left(P^{2}\right)^{3}$ verbindende Gerade durch den Berührungspunkt der dritten Tangente des nämlichen Tripels gehen.

Die drei Punkte, in denen die Seiten eines von einem Tripel in $\left(p^{2}\right)^{3}$ gebildeten Dreiecks $G_{i}\left(G_{k} G_{l}\right.$ von den Tangenten an $p^{2}$ in den gegenüberliegenden Eckpunkten geschnitten werden, liegen also auf $p$, und ihre drei Polaren in bezug auf $p^{2}$, welche bzw. von den drei Punkten durch je zwei Eckpunkte von $G_{i} G_{k} G_{l}$ harmonisch getrennt sind, müssen durch je den dritten Eckpunkt und durch $P$, den Pol von $p$ auch in bezug auf $p^{2}$, gehen; mithin sind $P$ und $p$ Pol und Polare auch in bezug auf das Dreieck $G_{i} G_{k} G_{l}$. Der Polarkegelschnitt von $P$ in bezug auf das Dreieck $G_{i} G_{k} G_{l}$, welcher Kegelschnitt diesem Dreieck umschrieben sein muß und dessen Tangenten in den Eckpunkten desselben Dreiecks von den gegenüherliegenden Seiten in den drei auf $p$, der Polare von $P$ auch in bezug auf dasselbe Dreieck, liegenden Punkten geschnitten werden müssen (Nr.2), ist mit $p^{2}$ identisch. Dual muß der Polarkegelschnitt von $p$ in bezug auf das Dreieck $G_{i} G_{k} G_{l}$, dessen Seiten ein Tripel in $\left(P^{2}\right)^{3}$ bilden, mit $P^{2}$ identisch sein. Die vom Dreieck $G_{i} G_{k} G_{l}$ um $P$ und auf $p$ erzeugten Involutionen zweiten Grades sind nun mit den Involutionen der in bezug auf $p^{2}$, den Polarkegelschnitt von $P$ auch in bezug auf das Dreieck $G_{i} G_{k} G_{l}$, konjugierten Elemente und also mit den vom Grunddreieck $A B C$ um $P$ und auf $p$ erzeugten $(P)^{2}$ und $(p)^{2}$ identisch (nach Nr.2), und mithin sind auch die von $G_{i} G_{k} G_{l}$ um $P$ und auf $p$ erzeugten Involutionen dritten Grades mit den von $A B C$ um $P$ und auf $p$ erzeugten $(P)^{3}$ und $(p)^{3}$ identisch (Anmerkung in Nr.4). Nunmehr müssen die von $G_{i} G_{k} G_{l}$ um die sämtlichen auf $p^{2}$, dem Polarkegelschnitt von $P$ auch in bezug auf $G_{i} G_{k} G_{i}$, liegenden Punkte und auf den sämtlichen Tangenten an $P^{2}$, dem Polarkegelschnitt von $p$ auch in bezug auf $G_{i} G_{k} G_{l}$, erzeugten Involutionen zweiten und dritten Grades, welche zu $(p)^{2}$ und $(p)^{3}$ bzw. zu $(P)^{2}$ und $(P)^{3}$ perspektiv sind (Satz 3), mit den vom Grunddreieck $A B C$ erzeugten identisch sein. Ferner müssen die in bezug auf $G_{i} G_{k} G_{l}$ in $p^{2}$ und $P^{2}$ induzierten Involutionen dritten Grades, welche ebenso wie $\left(p^{2}\right)^{3}$ und $\left(P^{2}\right)^{3}$ von den Zyklen je einer ternären zyklischen Projektivität gebildet werden, mit $\left(p^{2}\right)^{3}$ und $\left(P^{2}\right)^{3}$ identisch sein, da sie mit diesen je ein Tripel gemein haben, nämlich das von den Ecken bzw. Seiten von $G_{i} G_{k} G_{l}$ gebildete; mithin müssen auch 
die in bezug auf $G_{i} G_{k} G_{l}$ in $p^{2}$ und $P^{2}$ induzierten Involutionen zweiten Grades mit $\left(p^{2}\right)^{2}$ und $\left(P^{2}\right)^{2}$ identisch sein. Wir haben also:

Satz 8. In bezug auf jedes Dreieck, dessen Ecken und Seiten je ein Tripel in $\left(p^{2}\right)^{3}$ bzw. in $\left(P^{2}\right)^{3}$ bilden, sind $P$ und $p$ Pol und Polare, $p^{2}$ und $P^{2}$ die Polarkegelschnitte von $P$ und $p$. Die um $P$ und um jeden Punkt von $p^{2}$, auf $p$ und auf jeder Tangente von $P^{2}$ von einem solchen Dreieck erzeugten Involutionen zweiten und dritten Grades sind mit den vom Grunddreieck $A B C$ erzeugten identisch; und die in bezug auf ein solches Dreieck in $p^{2}$ und $P^{2}$ induzierten Involutionen zweiten und dritten Grades sind mit den in bezug auf $A B C$ induzierten $\left(p^{2}\right)^{2}$ und $\left(p^{2}\right)^{3}$ bzw. $\left(P^{2}\right)^{2}$ und $\left(P^{2}\right)^{3}$ identisch.

16. Eine Umkehrung des vorstehenden Satzes ist im folgenden Satze enthalten.

Satz 9. Die Ecken und Seiten eines Dreiecks, in bezug auf welches der Polarkegelschnitt

$$
\text { von } P p^{2} \text { ist, } \quad \text { von } p P^{2} \text { ist, }
$$

bilden je ein Tripel in $\left(p^{2}\right)^{3}$ bzw. in $\left(P^{2}\right)^{3}$. Dasselbe gilt von den Ecken und Seiten eines Dreiecks, welches

$p^{2}$ eingeschrieben ist und $\mid P^{2}$ umschrieben ist und von welchem auf $p$ eine von welchem um $P$ eine mit $(p)^{2}$ mit $(P)^{2}$

identische Involution erzeugt wird, oder welches

$$
p^{2} \text { eingeschrieben } \quad P^{2} \text { umschrieben }
$$

ist und in bezug auf welches $P$ und $p$ Pol und Polare sind.

Beweis. Die drei Verbindungsgeraden von $P$ mit den Ecken eines Dreiecks, welches um $P$ und auf $p$ dieselben Involutionen wie das Grunddreieck $A B C$ erzeugt und in bezug auf welches $P$ und $p$ Pol und Polare sind, und die drei Seiten desselben Dreiecks werden von $p$ in zwei zueinander harmonischen Tripeln von $(p)^{3}$ geschnitten; und zwar geht jede Seite durch denjenigen Punkt von $p$, welcher dem Schnittpunkte von $p$ mit der Geraden, welche letzte die Gegenecke mit $P$ verbindet, zugepaart ist in $(p)^{2}$ (nach Nr. 5). Wenn also nur eine Ecke oder nur eine Seite eines solchen Dreiecks gegeben ist, so ist dadurch das ganze 
Dreieck eindeutig bestimmt. Denn die Verbindungsgerade von $P$ mit jener Ecke, sie heiße etwa $R$, liefert (durch ihren Schnittpunkt $X^{\prime}$ mit $p$ ) die beiden zueinander harmonischen Tripel $X^{\prime} Y^{\prime} Z^{\prime}$ und $X Y Z$ in $(p)^{3}$, wo $X X^{\prime}, Y Y^{\prime}, Z Z^{\prime}$ drei Punktpaare in $(p)^{2}$ sind; die beiden durch jene Ecke $R$ gehenden Seiten des Dreiecks werden $p$ in $Y$ und $Z$ treffen, die beiden anderen Ecken des Dreiecks sind die Schnittpunkte von $R Y$ mit $P Z^{\prime}$ und von $R Z$ mit $P Y^{\prime 1}$ ). Ist also ein solches Dreieck $p^{2}$ eingeschrieben und etwa $G_{i}$ eine seiner Ecken, so muß dieses Dreieck von dem Tripel $G_{i} G_{k} G_{l}$ in $\left(p^{2}\right)^{3}$ gebildet sein; denn es gibt nur ein einziges solches Dreieck, welches $G_{i}$ zu einer seiner Ecken hat, und $G_{i} G_{k} G_{l}$ ist ein solches (Satz 8).

Nunmehr muß ein Dreieck, in bezug auf welches $p^{2}$ der Polarkegelschnitt von $P$ ist, ein solches, $p^{2}$ eingeschriebenes und also von einem Tripel in $\left(p^{2}\right)^{3}$ gebildetes sein. In der Tat muß das Dreieck, in bezug auf welches $p^{2}$ der Polarkegelschnitt von $P$ ist, $p^{2}$ eingeschrieben sein, und die Polare von $P$ in bezug auf dasselbe Dreieck muß dieselbe wie in bezug auf $p^{2}$, also $p$ sein, und die von demselben Dreieck um $P$ und auf $p$ erzeugten Involutionen zweiten und dritten Grades müssen, weil die des zweiten Grades mit den Involutionen der in bezug auf $p^{2}$ konjugierten Elemente identisch sind, mit $(P)^{2}$ und $(P)^{3}$ bzw. $(p)^{2}$ und $(p)^{3}$ identisch sein (Nr. 2).

Ferner muß der Polarkegelschnitt von $P$ in bezug auf ein Dreieck, welches $p^{2}$ eingeschrieben ist und welches auf $p$ eine mit $(p)^{2}$ identische Involution erzeugt, oder welches $p^{2}$ eingeschrieben ist und in bezug auf welches $P$ und $p$ Pol und Polare sind, mit $p^{2}$ identisch sein; denn jener Polarkegelschnitt hat mit $p^{2}$ die Eckpunkte des Dreiecks gemein und außerdem noch die beiden Doppelpunkte von $(p)^{2}$ bzw. $P$ und $p$ zu Pol und Polare (nach Nr. 2). Es müssen also auch solche Dreiecke, wie die letzt erwähnten, von je einem Tripel in $\left(p^{2}\right)^{3}$ gebildet sein.

17. Weil die drei Verbindungsgeraden eines Punktes mit den Ecken eines Dreiecks ein Tripel in der von diesem Dreieck um jenen Punkt erzeugten Involution dritten Grades bilden, so folgt aus Satz 8:

1) Siehe meine Dissertation, IV. Abschnitt, Nr. 16. 
Satz 10. Jedes Punktetripel in $\left(p^{2}\right)^{3}$ wird von $P$ aus durch drei ein Tripel in $(P)^{3}$ bildende Strahlen projiziert.

Umgekehrt, liegen auf jedem Strahlentripel in $(P)^{3}$ sechs Punkte von $p^{2}$, die zwei zueinander harmonische Tripel in $\left(p^{2}\right)^{3}$ und zugleich drei ebenso wie $p_{A} p_{A}^{\prime}, p_{B}^{\prime} p_{B}^{\prime}, p_{C} p_{C}^{\prime}$ harmonich beschaffene Paare in $\left(p^{2}\right)^{2}$ bilden.
Jedes Tangenten-Tripel in $\left(P^{2}\right)^{3}$ wird von $p$ in drei ein Tripel in $(p)^{3}$ bildenden Punkten geschnitten.

Umgekehrt, gehen durch jedes Punktetripel in $(p)^{3}$ sechs Tangenten an $P^{2}$, die zwei zueinander harmonische Tripel in $\left(P^{2}\right)^{3}$, und zugleich drei ebenso wie $P_{a} P_{a}^{\prime}, P_{b} P_{b}^{\prime}, P_{c} P_{c}^{\prime}$ harmonisch beschaffene Paare in $\left(P^{2}\right)^{2}$ bilden.

Die Umkehrung folgt aus dem direkten Satze und Nr. 4, wenn man bemerkt, daß $P$ das Zentrum der durch $(P)^{2}$ in $p^{2}$ induzierten Punktinvolution $\left(p^{2}\right)^{2}$ ist (Nr. 7).

18. Weil die Pole jedes Strahlentripels von $(P)^{3}$ ein Tripel in der durch $(P)^{3}$ in $p^{2}$ induzierten Punktinvolution $\left(p^{2}\right)^{3}$ bilden, so ergibt sich aus den Sätzen 8 und 9:

Satz 11. Charakteristisch für die vom Dreieck $A B C$ um einen Punkt $P$ (wo $P$ auf keiner Seite von $A B C$ liegt) erzeugte Strahleninvolution $(P)^{3}$ ist, daß in ihr die Tripel aus je drei solchen Strahlen bestehen, deren Pole auf einer Geraden $p$ (wo $p$ durch keine Ecke von $A B C$ geht) erzeugte Punktinvolution $(p)^{3}$ ist, daß in ihr die Tripel aus je drei solchen Punkten bestehen, deren Polaren

ein Dreieck bilden, in bezug auf welches Dreieck ebenso wie in bezug auf das Grunddreieck $A B C$
$p$ die Polare von $P$
$P$ der Pol von $p$

ist und $p^{2}$ und $P^{2}$ die Polarkegelschnitte von $P$ und $p$ sind und welches Dreieck um $P$ und auf $p$ dieselben Involutionen wie das Grunddreieck $A B C$ erzeugt.

\section{§4.}

19. Wie wir sahen (Satz 1 in Nr. 7), liegen die beiden Pole eines jeden Strahlenpaares der von $A B C$ um einen Punkt er- 
zeugten Involution zweiten Grades auf einer durch diesen Punkt gehenden Geraden, und umgekehrt. Ermittelt man daher zu jedem Strahle von $P$ (wo $P$ ein auf keiner Seite von $A B C$ liegender Punkt ist) die Polaren der Schnittpunkte des Strahles mit dem Polarkegelschnitt $p^{2}$ von $P$, ermittelt man also dasjenige Strahlenpaar von $P$, dessen Pole von $P$ aus durch jenen Strahl projiziert werden, so bilden diese Strahlenpaare die von $A B C$ um $P$ erzeugte Involution $(P)^{2}$. Ermittelt man nun zu jedem Strahlenpaare von $(P)^{2}$ dasjenige Strahlenquadrupel von $P$, dessen Pole von $P$ aus durch jenes Strahlenpaar projiziert werden, so bilden diese Strahlenquadrupel ein System von $\infty^{1}$ Quadrupel um $P$, welches System wir mit $(P)^{4}$ bezeichnen wollen. Ermittelt man ferner zu jedem Quadrupel von $(P)^{4}$ diejenige Gruppe von acht Strahlen von $P$, deren Pole von $P$ aus durch jenes Quadrupel projiziert werden, so bilden diese Gruppen ein System von $\infty^{1}$ Gruppen von je acht Strahlen um $P$, welches System wir mit $(P)^{8}$ bezeichnen. Indem dieser Prozeß $n$ Mal wiederholt wird, gelangt man zu einem Systeme von $\infty^{1}$ Gruppen von je $2^{n}$ Strahlen um $P$, welches System wir mit $(P)^{2^{n}}$ bezeichnen.

Hiernach führt jeder Strahl von $P$ durch $n$-malige Wiederholung des angegebenen Prozesses zu einer einzigen Gruppe im Systeme $(P)^{2^{n}}$; jenen Strahl wollen wir den Repräsentanten dieser Gruppe nennen. Ebenso führt jede Gruppe im Systeme $(P)^{2^{k}}$ $(k<n)$ durch $(n-k)$-malige Wiederholung desselben Prozesses auf jeden ihrer Strahlen zu einer einzigen Gruppe im Systeme $(P)^{2^{n}}$; die erstere Gruppe nennen wir die Repräsentationsgruppe in $(P)^{2^{k}}$ von der letztern in $(P)^{2^{n}}$. Eine Gruppe in $(P)^{2^{n}}$ nebst allen ihren Repräsentationsgruppen in $(P)^{2^{n-1}},(P)^{2^{n-2}}$ bis $(P)^{2^{n-k}}$ haben eine und dieselbe Repräsentationsgruppe in $(P)^{2^{n-l}}$, wo $k<l \leqq n$ (ist $l=n$, so ist $(P)^{2^{n-l}}=(P)^{2^{0}}$ der einfache Büschel um $P$ und eine Gruppe in $(P)^{1}$ reduziert sich auf einen einzigen Strahl, und die Repräsentationsgruppe in $(P)^{1}$ einer Gruppe von $(P)^{2^{n}}$ ist also der Repräsentant der letzteren Gruppe).

Weil jede Gruppe im Systeme $(P)^{2^{n}}$ auch dadurch entstanden gedacht werden kann, daß man, anstatt von ihrem Repräsentanten auszugehen und den angegebenen Proze $n n$ Mal zu wiederholen, von den $2^{k} \quad(k<n)$ ihre Repräsentationsgruppe in $(P)^{2^{k}}$ bildenden Strahlen ausgeht und den angegebenen Prozeß nur $n-k \mathrm{Mal}$ wiederholt, so muß eine Gruppe von $(P)^{2^{n}} 2^{k}$ Gruppen des Systems 
$(P)^{2^{n-k}}$ enthalten, also: $2^{n}$ Strahlen von $P, 2^{n-1}$ Strahlenpaare von $(P)^{2}, 2^{n-2}$ Strahlenquadrupel von $(P)^{4}$ usw.

Die Repräsentationsgruppe in $(P)^{2^{k}}$ einer Gruppe von $(P)^{2^{n}}$ besteht demnach aus den Repräsentanten der $2^{k}$ in der letzteren Gruppe enthaltenen Gruppen von $(P)^{2^{n-k}}$.

Um zu einer Gruppe von $(P)^{2^{n}}$ ihren Repräsentanten aufzufinden, haben wir $P$ mit dem Pole irgendeines Strahles der Gruppe durch eine Gerade zu verbinden, darauf mit dem Pole dieser Verbindungsgeraden durch eine zweite Gerade, sodann mit dem Pole der zweiten Verbindungsgeraden durch eine dritte und wiederholen diese Operation $n \mathrm{Mal}$; die $n$-te hierdurch erhaltene Verbindungsgerade ist der gesuchte Repräsentant. Es ist also durch einen Strahl die ganze Gruppe, der er angehört, im Systeme $(P)^{2^{n}}$ eindeutig bestimmt; wir ermitteln nämlich zuerst den Repräsentanten der Gruppe, sodann, von diesem Repräsentanten ausgehend, die ganze Gruppe.

Jeder Strahl von $P$ gehört also einer einzigen Gruppe im Systeme $(P)^{2^{n}}$ an.

Duales gilt für jede durch keine Ecke von $A B C$ gehende Gerade.

20. Ermittelt man ebenso zu jedem Tripel in der von $A B C$ um $P$ erzeugten Strahleninvolution $(P)^{3}$ diejenige Gruppe von sechs Strahlen von $P$, deren Pole von $P$ aus durch jenes Tripel projiziert werden, so bilden diese Gruppen ein System $(P)^{6}$ von $\infty^{1}$ Gruppen von je sechs Strahlen; jede Gruppe dieses Systems besteht aus drei ebenso wie $p_{A} p_{A}^{\prime}, p_{B} p_{B}^{\prime}, p_{C} p_{C}^{\prime}$ harmonisch beschaffenen Paaren von $(P)^{2}$ und zugleich aus zwei zueinander harmonischen Tripeln von $(P)^{3}$ (nach Satz 10 in Nr. 17). Ermittelt man nun $\mathrm{zu}$ jeder der Gruppen von $(P)^{6}$ diejenige Gruppe von zwölf Strahlen von $P$, deren Pole von $P$ aus durch die erstere Gruppe projiziert werden, so bilden die letzteren Gruppen ein System $(P)^{12}$ von $\infty^{1}$ Gruppen von je zwölf Strahlen. Durch $n$-malige Wiederholung dieses Prozesses gelangt man zu einem Systeme $(P)^{2^{n} \cdot 3}$ von $\infty^{1}$ Gruppen von je $2^{n} .3$ Strahlen; jede Gruppe dieses Systems enthält $2^{n-1} \cdot 3$ Srahlenpaare von $(P)^{2}$, $2^{n}$ Tripel von $(P)^{3}$ und überhaupt $2^{k} \cdot 3$ und $2^{k}$ Gruppen von $(P)^{2^{n-k}}$ bzw. $(P)^{2^{n-k}} \cdot 3$, wo $0 \leqq k \leqq n$. Jedes Tripel von $(P)^{3}$ führt $\mathrm{zu}$ einer einzigen Gruppe in $(P)^{2^{n} \cdot 3}$; jenes Tripel nennen wir das Repräsentationstripel dieser Gruppe. 
Weil jede Gruppe in $(P)^{2^{n} \cdot 3}$ aus denjenigen drei Gruppen von $(P)^{2^{n}}$ besteht, deren Repräsentanten das Repräsentationstripel der ersteren Gruppe bilden, so wird das Repräsentationstripel einer Gruppe von $(P)^{2^{n} \cdot 3}$, wenn auch nur ein Strahl dieser Gruppe gegeben ist, dadurch bestimmt, daß man den Repräsentanten derjenigen Gruppe von $(P)^{2^{n}}$, der der gegebene Strahl angehört, aufsucht, sodann das diesen Repräsentanten enthaltende Tripel in $(Y)^{3}$ bestimmt; dieses letztere wird das gesuchte Repräsentationstripel sein. Es gehört also jeder Strahl von $P$ einer einzigen Gruppe in $(P)^{2^{n} \cdot 3}$ an.

Duales gilt für jede durch keine Ecke von $A B C$ gehende Gerade.

21. Ich behaupte nun:

Die auf diese Weise entstehenden Systeme $(P)^{2^{n}}$ und $(P)^{2^{n} \cdot 3}$ sind (für jeden positiven ganzen Wert von $n$ ) zy klische Projektivitäten $\left(2^{n}\right)$ - bzw. $\left(2^{n} .3\right)$-ten Grades erster Art; die Gruppen in jenen bilden die Zyklen in diesen. Die Koinzidenzstrahlen dieser Projektivitäten sind die (konjugiertimaginären) Doppelstrahlen von $(P)^{2}$.

Die Richtigkeit dieser Behauptung kann durch den Schluß von $n$ auf $n+1$ bewiesen werden.

Denn ist irgendeine zyklisch-projektive Gruppe $k$-ten Grades gegeben:

$$
A_{1} A_{2} A_{3} \ldots A_{k} \pi A_{2} A_{3} A_{4} \ldots A_{1}
$$

so bilden bekanntlich ${ }^{1}$ ) die aus diesen $k$ Elementen zusammengesetzten Paare, deren Zeigersumme zu einer und derselben Zahl kongruent ist (mod. $k$ ), eine Involution, in der die Koinzidenzelemente der zyklischen Projektivität ein Paar bilden. Und bilden, umgekehrt, die aus den $k$ Elementen $A_{1}, A_{2}, A_{3}, \ldots A_{k}$ zusammengesetzten Paare, deren Zeigersumme etwa $\equiv 1(\bmod . k)$ ist, eine Involution:

$$
A_{1} A_{k}, A_{2} A_{k-1}, A_{3} A_{k-2}, \ldots
$$

und die Paare, deren Zeigersumme $\equiv 2(\bmod k)$ ist, eine zweite Involution:

$$
A_{1} A_{1}, A_{2} A_{k}, A_{3} A_{k-1}, A_{4} A_{k-2}, \ldots,
$$

so muß wegen der ersten Involution:

und wegen der zweiten:

$$
A_{1} A_{2} A_{3} \ldots A_{k} \pi A_{k} A_{k-1} A_{k-2} \ldots A_{1}
$$

$$
A_{k} A_{k-1} A_{k-2} \ldots A_{1} \pi A_{2} A_{3} A_{4} \ldots A_{1}
$$

sein, folglich: $\quad A_{1} A_{2} A_{3} \ldots A_{k} \pi A_{2} A_{3} A_{4} \ldots A_{1}$,

1) Vgl. Sturm, Geometrische Verwandschaften I, Nr.139. 
also bilden dann die $k$ Elemente eine zyklisch-projektive Gruppe und das gemeinsame Paar jener beiden Involutionen liefert die Koinzidenzelemente dieser Projektivität.

Ferner ist charakteristisch für eine zyklisch-projektive Gruppe $k$-ten Grades erster Art:

$$
A_{1} A_{2} A_{3} \ldots A_{k} \pi A_{2} A_{3} A_{4} \ldots A_{1},
$$

wo also das Gebilde von einem Zyklus nur einmal durchlaufen wird, daß die Elemente $A_{1}, A_{2}, A_{3}, \ldots A_{k}$ in der natürlichen Anordnung in einem der beiden Sinne im Gebilde aufeinander

Fig. 2.

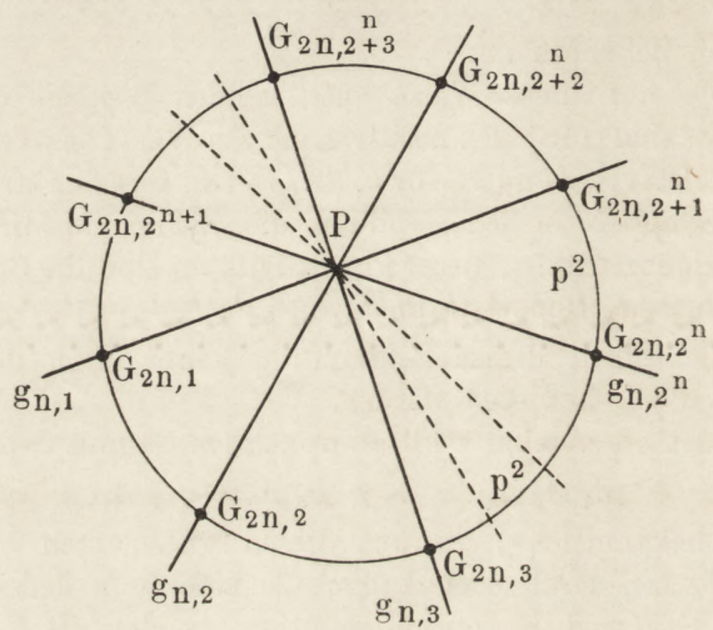

folgen, so daß zwischen zwei aufeinanderfolgenden Elementen des Zyklus kein anderes Element desselben Zyklus liegt.

Ist nun unsere Behauptung für einen bestimmten Wert von $n$ richtig, bilden also für diesen Wert von $n$ die $2^{n}$ Strahlen einer Gruppe von $(P)^{2^{n}}$ eine zyklisch-projektive Gruppe $\left(2^{n}\right)$-ten Grades erster Art:

$$
\text { П) } g_{n, 1} g_{n, 2} g_{n, 3} \ldots g_{n, 2^{n}} \pi g_{n, 2} g_{n, 3} g_{n, 4} \ldots g_{n, 1} \text {, }
$$

wo $g_{n, 1} g_{n, 2} g_{n, 3} \ldots g_{n, 2^{n}}$ in der natürlichen Anordnung in einem der beiden Sinne im Büschel um $P$ aufeinander folgen und wo die Koinzidenzstrahlen von $\Pi$ die Doppelstrahlen von $(P)^{2}$ sind, und schneiden (Fig. 2)

$$
g_{n, 1}, g_{n, 2}, g_{n, 3}, \ldots g_{n, 2^{n}}
$$


den Polarkegelschnitt $p^{2}$ von $P$ der Reihe nach in den Punktepaaren

$G_{2 n, 1} G_{2 n, 2^{n}+1}, G_{2 n, 2} G_{2 n, 2^{n}+2}, G_{2 n, 3} G_{2 n, 2^{n}+3}, \ldots G_{2 n, 2^{n}} G_{2 n, 2^{n+1}}$, wo die auf der einen Seite des Strahles $g_{n, 1}$, und zwar auf derjenigen, wo $g_{n, 2}$ gleich auf dem Halbstrahl $P G_{2 n, 1}$ (mit $G_{2 n, 1}$ soll beliebig einer der beiden Schnittpunkte von $g_{n, 1}$ mit $p^{2}$ bezeichnet werden) folgt, liegenden Schnittpunkte mit $G_{2 n, 2}, G_{2 n, 3}, \ldots G_{2 n, 2^{n}}$ bezeichnet sind und die auf der zweiten Seite mit $G_{2 n, 2^{n}+2}$, $G_{2 n, 2^{n}+3}, \ldots G_{2 n, 2^{n+1}}$, so folgen, weil $P$ innerhalb seines Polarkegelschnitts $p^{2}$ liegt (Nr. 2),

$G_{2 n, 1} G_{2 n, 2} G_{2 n, 3} \ldots G_{2 n, 2^{n}} G_{2 n, 2^{n}+1} G_{2 n, 2^{n}+2} G_{2 n, 2^{n}+3} \ldots G_{2 n, 2^{n+1}}$ in der natürlichen Anordnung in einem der beiden Sinne in $p^{2}$ aufeinander.

Nunmehr bilden die Strahlenpaare

$$
g_{n, 1} g_{n, 2^{n}}, g_{n, 2} g_{n, 2^{n}-1}, g_{n, 3} g_{n, 2^{n}-2}, \ldots,
$$

deren Zeigersumme $\equiv 1\left(\bmod .2^{n}\right)$ ist, eine Involution $\left(I_{1}\right)$, in der die Koinzidenzstrahlen von $\Pi-$ die, nach der für $n$ geltenden Behauptung, die (konjugiert-imaginären) Doppelstrahlen von $(P)^{2}$ sind - ein Paar bilden; mithin müssen die (reellen) Doppelstrahlen von $\left(I_{1}\right)$ ein Paar in $(P)^{2}$ bilden und also in bezug auf $p^{2}$ einander konjugiert sein (nach Nr. 2). Der Schnittpunkt des einen Doppelstrahles von $\left(I_{1}\right)$ mit der Polare $p$ von $P$ (auch in bezug auf $p^{2}$ ) muß somit der Pol des zweiten Doppelstrahles in bezug auf $p^{2}$ sein. Folglich muß $p^{2}$ von der Geraden $l$, welche $G_{2 n, 1}$ mit dem Pole des innerhalb des Winkels $G_{2 n, 1} P G_{2 n, 2^{n+1}}$ liegenden Doppelstrahles von $\left(I_{1}\right)$ in bezug auf $p^{2}$ verbindet, also mit dem Schnittpunkte von $p$ mit dem innerhalb des Winkels $G_{2 n, 1} P G_{2 n, 2^{n}}$ liegenden Doppelstrahle von $\left(I_{1}\right)$, zum zweitenmal in einem solchen Punkte geschnitten werden, welcher von $G_{2 n, 1}$ durch die beiden Doppelstrahlen von $\left(I_{1}\right)$ harmonisch getrennt ist und also auf dem zu $g_{n, 1}$ in $\left(I_{1}\right)$ zugepaarten $g_{n, 2^{n}}$ liegt; und zwar muß dieser zweite Schnittpunkt von $p^{2}$ mit $l$ der Punkt $G_{2 n, 2^{n+1}}$ (nicht $G_{2 n, 2^{n}}$ ) sein, wie man leicht aus dem $p^{2}$ eingeschriebenen vollständigen Viereck $G_{2 n, 1} G_{2 n, 2^{n}} G_{2 n, 2^{n}+1} G_{2 n, 2^{n+1}}$ mit Hilfe einer bekannten Eigenschaft solcher Vierecke entnehmen kann. Ebenso wie die Gerade $l$ müssen aber alle die Geraden, welche je eins der Punktpaare:

1) $G_{2 n, 1} G_{2 n, 2^{n+1}}, G_{2 n, 2} G_{2 n, 2^{n+1}-1}, G_{2 n, 3} G_{2 n, 2^{n+1}-2}, \ldots$ $G_{2 n, 2^{n}} G_{2 n, 2^{n}+1}$

Berliner, Habilitationgschrift. 
verbinden, durch den Schnittpunkt von $p$ mit dem innerhalb $\triangle G_{2 n, 1} P G_{2 n, 2^{n}}$ (und somit auch innerhalb $\triangle G_{2 n, 2} P G_{2 n, 2^{n}-1}$, $G_{2 n, 3} P G_{2 r, 2^{n}-2}$ usw.) liegenden Doppelstrahle von $\left(I_{1}\right)$ gehen. Es bilden demnach die Punktepaare in 1), deren Zeigersumme $\equiv 1\left(\bmod .2^{n+1}\right)$ ist, eine Involution auf $p^{2}$, deren Zentrum auf $p$ liegt und in welcher Involution folglich die (konjugiert-imaginären) Schnittpunkte von $p$ mit $p^{2}$, also die Doppelpunkte der von $A B C$ auf $p$ erzeugten Involution $(p)^{2}$ (Nr. 2) ein Paar bilden.

Weil aber auch die Strahlenpaare

$$
g_{n, 1} g_{n, 1}, g_{n, 2} g_{n, 2^{n}}, g_{n, 3} g_{n, 2^{n}-1}, \ldots,
$$

deren Zeigersumme $\equiv 2\left(\bmod .2^{n}\right)$ ist, eine zweite auf $(P)^{2}$ sich stützende Involution $\left(I_{2}\right)$ bilden, so kann man in analoger Weise zeigen, daß auch die Punktepaare

2) $G_{2 n, 1} G_{2 n, 1}, G_{2 n, 2} G_{2 n, 2^{n+1}}, G_{2 n, 3} G_{2 n, 2 n+1}, \ldots$

$$
G_{2 n, 2^{n}+1} G_{2 n, 2^{n}+1}
$$

deren Zeigersumme $\equiv 2\left(\bmod .2^{n+1}\right)$ ist, eine Involution auf $p^{2}$ bilden, von welcher die Doppelpunkte von $(p)^{2}$ ebenfalls ein Paar sind.

Mithin müssen die $2^{n+1}$ Schnittpunkte von $g_{n, 1}, g_{n, 2}, g_{n, 3}, \ldots g_{n, 2^{n}}$ mit $p^{2}$, von welchen Schnittpunkten die Paare, deren Zeigersumme $\equiv 1\left(\bmod .2^{n+1}\right)$ ist, in der Punktinvolution 1) und die Paare, deren Zeigersumme $\equiv 2\left(\bmod .2^{n+1}\right)$ ist, in der Punktinvolution 2) sind, eine zyklisch-projektive Gruppe auf $p^{2}$ bilden:

$$
\begin{aligned}
& \left.\pi^{\prime}\right) G_{2 n, 1} G_{2 n, 2} G_{2 n, 3} \ldots G_{2 n, 2^{n}} G_{2 n, 2^{n}+1} \ldots G_{2 n, 2^{n+1}} \\
& \bar{\wedge} G_{2 n, 2} G_{2 n, 3} G_{2 n, 4} \ldots G_{2 n, 2^{n}+1} G_{2 n, 2^{n}+2} \ldots G_{2 n, 1}
\end{aligned}
$$

und zwar erster Art, da diese Schnittpunkte in der natürlichen Anordnung in $p^{2}$ aufeinander folgen; und die Koinzidenzpunkte dieser Projektivität $\pi^{\prime}$ bestehen aus dem gemeinsamen Paare jener beiden Punktinvolutionen 1) und 2), also aus den Doppelpunkten von $(p)^{2}$.

Weil nun der Büschel der Polaren um $P$ zu der Punktreihe der Pole auf $p^{2}$ projektiv ist und die Polaren der auf $p^{2}$ liegenden (konjugiert-imaginären) Doppelpunkte von $(p)^{2}$ die (konjugiertimaginären) Doppelstrahlen von $(P)^{2}$ sind (nach Nr. 3, 7 u. 2), so müssen auch die $2^{n+1}$ durch $P$ gehenden Strahlen, deren Pole die auf $p^{2}$ liegenden Punkte $G_{2 n, 1}, G_{2 n, 2}, \ldots G_{2 n, 2^{n+1}}$ sind, deren Pole also von $P$ aus durch die Gruppe $g_{n, 1} g_{n, 2} \ldots g_{n, 2^{n}}$ von $(P)^{2^{n}}$ 
projiziert werden, und welche $2^{n+1}$ Strahlen mithin eine Gruppe in $(P)^{2^{n+1}}$ bilden, eine zyklisch-projektive Gruppe $\left(2^{n+1}\right)$-ten Grades erster Art bilden:

$\left.\Pi^{\prime}\right) \quad g_{2 n, 1} g_{2 n, 2} g_{2 n, 3} \ldots g_{2 n, 2^{n+1}} \pi g_{2 n, 2} g_{2 n, 3} g_{2 n, 4} \ldots g_{2 n, 1}$, und die Koinzidenzstrahlen dieser Projektivität $\Pi^{\prime}$ müssen, weil sie die Polaren der Koinzidenzpunkte von $\pi^{\prime}$, also die Polaren der Doppelpunkte von $(p)^{2}$ sind, die Doppelstrahlen von $(P)^{2}$ sein.

Es gilt somit unsere Behauptung auch für $(P)^{2^{n+1}}$, wenn sie für $(P)^{2^{n}}$ gilt. In derselben Weise kann man zeigen, daß die Behauptung auch für $(P)^{2^{n+1} \cdot 3}$ richtig bleibt, wenn sie für $(P)^{2^{n} \cdot 3}$ richtig ist; sie muß also allgemein gelten, weil sie für $(P)^{2}$ und $(P)^{3}$ gilt, wo $n=1$ bzw. $n=0$ ist und die Systeme $(P)^{2}$ und $(P)^{3}$ mit den von $A B C$ um $P$ erzeugten Involutionen zweiten und dritten Grades identisch sind (nach Nr.19 und 20).

22. Weil die Zyklen einer zyklischen Projektivität $k$-ten Grades eine Involution desselben Grades bilden, wobei die Koinzidenzelemente der Projektivität die beiden $k$-fachen Elemente der Involution sind, so bilden die Gruppen von $(P)^{2^{n}}$ und $(P)^{2^{n} \cdot 3}$ Involutionen $\left(2^{n}\right)$ - bzw. $\left(2^{n} \cdot 3\right)$-ten Grades, welche wir ebenso wie $(P)^{2}$ und $(P)^{3}$ die vom Dreieck $A B C$ um $P$ erzeugten Involutionen $(P)^{2^{n}}$ und $(P)^{2^{n} \cdot 3}$ nennen wollen und deren $\left(2^{n}\right)$ - bzw. $\left(2^{n} .3\right)$-fache Strahlen die Doppelstrahlen von $(P)^{2}$ sind.

Fassen wir nun die Resultate der letzten drei Nummern zusammen, so haben wir:

Satz 12. Es wird in der oben angegebenen Weise vom Dreieck $A B C$

um jeden a uf keiner Seite dieses Dreiecks liegenden Punkt $P$ eine Strahleninvolution

$\left(2^{n}\right)$-ten und eine $\left(2^{n} \cdot 3\right)$-ten Grades (für jeden positiven ganzzahligen Wert von $n$ )

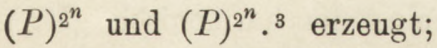
in diesen Involutionen ist jeder der beiden Doppelstrablen von $(P)^{2}$ ein $\left(2^{n}\right)$ bzw. ( $\left.2^{n} \cdot 3\right)$-facher Strahl. auf jeder durch keine Ecke dieses Dreiecks gehenden Geraden $p$ eine Punktinvolution

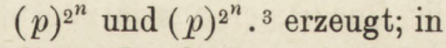
diesen Involutionen ist jeder der beiden Doppelpunkte von $(p)^{2}$ ein $\left(2^{n}\right)$ - bzw. $\left(2^{n} \cdot 3\right)$ facher Punkt. 
Jede dieser Involutionen wird von den Zyklen einer zyklischen Projektivität $\left(2^{n}\right)$ - bzw. $\left(2^{n} \cdot 3\right)$-ten Grades erster Art gebildet. Jede Gruppe in der Involution

$$
(P)^{2^{n}} \text { bzw. }(P)^{2^{n} \cdot 3} \quad \mid \quad(p)^{2^{n}} \text { bzw. }(p)^{2^{n} \cdot 3}
$$

enthält $2^{k}$ bzw. $2^{k}$ und $2^{k} .3$ Gruppen $(0 \leqq k \leqq n)$ der Involution

$$
\begin{array}{c|c}
(P)^{2^{n-k}} \text { bzw. }(P)^{2^{n-k} \cdot 3} & (p)^{2^{n-k}} \text { bzw. }(p)^{2^{n-k} \cdot 3} \\
\text { und }(P)^{2^{n-k}} ; & \text { und }(p)^{2^{n-k}} ;
\end{array}
$$

und zwar bilden je $2^{n-k}$ bzw. $2^{n-k} \cdot 3$ und $2^{n-k}$ Elemente der erstern Gruppe, deren Zeiger zu einer und derselben Zahl kongruent sind (mod. $2^{k}$ bzw. mod. $2^{k}$ und mod. $2^{k} .3$ ), wenn die Ordnung der Elementenzeiger mit der natürlichen Anordnung in einem der beiden Sinne im Gebilde übereinstimmt, eine der letztern Gruppen.

Charakteristisch für die Involutionen $(P)^{2^{n}}$ und $(P)^{2^{n} \cdot 3}$ ist, daß die Pole einer jeden ihrer Strahlengruppen von $P$ aus durch eine Strahlengruppe von $(P)^{2^{n-1}}$ bzw. $(P)^{2^{n-1} \cdot 3}$ projiziert werden, $(p)^{2^{n}}$ und $(p)^{2^{n} .3}$ ist, daß die Polaren einer jeden ihrer Punktgruppen von $p$ in einer Punktgruppe von $(p)^{2^{n-1}}$ bzw. $(p)^{2^{n-1} \cdot 3}$ geschnitten werden,

welche letztere Gruppe die Repräsentationsgruppe in $(P)^{2^{n-1}}$ bzw. $(P)^{2^{n-1} \cdot 3} \mid$ in $(p)^{2^{n-1}}$ bzw. $(p)^{2^{n-1} \cdot 3}$ der erstern ist.

Daß diejenigen $2^{n-k}$ Elemente, deren Zeiger zu einer und derselben Zahl kongruent sind $\left(\bmod .2^{k}\right)$, eine Gruppe bilden, ergibt sich aus der Bemerkung, daß zwischen zwei aufeinanderfolgenden Elementen einer zyklisch-projektiven Gruppe erster Art, also zwischen zwei in der natürlichen Anordnung im Gebilde aufeinanderfolgenden Elementen der Gruppe, nur ein Element aus jeder andern Gruppe derselben zyklischen Projektivität liegt.

Anmerkung. In den Involutionen $(P)^{4}$ und $(p)^{4}$ bestehen die Quadrupel aus je zwei durcheinander harmonisch getrennten Paaren von $(P)^{2}$ bzw. $(p)^{2}$; dies folgt auch daraus, daß die Pole eines Quadrupels von $(P)^{4}$, welche Pole auf $p^{2}$ liegen und von $P$ aus durch ein Strahlenpaar in $(P)^{2}$, also (Nr.2) durch zwei in bezug auf $p^{2}$ konjugierte Gerade projiziert werden, vier harmo- 
nische Punkte auf $p^{2}$ sein müssen. In den Involutionen $(P)^{6}$ und $(p)^{6}$ bestehen die Gruppen aus je drei, ebenso wie $p_{A} p_{A}^{\prime}, p_{B} p_{B}^{\prime}$, $p_{C} p_{C}^{\prime}$, harmonisch beschaffenen Paaren von $(P)^{2}$ bzw. $(p)^{2}$ und zugleich aus zwei zueinander harmonischen Tripeln von $(P)^{3}$ bzw. $(p)^{3}$

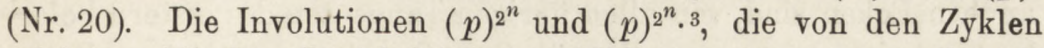
solcher zyklischer Projektivitäten gebildet werden, deren Koinzidenzpunkte die Doppelpunkte von $(p)^{2}$ sind, werden aus denjenigen Punkten, aus denen $(p)^{2}$ durch je eine rechtwinklige Strahleninvolution projiziert wird, durch je eine Involution der regelmäßigen $\left(2^{n}\right)$ - bzw. $\left(2^{n} \cdot 3\right)$-Strahlen projiziert. Dasselbe gilt auch dann, wenn an Stelle von $(p)^{2^{n}},(p)^{2^{n}} \cdot 3$ und $(p)^{2}$ die Projektionen von $(P)^{2^{n}},(P)^{2^{n} \cdot{ }^{3}}$ und $(P)^{2}$ auf irgendeine Gerade treten. Dies bietet ein zweites (s. o. Nr.19) Mittel dar, um die sämtlichen Elemente einer Gruppe von $(P)^{2^{n}},(P)^{2^{n} \cdot 3},(p)^{2^{n}},(p)^{2^{n} \cdot 3}$ aufzufinden, wenn auch nur ein Element derselben Gruppe gegeben ist. $\mathrm{Zu}$ gleich erkennt man hierdurch, daß, wenn die beiden von $A B C$ um einen Punkt und auf einer Geraden erzeugten Involutionen zweiten Grades perspektiv sind, dann auch die beiden von $A B C$ um denselben Punkt und auf derselben Geraden erzeugten Involutionen $2^{n}$ )-ten bzw. (2n. 3)-ten Grades perspektiv sind. Der Satz 3 (Nr. 10) (geht somit über in

Satz 13. Die vom Dreieck $A B C$ um einen Punkt $P$ erzeugten Strahleninvolutionen $(P)^{2^{n}}$ und $(P)^{2^{n} \cdot 3}$ sind außer zu den von $A B C$ auf der Polare $p$ von $P$ erzeugten Punktinvolutionen $(p)^{2^{n}}$ bzw. $(p)^{2^{n} \cdot 3}$ nur noch zu den von $A B C$ auf den sämtlichen Tangenten $q$ des Polarkegelschnitts $P^{2}$ von $p$ erzeugten Punktinvolutionen $(q)^{2^{n}}$ bzw. $(q)^{2^{n} \cdot 3}$ perspektiv.

auf einer Geraden $p$ erzeugten Punktinvolutionen $(p)^{2^{n}}$ und $(p)^{2^{n} \cdot 3}$ sind außer zu den von $A B C$ um den Pol $P$ von $p$ erzeugten Strahleninvolutionen $(P)^{2^{n}}$ bzw. $(P)^{2^{n} \cdot{ }^{3} \text { nur }}$ noch zu den von $A B C$ um die sämtlichen Punkte $G$ des Polarkegelschnitts $p^{2}$ von $P$ erzeugten Strahleninvolutionen $(G)^{2^{n}}$ bzw. $(G)^{2^{n} \cdot 3}$ perspektiv.

23. Doch zeichnen sich Pol und Polare und diejenigen drei Tangenten des Polarkegelschnitts der Polare, welche die vierten harmonischen zu je einer der Seiten $a, b, c$ von $A B C$ (welche Seiten zugleich Tangenten desselben Polarkegelschnitts sind) in 
bezug auf die beiden andern Seiten sind, und diejenigen drei Punkte des Polarkegelschnitts des Pols, welche die vierten harmonischen zu je einer der Ecken von $A B C$ (welche Ecken zugleich auf diesem Polarkegelschnitt liegen) in bezug auf die beiden andern Ecken sind, von allen übrigen Tangenten des erstern Polarkegelschnitts bzw. von allen übrigen Punkten des letztern Polarkegelschnitts im Folgenden aus (siehe weiter unten Nr. 27); es gilt nämlich der

Satz 14. Der Strahlenbüschel um $P$, welcher von den Repräsentanten der Strahlengruppen in $(P)^{2^{n}}$ (wo $n$ jeden positiven ganzen Wert annehmen darf) gebildet wird, und diejenige Punktreihe auf der Polare $p$ von $P$, welche von den Repräsentanten der Punktgruppen in $(p)^{2^{n}}$ gebildet wird, liegen zueinander involutorisch, wenn in diesen beiden Gebilden solche Elemente einander zugewiesen werden, die Repräsentanten inzidenter Gruppen in $(P)^{2^{n}}$ und $(p)^{2^{n}}$ sind; und zwar werden die hierdurch um $P$ und auf $p$ entstehenden Involutionen mit $(P)^{2}$ und $(p)^{2}$ identisch sein. Das Nämliche gilt von

demselben Strahlenbüschel um $P$ und einer derjenigen Punktreihen auf den drei Tangenten $p_{a}^{\prime}, p_{b}^{\prime}, p_{c}^{\prime}$ des $\mathrm{Po}_{0}$ larkegelschnitts $P^{2}$ von $p$, welche von den Repräsentanten der Punktgruppen in den von $A B C$ erzeugten,

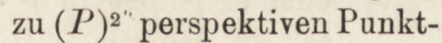
involutionen $\left(p_{a}^{\prime}\right)^{2^{n}}, \quad\left(p_{b}^{\prime}\right)^{2^{n}}$, $\left(p_{c}^{\prime}\right)^{n}$ gebildet werden, wo $p_{a}^{\prime}, p_{b}^{\prime}, p_{c}^{\prime}$ an $P^{2}$ die vierten harmonischen Tangenten zu je einer der Dreieckseiten $a, b, c$ in bezug auf die beiden andern Seiten sind.

Es ist also der $\mathrm{Re}$ präsentant einer Strahlengruppe in $(P)^{2^{n}}$ von dem derselben Punktreihe auf $p$ und einem derjenigen Strahlenbüschel um die dreiPunkte $P_{A}^{\prime}, P_{B}^{\prime}, P_{C}^{\prime}$ des Polarkegelschnitts $p^{2}$ von $P$, welche von den Repräsentanten der Strahlengruppen in den von $A B C$ erzeugten, $\mathrm{zu}(p)^{2^{n}}$ perspektiven Strahleninvolutionen $\left(P_{A}^{\prime}\right)^{2^{n}},\left(P_{B}^{\prime}\right)^{2^{n}},\left(P_{C}^{\prime}\right)^{2^{n}}$ gebildet werden, wo $P_{A}^{\prime}, \mathrm{P}_{B}^{\prime}$, $P_{C}^{\prime}$ in $p^{2}$ die vierten harmonischen Punkte zu je einer der Ecken $A, B, C$ in bezug auf die beiden andern Ecken sind.

Es ist also der Repräsentant einer Punktgruppe in $(p)^{2^{n}}$ von dem 
Repräsentanten der mitjener Strahlengruppe inzidenten Punktgruppe in bzw. $(p)^{2^{n} \text {, }}$ $\left(p_{a}^{\prime}\right)^{2^{n}},\left(p_{b}^{\prime}\right)^{2^{n}},\left(p_{c}^{\prime}\right)^{2^{n}}$ durch die Doppelelemente von $(P)^{2}$ und ebenso durch die von bzw. $(p)^{2},\left(p_{a}^{\prime}\right)^{2},\left(p_{b}^{\prime}\right)^{2},\left(p_{c}^{\prime}\right)^{2}$ harmonisch getrennt.

Ferner ist das Repräsentationstripel einer Strahlengruppe in $(P)^{2^{n} \cdot 3} \mathrm{zu}$ dem Repräsentationstripel der mit jener Strahlengruppe inzidenten Punktgruppe in bzw. $(p)^{2^{n} \cdot 3}, \quad\left(p_{a}^{\prime}\right)^{2^{n} \cdot 3}, \quad\left(p_{b}^{\prime}\right)^{2^{n} \cdot 3}$, $\left(p_{c}^{\prime}\right)^{2^{n} \cdot 3}$ harmonisch.
Repräsentanten der mit jener Punktgruppe inzidenten Strahlengruppe in bzw. $(P)^{2^{n}},\left(P_{A}^{\prime}\right)^{2^{n}},\left(P_{B}^{\prime}\right)^{2^{n}},\left(P_{\mathrm{C}}^{\prime}\right)^{2^{n}}$ durch die Doppelelemente von $(p)^{2}$ und ebenso durch die von bzw. $(P)^{2},\left(P_{A}^{\prime}\right)^{2}$, $\left(P_{B}^{\prime}\right)^{2},\left(P_{C}^{\prime}\right)^{2}$ harmonisch getrennt.

Ferner ist das Repräsentationstripel einer Punktgruppe in $(p)^{2^{n} \cdot 3} \mathrm{zu}$ dem Repräsentationstripeldermit jener Punktgruppe inzidenten Strahlengruppe in bzw. $(P)^{2^{n} \cdot 3},\left(P_{A}^{\prime}\right)^{2^{n} \cdot 3},\left(P_{B}^{\prime}\right)^{2^{n} \cdot 2}$ $\left(P_{C}^{\prime}\right)^{2^{n} \cdot 3}$ harmonisch.

Beweis. Wie mit Hilfe des Satzes 2 (Nr. 8) leicht einzusehen ist, muß der Strahlenbüschel der Repräsentanten der Paare in $(P)^{2}$ zu der Punktreihe der Repräsentanten der Paare in $(p)^{2}$ projektiv sein mit solchen Elementen als entsprechenden, welche Repräsentanten inzidenter Paare in $(P)^{2}$ und $(p)^{2}$ sind. In dieser Projektivität entsprechen nun den drei durch $P$ gehenden Ecktransversalen von $A B C$, nämlich: $p_{A}, p_{B}$ und $p_{C}$, welche die Repräsentanten der Strahlenpaare $p_{A} p_{A}^{\prime}, p_{B} p_{B}^{\prime}, p_{C} p_{C}^{\prime}$ von $(P)^{2}$ sind (nach Nr.7), die drei Schnittpunkte der Seiten von $A B C$ mit $p$, nämlich: $P_{a}, P_{b}$ und $P_{c}$, welche die Repräsentanten der Punktepaare $P_{a}^{\prime} P_{a}, P_{b}^{\prime} P_{b}$ und $P_{c}^{\prime} P_{c}$ von $(p)^{2}$ sind; da diese drei Punktepaare mit jenen drei Strahlenpaaren inzident sind (Nr. 5). Die drei Ecktransversalen $p_{A}, p_{B}, p_{C}$ gehen aber durch die drei zu $P_{a}, P_{b}, P_{c}$ in der Involution $(p)^{2}$ zugepaarten Punkte $P_{a}^{\prime}, P_{b}^{\prime}$, $P_{c}^{\prime}$; mithin muß dies durchweg geschehen und jeder Strahl von $P$ geht durch denjenigen Punkt von $p$, welcher zu dem demselben Strahle von $P$ in der Projektivität entsprechenden Punkte zugepaart ist in $(p)^{2}$. Damit ist der vorstehende Satz für $(P)^{2}$ und $(p)^{2}(n=1)$ bewiesen.

Nach dem soeben Bewiesenen muß nun der die Pole eines Strahlenpaares in $(P)^{2}$ von $P$ aus projizierende Strahl (der der 
Repräsentant jenes Strahlenpaares ist) von demjenigen Punkte, in dem die Polaren des mit jenem Strahlenpaare inzidenten Punktepaares in $(p)^{2}$ sich schneiden (welcher Punkt also der Repräsentant dieses Punktepaares ist), durch die Doppelelemente von $(P)^{2}$ und $(p)^{2}$ harmonisch getrennt sein. Mithin inzidiert das die Pole eines Strahlenquadrupels in $(P)^{4}$ von $\boldsymbol{P}$ aus projizierende Strahlenpaar in $(P)^{2}$ mit demjenigen Punktepaar in $(p)^{2}$, in welchem die Polaren des mit jenem Strahlenquadrupel inzidenten Punktquadrupels der zu $(P)^{4}$ perspektiven $(p)^{4}$ sich schneiden. Nun enthält aber jede Strahlengruppe in $(P)^{2^{n}}(n \geqq 2) 2^{n-2}$ Strahlenquadrupel von $(P)^{4}$ und die mit ihr inzidente Punktgruppe in der zu $(P)^{2^{n}}$ perspektiven $(p)^{2^{n}} 2^{n-2}$ Punktquadrupel von $(p)^{4}$, von welchen Punktquadrupeln jedes mit einem der $2^{n-2}$ Strahlenquadrupel von $(P)^{4}$ inzident ist. Also muß die die Pole einer Strahlengruppe in $(P)^{2^{n}}$ von $P$ aus projizierende Strahlengruppe in $(P)^{2^{n-1}}$ mit derjenigen Punktpruppe in $(p)^{2^{n-1}}$ inzidieren, in welcher die Polaren der mit der ersten Strahlengruppe inzidenten Punktgruppe von $(p)^{2^{n}}$ sich schneiden; oder mit andern Worten (nach Nr. 19): die Repräsentationsgruppen in $(P)^{2^{n-1}}$ und $(p)^{2^{n-1}}$ inzidenter Gruppen in $(P)^{2^{n}}$ und $(p)^{2^{n}}$ sind gleichfalls inzident. Mithin inzidieren auch die Repräsentationsgruppen in $(P)^{2^{n-2}}$ und $(p)^{2^{n-2}}$, in $(P)^{2^{n-3}}$ und $(p)^{2^{n-3}}$ usw. bis in $(P)^{2}$ und $(p)^{2}$, wenn die repräsentierten Gruppen in $(P)^{2^{n}}$ und $(p)^{2^{n}}$ inzidieren. Folglich muß der Repräsentant einer Strahlengruppe in $(P)^{2^{n}}$ von dem Repräsentanten der mit jener Strahlengruppe inzidenten Punktgruppe in $(p)^{2^{n}}$ durch die Doppelelemente von $(P)^{2}$ und $(p)^{2}$ harmonisch getrennt sein, da diese beiden Repräsentanten zugleich die Repräsentanten derjenigen inzidenten Paare in $(P)^{2}$ und $(p)^{2}$ sind, welche die Repräsentationspaare in $(P)^{2}$ und $(p)^{2}$ jener inzidenten Gruppen in $(P)^{2^{n}}$ und $(p)^{2^{n}}$ sind; damit ist der vorstehende Satz für $(P)^{2^{n}}$ und $(p)^{2^{n}}$, auch wenn $n>1$ ist, bewiesen.

Weil nun eine Gruppe in $(P)^{2^{n} \cdot 3}$ aus drei Gruppen von $(P)^{2^{n}}$ besteht, und das Repräsentationstripel der erstern Gruppe aus den drei Repräsentanten der drei letztern besteht (Nr.20), und dasselbe von einer Gruppe in der zu $(P)^{2^{n} \cdot 3}$ perspektiven $(p)^{2^{n} \cdot 3}$ gilt, so muß, nach dem eben Bewiesenen, das Repräsentationstripel einer Strahlengruppe in $(P)^{2^{n} \cdot 3}$ durch dasjenige Punkttripel in $(p)^{3}$ gehen, dessen Punkte den Punkten des Repräsentationstripels der mit jener Strahlengruppe inzidenten Punkt- 
gruppe von $(p)^{2^{n} \cdot 3}$ zugepaart sind in $(p)^{2}$ und welches Punkttripel also zu dem letztern Repräsentationstripel harmonisch ist (Nr. 4). Der vorstehende Satz ist also für $P$ und $p$ bewiesen.

In ganz analoger Weise kann man zeigen, daß dieser Satz auch für $P$ und eine der drei Tangenten $p_{a}^{\prime}, p_{b}^{\prime}, p_{c}^{\prime}$ von $P^{2}$ und für $p$ und einen der drei Punkte $P_{A}^{\prime}, P_{B}^{\prime}, P_{C}^{\prime}$ von $p^{2}$ gilt. Denn $(P)^{2^{n}}$ und $(P)^{2^{n} \cdot 3}$ sind etwa zu $\left(p_{a}^{\prime}\right)^{2^{n}}$ und $\left(p_{a}^{\prime}\right)^{2^{n} \cdot 3}$ perspektiv (Satz 13), und die Tangente $p_{a}^{\prime}$, die am Polarkegelschnitt $P^{2}$ von $p$ die vierte harmonische zu der Tangente $a$, der Polare von $P_{a} \equiv p a$, in bezug auf die Tangenten $b$ und $c$, die Polaren von $P_{b} \equiv p b$ und $P_{c} \equiv p c$, ist, muß die Polare des auf der Ecktransversale $p_{A} \equiv P A$ liegenden Punktes $P_{a}^{\prime}$ von $p$ (Nr.5) sein, welcher Punkt $P_{a}^{\prime}$ der vierte harmonische zu $P_{a}$ in bezug auf $P_{b}$ und $P_{c}$ ist. Es geht also die Ecktransversale $p_{A} \equiv P_{a}^{\prime} A$ durch den in $\left(p_{a}^{\prime}\right)^{2}$ zum Schnittpunkte $p_{a}^{\prime} a$ zugepaarten Punkt (nach Nr.5) und mithin gehen $p_{B}$ und $p_{C}$ durch die in $\left(p_{a}^{\prime}\right)^{2}$ zu den Schnittpunkten $p_{a}^{\prime} c$ und $p_{a}^{\prime} b$ zugepaarten Punkte; da $p_{A} p_{B} p_{C}$ ein Tripel in $(P)^{3}$ und die in $\left(p_{a}^{\prime}\right)^{2}$ zu $p_{a}^{\prime} a, p_{a}^{\prime} b, p_{a}^{\prime} c$ zugepaarten Punkte ein mit $\left(p_{a}^{\prime} a\right)\left(p_{a}^{\prime} b\right)\left(p_{a}^{\prime} c\right)$ gleichen Sinn habendes Tripel in der zu $(P)^{3}$ perspektiven $\left(p_{a}^{\prime}\right)^{3}$ bilden (Nr. 4) und der durch $A B C$ um $P$ festgelegte Sinn zu dem auf $p_{a}^{\prime}$ festgelegten entgegengesetzt ist (Nr.11). 
Zweiter Abschnitt ${ }^{1}$ ).

\section{Prozels der Repräsentantenbildung.}

\section{$\S 5$.}

24. Wie wir oben (Nr.19) sahen, werden in der von $A B C$ in einem Gebilde erster Stufe, welches keine Ecke bzw. Seite von $A B C$ enthält, erzeugten Involution $\left(2^{n}\right)$-ten Grades durch irgendein Element desselben Gebildes zwei Gruppen bestimmt, nämlich: eine, deren Repräsentant jenes Element ist, und eine zweite, der jenes Element angehört.

Nun wollen wir die Frage aufwerfen:

Gibt es im Gebilde erster Stufe Elemente, bei denen die je zwei durch sie in der Involution $\left(2^{n}\right)$-ten Grades bestimmten Gruppen zusammenfallen; oder mit anderen Worten: gibt es Elemente, welche den durch sie repräsentierten Gruppen der Involution $\left(2^{n}\right)$-ten Grades angehören?

Dieser Frage können wir noch eine andere Form geben. $\mathrm{Zu}$ diesem Zweck bemerken wir folgendes:

Indem wir den Repräsentanten einer Gruppe in einer Involution $\left(2^{n}\right)$-ten Grades als den Repräsentanten eines jeden der $2^{n}$ dieser Gruppe angehörenden Elemente ansehen, können wir von einem ersten, zweiten, dritten und überhaupt von einem $n$-ten Repräsentanten eines Elementes sprechen. Es hat nämlich in einem Gebilde erster Stufe, welches keine Ecke bzw. Seite von $A B C$ enthält, jedes Element $Q$, als einem Paare in der von $A B C$ im Gebilde erzeugten Involution zweiten Grades angehörig, einen ersten Repräsentanten $Q^{(1)}$, nämlich den Repräsentanten dieses Paares; dasselbe Element $Q$, als einem Quadrupel in der Involution vierten Grades angehörig, hat einen zweiten

1) In diesem Abschnitt kommen nur reelle Gebilde und Elemente in Betracht. 
Repräsentanten $Q^{(2)}$, nämlich den Repräsentanten dieses Quadrupels, und ebenso, als einer Gruppe in der Involution achten Grades angehörig, einen dritten $Q^{(3)}$ und überhaupt, als einer Gruppe in der Involution $\left(2^{n}\right)$-ten Grades, einen $n$-ten Repräsentanten $Q^{(n)}$. Jedes Element hat also nur einen $n$-ten Repräsentanten (für jeden positiven ganzen Wert von $n$ ), dagegen werden von jedem Elemente, als $n$-ten Repräsentanten, $2^{n}$ Elemente, nämlich die $2^{n}$ Elemente einer Gruppe in der Involution $\left(2^{n}\right)$-ten Grades repräsentiert.

Um den ersten, zweiten usw. Repräsentanten eines Elementes zu ermitteln, haben wir, wenn das Element etwa ein Strahl von $P$ ist, $P$ mit dem Pole dieses Strahles durch eine Gerade zu verbinden, darauf mit dem Pole dieser Verbindungsgeraden durch eine zweite Gerade, sodann mit dem Pole der zweiten Verbindungsgeraden durch eine dritte usf.; die erste, zweite usw. Verbindungsgerade werden dann der erste, zweite usw. Repräsentant jenes Strahles von $P$ sein (nach Nr.19).

Der $n$-te Repräsentant eines Elementes ist also zugleich der erste, der zweite und überhaupt der $k$-te Repräsentant $(k<n)$ des $(n-1)$-ten bzw. des $(n-2)$-ten bzw. des $(n-k)$-ten Repräsentanten desselben Elementes.

Die aufgeworfene Frage ist nun mit folgender gleichbedeutend:

Gibt es in einem Gebilde erster Stufe, welches keine Ecke bzw. Seite von $A B C$ enthält, Elemente, die mit ihren $n$-ten Repräsentanten zusammenfallen?

25. Ohne die Allgemeinheit zu beeinträchtigen, können wir uns auf den Fall beschränken, wo das Gebilde ein Strahlenbüschel um den auf keiner Seite von $A B C$ liegenden Punkt $P$ ist. Allererst erkennt man, daß jeder der drei durch $P$ gehenden Ecktransversalen von $A B C$ mit seinem $n$-ten Repräsentanten (für jeden Wert von $n$ ) zusammenfällt. Denn der Pol einer durch $P$ gehenden Ecktransversalen ist die auf derselben liegende Ecke; es fällt also die $P$ mit diesem Pole verbindende Gerade, der erste Repräsentant jener Ecktransversalen, und mithin auch der zweite, dritte usw. Repräsentant mit jener Ecktransversalen zusammen.

Ferner sieht man auch ein, daß außer den Ecktransversalen kein Strahl von $P$ mit seinem ersten oder zweiten Repräsentanten 
zusammenfallen $\operatorname{kann}^{1}$ ). Denn kein durch keine Ecke von $A B C$ gehender Strahl von $P$ kann seinen Pol enthalten (Nr.1) und somit mit seinem ersten Repräsentanten zusammenfallen. Sollte aber ein solcher Strahl mit seinem zweiten Repräsentanten zusammenfallen, so würde dann jeder der beiden Strahlen, nämlich der mit seinem zweiten Repräsentanten zusammenfallende Strahl und sein erster Repräsentant, den Pol des andern enthalten, was unmöglich ist; da die Polare eines Punktes ganz außerhalb des von den Polen der durch diesen Punkt gehenden Strahlen gebildeten Kegelschnitts, des Polarkegelschnitts dieses Punktes, liegt (Nr. 2).

Dual fällt jeder der drei Schnittpunkte einer Geraden mit den Seiten von $A B C$ mit seinem $n$-ten Repräsentanten zusammen (für jeden Wert von $n$ ) und kein von diesen Schnittpunkten verschiedener Punkt der Geraden fällt mit seinem ersten oder zweiten Repräsentanten zusammen.

Es fragt sich also nur noch: ob es außer den Ecktransversalen noch Strahlen von $P$ gibt, die mit ihren $n$-ten Repräsentanten zusammenfallen, wo $n \geqq 3$ ist.

26. Um nun die aufgeworfene Frage vollständig beantworten zu können, bedürfen wir des nächsten Satzes, welcher uns erlauben wird, den $n$-ten Repräsentanten eines Elementes direkt, ohne Vermittelung der früheren Repräsentanten anzugeben und welchem Satze wir folgendes voranschicken.

Es sei $p$ irgendeine durch keine Ecke von $A B C$ gehende Gerade, $Q$ irgendein Punkt auf ihr, $P_{a}$ ihr Schnittpunkt mit der Seite $a$ von $A B C$ und $R$ einer der beiden Punkte, aus denen die von $A B C$ auf $p$ erzeugte Involution $(p)^{2}$ durch je eine rechtwinklige Strahleninvolution projiziert wird. Ferner sei $P$ der Pol von $p$, $g_{i}$ irgendein durch $P$ gehender Strahl, $p_{A}$ die $P$ mit der Ecke $A$ von $A B C$ verbindende Ecktransversale und die Punkte $Q_{i}$ und $P_{a}^{\prime}$ seien die Spuren von $g_{i}$ und $p_{A}$ auf $p$. Wir wollen dann den Winkel, durch welchen diejenige Strecke, deren Anfangs- und Endpunkt

1) Dies gilt aber nicht mehr von imaginären Strahlen von $P$; denn, wie nach Nr. 3 (S. 5), 7 (S. 12, 13), 2 (S. 2), 5 und 26 (S. 46) leicht einzusehen, ist jeder der beiden konjugiert-imaginären Doppelstrahlen von $(P)^{2}$ der erste Repräsentant des andern, und mithin fällt jeder dieser beiden Doppelstrahlen mit seinem zweiten Repräsentanten zusammen. 
$P_{a}$ und $Q$ sind, von $R$ aus projiziert wird, den Ordinatenwinkel von $Q$ nennen; und zwar ist dieser Ordinatenwinkel (der immer vom Schenkel $P_{a} R$ aus gemessen werden soll) entweder der Winkel $P_{a} R Q$ selbst, oder sein Nebenwinkel (welcher letztere von $P_{a} R$ und der Verlängerung von $Q R$ gebildet wird und welcher also zu $P_{a} R Q$ entgegengesetzten Sinn hat), je nachdem wir uns denken, daß $Q$ von $P_{a}$ aus nach seiner jetzigen Lage auf der endlichen Strecke zwischen $P_{a}$ und $Q$ sich bewegt habe, oder im entgegengesetzten Sinne auf der unendlichen. Ingleichen wollen wir den Winkel, durch welchen diejenige Strecke, deren Anfangs - und Endpunkt $P_{a}^{\prime}$ und $Q_{i}$ sind, von $R$ aus projiziert wird, den Ordinatenwinkel von $g_{i}$ nennen; und zwar ist dieser Ordinatenwinkel (der immer von $P_{a}^{\prime} R$ aus gemessen werden soll) entweder der Winkel $P_{a}^{\prime} R Q_{i}$ selbst, oder sein Nebenwinkel (welcher letztere von $P_{a}^{\prime} R$ und der Verlängerung von $Q_{i} R$ gebildet wird und welcher also zu $P_{a}^{\prime} R Q_{i}$ entgegengesetzten Sinn hat), je nachdem wir uns denken, daß $g_{i}$ von $p_{A}$ aus (und somit $Q_{i}$ von $P_{a}^{\prime}$ aus) nach seiner jetzigen Lage in dem einen oder im entgegengesetzten Sinne sich bewegt habe. Denken wir aber uns, daß der Punkt $Q$ von $P_{a}$ aus, bzw. der Strahl $g_{i}$ von $p_{A}$ aus nach seiner jetzigen Lage erst dann gekommen sei, nachdem er die ganze Gerade $p$, bzw. den ganzen Büschel um $P k$-mal durchlaufen habe, so wird der Ordinatenwinkel von $Q$ bzw. von $g_{i}$ der Winkel $P_{a} R Q+k \pi$ bzw. $P_{a}^{\prime} R Q_{i}+k \pi$ oder ihre Nebenwinkel $+k \pi$, abhängig vom gedachten Sinne der Bewegung von $Q$ bzw. von $g_{i}$, sein. Dabei soll einer der beiden Sinne auf $p$ (bzw. im Büschel um $P$ ) und also der diesem entsprechende im Büschel um $R$ als der positive und der andere als der negative gelten, und demnach werden wir zwischen positiven und negativen Ordinatenwinkeln unterscheiden.

Der Ordinatenwinkel (mit Vorzeichen versehen) eines Elementes gibt also nicht nur das Element selbst an, sondern auch den Weg, den dasselbe Element bei seiner Bewegung von $P_{a}$ aus bzw. von $p_{A}$ aus nach seiner jetzigen Lage zurückgelegt hat.

Es gilt nun der

Satz 15. Der Ordinatenwinkel des $n$-ten Repräsentanten eines Elementes ist (absolut genommen) $2^{n}$-mal größer als der Ordinatenwinkel des nämlichen Elementes; diese beiden Ordinatenwinkel haben gleichen oder entgegengesetzten Sinn, 
je nachdem $n$ gerade oder ungerade ist. Ist also $\omega$ der Ordinatenwinkel des Elementes und $\omega^{(n)}$ der des $n$-ten Repräsentanten, so ist

$$
\omega^{(n)}=(-2)^{n} \omega .
$$

Beweis. Durchläuft ein Strahl $g$ im Büschel um $P$ ein Intervall, welches von zwei aufeinanderfolgenden Strahlen $g_{k, i}$ und $g_{k, i+1}$ einer Gruppe in $(P)^{2^{k}}$ begrenzt wird und welches Intervall mehr keinen Strahl derselben Gruppe enthält, stetig, so durchläuft der Pol $G$ von $g$ auf dem Polarkegelschnitt $p^{2}$ von $P$ das Intervall, welches von den beiden Polen $G_{k, i}$ und $G_{k, i+1}$ von $g_{k, i}$ und $g_{k, i+1}$ begrenzt wird und welches mehr keinen Pol eines Strahles derselben Gruppe enthält, ebenfalls stetig; da die Punktreihe der Pole zu dem Büschel der Polaren projektiv ist (Nr. 2). Alsdann nuß aber auch der $G$ von dem innerhalb $p^{2}$ liegenden Punkte $P$ aus projizierende Strahl, der erste Repräsentant $g^{(1)}$ von $g$, im Büschel um $P$ dasjenige Intervall einmal stetig durchlaufen, welches von $g_{(k, i)}^{(1)}$ und $g_{(k, i+1)}^{(1)}$, den beiden ersten Repräsentanten der zwei aufeinanderfolgenden Strahlen $g_{k, i}$ und $g_{k, i+1}$ der Gruppe in $(P)^{2^{k}}$, begrenzt wird und mehr keinen ersten Repräsentanten eines Strahles derselben Gruppe von $(P)^{2^{k}}$ enthält, welches Intervall also von zwei Strahlen einer Gruppe in $(P)^{2^{k-1}}$ (nach Satz 12 in Nr. 22) begrenzt wird und mehr keinen Strahl dieser Gruppe in $(P)^{2^{k-1}}$ enthält; und zwar sind die dieses Intervall begrenzenden ersten Repräsentanten $g_{(k, i)}^{(1)}$ und $\left.g_{(k, i}^{(1)}+1\right)$ zwei a ufeinanderfolgende Strahlen der Gruppe in $(P)^{2^{k-1}}$, da sie ein außer ihnen mehr keinen Strahl derselben Gruppe in $(P)^{2^{k-1}}$ enthaltendes Intervall um $P$ abgrenzen. (Ist $k=1$, so reduziert sich eine Gruppe in $(P)^{2^{k-1}}=(P)^{1}$ auf einen einzigen Strahl von $P$, und das Intervall zwischen zwei aufeinanderfolgenden Strahlen einer Gruppe in $(P)^{1}$ macht den ganzen Büschel um $P$ aus.) Nunmehr müssen aber die Bewegungen von $g$ und $g^{(1)}$ einander entgegengesetzt sein. Denn, wenn $g$ in einem und demselben Sinne um $P$ sich dreht, muß $g^{(1)}$ (der den Pol $G$ von $g$ mit dem innerhalb des Polarkegelschnitts $p^{2}$ liegenden Punkte $P$ verbindet) auch in einem und demselben Sinne um $P$ sich drehen; der letztere Sinn muß also zum erstern immer entgegengesetzt sein, wie dies beim Durchgang durch eine Ecke von $A B C$ der Fall ist, wo in diesem Moment $g$ und $g^{(1)}$ zusammenfallen (Nr. 25) und alsbald, da die Polare $g$ nicht in das ihren Pol $G$ enthaltende Dreiecks- 
gebiet eindringen kann (Nr.1), in entgegengesetzten Richtungen auseinander gehen. Wenn also $g i$ aufeinanderfolgende Intervalle, von denen jedes von zwei aufeinanderfolgenden Strahlen einer und derselben Gruppe in $(P)^{2^{k}}$ begrenzt wird und mehr keinen Strahl derselben Gruppe enthält, stetig durchlaufen wird, so wird $g^{(1)} i$ aufeinanderfolgende Intervalle, von denen jedes von zwei aufeinanderfolgenden Strahlen einer und derselben Gruppe in $(P)^{2^{k-1}}$ begrenzt wird und mehr keinen StrahI derselben Gruppe in $(P)^{2^{k-1}}$ enthält, im entgegengesetzten Sinne stetig durchlaufen.

Weil aber die Spuren zweier aufeinanderfolgender Strahlen einer Gruppe in $(P)^{2^{k}}$ bzw. in $(P)^{2^{k-1}}$ auf $p$, der Polare von $P$, aus dem Punkte $R$, aus dem $(p)^{2}$ durch eine rechtwinklige Strahleninvolution projiziert wird, durch zwei miteinander einen Winkel $\frac{\pi}{2^{k}}$ bzw. $\frac{\pi}{2^{k-1}}$ bildende Strahlen projiziert werden (Anmerkung in Nr. 22), so geht das letzte Ergebnis ins folgende über: Durchläuft ein Strahl $g$ von $P$ ein Intervall, dessen Spur auf $p$ von $R$ aus durch einen Winkel $\frac{i \pi}{2^{k}}$ projiziert wird (wo dieser Winkel im Sinne der Bewegung der Spur von $g$ auf $p$ gemessen werden soll), stetig, so durchläuft $g^{(1)}$, der erste Repräsentant von $g$, im entgegengesetzten Sinne ein Intervall um $P$, dessen Spur auf $p$ von $R$ aus durch einen Winkel $-\frac{i \pi}{2^{k-1}}=-2 \frac{i \pi}{2^{k}}$ projiziert wird, stetig; dabei bedeuten $i$ und $k$ beliebige, aber nur ganze Zahlen.

Weil ferner jeder beliebige Winkel $\alpha \pi$, wo $\alpha$ nicht in der Form $\frac{i}{2^{k}}$ darstellbar ist oder sogar irrational ist, zwischen zwei Winkeln $\frac{s \pi}{2^{k}}$ und $\frac{(s+1) \pi}{2^{k}}$ liegt (wo $s$ eine ganze Zahl bedeutet), und weil der Winkel $\frac{\pi}{2^{k}}$ und mithin auch $\frac{\pi}{2^{k-1}}$ dadurch beliebig klein gemacht werden kann, daß $k$ hinreichend groß genommen wird, so zeigt der Übergang zur Grenze, wo $\lim _{k=\infty} \frac{\pi}{2^{k}}=0$, daß, wenn auch $g$ ein Intervall um $P$, dessen Spur auf $p$ von $R$ aus durch einen beliebigen Winkel $\alpha \pi$ projiziert wird, stetig durchläuft, $g^{(1)}$ ein Intervall um $P$, dessen Spur auf $p$ von $R$ aus durch einen Winkel $-2 \alpha \pi$ projiziert wird, im entgegengesetzten Sinne stetig durchläuft. 
Ist nun das von $g$ durchlaufene Intervall von $P$, dessen Spur auf $p$ von $R$ aus durch einen beliebigen Winkel $\alpha \pi$ projiziert wird, von der Ecktransversale $p_{A}$, als Anfangsstrahl, und irgendeinem Strahle $g_{i}$, als Endstrahl, begrenzt, und ist mithin das von $g^{(1)}$ im entgegengesetzten Sinne um $P$ durchlaufene Intervall, dessen Spur auf $p$ von $R$ aus durch den Winkel $-2 \alpha \pi$ projiziert wird, von $p_{A}$ (nach Nr. 25), als Anfangsstrahl, und $g_{i}^{(1)}$ (dem ersten Repräsentanten von $g_{i}$ ), als Endstrahl, begrenzt, so sind $\alpha \pi$ und $-2 \alpha \pi$ die Ordinatenwinkel $\omega_{i}$ bzw. $\omega_{i}^{(1)}$ der Endstrahlen $g_{i}$ und $g_{i}^{(1)}$; mithin muß für jeden beliebigen Strahl $g_{i}$ sein:

$$
\omega_{i}^{(1)}=-2 \omega_{i} .
$$

In ganz analoger Weise kann man zeigen, daß, wenn $\omega$ und $\omega^{(1)}$ die Ordinatenwinkel irgendeines Punktes und dessen ersten Repräsentanten sind,

$$
\omega^{(1)}=-2 \omega .
$$

Hiermit ist der vorstehende Satz für $n=1$ bewiesen; seine allgemeine Gültigkeit kann durch den Schluß von $n$ auf $n+1$ nachgewiesen werden. Gilt nämlich dieser Satz für einen bestimmten Wert von $n$, ist also für diesen Wert von $n$ :

$$
\omega^{(n)}=(-2)^{n} \omega,
$$

so muß, weil der $(n+1)$-te Repräsentant eines Elementes zugleich der erste Repräsentant des $n$-ten Repräsentanten desselben Elementes ist (Nr. 24) und der vorstehende Satz für den ersten Repräsentanten $(n=1)$ schon bewiesen ist, sein:

$$
\omega^{(n+1)}=-2 \omega^{(n)}=-2(-2)^{n} \omega=(-2)^{n+1} \omega,
$$

somit gilt dann dieser Satz auch für $n+1$; nun gilt er für $n=1$, mithin allgemein.

27. Beiläufig bemerken wir, daß der Satz 15 (nach Anmerkung in Nr. 22) auch dann noch richtig bleibt, wenn zur Bestimmung der Ordinatenwinkel der Strahlen von $P$ an Stelle der Spuren dieser Strahlen auf der Polare $p$ von $P$ die Spuren derselben Strahlen auf irgendeiner beliebig zu nehmenden Geraden $l$ benutzt werden und an Stelle des oben (Nr. 26) angegebenen Punktes $R$ einer derjenigen Punkte benutzt wird, aus denen die Projektion von $(P)^{2}$ auf $l$ durch je eine rechtwinklige Strahleninvolution projiziert wird, insbesondere wenn an Stelle der Spuren der Strahlen von $P$ auf $p$ die Spuren derselben auf irgendeiner 
Tangente $q$ des Polarkegelschnitts $P^{2}$ von $p$ benutzt werden und an Stelle von $R$ derjenige Punkt $S$ benutzt wird, aus dem die von $A B C$ auf $q$ erzeugte, zu $(P)^{2}$ perspektive (Satz 3 in Nr. 10) Punktinvolution $(q)^{2}$ durch eine rechtwinklige Strahleninvolution projiziert wird.

Ist nun $g$ irgendein Strahl von $P, K$ seine Spur auf der Tangente $q$ des Polarkegelschnitts $P^{2}$ von $p, Q_{a}$ der Schnittpunkt von $q$ mit der Seite $a$ von $A B C, K_{a}$ die Spur der durch $P$ gehenden Ecktransversale $p_{A}(\equiv P A)$ auf $q$, ferner $g^{(n)}$ der $n$-te Repräsentant von $g$ und $K^{(n)}$ der von $K$, und werden die Tangente $q$ und der obige Punkt $S$ zur Bestimmung der Ordinatenwinkel nicht nur für die Punkte von $q$, sondern auch für die Strahlen von $P$ benutzt, wo jedoch für die Punkte von $q S Q_{a}$ und für die Strahlen von $P S K_{a}$ je als Anfangsstrahl der Messung der Ordinatenwinkel genommen werden muß (Nr. 26), und sind (in diesen Ordinatensystemen) $\boldsymbol{\Omega}, \boldsymbol{\Omega}^{(n)}, \boldsymbol{\Omega}_{a}, \omega$ und $\omega^{(n)}$ der Reihe nach die Ordinatenwinkel von $K, K^{(n)}, K_{a}, g$ und $g^{(n)}$, wo bei der Bestimmung von $\boldsymbol{\Omega}_{a}, \boldsymbol{\Omega}$ und $\omega$ gedacht wird (Nr. 26), daß $K_{a}$ und $K$ von $Q_{a}$ aus und $g$ von $p_{A}$ aus nach ihren jetzigen Lagen sämtlich in einem und demselben Sinne, nämlich im Sinne der endlichen Strecke $Q_{a} K_{a}$ sich bewegt haben, so sind entweder

oder

$$
\omega=\Omega-\Omega_{a} \quad \text { und } \quad \omega^{(n)}=(-2)^{n}\left(\Omega-\Omega_{a}\right) \text {, }
$$

$$
\omega=\Omega-\Omega_{a}+\pi \text { und } \omega^{(n)}=(-2)^{n}\left(\Omega-\Omega_{a}+\pi\right),
$$

je nachdem $K$ auf der unendlichen oder auf der endlichen von $Q_{a}$ und $K_{a}$ (auf $q$ ) begrenzten Strecke liegt. Es werden also die beiden Punkte, nämlich die Spur von $g^{(n)}$ auf $q$ und $K^{(n)}$, von $S$ aus durch zwei Strahlen projiziert, welche miteinander einen Winkel $\left[(-2)^{n}-1\right] \boldsymbol{\Omega}_{a}$ bilden; denn dieser Winkel ist gleich $\Omega^{(n)}-\omega^{(n)}-\Omega_{a}$, da, um diesen Winkel zu erhalten, wenn $n$ gerade ist und alsdann $\Omega^{(n)}$ und $\omega^{(n)}$ mit $\Omega$ und $\omega$, also mit $\Omega_{a}$ gleichen Sinn haben, wegen der Abweichung des zweiten Anfangsstrahles $S K_{a}$ rom erstern $S Q_{a}$ um $\Omega_{a}$ von $\left(\Omega^{(n)}-\omega^{(n)}\right)$ der Winkel $\Omega_{a}$ subtrahiert werden muß und, wenn $n$ ungerade ist und alsdann $\Omega^{(n)}$ und $\omega^{(n)}$ zu $\Omega_{a}$ entgegengesetzten Sinn haben, wegen der nämlichen Abweichung zu $\left(\Omega^{(n)}-\omega^{(n)}\right)$ der Winkel $-\Omega_{a}$ addiert werden muß; dieser Winkel ist also in beiden Fällen oder

$$
=(-2)^{n} \Omega-(-2)^{n}\left(\Omega-\Omega_{a}\right)-\Omega_{a}=\left[(-2)^{n}-1\right] \Omega_{a}
$$

B erliner, Habilitationsschrift. 
$=(-2)^{n} \boldsymbol{\Omega}-(-2)^{n}\left(\Omega-\boldsymbol{\Omega}_{a}+\pi\right)-\boldsymbol{\Omega}_{a}=\left[(-2)^{n}-1\right] \boldsymbol{\Omega}_{a}-(-2)^{n} \pi$, wo das Vielfache von $\pi$ weggelassen werden kann.

Durchläuft nun der Strahl $g$ ein Intervall um $P$ und mithin seine Spur $K$ ein Intervall auf $q$, welche beide Intervalle von je zwei aufeinanderfolgenden Elementen einer Gruppe in $(P)^{2^{n}}$ bzw. in $(q)^{2^{n}}$ begrenzt werden und mehr kein Element derselben Gruppe enthalten, so durchlaufen die $n$-ten Repräsentanten $g^{(n)}$ von $g$ und $K^{(n)}$ von $K$ solche Intervalle, welche von je zwei aufeinanderfolgenden Elementen einer Gruppe in $(P)^{2^{n-n}}=(P)^{1}$ bzw. in $(q)^{2^{n-n}}=(q)^{1}$ begrenzt werden und mehr kein Element derselben Gruppe enthalten (nach Nr. 26 mit Hilfe des Schlusses von $n$ auf $n+1$ oder wie aus Satz 15 leicht zu entnehmen ist), also den ganzen Büschel um $P$ bzw. die ganze Gerade $q$ einmal. Die beiden, während dieser Bewegungen, von $K^{(n)}$ und der Spur von $g^{(n)}$ auf $q$ beschriebenen Punktreihen, welche, weil diese Spur und $K^{(n)}$ von $S$ aus durch zwei einen konstanten Winkel, nämlich den Winkel $\left[(-2)^{n}-1\right] \Omega_{a}$, bildende Strahlen projiziert werden, von $S$ aus durch zwei konzentrische, gleiche und gleichlaufende Strahlenbüschel projiziert werden, müssen projektiv sein für jeden beliebigen, aber positiven ganzen Wert von $n$. Diese beiden projektiven Punktreihen sind n ur dann identisch, wenn der konstante Winkel $\left[(-2)^{n}-1\right] \boldsymbol{\Omega}_{a}= \pm k \pi,(-1)^{n}\left[2^{n}-(-1)^{n}\right] \boldsymbol{\Omega}_{a}$ $= \pm k \pi,\left[2^{n}-(-1)^{n}\right]\left|\Omega_{\alpha}\right|=k \pi$, also $\left|\Omega_{a}\right|=\frac{k \pi}{2^{n}-(-1)^{n}}$ (wo $k$ eine beliebige ganze positive Zahl bedeutet), wenn also (s. weiter unten Satz 19 in Nr.29) $p_{A}$ durch einen solchen Punkt von $q$ geht, der ein $n$-ter Koinzidenz-Repräsentant ist. Sollen diese projektiven Punktreihen auf $q$ für jeden beliebigen positiven ganzen Wert von $n$ identisch sein, so wird dann, weil nur die Zahl 3 in $\left[2^{n}-(-1)^{n}\right]$ für jeden beliebigen positiven ganzen Wert von $n$ aufgeht, $\left|\boldsymbol{\Omega}_{a}\right|=\frac{l \pi}{3}$ sein müssen, wo $l=0,1$ oder 2 (da sonst $\left[(-2)^{n}-1\right] \Omega_{a}= \pm k \pi$ nicht für jeden beliebigen Wert von $n$ sein konnte), also $p_{A}$ durch $Q_{a} \equiv q a, Q_{b} \equiv q b$ oder $Q_{c} \equiv q c$ gehen (nach Nr.4) und somit $q$ durch $p_{A} a, p_{A} b$ oder $p_{A} c$; nun trifft aber $p_{A}$ die Dreieckseite $a$, die auch Tangente an $P^{2}$ ist, in deren Berührungspunkt (Nr. 2) und die Dreieckseiten $b$ und $c$, die gleichfalls Tangenten an $P^{2}$ sind, in deren Schnittpunkt $A$; es wird also dann $q$ keine andere Tangente von $P^{2}$ als eine der Dreieck- 
seiten $a, b, c$ sein können. Die nämlichen beiden projektiven Punktreihen auf $q$ liegen ferner nur dann involutorisch, wenn der konstante Winkel $\left[(-2)^{n}-1\right] \boldsymbol{\Omega}_{a}= \pm \frac{2 k+1}{2} \pi$, also $\left|\boldsymbol{\Omega}_{a}\right|$ $=\frac{(2 k+1) \pi}{2\left[2^{n}-(-1)^{n}\right]}($ wo $k$ eine beliebige positive ganze Zahl bedeutet), wenn also (s. weiter unten Nr.42) $p_{A}$ durch einen solchen Punkt von $q$ geht, dessen erster Repräsentant ein $n$-ter Koinzidenz-Repräsentant ist. Sollen diese beiden projektiven Punktreihen auf $q$ für jeden beliebigen positiven ganzen Wert von $n$ involutorische Lage haben, so wird dann $\left|\Omega_{a}\right|=\frac{l \pi}{2.3}=\frac{l \pi}{6}$ sein müssen, wo $l=1,3$ oder 5 (da sonst $\left[(-2)^{n}-1\right] \Omega_{a}= \pm \frac{2 k+1}{2} \pi$ nicht für jeden beliebigen Wert von $n$ sein konnte), und also $p_{A}$ durch $Q_{b}^{\prime}, Q_{a}^{\prime}$ oder $Q_{c}^{\prime}$ gehen (nach Anmerkung in $\mathrm{Nr} .22$ ), wo die auf der Tangente $q$ von $P^{2}$ liegenden Punkte $Q_{a}^{\prime}, Q_{b}^{\prime}, Q_{c}^{\prime}$ die vierten harmonischen zu je einem der drei Punkte $Q_{a}, Q_{b}, Q_{c}$ in bezug auf die beiden andern sind und wo also durch $Q_{a}^{\prime}, Q_{b}^{\prime}, Q_{c}^{\prime}$ der Reihe nach diejenigen Tangenten $p_{\alpha}^{\prime}, p_{b}^{\prime}, p_{c}^{\prime}$ von $P^{2}$ gehen, welche an $P^{2}$ die vierten harmonischen Tangenten zu je einer der Dreieckseiten $a, b, c$ (welche Seiten gleichfalls Tangenten an $P^{2}$ sind) in bezug auf die beiden andern sind; es wird somit dann $q$ durch einen der drei Schnittpunkte von $p_{A}$ mit $p_{a}^{\prime}, p_{b}^{\prime}, p_{c}^{\prime}$ gehen müssen. Nun sind aber $a, p_{a}^{\prime}, p_{b}^{\prime}$ und $p_{c}^{\prime}$ die Polaren der Punkte $P_{a}, P_{a}^{\prime}, P_{b}^{\prime}$ und $P_{c}^{\prime}$ (Nr.23), also bilden $a$ und $p_{a}^{\prime}$ ein Paar in der induzierten Tangenteninvolution $\left(P^{2}\right)^{2}$, deren Zentrum $P$ ist (Nr. 7), und es sind $a p_{a}^{\prime} p_{b}^{\prime} p_{c}^{\prime}$ vier harmonische Tangenten an $P^{2}$ (nach Nr.4); folglich muß die Ecktransversale $p_{A}$, die durch das Involutionszentrum $P$ von $\left(P^{2}\right)^{2}$ und durch den Berührungspunkt der Tangente $a$ von $P^{2}$ geht (Nr. 2), auch durch den Berührungspunkt der zu $a$ in $\left(P^{2}\right)^{2}$ zugepaarten Tangente $p_{a}^{\prime}$ gehen und mithin auch durch den Schnittpunkt von $p_{b}^{\prime}$ und $p_{c}^{\prime}$, da nun $p_{A}$, die die Berührungspunkte von $a$ und $p_{a}^{\prime}$ verbindende Gerade, durch den Pol derjenigen Geraden in bezug auf $P^{2}$ gehen muß, welche die beiden Berührungspunkte der durch $a$ und $p_{a}^{\prime}$ harmonisch getrennten Tangenten $p_{b}^{\prime}$ und $p_{c}^{\prime}$ verbindet. Wenn also jene beiden projektiven Punktreihen auf $q$ für jeden beliebigen positiven ganzen Wert von $n$ involutorische Lage haben sollen, so 
wird dann $q$, die durch $p_{A} p_{u}^{\prime}, p_{A} p_{b}^{\prime}$ oder $p_{A} p_{c}^{\prime}$ gehen muß, keine andere Tangente von $P^{2}$ als eine der drei: $p_{a}^{\prime}, p_{b}^{\prime}, p_{c}^{\prime}$ sein können.

Die für jene beiden Punktreihen auf $q$, nämlich für die von $K^{(n)}$ und die von der Spur von $g^{(n)}$ beschriebenen, gefundenen Resultate gelten nun auch, wenn an Stelle der letztern Punktreihe der von $g^{(n)}$ um $P$ beschriebene Strahlenbüschel tritt.

Nun muß aber diejenige Gruppe in $(P)^{2^{n}}$ bzw. in $(q)^{2^{n}}$, der der Strahl $g$ bzw. dessen Spur $K$ auf $q$ angehört, die ganze Involution $(P)^{2^{n}}$ bzw. $(q)^{2^{n}}$ einmal durchlaufen, wenn $g$ und mithin $K$ solche Intervalle durchlaufen, welche von je zwei aufeinanderfolgenden Elementen einer Gruppe in $(P)^{2^{n}}$ bzw. in $(q)^{2^{n}}$ begrenzt werden und mehr kein Element derselben Gruppe enthalten; da zwischen zwei aufeinanderfolgenden Elementen einer Gruppe in $(P)^{2^{n}}$ bzw. in $(q)^{2^{n}}$ nur ein Element jeder andern Gruppe von $(P)^{2^{n}}$ bzw. von $(q)^{2^{n}}$ liegt (Nr.22). Ferner sind die $n$-ten Repräsentanten $g^{(n)}$ von $g$ und $K^{(n)}$ von $K$ zugleich die Repräsentanten derjenigen Gruppen in $(P)^{2^{n}}$ und $(q)^{2^{n}}$, den $g$ bzw. $K$ angehören (Nr.24). Mithin können wir den gefundenen Resultaten auch die folgende Form geben:

$\mathrm{Satz}$ 16. Derjenige Strahlenbüschel um einen Punkt $P$, welcher von den Repräsentanten der Strahlengruppen in $(P)^{2^{n}}$ gebildet wird, und diejenige Punktreihe auf irgendeiner Tangente $q$ des Polarkegelschnitts $P^{2}$ von $p$ (der Polare von $P$ ), welche von den Repräsentanten der Punktgruppen in $(q)^{2^{n}}$
Diejenige Punktreihe auf einer Geraden $p$, welche von den Repräsentanten der Punktgruppen in $(p)^{2^{n}}$ gebildet wird, und derjenige Strahlenbüschel um irgendeinen Punkt $G$ des Polarkegelschnitts $p^{2}$ von $P$ (dem Pole von $p$ ), welcher von den Repräsentanten der Strahlengruppen in $(G)^{2^{n}}$

gebildet wird, sind für jeden beliebigen positiven ganzen Wert von $n$ projektiv mit solchen Elementen als entsprechenden, welche Repräsentanten inzidenter Gruppen

$$
\text { in }(P)^{2^{n}} \text { und }(q)^{2^{n}} \text { sind. } \mid \quad \text { in }(p)^{2^{n}} \text { und }(G)^{2^{n}} \text { sind. }
$$

Diese beiden projektiven Gebilde haben nur dann perspektive bzw. involutorische Lage, wenn einer und mithin jeder (s. weiter unten $\mathrm{Nr} .33$ und 34) der drei 
Strahlen, welche die durch $P$ gehenden Ecktransversalen von $A B C$ sind, durch einen solchen Punkt von $q$ geht,

Punkte, in denen $p$ von den Seiten von $A B C$ geschnitten wird, auf einem solchen Strahle von $G$ liegt,

welcher selbst ein $n$-ter Koinzidenz-Repräsentant ist bzw. dessen erster Repräsentant ein $n$-ter Koinzidenz-Repräsentant ist.

Ist $q$ von den Seiten von $A B C$ bzw. von den drei Tangenten $p_{a}^{\prime}, p_{b}^{\prime}, p_{c}^{\prime}\left(\operatorname{von} P^{2}\right)$

Ist $G$ von den Ecken von $A B C$ bzw. von den drei Punkten $P_{A}^{\prime}, P_{B}^{\prime}, P_{C}^{\prime}\left(\operatorname{von} p^{2}\right)$ verschieden, so können jene beiden projektiven Gebilde nicht für jeden beliebigen positiven ganzen Wert von $n$ perspektive bzw. involutorische Lage haben.

Zugleich ist der Satz 14 in Nr. 23 nochmals bewiesen worden.

28. Aus dem Satze 15 ergibt sich ferner:

Satz 17. Jedes Paar der von $A B C$ im Gebilde erster Stufe erzeugten Involution zweiten Grades wird durch seinen Repräsentanten, der der erste Repräsentant eines jeden der beiden Elemente des Paares ist, und den zweiten Repräsentanten, der gleichfalls für beide Elemente des Paares der nämliche ist, harmonisch getrennt.

Bilden nämlich die Punkte $Q$ und $Q^{\prime}$ ein Paar in der von $A B C$ auf der Geraden $p$ erzeugten Involution $(p)^{2}$ und ist $Q^{(1)}$ der erste Repräsentant von $Q$ und $Q^{\prime}, Q^{(2)}$ der zweite Repräsentant derselben und $\omega$ der Ordinatenwinkel von $Q$, so ist (nach Satz 15) $-2 \omega$ der Ordinatenwinkel von $Q^{(1)}$ und $+4 \omega$ der von $Q^{(2)}$. Es ist also der Winkel $Q R Q^{(1)}$ (wo $R$ derjenige Punkt ist, aus dem $(p)^{2}$ durch eine rechtwinklige Strahleninvolution projiziert wird) gleich $-2 \omega-\omega=-3 \omega$ und der Winkel $Q R Q^{(2)}$ gleich $4 \omega-\omega=+3 \omega$, ferner ist $Q R Q^{\prime}= \pm \frac{\pi}{2} ;$ mithin sind $R Q$ und $R Q^{\prime}$ die Halbierungsgeraden des Winkels $Q^{(1)} R Q^{(2)}$ und $Q Q^{\prime} Q^{(1)} Q^{(2)}$ müssen vier harmonische Punkte sein.

Aus dem Satze 17 folgt nun:

Satz 18. Ist $g$ irgendeine durch den Punkt $P$
Ist $Q$ irgendein auf der Gerade $p$ liegender Punkt 
gehende Gerade und $p^{2}$ der Polarkegelschnitt von $P$ und verbindet man den $\mathrm{Pol} G$ von $g$ in bezug auf $A B C$ mit dem Pole von $g$ in bezug auf $p^{2}$ durch eine Gerade, so wird diese Gerade von $p^{2}$ zum zweitenmal im Pole $G^{(1)}$ des ersten Repräsentanten $g^{(1)}(\equiv P G)$ von $g$ geschnitten. und $P^{2}$ der Polarkegelschnitt von $p$ und bringt man die Polare $q$ von $Q$ in bezug auf $A B C$ mit der Polare von $Q$ in bezug auf $P^{2}$ zum Schnitt, so ist die zweite von diesem Schnittpunkt an $P^{2}$ gehende Tangente die Polare $q^{(1)}$ des ersten Repräsentanten $Q^{(1)}$ $(\equiv p q)$ von $Q$.

Denn ist $g_{1} g_{1}^{\prime}$ dasjenige Strahlenpaar in der von $A B C$ erzeugten Involution $(P)^{2}$, dessen Pole $G_{1}$ und $G_{1}^{\prime}$ (in bezug auf $A B C)$ die Schnittpunkte der durch $P$ gehenden Geraden $g$ mit $p^{2}$ sind und dessen Repräsentant also $g$ ist, so sind (nach Satz 17) $g_{1} g_{1}^{\prime} g\left(P G \equiv g^{(1)}\right)$ vier harmonische Strahlen um $P$ und mithin, weil der Büschel der Polaren um $P$ zu der Punktreihe der Pole auf $p^{2}$ projektiv ist, ihre Pole $G_{1} G_{1}^{\prime} G G^{(1)}$ vier harmonische Punkte auf $p^{2}$. Folglich muß die $G$ mit $G^{(1)}$ verbindende Gerade zu der $G_{1}$ mit $G_{1}^{\prime}$ verbindenden Geraden $g$ konjugiert sein in bezug auf $p^{2}$ und mithin durch den Pol von $g$ in bezug auf $p^{2}$ gehen.

29. Kehren wir nun zu der in Nr.24 aufgeworfenen Frage zurück.

Soll ein Element $Q$, dessen Ordinatenwinkel $\omega$ ist, wo $0<\omega \leq \pi$, mit seinem $n$-ten Repräsentanten $Q^{(n)}$, dessen Ordinatenwinkel $\omega^{(n)}$ ist, zusammenfallen, so wird dann (nach der Definition der Ordinatenwinkel), wenn $n=2 m-1 \quad(m \geqq 1)$ und also $Q$ und $Q^{(n)}$ sich im Gebilde entgegengesetzt bewegen,

$$
\left|\omega^{(2 m-1)}\right|-k_{1} \pi=\pi-\omega
$$

sein müssen, wo $k_{1}$ die Anzahl der vollen Umläufe von $Q^{(n)}$ im Gebilde angibt und wo $k_{1}$ auch $=0$ sein kann, also:

$$
\omega^{(2 m-1)}=-\left(\pi-\omega+k_{1} \pi\right)=-\left[\left(k_{1}+1\right) \pi-\omega\right]
$$

und, wenn $n=2 m$ und also $Q$ und $Q^{(n)}$ sich im Gebilde im gleichen Sinne bewegen,

$$
\omega^{(2 m)}-k_{2} \pi=\omega,
$$


wo $k_{2}$ dieselbe Bedeutung wie $k_{1}$ hat, wo aber (weil die Bewegung von $Q^{(n)}$ nach Satz 15 mit einer größeren Geschwindigkeit als die von $Q$ sich vollzieht) $k_{2} \geqq 1$ sein muß, also:

$$
\omega^{(2 m)}=k_{2} \pi+\omega \text {. }
$$

Nach Satz 15 ist aber:

$$
\omega^{(n)}=(-2)^{n} \omega,
$$

mithin muß, wenn anstatt $\left(k_{1}+1\right)$ und $k_{2}$, welche letzte $\geqq 1$ sein muß, $k$ gesetzt wird,

$$
\omega^{(2 m-1)}=-(k \pi-\omega)=-2^{2 m-1} \cdot \omega
$$

sein, also:

und

$$
\omega=\frac{k \pi}{2^{2 m-1}+1}, \quad(k \geqq 1),
$$

also

$$
\omega^{(2 m)}=k \pi+\omega=2^{2 m} \cdot \omega,
$$

$$
\omega=\frac{k \pi}{2^{2 m}-1}, \quad(k \geqq 1) .
$$

Hat, umgekehrt, der Ordinatenwinkel $\omega$ eines Elementes $Q$ die Form $\frac{k \pi}{2^{2 m-1}+1}$ oder $\frac{k \pi}{2^{2 m}-1}$, wo $k$ und $m$ beliebige ganze Zahlen $(\geqq 1)$ bedeuten, so muß $Q$ mit $Q^{(2 m-1)}$ bzw. mit $Q^{(2 m)}$ zusammenfallen, da dann:

$$
\begin{gathered}
\boldsymbol{\omega}^{(2 m-1)}=-2^{2 m-1} \cdot \boldsymbol{\omega}=-\frac{2^{2 m-1} \cdot k \pi}{2^{2 m-1}+1}=-\left(k \pi-\frac{k \pi}{2^{2 m-1}+1}\right) \\
=-[(k-1) \pi+(\pi-\omega)]
\end{gathered}
$$

bzw.

$$
\omega^{(2 m)}=2^{2 m} \cdot \omega=\frac{2^{2 m} \cdot k \pi}{2^{2 m}-1}=k \pi+\frac{k \pi}{2^{2 m}-1}=k \pi+\omega
$$

und also $\omega$ und $\omega^{(2 m-1)}$ bzw. $\omega^{(2 m)}$ die Ordinatenwinkel eines und desselben Elementes, das nur auf verschiedenen Wegen in seine Lage gelangte, sind.

Weil aber zwei Elemente, deren Ordinatenwinkel sich nur um ein Vielfaches von $\pi$ voneinander unterscheiden, zusammenfallen, so gibt es nur $2^{2 m-1}+1$ bzw. $2^{2 m}-1$ voneinander verschiedene Elemente, deren Ordinatenwinkel die Form $\frac{k \pi}{2^{2 m-1}+1}$ bzw. $\frac{k \pi}{2^{2 m}-1}$ haben, nämlich $k=1,2,3, \ldots 2^{2 m-1}+1$ bzw. $k=1,2,3, \ldots 2^{2 m}-1$; diese und nur diese $2^{2 m-1}+1$ bzw. 
$2^{2 m}-1$ Elemente fallen mit ihren $\left(2^{2 m-1}\right)$-ten bzw. $\left(2^{2 m}\right)$-ten Repräsentanten zusammen.

Hiermit ist die aufgeworfene Frage vollständig beantwortet und wir haben, indem wir ein Element, das mit seinem $n$-ten Repräsentanten zusammenfällt, einen $n$-ten Koinzidenz-Repräsentanten nennen:

Satz 19. Es gibt in einem Gebilde erster Stufe nur $2^{n}-(-1)^{n} n$-te Koinzidenz-Repräsentanten, wo $n$ irgendeine positive ganze Zahl bedeutet. Die notwendige und hinreichende Bedingung dafür, daß ein Element ein $n$-ter KoinzidenzRepräsentant sei, ist, daß der Ordinatenwinkel des Elementes auf die Form $\frac{k \pi}{2^{n}-(-1)^{n}}$ gebracht werden kann, wo $k$ irgendeine ganze Zahl, die Null nicht ausgeschlossen, bedeutet.

Die $n$-ten Koinzidenz-Repräsentanten sind im Gebilde in der Weise verteilt, daß zwischen je zwei aufeinanderfolgenden Elementen einer jeden Gruppe in der von $A B C$ im Gebilde erzeugten Involution $\left(2^{n}\right)$-ten Grades nur ein $n$-ter Koinzidenz-Repräsentant liegt; eine Ausnahme hiervon machen nur diejenigen beiden aufeinanderfolgenden Elemente der Gruppe, zwischen denen der Repräsentant derselben Gruppe (welcher Repräsentant der $n$-te Repräsentant eines jeden der $2^{n}$ Elemente der Gruppe ist) liegt, indem zwischen diesen beiden Elementen entweder zwei $n$-te Koinzidenz-Repräsentanten liegen, und zwar der eine vor und der andere nach dem Repräsentanten der Gruppe, oder kein einziger, je nachdem $n$ ungerade oder gerade ist.

Das letzte ergibt sich folgendermaßen. Die Differenz der Ordinatenwinkel zweier aufeinanderfolgender Elemente $Q_{n, i}$ und $Q_{n, i+1}$ der Gruppe bzw. zweier aufeinanderfolgender $n$-ter Koinzidenz-Repräsentanten ist (absolut genommen) $\frac{\pi}{2^{n}}$ bzw. $\frac{\pi}{2^{n}-(-1)^{n}}$. Also muß, wenn $n$ ungerade ist, zwischen $Q_{n, i}$ und $Q_{n, i+1}$ (für jeden Wert von $i=1,2, \ldots 2^{n}$ ) ein $n$-ter Koinzidenz-Repräsentant liegen. Sollen aber zwischen $Q_{n, i}$ und $Q_{n, i+1}$ (für einen Wert von $i$ ) zwei $n$-te Koinzidenz-Repräsentanten liegen, so wird, während ein Element $Q$ das Intervall zwischen $Q_{n, i}$ und $Q_{n, i+1}$ und mithin sein $n$-ter Repräsentant $Q^{(n)}$ im entgegengesetzten Sinne, 
vom Repräsentanten der Gruppe ausgehend und nach demselben zurückkehrend, das ganze Gebilde einmal durchlaufen werden, $Q$ mit $Q^{(n)}$ zweimal, nämlich in den beiden zwischen $Q_{n, i}$ und $Q_{n, i+1}$ liegenden $n$-ten Koinzidenz-Repräsentanten zusammenfallen müssen; dies wird aber nur dann eintreten, wie man leicht einsieht, wenn zwischen $Q_{n, i}$ und $Q_{n, i+1}$ der Repräsentant der Gruppe liegt, und alsdann muß derselbe Repräsentant auch zwischen beiden $n$-ten Koinzidenz-Repräsentanten liegen. Ferner kann, wenn $n$ gerade ist und dann $\frac{\pi}{2^{n}-(-1)^{n}}=\frac{\pi}{2^{n}-1}$, zwischen $Q_{n, i}$ und $Q_{n, i+1}$ mehr als ein $n$-ter Koinzidenz-Repräsentant nicht liegen. Soll aber zwischen $Q_{n, i}$ und $Q_{n, i+1}$ (für irgendeinen Wert von $i)$ kein einziger $n$-ter Koinzidenz-Repräsentant liegen, so wird, während $Q$ das Intervall zwischen $Q_{n, i}$ und $Q_{n, i+1}$ und mithin $Q^{(n)}$ im selben Sinne, vom Repräsentanten der Gruppe ausgehend und nach demselben zurïckkehrend, das ganze Gebilde einmal durchlaufen werden, $Q$ mit $Q^{(n)}$ nirgends zusammenfallen; dies wird aber nur dann eintreten, wenn zwischen $Q_{n, i}$ und $Q_{n, i+1}$ der Repräsentant der Gruppe liegt.

30. Wie wir sahen (Nr. 24) ist der $n$-te Repräsentant $Q^{(n)}$ eines Elementes $Q$ eindeutig bestimmt und der $(i+k)$-te Repräsentant $Q^{(i+k)}$ von $Q$ ist zugleich der $k$-te Repräsentant $Q^{(i)}(k)$ des $i$-ten Repräsentanten $Q^{(i)}$ von $Q$ für jeden beliebigen Wert von $i$ und von $k$. Ist nun ein Element $Q$ ein $k$-ter KoinzidenzRepräsentant, ist also $Q^{(k)} \equiv Q$, so muß auch $Q^{(i)(k)} \equiv Q^{(i+k)} \equiv Q^{(k)(i)}$ $\equiv Q^{(i)}$ und $Q^{(m k)} \equiv Q^{[k+(m-1) k]} \equiv Q^{(k)[(m-1) k]} \equiv Q^{[(m-1) k]} \equiv Q^{[(m-2) k]}$ $\equiv \ldots \equiv Q^{(k)} \equiv Q$ sein, d. h. es muß dann auch der $i$-te Repräsentant $Q^{(i)}$ von $Q$ für jeden beliebigen Wert von $i$ ein $k$-ter Koinzidenz-Repräsentant sein und $Q$ und mithin auch alle seine Repräsentanten müssen zugleich $n$-te Koinzidenz-Repräsentanten sein, wenn $n$ irgendein Vielfaches von $k$ ist. (Diese Resultate sind auch aus der Form $\frac{7 \pi}{2^{k}-(-1)^{k}}$ des Ordinatenwinkels eines $k$-ten Koinzidenz-Repräsentanten mit Hilfe der Sätze 15 und 19 zu entnehmen.) Jeder $n$-te Koinzidenz-Repräsentant, welcher kein früherer Koinzidenz-Repräsentant ist, wo also das Element erst mit seinem $n$-ten (und mit keinem frühern) Repräsentanten zusammenfällt, soll ein primitiver $n$-ter Koinzidenz-Repräsentant heißen. 
Ist $Q$ ein primitiver $n$-ter Koinzidenz-Repräsentant, so sind auch seine Repräsentanten $Q^{(1)}, Q^{(2)}, Q^{(3)}, \ldots Q^{(n-1)}$ primitive $n$-te Koinzidenz-Repräsentanten. Denn diese müssen, wie soeben gesehen, $n$-te Koinzidenz-Repräsentanten sein, wäre irgendeiner unter ihnen, etwa $Q^{(i)}$, schon ein $k$-ter $(k<n)$, so müßte, gegen die Voraussetzung, $Q$ ebenso wie $Q^{(i)}$ schon ein $k$-ter und also kein primitiver $n$-ter sein; da $Q^{(n)} \equiv Q$ und also $Q$ ein $(n-i)$-ter Repräsentant von $Q^{(i)}$ ist. Jeder weitere Repräsentant $Q^{(m)}$ von $Q$ $(m \geqq n)$ muß mit einem der $n$ Elemente $Q, Q^{(1)}, Q^{(2)}, \ldots Q^{(n-1)}$ zusammenfallen und zwar, wenn $m \equiv i(\bmod n)$, mit $Q^{(i)}$ (wo $i<n$, ist $i=0$, so soll unter $Q^{(0)}$, dem nullten Repräsentanten von $Q$, das Element $Q$ selbst verstanden werden). Die $n$ primitiven $n$-ten Koinzidenz-Repräsentanten aber sind sämtlich voneinander verschieden. Denn fiele etwa $Q^{(p)}$ mit $Q^{(q)}$ zusammen $(q<p<n)$, so wäre, gegen das soeben Bewiesene, $Q^{(q)}$ schon ein $(p-q)$-ter-und also kein primitiver $n$-ter Koinzidenz-Repräsentant.

Es bildet also das Element $Q$, das ein primitiver $n$-ter Koinzidenz-Repräsentant ist, zusammen mit seinen $n-1$ Repräsentanten $Q^{(1)}, Q^{(2)}, \ldots Q^{(n-1)}$ eine geschlossene Gruppe von $n$ Elementen, primitiven $n$-ten Koinzidenz-Repräsentanten, welche Gruppe die Eigenschaft besitzt, daß von je zweien ihrer Elemente das eine etwa der $k$-te Repräsentant $(k<n)$ des andern und dieses letztere der $(n-k)$-te (und kein früherer) Repräsentant des erstern ist (denn der $(n-k)$-te Repräsentant des erstern Elementes, welcher Repräsentant zugleich der $(n-k+k=n)$-te Repäsentant des letztern Elementes ist, muß mit diesem letztern zusammenfallen); man gelangt also auch dann noch zu der ganzen Gruppe, wenn man anstatt von $Q$ von irgendeinem andern Elemente der Gruppe ausgeht.

Jeder weitere (der Gruppe $Q Q^{(1)} Q^{(2)} \ldots Q^{(n-1)}$ nicht angehörende) primitive $n$-te Koinzidenz-Repräsentant $R$ des Gebildes führt zu einer ebenso beschaffenen Gruppe von $n$ voneinander verschiedenen primitiven $n$-ten Koinzidenz-Repräsentanten $R R^{(1)} R^{(2)} \ldots R^{(n-1)}$; kein Element dieser Gruppe fällt mit einem der Gruppe $Q Q^{(1)} \ldots Q^{(n-1)}$ zusammen. Denn wäre etwa $R^{(i)} \equiv Q^{(k)}$ $(i<n, k<n)$, so müßte $R \equiv R^{(n)} \equiv R^{(i)(n-i)} \equiv Q^{(k)(n-i)} \equiv Q^{(n+k-i)} \equiv Q^{(r)}$ sein, wo $r<n$ und $r \equiv n+k-i(\bmod . n)$, und also $R$ gegen die Voraussetzung in der Gruppe $Q Q^{(1)} \ldots Q^{(n-1)}$ vorkommen. 
Die primitiven $n$-ten Koinzidenz-Repräsentanten eines Gebildes erster Stufe zerfallen also in Gruppen von je $n$ Elementen, wobei jede Gruppe aus irgendeinem ihr angehörenden primitiven $n$-ten Koinzidenz-Repräsentanten und dessen $n-1$ niedrigsten Repräsentanten besteht. Jeder primitive $n$-te Koinzidenz-Repräsentant gehört einer und nur einer solchen Gruppe an.

31. Ist ein Element $Q$ ein primitiver $i$-ter und zugleich ein $n$-ter Koinzidenz-Repräsentant, so muß $i$ ein Teiler von $n$ sein; denn wäre $n=q i+r$, wo $0<r<i$, so müßte, weil $Q^{(i)} \equiv Q$ und $Q^{(n)} \equiv Q, Q^{(r)} \equiv Q^{(q i)(r)} \equiv Q^{(q i+r)} \equiv Q^{(n)} \equiv Q$ und also $Q$ schon ein $r$-ter und kein primitiver $i$-ter Koinzidenz-Repräsentant sein. Ist ferner $Q$ ein $n$-ter und zugleich ein $m$-ter Koinzidenz-Repräsentant, so muß $Q$ auch ein $d$-ter Koinzidenz-Repräsentant sein, wo $d$ der größte gemeinsame Teiler von $n$ und $m$ ist; denn ist $Q$ etwa ein primitiver $i$-ter Koinzidenz-Repräsentant, so muß $i$, wie soeben gesehen, ein Teiler von $n$ und ebenso von $m$ und also auch von $d$ sein, mithin muß auch $Q^{(d)} \equiv Q$ sein $(\mathrm{Nr} .30)$.

Hiervon wollen wir Gebrauch machen zur Bestimmung der Anzahl der primitiven $n$-ten Koinzidenz-Repräsentanten.

$\mathrm{Zu}$ diesem Ende ist notwendig und hinreichend, aus dem Komplex der $n$-ten Koinzidenz-Repräsentanten die $\left(\frac{n}{p_{1}}\right)$-ten, $\left(\frac{n}{p_{2}}\right)$-ten, $\left(\frac{n}{p_{3}}\right)$-ten, $\ldots$ und $\left(\frac{n}{p_{k}}\right)$-ten Koinzidenz-Repräsentanten auszuscheiden, wo $p_{1}<p_{2}<p_{3}<\cdots<p_{k}$ die $k$ voneinander verschiedenen Primfaktoren von $n$ sind, wo also $n=p_{1}^{\pi_{1}} p_{2}^{\pi_{2}} p_{3}^{\pi_{3}} \ldots p_{k}^{\pi_{k}}$; die sodann überbleibenden werden sämtlich primitive $n$-te Koinzidenz-Repräsentanten sein. Denn ein $\left(\frac{n}{p_{i}}\right)$-ter Koinzidenz-Repräsentant (wo $p_{i}$ irgendeiner der Primteiler von $n$ ist) ist zugleich ein nicht primitiver $n$-ter, und jeder nicht primitive $n$-te muß ein primitiver $f$-ter, wo $f<n$ und $f$ ein Teiler von $n$ und also auch von $\frac{n}{p_{i}}$ ist (wo $p_{i}$ mindestens einer der Primteiler von $n$ sein muß), und mithin auch ein $\left(\frac{n}{p_{i}}\right)$-ter Koinzidenz-Repräsentant sein. 
Wir scheiden nun aus dem Komplex der $n$-ten KoinzidenzRepräsentanten, deren Anzahl $2^{n}-(-1)^{n}$ beträgt, zuerst die $\left(\frac{n}{p_{1}}\right)$-ten, deren Anzahl $2^{n: p_{1}}-(-1)^{n: p_{1}}$ beträgt, aus; alsdann bleibt ein zweiter Komplex übrig, der nur noch diejenigen $n$-ten Koinzidenz-Repräsentanten enthält, welche mehr keine $\left(\frac{n}{p_{1}}\right)$-te sind und deren Anzahl

1)

$$
2^{n}-2^{n: p_{1}}-(-1)^{n}+(-1)^{n: p_{1}}
$$

beträgt. Von den $2^{n: p_{2}}-(-1)^{n: p_{2}}\left(\frac{n}{p_{2}}\right)$-ten Koinzidenz-Repräsentanten, die im ersten Komplex vorhanden waren, sind im zweiten Komplex nur noch $2^{n: p_{2}}-2^{n: p_{2} p_{1}}-(-1)^{n: p_{2}}+(-1)^{n: p_{2} p_{1}}$ $=2^{n: p_{2}}-2^{n: p_{2} p_{1}}-(-1)^{n}+(-1)^{n: p_{1}} \quad\left(\right.$ da $p_{2}>p_{1}$, also $p_{2}$ sicher ungerade und $\frac{n}{p_{2}}$ zugleich mit $n$ und $\frac{n}{p_{1} p_{2}}$ zugleich mit $\frac{n}{p_{1}}$ gerade oder ungerade ist) geblieben. Denn, wie wir sahen, ist jeder $\left(\frac{n}{p_{2}}\right)$-te, der zugleich ein $\left(\frac{n}{p_{1}}\right)$-ter ist, auch ein $\left(\frac{n}{p_{1} p_{2}}\right)$-ter und, umgekehrt, ist jeder $\left(\frac{n}{p_{1} p_{2}}\right)$-te zugleich ein $\left(\frac{n}{p_{1}}\right)$-ter und ein $\left(\frac{n}{p_{2}}\right)$-ter; mithin ist für die $\left(\frac{n}{p_{2}}\right)$-ten Koinzidenz-Repräsentanten die Ausscheidung der $\left(\frac{n}{p_{1}}\right)$-ten mit der Ausscheidung der $\left(\frac{n}{p_{2} p_{1}}\right)$-ten gleichbedeutend und wir haben, um die Anzahl der nach der Ausscheidung der $\left(\frac{n}{p_{1}}\right)$-ten übergebliebenen $\left(\frac{n}{p_{2}}\right)$-ten zu bestimmen, in der Formel 1) $\frac{n}{p_{2}}$ statt $n$ zu setzen.

Aus dem zweiten Komplex scheiden wir ferner die $2^{n: p_{2}}$ $-2^{n: p_{1} p_{2}}-(-1)^{n}+(-1)^{n: p_{1}}$ übergebliebenen $\left(\frac{n}{p_{2}}\right)$-ten aus; alsdann bleibt ein dritter Komplex übrig, der nur noch diejenigen $n$-ten Koinzidenz-Repräsentanten enthält, welche weder $\left(\frac{n}{p_{1}}\right)$-te, noch $\left(\frac{n}{p_{2}}\right)$-te sind und deren Anzahl beträgt: 


$$
\begin{aligned}
& 2^{n}-2^{n: p_{1}}-(-1)^{n}+(-1)^{n: p_{1}}-\left[2^{n: p_{2}}-2^{n: p_{1} p_{2}}-(-1)^{n}\right. \\
&\left.+(-1)^{n: p_{1}}\right]= \\
&2)=2^{n}-2^{n: p_{1}}-2^{n: p_{2}}+2^{n: p_{1} p_{2}}=\sum_{\alpha_{1}, \alpha_{2}}(-1)^{\alpha_{1}+\alpha_{2}} 2^{n: p_{1}^{\alpha_{1}}} p_{2}^{\alpha_{2}},
\end{aligned}
$$

wo $\alpha_{1}$ und $\alpha_{2}$ unabhängig voneinander die Werte 0 und 1 annehmen sollen. Von den $2^{n: p_{3}}-(-1)^{n: p_{3}}\left(\frac{n}{p_{3}}\right)$-ten KoinzidenzRepräsentanten, die im ersten Komplex vorhanden waren, sind im dritten Komplex nur noch $2^{n: p_{3}}-2^{n: p_{3} p_{1}}-2^{n: p_{3} p_{2}}+2^{n: p_{3} p_{1} p_{2}}$ geblieben. Denn für die $\left(\frac{n}{p_{3}}\right)$-ten sind die Ausscheidungen der $\left(\frac{n}{p_{1}}\right)$-ten und $\left(\frac{n}{p_{2}}\right)$-ten mit den Ausscheidungen der $\left(\frac{n}{p_{3} p_{1}}\right)$-ten und $\left(\frac{n}{p_{3} p_{2}}\right)$-ten gleichbedeutend und wir haben also in 2) $\frac{n}{p_{3}}$ statt $n \mathrm{zu}$ setzen.

Fahren wir in dieser Weise fort, so bleibt nach der $i$-ten Ausscheidung ein $(i+1)$-ter Komplex übrig, der nur noch diejenigen $n$-ten Koinzidenz-Repräsentanten enthält, welche weder $\left(\frac{n}{p_{1}}\right)$-te, noch $\left(\frac{n}{p_{2}}\right)$-te, ... noch $\left(\frac{n}{p_{i}}\right)$-te sind und deren Anzahl beträgt:

3) $2^{n}-2^{n: p_{1}}-2^{n: p_{2}}-\cdots-2^{n: p_{i}}+2^{n: p_{1} p_{2}}+2^{n: p_{1} p_{3}}+\cdots+2^{n: p_{1} p_{i}}$ $+2^{n: p_{2} p_{3}}+\cdots+2^{n: p_{i-1} p_{i}}-2^{n: p_{1} p_{2} p_{3}}-\cdots-2^{n: p_{i-2} p_{i-1} p_{i}}+$ $2^{n: p_{1} p_{2} p_{3} p_{4}}+\cdots-\cdots+(-1)^{i} 2^{n: p_{1} p_{2} p_{3} \ldots p_{i}}=$

$$
=\sum_{\alpha_{1}, \alpha_{2}, \ldots \alpha_{i}}(-1)^{\alpha_{1}+\alpha_{2}+\cdots+\alpha_{i}} 2^{n: p_{1}^{\alpha_{1}}} p_{2}^{\alpha_{2}} p_{3}^{\alpha_{3}} \ldots p_{i}^{\alpha_{i}}
$$

wo $\alpha_{1}, \alpha_{2}, \alpha_{3}, \ldots \alpha_{i}$ unabhängig voneinander die Werte 0 und 1 annehmen sollen.

Die Richtigkeit hiervon kann durch den Schluß von $n$ auf $n+1$ bewiesen werden. Ist nämlich die Formel 3) für einen bestimmten Wert von $i$ richtig, so muß von den $2^{n: p_{i}+1}$ - $(-1)^{n: p_{i}+1}\left(\frac{n}{p_{i+1}}\right)$-ten Koinzidenz-Repräsentanten, die im ersten Komplex vorhanden waren, im $(i+1)$-ten Komplex nur noch $\sum_{\alpha_{1}, \alpha_{2}, \ldots \alpha i}(-1)^{\alpha_{1}+\alpha_{2}+\cdots+\alpha_{i}} 2^{n: p_{i}+1} p_{1}^{\alpha_{1}} p_{2}^{\alpha_{2}} \cdots p_{i}^{\alpha_{i}}$ geblieben sein; da 
für die $\left(\frac{n}{p_{i+1}}\right)$-ten die Ausscheidungen der $\left(\frac{n}{p_{1}}\right)$-ten, $\left(\frac{n}{p_{2}}\right)$-ten, $\ldots$ und $\left(\frac{n}{p_{i}}\right)$-ten mit den Ausscheidungen der $\left(\frac{n}{p_{i+1} p_{1}}\right)$-ten, $\left(\frac{n}{p_{i+1} p_{2}}\right)$-ten, $\ldots$ und $\left(\frac{n}{p_{i+1} p_{i}}\right)$-ten gleichbedeutend sind und also in 3) $\frac{n}{p_{i+1}}$ statt $n \mathrm{zu}$ setzen ist. Mithin bleibt nach der $(i+1)$-ten Ausscheidung ein $(i+2)$-ter Komplex übrig, der nur noch diejenigen $n$-ten Koinzidenz-Repräsentanten enthält, welche weder $\left(\frac{n}{p_{1}}\right)$-te, noch $\left(\frac{n}{p_{2}}\right)$-te, $\ldots$ noch $\left(\frac{n}{p_{i}}\right)$-te, noch $\left(\frac{n}{p_{i+1}}\right)$-te sind und deren Anzahl beträgt:

$$
\begin{aligned}
& \sum_{\alpha_{1}, \alpha_{2}, \ldots \alpha_{i}}(-1)^{\alpha_{1}+\alpha_{2}+\cdots+\alpha_{i}} 2^{n: p_{1}^{\alpha_{1}} p_{2}^{\alpha_{2}} \ldots p_{i}^{\alpha_{i}}} \\
& -\sum_{\alpha_{1}, \alpha_{2}, \ldots \alpha_{i}}(-1)^{\alpha_{1}+\alpha_{2}+\cdots+\alpha_{i}} 2^{n: p_{i}+1} p_{1}^{\alpha_{1}} p_{2}^{\alpha_{2}} \ldots p_{i}^{\alpha_{i}} \\
& a_{i+1}=0 \\
& =\sum_{\alpha_{1}, \alpha_{2}, \ldots \alpha_{i}}(-1)^{\alpha_{1}+\alpha_{2}+\cdots+\alpha_{i}+\alpha_{i+1}} 2^{n: p_{1}^{\alpha_{1}} p_{2}^{\alpha_{2}} \cdots p_{i}^{\alpha_{i}} p_{i+1}^{\alpha_{i+1}}} \\
& \quad+\sum_{i+1}(-1 \\
& \sum_{\alpha_{1}, \alpha_{2}, \ldots \alpha_{i}}(-1)^{\alpha_{1}+\alpha_{2}+\cdots+\alpha_{i}+\alpha_{i+1}} 2^{n: p_{1}^{\alpha_{1}} p_{2}^{\alpha_{2}} \cdots p_{i}^{\alpha_{i}} p_{i+1}^{\alpha_{i+1}}} \\
& =\sum_{\alpha_{1}, \alpha_{2}, \ldots \alpha_{i}, \alpha_{i+1}}(-1)^{\alpha_{1}+\alpha_{2}+\cdots+\alpha_{i}+\alpha_{i+1}} 2^{n: p_{1}^{\alpha_{1}} p_{2}^{\alpha_{2}} \cdots p_{i}^{\alpha_{i}} p_{i+1}^{\alpha_{i+1}},}
\end{aligned}
$$

wo $\alpha_{1}, \alpha_{2}, \ldots \alpha_{i}, \alpha_{i+1}$ unabhängig voneinander die Werte 0 und 1 annehmen. Die Formel 3) bleibt also auch für $i+1$ richtig, wenn sie für $i$ gilt; nun gilt sie nach 2) für $i=2$, mithin allgemein für $i \geqq 2$. Für $i=1$ aber gilt die Formel 1), die, wenn $\frac{n}{p_{1}}$ zugleich mit $n$ gerade oder ungerade ist, mit 3 ) (wenn darin für $i 1$ genommen wird) gleichbedeutend ist und nur, wenn $n$ gerade und $\frac{n}{p_{1}}$ ungerade ist, gleich $2^{n}-2^{n: p_{1}}-2$ ist.

Nunmehr bleibt nach der $k$-ten Ausscheidung ein $(k+1)$-ter Komplex übrig, der nur noch diejenigen $n$-ten Koinzidenz-Repräsentanten enthält, welche weder $\left(\frac{n}{p_{1}}\right)$-te, noch $\left(\frac{n}{p_{2}}\right)$-te, noch $\left(\frac{n}{p_{3}}\right)$-te, 
... noch $\left(\frac{n}{p_{k}}\right)$-te und welche also sämtlich primitive $n$-te sind und deren Anzahl beträgt:

$$
\sum_{\alpha_{1}, \alpha_{2}, \ldots \alpha_{k}}(-1)^{\alpha_{1}+\alpha_{2}+\cdots+\alpha_{k}} 2^{n: p_{1}^{\alpha_{1}}} p_{2}^{\alpha_{2}} \ldots p_{k}^{\alpha_{k}}
$$

(wo $\alpha_{1}, \alpha_{2}, \ldots \alpha_{k}$ unabhängig voneinander die Werte 0 und 1 annehmen sollen), wenn $k \geqq 2$, also $n$ mindestens aus zwei voneinander verschiedenen Primzahlen zusammengesetzt ist, oder wenn zwar $k=1$, aber $\frac{n}{p_{1}}$ zugleich mit $n$ gerade oder ungerade ist. Die Formel 4) gilt nur dann nicht, wenn $k=1, n$ gerade und $\frac{n}{p_{1}}$ ungerade ist, also $n=p_{1}=2$; in diesem Falle gilt die Formel 1), welche dann in $2^{2}-2-2=0$ übergeht.

Mithin haben wir:

Die Anzahl der primitiven $n$-ten Koinzidenz-Repräsentanten ist gleich

$$
\sum_{\alpha_{1}, \alpha_{2}, \ldots \alpha_{k}}(-1)^{\alpha_{1}+\alpha_{2}+\cdots+\alpha_{k}} 2^{n: p_{1}^{\alpha_{1}} p_{2}^{\alpha_{2}} \ldots p_{k}^{\alpha_{k}}}{ }_{\left(\alpha_{1}, \alpha_{2}, \ldots \alpha_{k}=0,1\right),}
$$

wenn $n=p_{1}^{\pi_{1}} p_{2}^{\pi_{2}} p_{3}^{\pi_{3}} \ldots p_{k}^{\pi_{k}}>2$ ist, und gleich 0 , wenn $n=2$ ist.

Anmerkung. Die Summe in 4) wird aus der bekannten zahlenTheoretischen Funktion $\varphi(n)=n\left(1-\frac{1}{p_{1}}\right)\left(1-\frac{1}{p_{2}}\right) \cdots\left(1-\frac{1}{p_{k}}\right)$ Tolgender Weise gebildet: die absoluten Beträge der einzelnen Glieder des entwickelten Produktes $\varphi(n)$ werden für die Summe in 4) als Exponenten zu 2, als Grundzahl, genommen, und die so entstehenden Potenzen von 2 werden mit demselben Vorzeichen [in der Summe in 4)] versehen wie ihre Exponenten im entwickelten Produkte $\varphi(n)$.

Die Anzahl der primitiven $n$-ten Koinzidenz-Repräsentanten läßt sich auch auf folgende Weise bestimmen.

Hängen zwei zahlentheoretische Funktionen $F(n)$ und $f(n)$ durch die Relation

$$
F(n)=\sum f(d)
$$

zusammen, wo das Summenzeichen sich auf alle Divisoren $d$ (inkl. $n$ ) der Zahl $n$ bezieht, und ist $n=p_{1}^{\pi_{1}} p_{2}^{\pi_{2}} \cdots p_{k}^{\pi_{k}}$, so ist bekanntlich: 


$$
\begin{gathered}
f(n)=F(n)-\left[F\left(\frac{n}{p_{1}}\right)+\cdots+F\left(\frac{n}{p_{k}}\right)\right]+\left[F\left(\frac{n}{p_{1} p_{2}}\right)+\cdots\right. \\
\left.+F\left(\frac{n}{p_{k-1} p_{k}}\right)\right]-\left[F\left(\frac{n}{p_{1} p_{2} p_{3}}\right)+\cdots+F\left(\frac{n}{p_{k-2} p_{k-1} p_{k}}\right)\right]+\cdots,
\end{gathered}
$$

was wir auch kürzer so schreiben können:

$$
f(n)=\sum_{\alpha_{1}, \alpha_{2}, \ldots \alpha_{k}}(-1)^{\alpha_{1}+\alpha_{2}+\cdots+\alpha_{k}} F\left(\frac{n}{p_{1}^{\alpha_{1}} p_{2}^{\alpha_{2}} \cdots p_{k}^{\alpha_{k}}}\right),
$$

wo $\alpha_{1}, \alpha_{2}, \ldots \alpha_{k}$ unabhängig voneinander die Werte 0 und 1 annehmen sollen.

Nun bestehen (nach Nr. 30 und Anfang dieser Nummer) die $n$-ten Koinzidenz-Repräsentanten, deren Anzahl $2^{n}-(-1)^{n}$ beträgt (Satz 19 in Nr.29), aus den sämtlichen primitiven $d$-ten Koinzidenz-Repräsentanten und nur aus diesen, wo $d$ irgendein Divisor von $n$ ( $n$ selbst nicht ausgeschlossen) bedeutet. Wenn daher die Anzahl $2^{n}-(-1)^{n}$ der $n$-ten Koinzidenz-Repräsentanten mit $F(n)$ bezeichnet wird und die Anzahl der primitiven $n$-ten Koinzidenz-Repräsentanten mit $f(n)$, so muß sein:

$$
\sum f(d)=F(n)=2^{n}-(-1)^{n},
$$

wo das Summenzeichen sich auf alle Divisoren der Zahl $n$ bezieht, und folglich muß die Anzahl der primitiven $n$-ten KoinzidenzRepräsentanten sein:

$$
\alpha)\left\{\begin{array}{c}
f(n)=\sum_{\alpha_{1}, \alpha_{2}, \ldots \alpha_{k}}(-1)^{\alpha_{1}+\alpha_{2}}+\cdots+\alpha_{k} F\left(\frac{n}{p_{1}^{\alpha_{1}} p_{2}^{\alpha_{\vartheta}} \cdots p_{k}^{\alpha_{k}}}\right) \\
=\sum_{\alpha_{1}, \alpha_{2}, \ldots \alpha_{k}}(-1)^{\alpha_{1}+\alpha_{2}+\cdots+\alpha_{k}}\left[2^{n: p_{1}^{\alpha_{1}} p_{2}^{\alpha_{2}} \ldots p_{k}^{\alpha_{k}}}\right. \\
-(-1)^{\left.n: p_{1}^{\alpha_{1}} p_{2}^{\alpha_{2}} \ldots p_{k}^{\alpha_{k}}\right]}
\end{array}\right.
$$

wo $\alpha_{1}, \alpha_{2}, \ldots \alpha_{k}$ unabhängig voneinander die Werte 0 und 1 annehmen sollen.

Wählen wir nun die Bezeichnung der $k$ in $n$ aufgehenden, voneinander verschiedenen Primzahlen so, daß $1<p_{1}<p_{2}<\ldots<p_{k}$ ist, so sind stets $n$ und $\frac{n}{p_{2}^{\alpha_{2}} \ldots p_{k}^{\alpha_{k}}}$ beide zugleich gerade oder ungerade und ebenso $\frac{n}{p_{1}}$ und $\frac{n}{p_{1} p_{2}^{\alpha_{2}} \ldots p_{k}^{\alpha}}$; mithin ist

$$
\sum_{\alpha_{1}, \alpha_{2}, \ldots \alpha_{k}}(-1)^{\alpha_{1}+\alpha_{2}+\cdots+\alpha_{k}}(-1)^{n: p_{1}^{\alpha_{1}} p_{2}^{\alpha_{2}} \cdots p_{k}^{\alpha_{k}}}
$$




$$
\begin{aligned}
& =\sum_{\alpha_{2}, \ldots \alpha_{k}}^{\alpha_{1}=0}(-1)^{\alpha_{2}+\cdots+\alpha_{k}}(-1)^{n: p_{2}^{\alpha_{2}} \ldots p_{k}^{\alpha_{k}}}
\end{aligned}
$$

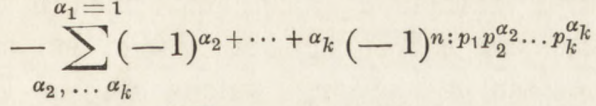

$$
\begin{aligned}
& =(-1)^{n} \sum_{\alpha_{2}, \ldots \alpha_{k}}(-1)^{\alpha_{2}+\cdots+\alpha_{k}}-(-1)^{n: p_{1}} \sum_{\alpha_{2}, \ldots \alpha_{k}}(-1)^{\alpha_{2}+\cdots+\alpha_{k}} \\
& =\left[(-1)^{n}-(-1)^{n: p_{1}}\right] \sum_{\alpha_{2}, \ldots \alpha_{k}}(-1)^{\alpha_{2}+\cdots+\alpha_{k}} \\
& =\left[(-1)^{n}-(-1)^{n: p_{1}}\right] \cdot\left[1-(k-1)+\left(\begin{array}{c}
k-2 \\
2
\end{array}\right)-+\cdots\right. \\
& \left. \pm\left(\begin{array}{l}
k-1 \\
k-1
\end{array}\right)\right]=\left[(-1)^{n}-(-1)^{n: p}\right](1-1)^{k-1} \text {. }
\end{aligned}
$$

Dieses letzte Produkt ist aber $=0$, wenn $k>1$ oder zwar $k=1$, aber $n$ und $\frac{n}{p_{1}}$ beide zugleich gerade oder ungerade sind, und nur dann ist dieses Produkt von Null verschieden, wenn $k=1$, $n$ gerade und $n: p_{1}$ ungerade, wenn also $n=p_{1}=2$ ist, und zwar ist dann dieses Produkt $=2$.

Mithin geht $\alpha$ ) über in

$$
f(n)=\sum_{\alpha_{1}, \alpha_{2}, \ldots \alpha_{k}}(-1)^{\alpha_{1}+\alpha_{2}+\cdots+\alpha_{k}} 2^{n: p_{1}^{\alpha_{1}} p_{2}^{\alpha_{2}} \ldots p_{k}^{\alpha_{k}},}
$$

wenn $n>2$ ist, und für $n=2$ in

$$
f(2)=\sum_{\alpha}(-1)^{\alpha} 2^{2^{1-\alpha}}-2=2^{2}-2-2=0
$$

welche Resultate mit den vorher gefundenen übereinstimmen.

32. Weil die primitiven $n$-ten Koinzidenz-Repräsentanten in Gruppen von je $n$ zerfallen, so daß jeder primitive $n$-te in einer und nur in einer dieser Gruppen enthalten ist (Nr.30), so muß die Summe in 4), die die Anzahl der primitiven $n$-ten angibt, durch $n$ teilbar sein. Dividieren wir nun die Summe in 4) durch $(-1)^{k} 2^{n: p_{1} p_{2} \ldots p_{k}}$, so erhellt:

Ist $n=p_{1}^{\pi_{1}} p_{2}^{\pi_{2}} \cdots p_{k}^{\pi_{k}}$, wo $p_{1}<p_{2}<\cdots<p_{k}$, so ist, je nachdem $n$ ungerade oder gerade und also $p_{1}=2$ ist:

$\sum_{\alpha_{1}, \alpha_{2}, \ldots \alpha_{k}}(-1)^{\alpha_{1}+\alpha_{2}+\cdots+\alpha_{k}} 2^{n\left(p_{1}^{\alpha_{1}} p_{2}^{\alpha_{2}} \ldots p_{k}^{\alpha} \alpha_{k}-1\right): p_{1} p_{2} \ldots p_{k}}$
$\equiv 0(\bmod . n$ oder $\equiv 0\left(\bmod . n\right.$ oder mod. $\left.n: 2^{\pi_{1}}\right)$, wo $\alpha_{1}, \alpha_{2}, \ldots \alpha_{k}$ unabhängig voneinander die Werte 0 und 1 annehmen sollen.

Berliner, Habilitationsschrift. 


\section{$\S 6$.}

33. Bilden die drei Strahlen $g_{1}, g_{2}, g_{3}$ ein Tripel in $(P)^{3}$, wo $P$ ein auf keiner Seite von $A B C$ liegender Punkt ist, so bilden ihre ersten Repräsentanten $g_{1}^{(1)}, g_{2}^{(1)}, g_{3}^{(1)}$, welche die ein Tripel in der induzierten Involution $\left(p^{2}\right)^{3}$ bildenden Pole $G_{1}, G_{2}, G_{3}$ von $g_{1}, g_{2}, g_{3}$ aus $P$ projizieren, gleichfalls ein Tripel in $(P)^{3}$ (Satz 10 in Nr. 17); und zwar muß der Sinn $g_{1}^{(1)} g_{2}^{(1)} g_{3}^{(1)}$ mit dem Sinne $g_{1} g_{2} g_{3}$ übereinstimmen. Denn der zu $g_{3}$ in $(P)^{2}$ zugepaarte Strahl $g_{3}^{\prime}$ ist von $g_{3}$ durch $g_{1}$ und $g_{2}$ harmonisch getrennt (Nr.4) und ebenso der zu $g_{1}$ in $(P)^{2}$ zugepaarte $g_{1}^{\prime}$ von $g_{1}$ durch $g_{2}$ und $g_{3}$; mithin liegt $g_{3}^{\prime}$ in demjenigen von $g_{1}$ und $g_{2}$ gebildeten vollkommenen Winkel, innerhalb dessen $g_{3}$ und $g_{1}^{\prime}$ nicht liegen, und die Sinne $g_{1} g_{2} g_{3}$ und $g_{1} g_{3}^{\prime} g_{2}$ stimmen miteinander überein. Wenn nun ein Strahl $g$ von $P$ denjenigen von $g_{1}$ und $g_{2}$ gebildeten Winkel durchläuft, innerhalb dessen $g_{3}^{\prime}$ liegt, so beschreibt sein erster Repräsentant $g^{(1)}$ im entgegengesetzten Sinne (Nr.26) um $P$ denjenigen von $g_{1}^{(1)}$ und $g_{2}^{(1)}$ gebildeten vollkommenen Winkel, innerhalb dessen, weil $g_{3}$ und $g_{3}^{\prime}$ einen und denselben ersten Repräsentanten haben, $g_{3}^{(1)}$ liegt, und nur diesen Winkel; da $g$ bei seiner Bewegung den zu $g_{1}$ in $(P)^{2}$ zugepaarten $g_{1}^{\prime}$ nicht erreicht und mithin $g^{(1)}$ keinen vollen Umlauf um $P$ vollenden kann (Nr.26) ${ }^{1}$ ). Es muß demnach der Sinn $g_{j}^{(1)} g_{3}^{(1)} g_{2}^{(1)}$ zum Sinne $g_{1} g_{3}^{\prime} g_{2}$ und also auch zu $g_{1} g_{2} g_{3}$ entgegengesetzt sein, mithin stimmen die Sinne $g_{1}^{(1)} g_{2}^{(1)} g_{3}^{(1)}$ und $g_{1} g_{2} g_{3}$ miteinander überein. (Diese Resultate ergeben sich auch aus Satz 15 in Nr.26, wenn man bemerkt, daß die drei Ordinatenwinkel eines Strahlentripels von $(P)^{3}$ sich voneinander um $\frac{\pi}{3}$ unterscheiden.)

Nunmehr müssen auch die zweiten Repräsentanten $g_{1}^{(2)}, g_{2}^{(2)}$, $g_{3}^{(2)}$, die die ersten Repräsentanten von $g_{1}^{(1)}, g_{2}^{(1)}, g_{3}^{(1)}$ sind, und mithin auch die dritten, vierten und überhaupt die $n$-ten Repräsentanten $g_{1}^{(n)}, g_{2}^{(n)}, g_{3}^{(n)}$ je ein mit $g_{1} g_{2} g_{3}$ gleichen Sinn habendes Tripel in $(P)^{3}$ bilden.

1) Das hier benutzte Verfahren kann auch zu einem zweiten Beweise (s. oben Nr. 26) dafür dienen, daß die Bewegungen eines Strahles und dessen ersten Repräsentanten im Büschel einander entgegengesetzt sind, wenn man von dem Tripel der Ecktransversalen $p_{A} p_{B} p_{C}$ von $(P)^{3}$ ausgeht, welche Ecktransversalen mit ihren ersten Repräsentanten zusammenfallez. 
Dies gilt nun ohne weiteres auch von den drei $n$-ten Repräsentanten eines Punkttripels in der von $A B C$ auf einer durch keine Ecke von $A B C$ gehenden Geraden erzeugten Involution dritten Grades.

34. Bilden nun in einem Gebilde erster Stufe die Elemente $Q_{1}, Q_{2}, Q_{3}$ ein Tripel in der von $A B C$ im Gebilde erzeugten Involution dritten Grades und ist $Q_{1}^{(i)} \equiv Q_{1}$ bzw. $Q_{1}^{(i)} \equiv Q_{2}$ bzw. $Q_{1}^{(i)} \equiv Q_{3}$, so müssen $Q_{2}^{(i)} \equiv Q_{2}$ und $Q_{3}^{(i)} \equiv Q_{3}$ bzw. $Q_{2}^{(i)} \equiv Q_{3}$ und $Q_{3}^{(i)} \equiv Q_{1}$ bzw. $Q_{2}^{(i)} \equiv Q_{1}$ und $Q_{3}^{(i)} \equiv Q_{2}$ sein. Denn $Q_{1}^{(i)} Q_{2}^{(i)} Q_{3}^{(i)}$ bilden, wie wir sahen, ein mit $Q_{1} Q_{2} Q_{3}$ gleichen Sinn habendes Tripel in der Involution dritten Grades; diese beiden Tripel müssen nun zusammenfallen, wenn sie ein Element gemein haben, und alsdann müssen $Q_{1}^{(i)}, Q_{2}^{(i)}, Q_{3}^{(i)}$ der Reihe nach entweder mit $Q_{1}, Q_{2}, Q_{3}$ identisch sein, oder aus diesen durch eine zyklische Vertauschung hervorgehen. Mithin:

Satz 20. Bilden $Q_{1} Q_{2} Q_{3}$ ein Tripel in der von $A B C$ im Gebilde erzeugten Involution dritten Grades und ist $Q_{1}$ ein primitiver $n$-ter Koinzidenz-Repräsentant, so sind auch $Q_{2}$ und $Q_{3}$ primitive $n$-te Koinzidenz-Repräsentanten.

Ist ferner $Q_{1}^{(k)}$ der niedrigste Repräsentant von $Q_{1}$, welcher mit einem der beiden Elemente $Q_{2}$ und $Q_{3}$ zusammenfällt (wo also ein noch niedriger als der $k$-te Repräsentant von $Q_{1}$ weder mit $Q_{2}$ noch mit $Q_{3}$ zusammenfällt), so ist $Q_{1}^{(2 k)}$ der niedrigste Repräsentant von $Q_{1}$, welcher mit $Q_{3}$ bzw. $Q_{2}$, und $Q_{1}^{(3 k)}$ der niedrigste, welcher (in beiden Fällen) mit $Q_{1}$ zusammenfällt. Das Nämliche gilt dann von $Q_{2}$ und von $Q_{3}$, nur sind im selben überall $Q_{1}$, $Q_{2}, Q_{3}$ für $Q_{2}$ durch $Q_{2}, Q_{3}, Q_{1}$ und für $Q_{3}$ durch $Q_{3}, Q_{1}$, $Q_{2}$ zu ersetzen; es sind also dann $Q_{1}, Q_{2}, Q_{3}$ primitive $(3 k)$-te Koinzidenz-Repräsentanten.

In der Tat, wäre etwa $Q_{2}$ schon ein $i$-ter Koinzidenz-Repräsentant $(i<n)$, so müßte es auch $Q_{1}$ sein; $Q_{2}$ und $Q_{3}$ müssen also zugleich mit $Q_{1}$ primitive $n$-te Koinzidenz-Repräsentanten sein. Wenn ferner etwa $Q_{1}^{(k)} \equiv Q_{2}$ ist, muß $Q_{1}^{(2 k)} \equiv Q_{1}^{(k)(k)} \equiv Q_{2}^{(k)} \equiv Q_{3}$ und $Q_{1}^{(3 k)} \equiv Q_{1}^{(2 k)(k)} \equiv Q_{3}^{(k)} \equiv Q_{1}$ sein; wäre schon $Q_{1}^{(l)} \equiv Q_{3}$, wo $2 k>l>k$ wegen der Voraussetzung des Satzes, so müßte, weil $Q_{1}^{(l)} \equiv Q_{3} \equiv Q_{1}^{(2 k)}$, $Q_{3}$ und mithin auch $Q_{1}$ ein $(2 k-l)$-ter Koinzidenz-Repräsentant 
sein, und $Q_{2} \equiv Q_{1}^{(k)}$ müßte gegen die Voraussetzung schon unter den $2 k-l-1$ Repräsentanten $Q_{1}^{(1)}, Q_{1}^{(2)}, Q_{1}^{(3)}, \ldots Q_{1}^{(2 k-l-1)}$ von $Q_{1}$ vorkommen (Nr. 30), wo $2 k-l<k$ ist; es muß also $Q_{1}^{(2 k)}$ der niedrigste Repräsentant von $Q_{1}$ sein, für den $Q_{1}^{(2 k)} \equiv Q_{3}$ ist. Wäre dann $Q_{1}$ ein primitiver $n$-ter Koinzidenz-Repräsentant, wo $n<3 k$, so müßte einerseits, weil nach dem soeben Bewiesenen $Q_{3} \equiv Q_{1}^{(2 k)}$ nicht unter den Repräsentanten von $Q_{1}$, die niedriger als der $(2 k)$-te sind, vorkommen kann, $n>2 k$ und andererseits, weil $Q_{2}^{(3 k)} \equiv Q_{1}$ ist, $n$ ein Teiler von $3 k$ sein, was unmöglich ist; $Q_{1}$ und mithin auch $Q_{2}$ und $Q_{3}$ müssen also primitive $(3 k)$-te Koinzidenz-Repräsentanten sein. Wenn endlich dann $Q_{2}^{(i)} \equiv Q_{3}$ oder $\equiv Q_{1}$ wäre, wo $i<k$, so müßte nach dem eben Bewiesenen $Q_{2}$ ein primitiver (3i)-ter Koinzidenz-Repräsentant sein; $Q_{2}^{(k)} \mathrm{muß}$ also dann der niedrigste Repräsentant von $Q_{2}$ sein, für den $Q_{2}^{(k)} \equiv Q_{1}^{(2 k)} \equiv Q_{3}$ ist. Aus dem vorstehenden Satze folgt nun ohne weiteres:

Satz 21. Ist $Q_{1}$ ein primitiver $n$-ter KoinzidenzRepräsentant, so kommt weder $Q_{2}$, noch $Q_{3}$ unter den Repräsentanten von $Q_{1}$ vor, wenn $n$ kein Vielfaches von 3 ist; ist aber $n$ ein Vielfaches von 3, so kommt entweder keiner der beiden Elemente $Q_{2}$ und $Q_{3}$, oder jeder derselben unter den Repräsentanten von $Q_{1}$ vor, und zwar muß im letztern Falle, wo $Q_{2}$ und $Q_{3}$ vorkommen, entweder $Q_{1}^{(n: 3)} \equiv Q_{2}$ und $Q_{1}^{(2 n: 3)} \equiv Q_{3}$, oder $Q_{1}^{(n: 3)} \equiv Q_{3}$ und $Q_{1}^{(2 n: 3)} \equiv Q_{2}$ sein.

35. Ist $n$ ein Vielfaches von 3 und ist $Q_{1}$ ein solcher primitiver $n$-ter Koinzidenz-Repräsentant, für den $Q_{1}^{(n: 3)} \equiv Q_{2}$ oder $\equiv Q_{3}$ ist und mithin $Q_{1}^{(2 n: 3)} \equiv Q_{3}$ bzw. $\equiv Q_{2}$, so bilden auch $Q_{1}^{(k)}, Q_{1}^{(k+n: 3)}$, $Q_{1}^{(k+2 n: 3)}$ für jeden Wert von $k$ ein ebensolches Tripel (sogar dem Sinne nach) wie $Q_{1} Q_{2} Q_{3}$ bzw. wie $Q_{1} Q_{3} Q_{2}$. Denn es ist $Q_{1}^{(k+n: 3)} \equiv Q_{1}^{(n: 3)(k)} \equiv Q_{2}^{(k)}$ bzw. $\equiv Q_{3}^{(k)}$ und $Q_{1}^{(k+2 n: 3)} \equiv Q_{1}^{(2 n: 3)(k)} \equiv Q_{3}^{(k)}$ bzw. $\equiv Q_{2}^{(k)}$ und die $k$-ten Repräsentanten $Q_{1}^{(k)} Q_{2}^{(k)} Q_{3}^{(k)}$ des Tripels $Q_{1} Q_{2} Q_{3}$ bilden ein mit dem letzten gleichen Sinn habendes Tripel. Mithin muß jede der oben (Nr.30) definierten Gruppen von je $n$ primitiven $n$-ten Koinzidenz-Repräsentanten entweder kein einziges solches Element $Q_{1}$ enthalten, unter dessen Repräsentanten $Q_{2}$ und mithin auch $Q_{3}$ vorkommen, oder aus lauter solchen Elementen bestehen. Mithin:

Diejenigen primitiven $n$-ten Koinzidenz-Repräsentanten $Q_{1}$, unter deren Repräsentanten $Q_{2}$ und $Q_{3}$ vorkommen, wo $n$ 
notwendig ein Vielfaches von 3 ist, können nur in einer solchen Anzahl auftreten, welche ein ganzes Vielfaches von $n$ ist.

36. Wir wollen nun, wenn $n$ ein Vielfaches von 3 ist, die Anzahl derjenigen primitiven $n$-ten Koinzidenz-Repräsentanten $Q_{1}$ bestimmen, unter deren Repräsentanten $Q_{2}$ und mithin auch $Q_{3}$ vorkommen. Diese Anzahl stimmt nach den Sätzen 20 und 21 mit der Anzahl derjenigen Elemente $Q_{1}$ überein, für welche je $\operatorname{der}\left(\frac{n}{3}\right)$-te (und kein niedriger) Repräsentant $Q_{1}^{(n: 3)}$ mit $Q_{2}$ oder $Q_{3}$ zusammenfällt (wo also ein niedriger als der ( $n: 3)$-te Repräsentant von $Q_{1}$ weder mit $Q_{2}$ noch mit $Q_{3}$ zusammenfällt). Die letztere Anzahl kann nun wie folgt bestimmt werden.

Sind $\omega_{1}, \omega_{2}$ und $\omega_{3}$ die Ordinatenwinkel von $Q_{1}, Q_{2}$ und $Q_{3}$, wo die bei der Bestimmung der Ordinatenwinkel hinzugedachten Bewegungen aller drei Elemente in einem und demselben Sinne folgen sollen und dieser Sinn und die Bezeichnung der Indices der Elemente so getroffen sein sollen, daß $0<\omega_{1}<\omega_{2}<\omega_{3}$ ist, wo also, weil $Q_{1} Q_{2} Q_{3}$ von dem oben (Nr. 26) definierten Punkte $R$, dem Scheitel aller Ordinatenwinkel, aus durch einen regelmäßigen Dreistrahl projiziert werden (Nr.4):

$$
\omega_{2}=\omega_{1}+\frac{\pi}{3}, \quad \omega_{3}=\omega_{1}+\frac{2 \pi}{3},
$$

und soll der $k$-te Repräsentant von $Q_{1}$ mit $Q_{2}$ oder $Q_{3}$ zusammenfallen, soll also $Q_{1}^{(k)} \equiv Q_{2}$ oder $\equiv Q_{3}$ sein, so wird dann (nach Satz 15 in Nr. 26), wenn $k$ ungerade ist:

bzw.

$$
\begin{gathered}
-2^{k} \omega_{1}=\omega_{1}^{(k)}=-\left(\pi-\omega_{2}+i \pi\right) \\
=-\left(\pi-\omega_{1}-\frac{\pi}{3}+i \pi\right)=-\left(\frac{3 i+2}{3} \pi-\omega_{1}\right)
\end{gathered}
$$

$$
\begin{gathered}
-2^{k} \omega_{1}=\omega_{1}^{(k)}=-\left(\pi-\omega_{3}+i \pi\right) \\
=-\left(\pi-\omega_{1}-\frac{2 \pi}{3}+i \pi\right)=-\left(\frac{3 i+1}{3} \pi-\omega_{1}\right)
\end{gathered}
$$

sein müssen, wo $i$ die Anzahl der vollen Umläufe von $Q_{1}^{(k)}$ im Gebilde angibt und wo $i$ auch 0 sein kann, also:

$$
\omega_{1}=\frac{3 i+2}{3\left(2^{k}+1\right)} \pi \quad \text { bzw. } \quad \omega_{1}=\frac{3 i+1}{3\left(2^{k}+1\right)} \pi,
$$


wobei, weil nur $\omega_{1}<\pi$ in Betracht kommt, $3 i+2<3.2^{k}+3$ bzw. $3 i+1<3.2^{k}+3$, also $i<2^{k}+\frac{1}{3}$ bzw. $i<2^{k}+\frac{2}{3}$, mithin (in beiden Fällen), weil $i$ nur eine ganze Zahl sein kann, $i \leqq 2^{k}$, also $i=0,1,2, \ldots 2^{k}$, und, wenn $k$ gerade ist:

$$
2^{k} \omega_{1}=\omega_{1}^{(k)}=\omega_{2}+i \pi=\omega_{1}+\frac{\pi}{3}+i \pi=\frac{3 i+1}{3} \pi+\omega_{1}
$$

bzw.

$$
2^{k} \omega_{1}=\omega_{1}^{(k)}=\omega_{3}+i \pi=\omega_{1}+\frac{2 \pi}{3}+i \pi=\frac{3 i+2}{3} \pi+\omega_{1},
$$

also:

$$
\omega_{1}=\frac{3 i+1}{3\left(2^{k}-1\right)} \pi \quad \text { bzw. } \quad \omega_{1}=\frac{3 i+2}{3\left(2^{k}-1\right)} \pi,
$$

wobei $3 i+1<3.2^{k}-3$ bzw. $3 i+2<3.2^{k}-3$, also $i<2^{k}-\frac{4}{3}$ bzw. $i<2^{k}-\frac{5}{3}$, mithin (in beiden Fällen) $i \leqq 2^{k}-2$, also $i=0,1,2, \ldots 2^{k}-2$. Hat, umgekehrt, der Ordinatenwinkel $\omega_{1}$ eines Elementes $Q_{1}$ die Form $\frac{(3 i+r) \pi}{3\left[2^{k}-(-1)^{k}\right]}$, wo $0<r<3$, so ist dann:

$$
\begin{gathered}
\omega_{1}^{(k)}=(-2)^{k} \omega_{1}=\frac{(-2)^{k}(3 i+r) \pi}{3\left[2^{k}-(-1)^{k}\right]} \\
=\frac{(-1)^{k}\left[2^{k}-(-1)^{k}\right](3 i+r) \pi+(3 i+r) \pi}{3\left[2^{k}-(-1)^{k}\right]}
\end{gathered}
$$

$=(-1)^{k}\left(i+\frac{r}{3}\right) \pi+\frac{(3 i+r) \pi}{3\left[2^{k}-(-1)^{k}\right]}=(-1)^{k}\left[(-1)^{k} \omega_{1}+\frac{r \pi}{3}+i \pi\right]$ und dies ist $=\omega_{1}+\frac{r \pi}{3}+i \pi$, wenn $k$ gerade ist (also $=\omega_{2}+i \pi$ oder $=\omega_{3}+i \pi$, je nachdem $r=1$ oder $r=2$ ist), und $=-\left(\pi-\omega_{1}-\frac{3-r}{3} \pi+i \pi\right)$, wenn $k$ ungerade ist (also $=-\left(\pi-\omega_{2}+i \pi\right)$ oder $=-\left(\pi-\omega_{3}+i \pi\right)$, je nachdem $r=2$ oder $r=1$ ist); mithin muß dann in allen Fällen $Q_{1}^{(k)} \equiv Q_{2}$ oder $\equiv Q_{3}$ sein.

Es gibt also, je nachdem $k$ ungerade oder gerade ist, $2\left(2^{k}+1\right)$ oder $2\left(2^{k}-1\right)$ Elemente $Q_{1}$, für welche je $Q_{1}^{(k)}$ mit einem der beiden Elemente $Q_{2}$ und $Q_{3}$ zusammenfällt. 
Soll nun für ein Element $Q_{1}$, für das $Q_{1}^{(k)} \equiv Q_{2}$ oder $\equiv Q_{3}$ ist, $Q_{1}^{(i)}$ der niedrigste mit einem der beiden Elemente $Q_{2}$ und $Q_{3}$ zusammenfallende Repräsentant von $Q_{1}$ sein, wo notwendig $i<k$ ist, so wird dann $k$ ein Vielfaches von $i$, nicht aber von $3 i$ sein; und alsdann wird auch für jede Zahl $m$, die ein Vielfaches von $i$, nicht aber von $3 i$ ist, $Q_{1}^{(m)}$ mit einem der beiden Elemente $Q_{2}$ und $Q_{3}$ zusammenfallen. Denn alsdann muß $Q_{1}$ ein primitiver (3i) - ter Koinzidenz-Repräsentant sein (Satz 20 in Nr. 34) und also muß, wenn $k=q 3 i+r(r<3 i), Q_{2}$ oder $Q_{3} \equiv Q_{1}^{(k)} \equiv Q_{1}^{(q 3 i+r)}$ $\equiv Q_{1}^{(q 3 i)(r)} \equiv Q_{1}^{(r)}$ sein; mithin muß entweder $r=i$, oder $r=2 i$ sein (ebenda), also $k=(q 3+1) i$ oder $k=(q 3+2) i$; und ist, umgekehrt, etwa $m=q 3 i+2 i$, so $\mathrm{mu}, Q_{1}^{(m)} \equiv Q_{1}^{(q 3 i+2 i)} \equiv Q_{1}^{(q 3 i)(2 i)}$ $\equiv Q_{1}^{(2 i)} \equiv Q_{2}$ oder $\equiv Q_{3}$ sein, je nachdem $Q_{1}^{(i)} \equiv Q_{3}$ oder $\equiv Q_{2}$ ist. Mithin wird dann auch mindestens einer der folgenden Repräsentanten von $Q_{1}: Q_{1}^{\left(k: p_{1}\right)}, Q_{1}^{\left(k: p_{2}\right)}, Q_{1}^{\left(k: p_{3}\right)}, \ldots Q_{1}^{\left(k: p_{s}\right)}$, niemals aber $Q_{1}^{(k: 3)}$ mit einem der beiden Elemente $Q_{2}$ und $Q_{3}$ zusammenfallen, wenn

$$
k=3^{\pi} p_{1}^{\pi_{1}} p_{2}^{\pi_{2}} p_{3}^{\pi_{3}} \cdots p_{s}^{\pi_{s}}
$$

ist, wo $\pi \geqq 0$, hingegen $\pi_{1} \geqq 1, \pi_{2} \geqq 1, \ldots \pi_{s} \geqq 1$; denn $k$ darf kein Vielfaches von $3 i$ und also $\frac{k}{3}$ kein Vielfaches von $i$ sein, $k$ ist aber ein Vielfaches von $i$ und $>i$, mithin mu mindestens eine der Zahlen $\frac{k}{p_{1}}, \frac{k}{p_{2}}, \cdots \frac{k}{p_{s}}$, welche ebenso wie $k$ keine Vielfache von $3 i$ sind, ein Vielfaches von $i$ sein. Ist, umgekehrt, einer oder mehrere der Repräsentanten $Q_{1}^{\left(k: p_{1}\right)}, Q_{1}^{\left(k: p_{2}\right)}, \ldots Q_{1}^{\left(k: p_{s}\right)}$ mit $Q_{2}$ oder $Q_{3}$ identisch, so muß auch $Q_{1}^{(k)}$ mit einem der beiden Elemente $Q_{2}$ und $Q_{3}$ identisch sein; denn, wenn $\frac{k}{p_{1}}$, oder $\frac{k}{p_{2}}, \ldots$ oder $\frac{k}{p_{s}}$ ein Vielfaches von $i$, nicht aber von $3 i$ ist, gilt dasselbe auch von $k$. Wenn aber für ein Element $Q_{1}$, für das $\left(_{1}^{(k)} \equiv Q_{2}\right.$ oder $\equiv Q_{3}$ ist, weder $Q_{1}^{\left(k: p_{1}\right)}$, noch $Q_{1}^{\left(k: p_{2}\right)}, \ldots$ noch $Q_{1}^{\left(k: p_{s}\right)}$ mit einem der beiden Elemente $Q_{2}$ und $Q_{3}$ zusammenfällt, so muß, nach dem eben Bewiesenen, $Q_{1}^{(k)}$ der niedrigste mit einem der beiden Elemente $Q_{2}$ und $Q_{3}$ zusammenfallende Repräsentant von $Q_{1}$ sein.

Um nun diejenigen Elemente $Q_{1}$ zu erhalten, für welche $Q_{1}^{(k)}$ der niedrigste mit einem der beiden Elemente $Q_{2}$ und $Q_{3}$ zusammenfallende Repräsentant von $Q_{1}$ ist, ist notwendig und 
hinreichend aus dem Komplex der Elemente $Q_{1}$, für welche letzte $Q_{1}^{(k)} \equiv Q_{2}$ oder $\equiv Q_{3}$ ist und deren Anzahl, wie wir sahen, $2\left[2^{k}-(-1)^{k}\right]$ beträgt, diejenigen auszuscheiden, für welche schon $Q_{1}^{\left(k: p_{1}\right)}$, oder $Q_{1}^{\left(k: p_{2}\right)}, \ldots$ oder $Q_{1}^{\left(k: p_{s}\right)}$ mit einem der beiden Elemente $Q_{2}$ und $Q_{3}$ zusammenfällt.

Nunmehr zeigt eine zu der obigen (Nr. 31) ganz analoge Betrachtung, weil auch hier, wenn $Q_{1}^{(m)}$ und ebenso $Q_{1}^{(n)}$ mit einem der beiden Elemente $Q_{2}$ und $Q_{3}$ zusammenfällt, $Q_{1}^{(d)} \equiv Q_{2}$ oder $\equiv Q_{3}$ sein wird, wo $d$ der größte gemeinsame Teiler von $m$ und $n$ bedeutet (ist nämlich $Q_{1}^{(i)}$ der niedrigste mit einem der beiden Elemente $Q_{2}$ und $Q_{3}$ zusammenfallende Repräsentant von $Q_{1}$, so sind $m$ und $n$ und mithin auch $d$ Vielfache von $i$, nicht aber von $3 i)$, daß, wenn $k=3^{\pi} p_{1}^{\pi_{1}} p_{2}^{\pi_{2}} \ldots p_{s}^{\pi_{s}}\left(1<p_{1}<p_{2}<\cdots<p_{s}\right.$; $\pi \geqq 0, \pi_{1} \geqq 1, \pi_{2} \geqq 1, \ldots \pi_{s} \geqq 1$ ), wo $s>1$, oder wo zwar $s=1$, aber $p_{1}>3$, oder endlich wo $s=1, p_{1}=2$, aber $\pi_{1}>1$ ist, die Anzahl derjenigen Elemente $Q_{1}$, für welche $Q_{1}^{\left(k^{k}\right)}$ der niedrigste mit einem der beiden Elemente $Q_{2}$ und $Q_{3}$ zusammenfallende Repräsentant von $Q_{1}$ ist, gleich ist:

$$
2 \sum_{\alpha_{1}, \alpha_{2}, \ldots \alpha_{s}}(-1)^{\alpha_{1}+\alpha_{2}+\cdots+\alpha_{s}} 2^{k: p_{1}^{\alpha_{1}} p_{2}^{\alpha_{\vartheta}} \ldots p_{s}^{\alpha_{s}},}
$$

wo $\alpha_{1}, \alpha_{2}, \ldots \alpha_{s}$ unabhängig voneinander die Werte 0 und 1 annehmen sollen, und, nur wenn $s=1, p_{1}=2, \pi_{1}=1$, die nämliche Anzahl gleich ist:

$$
2\left[\sum_{a_{1}}(-1)^{\alpha_{1}} 2^{k: 2^{\alpha_{1}}}-2\right]
$$

wo $\alpha_{1}$ die Werte 0 und 1 annehmen soll. Die nämliche Anzahl ist also in allen Fällen, wenn nur $s>0$ ist, gleich:

3) $2\left[\sum_{\alpha_{1}, \alpha_{2}, \ldots \alpha_{s}}(-1)^{\alpha_{1}+\alpha_{2}+\cdots+\alpha_{s}} 2^{k: p_{1}^{\alpha_{1}} p_{2}^{\alpha_{2} \cdots} p_{s}^{\alpha_{s}}}+(-1)_{1}^{p_{1}^{s-2} \cdot k}-(-1)^{k}\right]$; da $(-1)^{p_{1}^{s-2 . k}}-(-1)^{k}=0$ ist $\left[\right.$ in $p_{1}^{s} \cdot k\left(=p_{1}^{s+\pi_{1}} \frac{k}{p_{1}^{\pi_{1}}}\right)$ geht $p_{1}^{2}$ auf, wenn nur $s>0, p_{1}^{s-2} . k$ ist also eine ganze Zahl], wenn $p_{1}>3$ und also $p_{1}$ und $k$ beide zugleich ungerade sind, ferner wenn $p_{1}=2$ und $\pi_{1} \geqq 2$, wo dann $k$ und $p_{1}^{s-2} \cdot k\left(=2^{\pi_{1}+s-2} \frac{k}{2^{\pi_{1}}}\right)$ beide zugleich gerade sind, und endlich wenn $p_{1}=2, \pi_{1}=1$ und $s \geqq 2$, wo dann wieder $k$ und $p_{1}^{s-2} \cdot k\left(=2^{s-2} k\right)$ beide zugleich 
gerade sind, und nur dann $(-1)^{p_{1}^{s}-2 . k}-(-1)^{k}=-2$ ist, wenn $p_{1}=2, \pi_{1}=s_{1}=1$ ist, wo dann $k$ gerade, während $p_{1}^{s-2} \cdot k\left(=2^{-1} k\right)$ ungerade ist. Ist aber $s=0$, ist also $k=3^{\pi}$, so ist die nämliche Anzahl gleich:

$$
2\left(2^{k}+1\right)
$$

denn, wäre für ein Element $Q_{1}$, für das $Q_{1}^{(k)}$ mit einem der beiden Elemente $Q_{2}$ und $Q_{3}$ zusammenfällt, $Q_{1}^{(i)}$ der niedrigste Repräsentant von $Q_{1}$, der mit einem der beiden Elemente $Q_{2}$ und $\zeta_{3}$ zusammenfällt, wo $i<k$, so müßte, wie wir sahen, $k$ ein Vielfaches von $i$ und kein Vielfaches von $3 i$ sein, was, wenn $k=3^{\pi}$ und $i<k$, unmöglich ist; es muß also in diesem Falle für alle $2\left[2^{k}-(-1)^{k}\right]=2\left(2^{k}+1\right)$ Elemente $Q_{1}$, für die $Q_{1}^{(k)}$ mit einem der beiden Elemente $Q_{2}$ und $Q_{3}$ zusammenfällt, $Q_{1}^{(k)}$ auch der niedrigste Repräsentant von $Q_{1}$ sein, der dies tut.

Ersetzt man in den Formeln 3) und 4) $k$ durch $\frac{n}{3}$, so ergibt sich:

Ist

$$
n=3^{\pi} p_{1}^{\pi_{1}} p_{2}^{\pi_{2}} \ldots p_{i}^{\pi_{i}}
$$

(wo $1<p_{1}<p_{2}<\cdots<p_{i} ; \pi \geqq 1, \pi_{1} \geqq 1, \pi_{2} \geqq 1, \ldots \pi_{i} \geqq 1 ; i \geqq 1$ ), so beträgt die Anzahl derjenigen Elemente $Q_{1}$, für die $Q_{1}^{(n: 3)}$ der niedrigste mit einem der beiden Elemente $Q_{2}$ und $Q_{3}$ zusammenfallende Repräsentant von $Q_{1}$ ist:

5) $2\left[\sum_{\alpha_{1}, \alpha_{2}, \ldots \alpha_{i}}(-1)^{\alpha_{1}+\alpha_{2}+\cdots+\alpha_{i}} 2^{n: 3} p_{1}^{\alpha_{1}} p_{2}^{\alpha_{2} \ldots p_{i}^{\alpha_{i}}}+(-1)^{p_{1}^{i-2} \cdot n}-(-1)^{n}\right]$, wo $\alpha_{1}, \alpha_{2}, \ldots \alpha_{i}$ unabhängig voneinander die Werte 0 und 1 annehmen sollen; ist aber $n=3^{\pi}(\pi \geqq 1)$, so beträgt die nämliche Anzahl:

$$
2\left(2^{n: 3}+1\right) \text {. }
$$

Die Formeln 5) und 6) liefern nun zugleich, wie im Anfang dieser Nummer bemerkt wurde, die Anzahl derjenigen primitiven $n$-ten Koinzidenz-Repräsentanten $Q_{1}$, unter deren Repräsentanten $Q_{2}$ und mithin auch $Q_{3}$ vorkommen, wenn $n=3^{\pi} p_{1}^{\pi_{1}} p_{2}^{\pi_{2}} \ldots p_{i}^{\pi} i$ ist bzw. wenn $n=3^{\pi}$ ist.

37. Weil nun die Formeln 5) und 6) (wo $\frac{n}{3}$ wiederum ein Vielfaches von 3 ist oder nicht), die die Anzahl der letzt erwähnten primitiven $n$-ten Koinzidenz-Repräsentanten liefern, je 
durch $n$ teilbar sein müssen (Ende Nr. 35), so ergibt sich, wenn man $\frac{n}{3}$ durch $m$ ersetzt und die Formeln 5) und 6) durch 2 bzw., wenn $i>1$ oder $p_{1}>3$ oder $\pi_{1}>1$ ist, die Formel 5) durch $(-1)^{i} 2^{\left(m: p_{1} p_{2} \ldots p_{i}\right)+1}$ dividiert, daß das Resultat in Nr. 32 wie folgt ergänzt werden kann:

Ist

$$
m=3^{\tau} p_{1}^{\pi_{1}} p_{2}^{\pi_{2}} \cdots p_{i}^{\pi_{i}}
$$

(wo $1<p_{1}<p_{2}<\cdots<p_{i} ; \pi \geqq 0, \pi_{1} \geqq 1, \pi_{2} \geqq 1, \ldots \pi_{i} \geqq 1 ; i \geqq 1$ ) und ist $i>1$, oder $p_{1}>3$, oder $\pi_{1}>1$, so ist, je nachdem $m$ ungerade oder gerade und also $p_{1}=2$ ist:

$$
\begin{aligned}
\sum_{\alpha_{1}, \alpha_{2}, \ldots \alpha_{i}}(-1)^{\alpha_{1}+\alpha_{2}+\cdots+\alpha_{i}} \cdot 2^{m\left(p_{1}^{\alpha_{1}} p_{2}^{\alpha_{2}} \ldots p_{i}^{\alpha_{i}}-1\right): p_{1} p_{2} \ldots p_{i}} \\
\equiv 0\left(\bmod .3 m \text { oder mod. } 3 m: 2^{\pi_{1}}\right),
\end{aligned}
$$

wo $\alpha_{1}, \alpha_{2}, \ldots \alpha_{i}$ unabhängig voneinander die Werte 0 und 1 annehmen sollen; ist aber $i=1, p_{1}=2$ und $\pi_{1}=1$, ist also

so ist:

$$
m=3^{\pi} \cdot 2 \quad(\pi \geqq 0),
$$

$$
\sum_{\alpha_{1}}(-1)^{\alpha_{1}} 2^{m: 2^{\alpha_{1}}} \equiv 2(\bmod .3 m)
$$

oder :

$$
\sum_{\alpha_{1}}(-1)^{\alpha_{1}} 2^{2^{1-\alpha_{1}} \cdot 3^{\pi}-1} \equiv 1\left(\bmod .3^{\pi+1}\right)
$$

wo $\alpha_{1}$ die Werte 0 und 1 annehmen soll; ist endlich

so ist:

$$
m=3^{\pi} \text {, }
$$

$$
2^{3^{\pi}} \equiv-1\left(\bmod .3^{\pi+1}\right)
$$

was auch leicht auf andere Weise bewiesen wird.

38. Ebenso wie wir den Repräsentanten einer Gruppe in der von $A B C$ im Gebilde erzeugten Involution $\left(2^{n}\right)$-ten Grades den $n$-ten Repräsentanten eines jeden der $2^{n}$ der nämlichen Gruppe angehörenden Elemente nannten (Nr.24), nennen wir das Repräsentations-Tripel (Nr. 20) einer Gruppe in der von $A B C$ im Gebilde erzeugten Involution ( $\left.2^{n} \cdot 3\right)$-ten Grades die $n$-te Repräsentation eines jeden der $2^{n}$ in dieser Gruppe enthaltenen Tripel der von $A B C$ im Gebilde erzeugten Involution dritten Grades (Satz 12 in Nr. 22). Die $n$-te Repräsentation eines Tripels der Involution dritten Grades besteht also (Nr. 20) aus demjenigen Tripel der- 
selben Involution, welches von den drei $n$-ten Repräsentanten der Elemente des erstern Tripels gebildet wird. In Analogie mit den $n$-ten Koinzidenz-Repräsentanten nennen wir ein Tripel, das mit seiner $n$-ten Repräsentation zusammenfällt, eine $n$-te Koinzidenz-Repräsentation und, wenn das Tripel erst mit seiner $n$-ten (und mit keiner frühern) Repräsentation zusammenfällt, eine primitive $n$-te Koinzidenz-Repräsentation.

Was die Anzahl anbetrifft, unterscheiden sich nicht die Koinzidenz-Repräsentationen von den Koinzidenz-Repräsentanten.

Es ist nämlich die Anzahl der $n$-ten Koinzidenz-Repräsentationen gleich:

$$
2^{n}-(-1)^{n}
$$

und die Anzahl der primitiven $n$-ten Koinzidenz-Repräsentationen gleich:

$$
\sum_{\alpha_{1}, \alpha_{2}, \ldots \alpha_{k}}(-1)^{\alpha_{1}+\alpha_{2}+\cdots+\alpha_{k}} 2^{n: p_{1}^{\alpha_{1}} p_{2}^{\alpha_{2}} \cdots p_{k}^{\alpha} k}
$$

(wo $\alpha_{1}, \alpha_{2}, \ldots \alpha_{k}$ unabhängig voneinander die Werte 0 und 1 annehmen sollen), wenn

$$
n=p_{1}^{\pi_{1}} p_{2}^{\pi_{2}} \ldots p_{k}^{\pi_{k}}>2
$$

ist, und gleich 0 , wenn $n=2$ ist.

Denn auf drei Arten kann ein Tripel $Q_{1} Q_{2} Q_{3}$ mit seiner $n$-ten Repräsentation zusammenfallen, nämlich: erstens, kann $Q_{1}^{(n)} \equiv Q_{1}$ und alsdann muß auch $Q_{2}^{(n)} \equiv Q_{2}$ und $Q_{3}^{(n)} \equiv Q_{3}$ sein, zweitens, kann $Q_{1}^{(n)} \equiv Q_{2}$ und alsdann muß $Q_{2}^{(n)} \equiv Q_{3}$ und $\chi_{3}^{(n)} \equiv Q_{1}$ sein, drittens, kann $Q_{1}^{(n)} \equiv Q_{3}$ und alsdann muß $Q_{2}^{(n)} \equiv Q_{1}$ und $Q_{3}^{(n)} \equiv Q_{2}$ sein (Satz 20 in Nr. 34). Nun beträgt die Anzahl der Elemente $Q_{1}$, für die $Q_{1}^{(n)} \equiv Q_{1}$ ist, $2^{n}-(-1)^{n}$ (Satz 19 in Nr. 29) und die Anzahl derjenigen Elemente $Q_{1}$, für die je $Q_{1}^{(n)}$ mit einem der beiden Elemente $Q_{2}$ und $Q_{3}$ zusammenfällt, $2\left[2^{n}-(-1)^{n}\right]$ (Nr. 36); mithin beträgt die Anzahl derjenigen Elemente $Q_{1}$, für die je $Q_{1}^{(n)}$ mit einem der Elemente des Tripels $Q_{1} Q_{2} Q_{3}$ zusammenfällt, $3\left[2^{n}-(-1)^{n}\right]$. Weil aber erst drei solche Elemente ein mit seiner $n$-ten Repräsentation zusammenfallendes Tripel ausmachen, so beträgt die Anzahl dieser Tripel, also die Anzahl der $n$-ten Koinzidenz-Repräsentationen $2^{n}-(-1)^{n}$.

Soll ferner $Q_{1} Q_{2} Q_{3}$ ein erst mit seiner $n$-ten (und mit keiner früheren) Repräsentation zusammenfallendes Tripel sein, 
so wird, wenn $Q_{1}^{(n)} \equiv Q_{1}$ sein soll, $Q_{1}$ irgendein primitiver $n$-ter Koinzidenz-Repräsentant sein müssen, wenn $n$ kein Vielfaches von 3 ist und also $Q_{2}$ und $Q_{3}$ nicht unter den Repräsentanten von $Q_{1}$ vorkommen können (Satz 21 in Nr. 34); ist aber $n \equiv 0$ (mod. 3), so wird dann $Q_{1}$ ein solcher primitiver $n$-ter Koinzidenz-Repräsentant sein müssen, unter dessen Repräsentanten weder $Q_{2}$ noch $Q_{3}$ vorkommt, da sonst $Q_{1}^{(n: 3)} \equiv Q_{2}$ oder $Q_{3}$ wäre (ebenda) und mithin $Q_{1} Q_{2} Q_{3}$ schon mit seiner $\left(\frac{n}{3}\right)$-ten Repräsentation zusammenfiele; und wenn $Q_{1}^{(n)} \equiv Q_{2}$ oder $\equiv Q_{3}$ sein soll, wird $Q_{1}$ ein solches Element sein müssen, für das $Q_{1}^{(n)}$ der niedrigste mit einem der beiden Elemente $Q_{2}$ und $Q_{3}$ zusammenfallende Repräsentant von $Q_{1}$ ist. In allen Fällen werden $Q_{2}$ und $Q_{3}$ ebenso wie $Q_{1}$ beschaffen sein müssen (Satz 20 in Nr. 34). Nun beträgt aber, wenn

$$
n=p_{1}^{\pi_{1}} p_{2}^{\pi_{2}} \ldots p_{k}^{\pi_{k}}>2
$$

und $n$ kein Vielfaches von 3 ist, die Anzahl derjenigen Elemente $Q_{1}$, welche primitive $n$-te Koinzidenz-Repräsentanten sind:

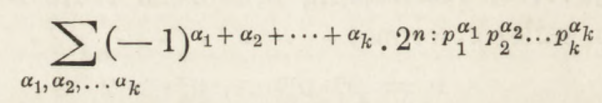

(Nr. 31) und die Anzahl derjenigen Elemente $Q_{1}$, für die je $Q_{1}^{(n)}$ der niedrigste mit einem der beiden Elemente $Q_{2}$ und $Q_{3}$ zusammenfallende Repräsentant von $Q_{1}$ ist:

$$
2 \sum_{\alpha_{1}, \alpha_{2}, \ldots \alpha_{k}}(-1)^{\alpha_{1}+\alpha_{2}+\cdots+\alpha_{k}} \cdot 2^{n: p_{1}^{\alpha_{1}}} p_{2}^{\alpha_{2} \ldots p_{k}^{\alpha}}
$$

[nach 1) in Nr. 36], also zusammen:

$$
3 \sum_{\alpha_{1}, \alpha_{2}, \ldots \alpha_{k}}(-1)^{\alpha_{1}+\alpha_{2}+\cdots+\alpha_{k}} \cdot 2^{n: p_{1}^{\alpha_{1}}} p_{2}^{\alpha_{2} \ldots p_{k}^{\alpha_{k}}} ;
$$

mithin beträgt dann die Anzahl der aus je drei solchen Elementen bestehenden Tripel, also die Anzahl der primitiven $n$-ten Koinzidenz-Repräsentationen:

$$
\sum_{\alpha_{1}, \alpha_{2}, \ldots \alpha_{k}}(-1)^{\alpha_{1}+\alpha_{2}+\cdots+\alpha_{k} .2^{n: p_{1}^{\alpha_{1}}} p_{2}^{\alpha_{2} \ldots p_{k}^{\alpha_{k}}} .}
$$

Wenn ferner $n \equiv 0$ (mod. 3 ), etwa $p_{1}=3$ und $k \geqq 2$ ist (wo dann $1<p_{2}<p_{3}<\cdots<p_{k}$ sein sollen), so beträgt die Anzahl derjenigen primitiven $n$-ten Koinzidenz-Repräsentanten, unter deren Repräsentanten je weder $Q_{2}$ noch $Q_{3}$ vorkommt: 


$$
\begin{aligned}
& \sum_{\alpha_{1}, \alpha_{2}, \ldots \alpha_{k}}(-1)^{\alpha_{1}+\alpha_{2}+\cdots+\alpha_{k} \cdot 2^{n: 3^{\alpha_{1}}} p_{2}^{\alpha_{2}} \ldots p_{k}^{\alpha_{k}}} \\
& -2\left[\sum_{\alpha_{2}, \ldots \alpha_{k}}(-1)^{\alpha_{2}+\cdots+\alpha_{k}} \cdot 2^{n: 3} p_{2}^{\alpha_{2}} \cdots p_{k}^{\alpha} k+(-1)^{p_{2}^{k-3} \cdot n}-(-1)^{n}\right]
\end{aligned}
$$

[nach Nr. 31 und 5) in Nr. 36; hier ist deshalb $p_{2}^{k-3} \cdot n$ der Exponent des vorletzten Gliedes in der eckigen Klammer, während in 5) $p_{1}^{i-2} \cdot n$ es ist, weil hier in den $k$ voneinander verschiedenen Primfaktoren von $n$ auch der Faktor 3 mitgezählt wird] und die Anzahl derjenigen Elemente $Q_{1}$, für die je $Q_{1}^{(n)}$ der niedrigste mit einem der beiden Elemente $Q_{2}$ und $Q_{3}$ zusammenfallende Repräsentant von $Q_{1}$ ist:

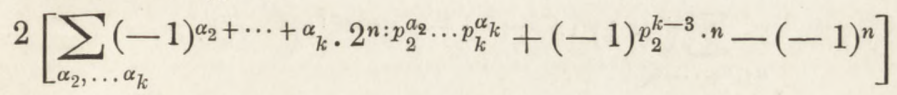

[nach 3) in Nr. 36], also zusammen:

$$
\begin{aligned}
& \sum_{\alpha_{1}, \alpha_{2}, \ldots \alpha_{k}}(-1)^{\alpha_{1}+\alpha_{2}+\cdots+\alpha_{k}} \cdot 2^{n: 3^{\alpha_{1}}} p_{2}^{\alpha_{2}} \ldots p_{k}^{\alpha} k \\
& -2 \sum_{\alpha_{2}, \ldots \alpha_{k}}(-1)^{\alpha_{2}+\cdots+\alpha_{k}} \cdot 2^{n: 3} p_{2}^{\alpha_{2}} \cdots p_{k}^{\alpha}
\end{aligned}
$$

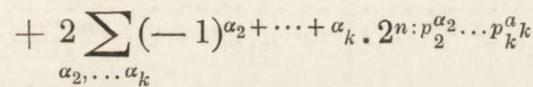

$$
\begin{aligned}
& =\sum_{\alpha_{1}, \alpha_{2}, \ldots \alpha_{k}}(-1)^{\alpha_{1}+\alpha_{2}+\cdots+\alpha_{k}} \cdot 2^{n: 3^{\alpha_{1}}} p_{2}^{\alpha_{2}} \ldots p_{k}^{\alpha_{k}} \\
& +2 \sum_{\alpha_{2}, \ldots \alpha_{k}}^{\alpha_{1}=1}(-1)^{\alpha_{1}+\alpha_{2}+\cdots+\alpha_{k}} \cdot 2^{n: 3^{\alpha_{1}}} p_{2}^{\alpha_{2}} \ldots p_{k}^{\alpha_{k}} \\
& +2 \sum_{\alpha_{2}, \ldots \alpha_{k}}^{\alpha_{1}=0}(-1)^{\alpha_{1}+\alpha_{2}+\cdots+\alpha_{k}} \cdot 2^{n: 3^{\alpha_{1}}} p_{2}^{\alpha_{2}} \ldots p_{k}^{\alpha_{k}} \\
& =\sum_{\alpha_{1}, \alpha_{2}, \ldots \alpha_{k}}(-1)^{\alpha_{1}+\alpha_{2}+\cdots+\alpha_{k} \cdot 2^{n: 3^{\alpha_{1}}} p_{2}^{\alpha_{2} \ldots p_{k}^{\alpha}}} \\
& +2 \sum_{\alpha_{1}, \alpha_{2}, \ldots \alpha_{k}}(-1)^{\alpha_{1}+\alpha_{2}+\cdots+\alpha_{k} \cdot 2^{n: 3^{\alpha_{1}}} p_{2}^{\alpha_{2} \ldots p_{k} \alpha_{k}}} \\
& =3 \sum_{\alpha_{1}, \alpha_{2}, \ldots \alpha_{k}}(-1)^{\alpha_{1}+\alpha_{2}+\cdots+\alpha_{k}} \cdot 2^{n: 3^{\alpha_{1}} p_{2}^{\alpha_{2}} \ldots p_{k}^{\alpha_{k}}}
\end{aligned}
$$

(wo $\alpha_{1}, \alpha_{2}, \ldots \alpha_{k}$ unabhängig voneinander die Werte 0 und 1 annehmen sollen); und, wenn $n \equiv 0(\bmod .3)$ und $k=1$, also $n=p_{1}^{\pi_{1}}$ $=3^{\pi_{1}}$ ist, beträgt die erstere Anzahl:

$$
\sum_{\alpha_{1}}(-1)^{\alpha_{1}} 2^{n: 3^{\alpha_{1}}}-2\left(2^{n: 3}+1\right)
$$


[nach Nr.31 und 6) in Nr. 36] und die letztere Anzahl:

$$
2\left(2^{n}+1\right)
$$

[nach 4) in Nr. 36], also zusammen:

$$
\begin{gathered}
\sum_{\alpha_{1}}(-1)^{\alpha_{1}} 2^{n: 3^{\alpha_{1}}}+2\left(2^{n}-2^{n: 3}\right)=\sum_{\alpha_{1}}(-1)^{\alpha_{1}} 2^{n: 3^{\alpha_{1}}} \\
\quad+2 \sum_{\alpha_{1}}(-1)^{\alpha_{1}} 2^{n: 3^{\alpha_{1}}}=3 \sum_{\alpha_{1}}(-1)^{\alpha_{1}} 2^{n: 3^{\alpha_{1}}}
\end{gathered}
$$

mithin beträgt auch in allen Fällen, wo $n \equiv 0$ (mod. 3$)$ ist, die Anzahl der aus je drei solchen Elementen bestehenden Tripel, also die Anzahl der primitiven $n$-ten Koinzidenz-Repräsentationen:

$$
\sum_{\alpha_{1}, \alpha_{2}, \ldots \alpha_{k}}(-1)^{\alpha_{1}+\alpha_{2}+\cdots+\alpha_{k}} 2^{n: 3^{\alpha_{1}}} p_{2}^{\alpha_{2} \ldots p_{k}^{\alpha_{k}}} \text {. }
$$

Ist aber $n=2$, so ist die Anzahl der primitiven zweiten Koinzidenz-Repräsentanten gleich 0 (Nr. 31) und ebenso die Anzahl derjenigen Elemente $Q_{1}$, für die je $Q_{1}^{(2)}$ der niedrigste mit einem der beiden Elemente $Q_{2}$ und $Q_{3}$ zusammenfallende Repräsentant von $Q_{1}$ ist, gleich

$$
2\left[\sum_{\alpha_{1}}(-1)^{\alpha_{1}} 2^{2: 2^{\alpha_{1}}}-2\right]=2\left[2^{2}-2-2\right]=0
$$

[nach 2) in Nr. 36]; mithin muß auch die Anzahl der primitiven zweiten Koinzidenz-Repräsentationen gleich 0 sein.

\section{§ 7.}

39. Wie wir sahen, gibt es in einem Gebilde erster Stufe unendlich viele Elemente, welche wiederkehren, wenn wir den Prozeß ihrer Repräsentanten-Bildung hinreichend weit fortsetzen und welche Elemente wir Koinzidenz-Repräsentanten nannten; denn es gibt ja $2^{n}-(-1)^{n} n$-te Koinzidenz-Repräsentanten für jeden beliebigen positiven ganzzahligen Wert von $n$.

Es fragt sich nun:

Gibt es auch im Gebilde solche Elemente, welche nie wiederkehren, wie weit auch wir den Prozeß ihrer Repräsentanten-Bildung fortsetzen mögen? und, wenn es deren gibt, welches sind diese?

Zum Teil werden diese Fragen schon durch den folgenden Satz beantwortet. 
Satz 22. Unter den $2^{n}$ Elementen einer Gruppe in der von $A B C$ im Gebilde erzeugten Involution $\left(2^{n}\right)$-ten Grades kann höchstens ein Koinzidenz-Repräsentant vorkommen.

In der Tat, kämen zwei Koinzidenz-Repräsentanten, etwa $Q_{n, i}$ und $Q_{n, k}$, vor und wäre der eine ein $p$-ter und der zweite ein $q$-ter, wo $p=q$ oder $p \neq q$, so müßten $Q_{n, i}$ und $Q_{n, k}$, weil jeder $l$-te Koinzidenz-Repräsentant zugleich ein $(m l)$-ter ist (Nr. 30), zugleich $(p q)$-te und auch $(p q r)$-te Koinzidenz-Repräsentanten sein für jeden beliebigen Wert von $r$; es wären daher $Q_{n, i}^{(p q r)}$ $\left(\equiv Q_{n, i}\right)$ und $Q_{n, k}^{(p q r)}\left(\equiv Q_{n, k}\right)$ voneinander verschieden auch für einen solchen Wert von $r$, für den $p q r>n$ ist, was aber unmöglich ist, da $Q_{n, i}$ und $Q_{n, k}$, welche beide einer und derselben Gruppe in der Involution $\left(2^{n}\right)$-ten Grades angehören, den $n$-ten Repräsentanten, der der Repräsentant jener ganzen Gruppe ist (Nr. 24), und mithin auch jeden höhern als den $n$-ten Repräsentanten gemein haben müssen.

Wenn daher ein Element in einer Involution $\left(2^{k}\right)$-ten Grades (für irgendeinen Wert von $k$ ) einer solchen Gruppe angehört, die unter ihren $2^{n}$ Elementen schon einen Koinzidenz-Repräsentanten enthält, so kann das Element kein Koinzidenz-Repräsentant sein und also nie wiederkehren, wie weit auch wir den Prozeß seiner Repräsentanten-Bildung fortsetzen mögen.

40. Gehört aber ein Element in der Involution $\left(2^{k}\right)$-ten Grades einer einen Koinzidenz-Repräsentanten schon enthaltenden Gruppe an, so muß es auch in jeder höhern Involution, etwa vom $\left(2^{k+i}\right)$-ten Grade (für jeden Wert von $i$ ), einer ebensolchen Gruppe angehören; denn jede Gruppe in der letztern Involution ist aus $2^{i}$ Gruppen der erstern zusammengesetzt (Satz 12 in Nr. 22), mithin müssen das Element und der Koinzidenz-Repräsentant auch in der Involution $\left(2^{k+i}\right)$-ten Grades in einer und derselben Gruppe vorkommen.

Ist nun ein Element gegeben, das in der Involution $\left(2^{k}\right)$-ten Grades einer einen Koinzidenz-Repräsentanten schon enthaltenden Gruppe angehört, und ist in dieser Gruppe der KoinzidenzRepräsentant mit $Q_{k, r}$ und das gegebene Element mit $Q_{k, s}$ bezeichnet, wo die Ordnung der Elementenzeiger in dieser Gruppe mit der natürlichen Anordnung in einem der beiden Sinne im Gebilde übereinstimmt, und ist endlich $r-s \equiv 2^{i}\left(\bmod .2^{i+1}\right)$, wo 
notwendig $0 \leqq i<k$, so ist die Involution $\left(2^{k-i}\right)$-ten Grades die niedrigste, in der das gegebene Element einer einen KoinzidenzRepräsentanten schon enthaltenden Gruppe angehört. Denn, weil $r \equiv s\left(\bmod .2^{i}\right)$, gehören $Q_{k, r}$ und $Q_{k, s}$ auch in der Involution $\left(2^{k-i}\right)$-ten Grades einer und derselben Gruppe an (Satz 12 in Nr. 22); sollte aber $Q_{k, s}$ schon in einer Involution $\left(2^{k-l}\right)$-ten Grades $(i<l<k)$ einer solchen Gruppe angehören, die einen KoinzidenzRepräsentanten enthält, so würde dieser Koinzidenz-Repräsentant, welcher, weil $r \equiv s\left(\bmod .2^{l}\right)$ ist, von $Q_{k, r}$ verschieden sein müßte, mit $Q_{k, s}$ auch in der höhern Involution $\left(2^{k}\right)$-ten Grades in einer und derselben Gruppe vorkommen und mithin kämen in der letzten Gruppe zwei Koinzidenz-Repräsentanten, nämlich dieser und $Q_{k, r}$ vor, was nach Satz 22 unmöglich ist.

Ist die Involution $\left(2^{m}\right)$-ten Grades die niedrigste, in der ein gegebenes Element einer einen Koinzidenz-Repräsentanten schon enthaltenden Gruppe angehört, so ist die Involution $\left(2^{m-p}\right)$-ten Grades $(0<p<m)$ die niedrigste, in der der $p$-te Repräsentant des gegebenen Elementes gleichfalls einer einen KoinzidenzRepräsentanten schon enthaltenden Gruppe angehört. Es kann also der $p$-te Repräsentant des gegebenen Elementes (für $p<m$ ) ebenso wie dieses Element selbst kein Koinzidenz-Repräsentant sein; hingegen ist der $m$-te und mithin auch jeder noch höhere Repräsentant des gegebenen Elementes je ein Koinzidenz-Repräsentant, und zwar je ein primitiver $n$-ter, wenn der mit dem gegebenen Elemente in einer und derselben Gruppe der Involution $\left(2^{m}\right)$-ten Grades vorkommende Koinzidenz-Repräsentant ein primitiver $n$-ter ist. Seien nämlich der letzt erwähnte Koinzidenz-Repräsentant und das gegebene Element in der Gruppe der Involution $\left(2^{m}\right)$-ten Grades mit $Q_{m, r}$ und $Q_{m, s}$ bezeichnet, so müssen, weil (für jedes $p<m) Q_{m, r}$ und $Q_{m, s}$ zwei voneinander verschiedenen Gruppen der Involution $\left(2^{p}\right)$-ten Grades angehören, ihre $p$-ten Repräsentanten $Q_{(m, r)}^{(p)}$ und $Q_{(m, s)}^{(p)}$, die die Repräsentanten dieser zwei Gruppen der Involution $\left(2^{p}\right)$-ten Grades sind (Nr. 24) und wo $Q_{(m, r)}^{(p)}$ ebenso wie $Q_{m, r}$ ein Koinzidenz-Repräsentant ist (Nr.30), voneinander verschieden sein und einer und derselben Gruppe, nämlich der Repräsentations-Gruppe von der $Q_{m, r}$ und $Q_{m, s}$ enthaltenden Gruppe der Involution $\left(2^{m}\right)$-ten Grades in der Involution $\left(2^{m-p}\right)$-ten Grades angehören (Nr. 19). $Q_{(m, r)}^{(p)}$ und $Q_{(m, s)}^{(p)}$ können aber in einer niedrigern Involution, etwa vom $\left(2^{m-p-i}\right)$-ten Grade, nicht einer und 
derselben Gruppe angehören, da sonst $Q_{m, r}$ und $Q_{m, s}$ gegen die Voraussetzung schon in der Involution $\left(2^{m-i}\right)$-ten Grades einer und derselben Gruppe angehören müßten (nach Nr. 19). Sollte nun $Q_{(m, s)}^{(p)}$ schon in einer Involution $\left(2^{m-p-i}\right)$-ten Grades einer einen Koinzidenz-Repräsentanten enthaltenden Gruppe angehören, so müßte dieser Koinzidenz-Repräsentant, der nach dem soeben Bewiesenen von $Q_{(m, r)}^{(p)}$ verschieden wäre, zugleich mit $Q_{(m, r)}^{(p)}$, der gleichfalls ein Koinzidenz-Repräsentant ist, in der Involution $\left(2^{m-p}\right)$-ten Grades in einer und derselben Gruppe, nämlich in der $Q_{(m, s)}^{(p)}$ enthaltenden Gruppe, vorkommen, was aber nach Satz 22 unmöglich ist. Der $m$-te Repräsentant von $Q_{m, s}$, der der Repräsentant der ganzen $Q_{m, s}$ enthaltenden Gruppe der Involution $\left(2^{m}\right)$-ten Grades und also der nämliche wie der $m$-te von $Q_{m, r}$ ist, muß aber ebenso wie $Q_{m, r}$ ein primitiver $n$-ter KoinzidenzRepräsentant sein (Nr. 30).

Ist, umgekehrt, der $m$-te (und kein niedriger) Repräsentant eines gegebenen Elementes ein Koinzidenz-Repräsentant, so ist die Involution $\left(2^{m}\right)$-ten Grades die niedrigste, in der das gegebene Element einer solchen Gruppe angehört, die einen Koinzidenz-Repräsentanten schon enthält; und zwar ist dieser letzte Koinzidenz-Repräsentant ein primitiver $n$-ter, wenn der $m$-te Repräsentant des gegebenen Elementes es ist. Sei nämlich das gegebene Element mit $Q$ bezeichnet und $m=q n+r(0 \leqq r<n)$, so

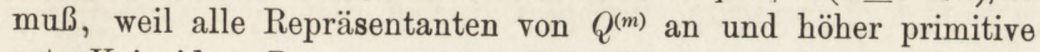
$n$-te Koinzidenz-Repräsentanten sind, der $m$-te Repräsentant von $Q^{(m+n-r)}$, also $Q^{(m+n-r)(m)} \equiv Q^{(m+n-r)(q n+r)} \equiv Q^{(m-r)(n)(r)(q n)}$ $\equiv Q^{(m-r+r)(q n+n)} \equiv Q^{(m)(q n+n)} \equiv Q^{(m)}$ sein, wo $Q^{(m+n-r)}$, weil $r<n$ und also $m+n-r>m$ ist, ein primitiver $n$-ter KoinzidenzRepräsentant sein muß. Mithin gehört $Q$ in der Involution $\left(2^{m}\right)$-ten Grades einer solchen Gruppe an, die schon den primitiven $n$-ten Koinzidenz-Repräsentanten $Q^{(m+n-r)}$ enthält; da $Q$ und $Q^{(m+n-r)}$ beide einen und denselben $m$-ten Repräsentanten, nämlich $Q^{(m)}$, haben (Nr. 24). Sollte aber $Q$ schon in einer Involution $\left(2^{l}\right.$ )-ten Grades (wo $l<m$ ) einer solchen Gruppe angehören, die einen Koinzidenz-Repräsentanten enthält, so müßte, wie wir sahen, gegen die Voraussetzung schon der $l$-te Repräsentant von $Q$ ein Koinzidenz-Repräsentant sein.

Wenn daher ein Element $Q$ in einer Involution $\left(2^{k}\right)$-ten Grades, wie groß auch $k$ genommen werden mag, einer keinen Berliner, Habilitationsschrift. 
Koinzidenz-Repräsentanten enthaltenden Gruppe angehört, so kann weder $Q$ selbst, noch irgendeiner seiner Repräsentanten $Q^{(l)}$ für irgendeinen Wert von $l$ ein Koinzidenz-Repräsentant sein und also wie weit auch wir den Prozeß der RepräsentantenBildung von $Q$ fortsetzen mögen, wird sich ein und derselbe Repräsentant von $Q$ nie zweimal ergeben, da sonst ein solcher Repräsentant ein Koinzidenz-Repräsentant sein müßte.

41. Bis nun haben wir uns nur von der Existenz zweier Arten von Elementen überzeugt, nämlich von Elementen, die selbst Koinzidenz-Repräsentanten sind, und von Elementen, die zwar selbst keine Koinzidenz-Repräsentanten sind, doch von solchen repräsentiert werden, welche letztere Elemente also je in den Involutionen von irgendeinem Grade $2^{m}$ an solchen Gruppen angehören, die schon je einen Koinzidenz-Repräsentanten enthalten. Wir werden aber später (Nr. 42) sehen, daß auch die dritte Art von Elementen wirklich existiert, welche letzte Elemente weder selbst Koinzidenz-Repräsentanten sind, noch von solchen repräsentiert werden und welche Elemente also in keiner Involution, wie groß auch deren Grad $2^{k}$ genommen werden mag, einer einen Koinzidenz-Repräsentanten enthaltenden Gruppe angehören.

Hiernach ergibt sich, wenn wir als den nullten Repräsentanten eines Elementes das Element selbst ansehen und daher den Prozeß der Repräsentanten-Bildung für ein Element als von diesem Element an beginnend betrachten:

Hinsichtlich des Prozesses der Repräsentanten-Bildung zerfallen die Elemente im Gebilde erster Stufe in drei Klassen:

Die erste Klasse enthält alle die Elemente, welche selbst Koinzidenz-Repräsentanten sind. Für ein Element $Q$ dieser Klasse ist der Prozeß seiner Repräsentanten-Bildung rein periodisch: die Periode beginnt nämlich gleich bei $Q$ und ist $n$-gliedrig, wenn $Q$ ein primitiver $n$-ter KoinzidenzRepräsentant ist; wie weit auch wir den Prozeß der Repräsentanten-Bildung dann fortsetzen mögen, wird sich nur die $n$-gliedrige Periode $Q, Q^{(1)}, \ldots Q^{(n-1)}$ unaufhörlich wiederholen, nie aber wird sich ein anderes Element ergeben.

Die zweite Klasse enthält alle die Elemente, welche zwar selbst keine Koinzidenz-Repräsentanten sind, doch mit 
solchen in je einer und derselben Gruppe in den von $A B C$ im Gebilde erzeugten Involutionen (von einem gewissen Grade an und höher) vorkommen. Für ein Element $Q$ dieser Klasse ist der Prozeß seiner Repräsentanten-Bildung gemischt periodisch: die Periode beginnt erst beim $m$-ten Repräsentanten $Q^{(m)}$ von $Q$, wenn die Involution $\left(2^{m}\right)$-ten Grades die niedrigste ist, in der $Q$ einer einen KoinzidenzRepräsentanten enthaltenden Gruppe angehört, und ist $n$-gliedrig, wenn der soeben erwähnte Koinzidenz-Repräsentant ein primitiver $n$-ter ist; wie weit auch wir dann den Prozeß der Repräsentanten-Bildung fortsetzen mögen, werden $Q, Q^{(1)}, \ldots Q^{(m-1)}$ nur je einmal vorkommen und darauf wird sich die $n$-gliedrige Periode $Q^{(m)}, Q^{(m+1)}, \ldots Q^{(m+n-1)}$ unaufhörlich wiederholen, nie aber wird sich außer diesen ein anderes Element ergeben.

Endlich enthält die dritte Klasse alle die Elemente, die weder selbst Koinzidenz-Repräsentanten sind, noch mit solchen in einer und derselben Gruppe in irgendeiner der von $A B C$ im Gebilde erzeugten Involutionen vorkommen, wie groß auch der Grad $2^{k}$ der Involution genommen werden mag. Für ein Element dieser Klasse werden sich, wie weit auch wir den Prozeß der Repräsentanten-Bildung dann fortsetzen mögen, immer neue und neue Elemente ergeben, nie aber wird ein und dasselbe Element zweimal vorkommen.

Die Elemente einer jeden dieser drei Klassen liegen im Gebilde überall dicht.

Denn in einem noch so kleinen Intervalle liegen immer zwei aufeinanderfolgende Elemente einer Gruppe der Involution $\left(2^{k}\right)$-ten Grades, wenn $k$ hinreichend groß genommen wird (nach Nr. 26), und mithin auch der zwischen zwei solchen Elementen liegende $k$-te Koinzidenz-Repräsentant (Satz 19 in Nr.29), welcher ein Element der ersten Klasse ist, und nunmehr auch das auf diesen in der (diesen enthaltenden) Gruppe der Involution $\left(2^{k}\right)$-ten Grades folgende Element, welches letzte der zweiten Klasse angehört. Es liegen also im Gebilde die Elemente der ersten Klasse und ebenso die der zweiten überall dicht; es gilt aber dasselbe, wie wir bald sehen werden, auch von den Elementen der dritten Klasse. 
42. Um nun über die Klasse eines Elementes $Q$ ) zu entscheiden, haben wir nur den Ordinatenwinkel $\omega=\alpha \pi$ von $Q \mathrm{zu}$ betrachten, wo $0 \leqq \omega<\pi$ (dies Intervall enthält nämlich die Ordinatenwinkel sämtlicher Elemente des Gebildes), also $0 \leqq \alpha<1$.

Ist, erstens, $\alpha$ rational, also $\alpha=\frac{i}{k}$ (wo $i<k$ und $i$ eine zu $k$ relative Primzahl ist) und, zweitens, der Neniner $k$ ungerade, so gehört dann und nur dann das Element $Q$ der ersten Klasse an. Gehört dann die Zahl 2 zu dem Exponenten $\delta$ in bezug auf den Modul $k$, so ist $Q$ ein primitiver $\left(\frac{\delta}{2}\right)$-ter, oder $\delta$-ter, oder endlich $(2 \delta)$-ter KoinzidenzRepräsentant und dem entsprechend die Periode seiner Repräsentanten-Bildung $\left(\frac{\delta}{2}\right)$-, oder $\delta$-, oder endlich $(2 \delta)$ gliedrig, je nachdem $\delta \equiv 2(\bmod .4)$ und $2^{\delta: 2} \equiv-1(\bmod k)$, oder $\delta \equiv 0(\bmod .4)$ oder zwar $\delta \equiv 2(\bmod .4)$, aber $2^{\delta: 2} \not \equiv-1$ (mod. $k$ ), oder endlich $\delta$ ungerade ist.

Ist aber der Nenner $k$ des reduzierten echten Bruches $\alpha=\frac{i}{k}$ gerade, so gehört dann und nur dann das Element $Q$ der zweiten Klasse an. Ist dann $k \equiv 2^{m}\left(\bmod .2^{m+1}\right)$ und gehört die Zahl $2 \mathrm{zu}$ dem Exponenten $\delta$ in bezug auf den Modul $\left(k: 2^{m}\right)$, so ist die Involution $\left(2^{m}\right)$-ten Grades die niedrigste, in der $Q$ einer solchen Gruppe angehört, die schon einen Koinzidenz-Repräsentanten enthält, und zwar einen primitiven $\left(\frac{\delta}{2}\right)$-ten, oder $\delta$-ten, oder endlich $(2 \delta)$-ten, je nachdem welcher der vorigen Fälle der Beschaffenheit von $\delta$ eintritt, und dem entsprechend ist dann $m$ die Anzahl der Elemente, die beim Prozesse der Repräsentanten-Bildung von $Q$ der Periode vorangehen, und $\frac{\delta}{2}$, oder $\delta$, oder endlich $2 \delta$ die Gliederanzahl der Periode.

Ist endlich $\alpha$ irrational, so gehört dann und nur dann das Element $Q$ der dritten Klasse an.

Denn $Q$ ist nur dann ein Koinzidenz-Repräsentant, wenn sein Ordinatenwinkel auf die Form $\frac{r \pi}{2^{s}-(-1)^{s}}$ gebracht werden 
kann, also nur dann, wenn $\alpha$ rational und der Nenner $k$ des reduzierten Bruches $\alpha=\frac{i}{k}$ ungerade ist; und zwar ist dann $Q$ ein primitiver $n$-ter, wenn $n$ der kleinste Exponent ist, für welchen eine Kongruenz von der Gestalt:

1)

$$
2^{n} \equiv(-1)^{n}(\bmod . k)
$$

möglich ist (aus $\frac{i \pi}{k}=\frac{r \pi}{2^{n}-(-1)^{n}}$ folgt nämlich $i\left[2^{n}-(-1)^{n}\right]$ $=r k$ und hieraus, weil $i$ und $k$ relative Primzahlen sind: $\left.2^{n} \equiv(-1)^{n}(\bmod . k)\right)$. Ist nun $\delta$ der Exponent, zu dem die Zahl 2 in bezug auf den Modul $k$ gehört, ist also $\delta$ der kleinste Exponent, für welchen eine Kongruenz von der Gestalt:

$$
2^{\delta} \equiv 1(\bmod . k)
$$

möglich ist, so gibt es keinen Exponenten $\gamma$, für welchen die Kongruenz

$$
2^{\gamma} \equiv-1(\bmod . k)
$$

möglich wäre, wenn $\delta$ ungerade ist; ist aber $\delta$ gerade und gibt es Exponenten, für welche eine Kongruenz von der Gestalt 3) möglich ist, so ist $\frac{\delta}{2}$ der kleinste unter diesen Exponenten (denn $\gamma$ kann kein Vielfaches von $\delta$ sein, ist nun $\gamma=q \delta+r$, wo $0<r<\delta$, so folgt aus $2^{q \delta}+r \equiv-1 \equiv-2^{q \delta}$, daß dann auch $2^{r} \equiv-1$ und hieraus: $2^{2 r} \equiv 1$, mithin muß $2 r$ ein Vielfaches von $\delta>r$ sein, also muß dann $r=\frac{\delta}{2}$ sein, was nur, wenn $\delta$ gerade ist, möglich ist). Ist also $\delta \equiv 2(\bmod .4)$, also $\delta$ gerade und $\frac{\delta}{2}$ ungerade und ist $2^{\delta: 2} \equiv-1$, also $2^{\delta: 2} \equiv(-1)^{\delta: 2}(\bmod . k)$, so ist $\frac{\delta}{2}$ der kleinste Exponent, für welchen eine Kongruenz von der Gestalt 1) möglich ist. Ist aber $\delta \equiv 0$ (mod. 4$)$, also auch $\frac{\delta}{2}$ gerade, so ist dann $\delta$ der kleinste Exponent, für welchen eine Kongruenz von der Gestalt 1) möglich ist; dasselbe ist der Fall, wenn zwar $\delta \equiv 2$ (mod.4), aber $2^{\delta: 2} \not \equiv-1$ (mod. $k$ ) ist. Ist endlich $\delta$ ungerade, so ist eine Kongruenz von der Gestalt 3) unmöglich und $2 \delta$ ist dann der kleinste Exponent, für den eine Kongruenz von der Gestalt 1) möglich ist. 
Wenn aber $k \equiv 2^{m}\left(\bmod .2^{m+1}\right)$ ist, so kann erst der Ordinatenwinkel des $m$-ten Repräsentanten $Q^{(m)}$ von $Q$, welcher Winkel $=(-2)^{m} \frac{i}{k} \pi=(-1)^{m} \frac{i}{k: 2^{m}} \pi$ ist (Satz 15 in Nr. 26) (wo nunmehr der Nenner $\left(k: 2^{m}\right)$ ungerade ist), auf die Form $\frac{r \pi}{2^{s}-(-1)^{s}}$ gebracht werden; es ist also dann erst $Q^{(m)}$ ein KoinzidenzRepräsentant und mithin (Nr.40) die Involution $\left(2^{m}\right)$-ten Grades die niedrigste, in der $Q$ einer einen Koinzidenz-Repräsentanten enthaltenden Gruppe angehört.

Wenn endlich $\alpha$ irrational ist, so kann weder der Ordinatenwinkel $\alpha \pi$ von $Q$, noch der Ordinatenwinkel $(-2)^{l} \alpha \pi$ von $Q^{(l)}$ (für irgendeinen Wert von $l$ ) auf die Form $\frac{r \pi}{2^{s}-(-1)^{s}}$ gebracht werden.

Zugleich sehen wir, daß auch die Elemente der dritten Klasse im Gebilde überall dicht liegen, da der Bereich der irrationalen Zahlen überall dicht ist.

Hiermit ist auch die im Anfang dieses Paragraphen gestellte Frage vollständig beantwortet.

\section{Hieraus ergibt sich:}

Durchläuft $k$ alle die Zahlen, welche der Forderung genügen, daß in bezug auf sie, als Moduln, eine gegebene Zahl $n=p_{1}^{\pi_{1}} p_{2}^{\pi_{2}} \ldots p_{i}^{\pi_{i}}>2$

der kleinste Exponent ist, für welchen eine Kongruenz von der Gestalt

$$
2^{n} \equiv(-1)^{n}(\bmod . k)
$$

möglich ist, so ist die entsprechende Summe

$$
\sum_{k} \varphi(k)=\sum_{\alpha_{1}, \alpha_{2}, \cdots \alpha_{i}}(-1)^{\alpha_{1}+\alpha_{2}+\cdots+\alpha_{i}} 2^{n: p_{1}^{\alpha_{1}} p_{2}^{\alpha_{2}} \cdots p_{i}^{\alpha_{i}},}
$$

wo $\varphi(k)$ die bekannte zahlentheoretische Funktion bedeutet und wo $\alpha_{1}, \alpha_{2}, \ldots \alpha_{i}$ unabhängig voneinander die Werte 0 und 1 annehmen sollen.

Denn, wie wir sahen, ist ein Element $Q$ nur dann ein primitiver $n$-ter Koinzidenz-Repräsentant, wenn der Nenner $k_{1}$ des reduzierten echten Bruches $\frac{i_{1}}{k_{1}}$ (wo $\frac{i_{1}}{k_{1}} \pi$ der Ordinatenwinkel von $Q$ 
ist) eine solche Zahl ist, welche der Forderung des vorstehenden Satzes genügt. Es liefern also die $\varphi\left(k_{1}\right)$ voneinander verschiedenen reduzierten echten Brüche, welche den nämlichen Nenner $k_{1}$ haben, $\varphi\left(k_{1}\right)$ voneinander verschiedene primitive $n$-te Koinzidenz-Repräsentanten. In gleicher Weise liefert aber jede andere Zahl $k_{2}$, welche ebenso wie $k_{1}$ derselben Forderung genügt, $\varphi\left(k_{2}\right)$ voneinander und von den $\varphi\left(k_{1}\right)$ vorigen verschiedene primitive $n$-te Koinzidenz-Repräsentanten, da die reduzierten Brüche $\frac{i_{1}}{k_{1}}$ und $\frac{i_{2}}{k_{2}}$ voneinander verschieden sein müssen, wenn $k_{1} \neq k_{2}$ ist. Mithin muß $\sum_{k} \varphi(k)$ der Anzahl aller primitiven $n$-ten KoinzidenzRepräsentanten gleich sein, diese Anzahl aber wird durch die Summe rechts geliefert (Nr.31).

Ist $n \equiv 0$ (mod. 4), so geht der vorstehende Satz (nach Nr. 42) in folgenden über:

Durchläuft $k$ alle die Zahlen, welche der Forderung genügen, daß in bezug auf sie, als Moduln, die Zahl 2 zu dem Exponenten $n$ gehört, so ist die entsprechende Summe

$$
\sum_{k} \varphi(k)=\sum_{\alpha_{1}, \alpha_{2}, \ldots \alpha_{i}}(-1)^{\alpha_{1}+\alpha_{2}+\cdots+\alpha_{i}} 2^{n: p_{1}^{\alpha_{1}}} p_{2}^{\alpha_{2}} \ldots p_{i}^{\alpha_{i}} .
$$

44. Die Elemente der ersten Klasse und ebenso die der zweiten lassen sich ihrerseits wieder in je zwei Arten einteilen.

Der ersten Art der ersten Klasse soll jeder solche Koinzidenz-Repräsentant $Q_{1}$ angehören, unter dessen Repräsentanten $Q_{2}$ und $Q_{3}$ vorkommen, wo $Q_{1} Q_{2} Q_{3}$ ein Tripel der von $A B C$ im Gebilde erzeugten Involution dritten Grades ist; und zwar muß $Q_{1}$ ein primitiver $(3 k)$-ter KoinzidenzRepräsentant sein, wenn $Q_{1}^{(k)}$ der niedrigste mit einem der beiden Elemente $Q_{2}$ und $Q_{3}$ zuşammenfallende Repräsentant von $Q_{1}$ ist (Satz 20 in Nr. 34). Hingegen soll der zweiten Art der ersten Klasse jeder solche Koinzidenz-Repräsentant $Q_{1}$ angehören, unter dessen Repräsentanten weder $Q_{2}$ noch $Q_{3}$ vorkommt.

Ferner soll der ersten Art der zweiten Klasse jedes solche Element $Q_{1}$ angehören, unter dessen Repräsentanten zwar weder $Q_{2}$ noch $Q_{3}$, doch Repräsentanten von $Q_{2}$ und $Q_{3}$ vor- 
kommen, welches Element $Q_{1}$ also mit einem KoinzidenzRepräsentanten erster Art in einer und derselben Gruppe in den von $A B C$ im Gebilde erzeugten Involutionen (von einem gewissen Grade an und höher) vorkommt; und zwar ist die Involution $\left(2^{m}\right)$-ten Grades die niedrigste, in der $Q_{1}$ mit dem Koinzidenz-Repräsentanten in einer und derselben Gruppe vorkommt, und dieser Koinzidenz-Repräsentant ein primitiver $3(n-m)$ - ter, wenn $Q_{1}^{(m)}$ der niedrigste Repräsentant von $Q_{1}$ ist, welcher mit einem der Repräsentanten von $Q_{2}$ und $Q_{3}$ zusammenfällt, und wenn dieser Repräsentant von $Q_{2}$ oder $Q_{3}$ der $n$-te ist, wo notwendig $n>m$ ist. Hingegen soll der zweiten Art der zweiten Klasse jedes solche Element $Q_{1}$ derselben Klasse angehören, unter dessen Repräsentanten weder Repräsentanten von $Q_{2}$ noch von $Q_{3}$ vorkommen, welches Element $Q_{1}$ also mit einem KoinzidenzRepräsentanten zweiter Art in einer und derselben Gruppe vorkommt.

Ist nämlich $Q_{1}^{(m)} \equiv Q_{2}^{(n)}$, so muß auch $Q_{2}^{(m)} \equiv Q_{3}^{(n)}$ und $Q_{3}^{(m)} \equiv Q_{1}^{(n)}$ sein, da $Q_{1}^{(m)} Q_{2}^{(m)} Q_{3}^{(m)}$ und $Q_{1}^{(n)} Q_{2}^{(n)} Q_{3}^{(n)}$ zwei gleichen Sinn habende Tripel in der Involution dritten Grades bilden (Nr.33), wo $n>m$ sein $m u ß$, da sonst gegen die Voraussetzung schon ein niedriger als der $m$-te Repräsentant von $Q_{1}$, nämlich $Q_{1}^{(n)}$, mit $Q_{3}^{(m)}$ zusammenfiele, und wo $Q_{1}^{(n)} \equiv Q_{1}^{(m+n-m)} \equiv Q_{1}^{(m)(n-m)}$ der niedrigste mit einem der beiden Elemente $Q_{2}^{(m)}$ und $Q_{3}^{(m)}$ zusammenfallende Repräsentant von $Q_{1}^{(m)}$ sein muß; denn wäre schon $Q_{1}^{(l)} \equiv Q_{2}^{(m)}$ oder $\equiv Q_{3}^{(m)}$, wo $m<l<n$, so müßte gegen die Voraussetzung auch $Q_{1}^{(m)} \equiv Q_{3}^{(l)}$ bzw. $Q_{2}^{(l)}$ sein. Mithin muß $Q_{1}^{(m)}$ ein primitiver $3(n-m)$-ter Koinzidenz-Repräsentant erster Art sein (Satz 20 in Nr. 34). Sollte schon $Q_{1}^{(k)}$ und mithin auch $Q_{2}^{(k)}$ (Satz 20) je ein KoinzidenzRepräsentant sein, wo $k<m$, so müßte (nach $\mathrm{Nr}$.35) $Q_{2}^{(k+n-m)}$ $\equiv Q_{2}^{(k)(n-m)} \equiv Q_{1}^{(k)}$ sein, ebenso wie $Q_{2}^{(m)(n-m)} \equiv Q_{2}^{(m+n-m)} \equiv Q_{2}^{(n)} \equiv Q_{1}^{(m)}$ ist, da $Q_{2}^{(m)} \equiv Q_{2}^{(k+m-k)} \equiv Q_{2}^{(k)(m-k)}$ der $(m-k)$-te Repräsentant des (nach Annahme) Koinzidenz-Repräsentanten $Q_{2}^{(k)}$ wäre; was aber gegen die Voraussetzung, daß erst $Q_{1}^{(m)}$ mit einem der Repräsentanten von $Q_{2}$ und $Q_{3}$ zusammenfällt, wäre. Es ist also $Q_{1}^{(m)}$ der niedrigste Repräsentant von $Q_{1}$, welcher ein KoinzidenzRepräsentant ist; mithin ist dann die Involution $\left(2^{m}\right)$-ten Grades die niedrigste, in der $Q_{1}$ mit einem Koinzidenz-Repräsentanten in 
einer und derselben Gruppe vorkommt (Nr.40), und zwar muß dieser Koinzidenz-Repräsentant, der denselben $m$-ten Repräsentanten wie $Q_{1}$ hat, ebenso wie $Q_{1}^{(m)}$ ein primitiver $3(n-m)$-ter Koinzidenz-Repräsentant erster Art sein (nach Nr. 35). Ist, umgekehrt, der Koinzidenz-Repräsentant, welcher mit $Q_{1}$ in einer und derselben Gruppe vorkommt, von der ersten Art, so müssen auch alle seine Repräsentanten, also auch die, welche ihm und $Q_{1}$ gemein sind, Koinzidenz-Repräsentanten erster Art sein (Nr. 35); ist nun etwa $Q_{1}^{(r)}$ ein gemeinsamer Repräsentant von $Q_{1}$ und jenem Koinzidenz-Repräsentanten, so müssen, weil $Q_{1}^{(r)} Q_{2}^{(r)} Q_{3}^{(r)}$ ein Tripel der Involution dritten Grades ist, $Q_{2}^{(r)}$ und $Q_{3}^{(r)}$ unter den Repräsentanten von $Q_{1}^{(r)}$ vorkommen, und es kommen dann unter den Repräsentanten von $Q_{1}$ Repräsentanten von $Q_{2}$ und $Q_{3}$ vor.

Weil aus $Q_{1}^{(m)} \equiv Q_{2}^{(n)}$ auch $Q_{1}^{(k)(m-k)} \equiv Q_{2}^{(k)(n-k)}$ folgt, wo $k<m<n$ ist, und also unter den Repräsentanten von $Q_{1}^{(k)}$ auch Repräsentanten von $Q_{2}^{(k)}$ vorkommen, so ergibt sich hieraus mit Hilfe des Satzes 20 in Nr. 34 und Nr. 35 :

Gehört $Q_{1}$ der ersten Klasse an, so gehören auch $Q_{2}, Q_{3}$ und sämtliche Repräsentanten von $Q_{1}, Q_{2}$ und $Q_{3}$ dieser Klasse an, und zwar derselben Art wie $Q_{1}$. Gehört ferner $Q_{1}$ der zweiten Klasse und erst $Q_{1}^{(m)}$ der ersten Klasse an, so gehören auch $Q_{2}, Q_{3}$ und alle die Repräsentanten von $Q_{1}, Q_{2}$ und $Q_{3}$, welche niedriger als die $m$-te sind, der zweiten Klasse an, und zwar derselben Art wie $Q_{1}$, und alle die Repräsentanten von $Q_{1}, Q_{2}$ und $Q_{3}$, welche höher als die $(m-1)$-te sind, der ersten Klasse an, und zwar der ersten oder der zweiten Art, je nachdem $Q_{1}$ in der zweiten Klasse der ersten oder der zweiten Art angehört.

45. Auch über die Art eines Elementes der ersten beiden Klassen gibt uns der Ordinatenwinkel $\frac{i}{k} \pi$ des Elementes Aufschluß, wo $\frac{i}{k}$ ein reduzierter echter Bruch ist.

Es gehört nämlich ein Element der ersten Klasse, für welches also der Nenner $k$ ungerade ist, nur dann der ersten Art an, wenn $k \equiv \pm 3^{2+v}\left(\bmod .3^{3+v}\right)$ und $\delta \equiv \pm 3^{v}\left(\bmod .3^{1+\nu}\right)$ ist, wo $v \geqq 0$ und $\delta$ der Exponent ist, zu dem die Zahl 2 in bezug auf den Modul $(k: 3)$ gehört; sonst gehört das 
Element der zweiten Art an. Ferner gehört ein Element der zweiten Klasse, für welches also $k$ gerade ist, wo etwa $k \equiv 2^{m}\left(\bmod .2^{m+1}\right)$, nur dann der ersten Art an, wenn $k \equiv \pm 3^{2+v}$ $\left(\bmod .3^{3+v}\right)$ und $\delta \equiv \pm 3^{v}\left(\bmod .3^{1+v}\right)$ ist, wo $v \geqq 0$ und $\delta$ der Exponent ist, zu dem die Zahl 2 in bezug auf den Modul $\left(k: 2^{m} .3\right)$ gehört; sonst gehört das Element der zweiten Art an.

Denn ein Element gehört nur dann der ersten Art der ersten Klasse an, wenn $\frac{i}{k}$ auf die Form $\frac{r}{3\left[2^{s}-(-1)^{s}\right]}$ gebracht werden kann, wo $r$ kein Vielfaches von 3 sein soll (Nr.36); es muß also dann, weil $i 3\left[2^{s}-(-1)^{s}\right]=r k$ ist (wo $i$ und $k$ relative Primzahlen sind und $r \not \equiv 0$ (mod. 3$)$ ist):

1)

und

2)

$$
2^{s} \equiv(-1)^{s}(\bmod . k: 3)
$$

sein, wenn

3)

und also

$$
k \equiv \pm 3^{v+2}\left(\bmod \cdot 3^{v+3}\right)
$$

ist, wo $v \geqq 0$ ist. Sind, umgekehrt, $k$ und $s$ solche Zahlen, die den Kongruenzen 1), 2), 3) und mithin auch 4) genügen, und ist $i$ eine zu $k$ relative Primzahl, so ist

$$
\frac{i}{k}=\frac{i \frac{3\left[2^{s}-(-1)^{s}\right]}{k},}{3\left[2^{s}-(-1)^{s}\right]},
$$

wo, weil $i 3\left[2^{s}-(-1)^{s}\right]$ ebenso wie $k$ nur durch $3^{v+2}$, nicht aber durch $3^{v+3}$ teilbar ist, $i \frac{3\left[2^{s}-(-1)^{s}\right]}{k}$ kein Vielfaches von 3 ist; mithin muß dann das Element, dessen Ordinatenwinkel $\frac{i}{k} \pi$ ist, der ersten Art der ersten Klasse angehören. Es gehört also ein Element nur dann der ersten Art der ersten Klasse an, wenn $k$ eine der Kongruenz 2) genügende Zahl ist und für diese Zahl $k$ eine den Kongruenzen 1) und 3) genügende Zahl $s$ existiert. Ist dies der Fall und ist dann etwa

$$
2^{n} \equiv(-1)^{n}(\bmod . k: 3)
$$

die Kongruenz niedrigsten Grades von der Gestalt 1), so ist einerseits nach 3 ), weil nach 1) $s$ ein Vielfaches von $n$ sein muß, 
$n$ kein Vielfaches von $3^{v+1}$, andererseits, weil nach 2) und 5) $2^{n}-(-1)^{n} \equiv(3-1)^{n}-(-1)^{n} \equiv 0\left(\bmod .3^{2+1}\right)$ sein $\operatorname{muß,} n \equiv 0$ $\left(\bmod .3^{v}\right)$, also muß

$$
n \equiv \pm 3^{v}\left(\bmod \cdot 3^{v+1}\right)
$$

sein; mithin muß dann, weil $n$ entweder $=\frac{\delta}{2}$, oder $=\delta$, oder $=2 \delta$ ist (nach Nr.42), auch

7)

$$
\delta \equiv \pm 3^{v}\left(\bmod .3^{v+1}\right)
$$

sein. Umgekehrt, folgt aus 7) auch 6) und 5); folglich gehört ein Element nur dann der ersten Art der ersten Klasse an, wenn $k$ und $\delta$ den Kongruenzen 2) und 7) genügen. Ferner gehört ein Element der zweiten Klasse, für welches $k \equiv 2^{m}\left(\bmod .2^{m+1}\right)$ ist, nur dann der ersten Art an, wenn der $m$-te Repräsentant des Elementes der ersten Art der ersten Klasse angehört; dieser $m$-te Repräsentant, dessen Ordinatenwinkel $\frac{i \pi}{k: 2^{m}}$ ist, gehört aber, wie soeben gesehen, nur dann der ersten Art der ersten Klasse an, wenn $k: 2^{m}$ und also auch $k$ der Kongruenz 2) und $\delta$ der Kongruenz 7) genügen, wo aber jetzt $\delta$ nicht mehr in bezug auf den Modul $\frac{k}{3}$, sondern in bezug auf den Modul $\frac{k}{2^{m} \cdot 3}$ genommen ist. 
Dritter Abschnitt.

\section{Über eine neue, spezielle Erzeugungsart der Kurven dritter Ordnung mit isoliertem Doppelpunkt bzw. dritter Klasse mit isolierter Doppeltangente.}

\section{\$ 8.}

46. Wir wollen nun das Gebilde untersuchen, welches von denjenigen Punkten gebildet wird, welche einem auf keiner Seite des Dreiecks $A B C$ liegenden reellen Punkte $P$ zugepaart sind in den von $A B C$ auf den sämtlichen durch $P$ gehenden Geraden $g$ erzeugten Punktinvolutionen $(g)^{2}$, und welches Gebilde mit $p^{3}$ bezeichnet werden mag.

Nennen wir zwei solche Punkte, die einander zugepaart sind in der von $A B C$ auf ihrer Verbindungsgeraden erzeugten Punktinvolution zweiten Grades, zwei konjugierte Punkte in bezug auf $A B C$, so besteht das Gebilde $p^{3}$ aus den sämtlichen zu $P$ in bezug auf $A B C$ konjugierten Punkten.

welches von denjenigen Strahlen gebildet wird, welche einer durch keine Ecke von $A B C$ gehenden reellen Geraden $p$ zugepaart sind in den von $A B C$ um die sämtlichen auf $p$ liegenden Punkte $Q$ erzeugten Strahleninvolutionen $(Q)^{2}$, und welches Gebilde mit $P^{3}$ bezeichnet werden mag.

Nennen wir zwei solche Gerade, die einander zugepaart sind in der von $A B C$ um ihren Schnittpunkt erzeugten Strahleninvolution zweiten Grades, zwei konjugierte Strahlen in bezug auf $A B C$, so besteht das Gebilde $P^{3}$ aus den sämtlichen zu $p$ in bezug auf $A B C$ konjugierten Strahlen.

Nun leuchtet sofort ein, daß das Gebilde $p^{3}$ die Ecken von $A B C$ und die drei Schnittpunkte $P_{a}, P_{b}$,

$P^{3}$ die Seiten von $A B C$ und die drei durch den Pol $P$ von 
$P_{c}$ der Polare $p$ von $P$ mit den Seiten von $A B C$ enthalten wird.

$p$ gehenden Ecktransversalen $p_{A}, p_{B}, p_{C}$ von $A B C$ enthalten wird.

Denn links ist jede Ecke allen Punkten, darunter auch $P$, der durch sie und $P$ gehenden Ecktransversalen zugepaart in der auf dieser von $A B C$ erzeugten parabolischen Punktinvolution (Nr.6). Ferner ist jeder der Schnittpunkte von $p$ mit den Dreieckseiten dem Pole $P$ von $p$ zugepaart in der von $A B C$ auf der ihn mit $P$ verbindenden Geraden erzeugten Punktinvolution zweiten Grades (Ende Nr.7).

47. Wir bedürfen nun, um einige Eigenschaften dieser Gebilde abzuleiten, des folgenden Hilfssatzes.

Hilfssatz 1 . Sind $k$ und $l$ irgend zwei Gerade, $K$ und $L$ ihre Pole, $v$ die $K$ mit $L$ verbindende Gerade und $V$ der Pol von $v$, so geht die Gerade, welche die beiden dem Schnittpunkte $k l$ in den auf $k$ und $l$ von $A B C$ erzeugten Punktinvolutionen $(k)^{2}$ und $(l)^{2}$ zugepaarten Punkte verbindet, durch $V$.

Und, umgekehrt, geht die Gerade, welche durch $V$ und durch den dem Schnittpunkte $k l$ in $(k)^{2}$ zugepaarten Punkt geht, auch durch den dem Schnittpunkte $k l$ in $(l)^{2}$ zupaarten Punkt.

Und dual.

Denn $k$ und $l$ sind Tangenten des Polarkegelschnitts $V^{2}$ von $v$; mithin müssen (Satz 3 in Nr. 10) $(k)^{2}$ und $(l)^{2}$ zu der von $A B C$ um $V$ erzeugten Strahleninvolution $(V)^{2}$ perspektiv sein, und es muß der Strahl, welcher in $(V)^{2}$ der Verbindungsgeraden von $V$ mit dem Schnittpunkte $k l$ zugepaart ist, durch die beiden dem $k l$ in $(k)^{2}$ und $(l)^{2}$ zugepaarten Punkte gehen, und es liegen also diese beiden Punkte mit $V$ in einer Geraden.

Die Umkehrung gilt nicht mehr, wenn $k$ durch eine Ecke von $A B C$ geht. Denn alsdann ist diese Ecke der Pol von $k$ und zugleich auch der Pol der Verbindungsgeraden $v$ der Pole von $k$ und irgendeiner andern Geraden $l$. Dieselbe Ecke ist aber dann in der parabolischen Involution $(k)^{2}$ allen Punkten von $k$, darunter auch kl, zugepaart; mithin bleibt dann die Gerade, welche den Pol von $v$ mit dem dem $k l$ in $(k)^{2}$ zugepaarten Punkte verbindet, unbestimmt. 
48. Nun können wir allererst nachweisen, daß das Gebilde $p^{3}$ mit keiner Geraden mehr als drei Punkte gemein haben kann.

Bew eis. Sollte irgendeine Gerade $d$ mit $p^{3}$ vier Punkte gemein haben und also vier durch $P$ gehende Geraden $g_{1}, g_{2}, g_{3}$ und $g_{4}$, wo notwendig mindestens eine dieser vier Geraden, etwa $g_{1}$, keine der drei durch $P$ gehenden Ecktransversalen ist, in denjenigen Punkten $P_{1}, P_{2}, P_{3}$ und $\left.P_{4}{ }^{1}\right)$ schneiden, welche dem $P$ zugepaart sind in den Punktinvolutionen $\left(g_{1}\right)^{2},\left(g_{2}\right)^{2},\left(g_{3}\right)^{2}$ und $\left(g_{4}\right)^{2}$, so würde nach Hilfssatz 1 die Gerade $d$, welche in $\left(g_{1}\right)^{2}$ und $\left(g_{2}\right)^{2}$, in $\left(g_{1}\right)^{2}$ und $\left(g_{3}\right)^{2}$ und in $\left(g_{1}\right)^{2}$ und $\left(g_{4}\right)^{2}$ je die beiden dem gemeinsamen Punkte $P$ ihrer Träger zugepaarten Punkte verbindet, durch die Pole derjenigen drei Geraden gehen müssen, welche den Pol $G_{1}$ von $g_{1}$ mit den Polen $G_{2}, G_{3}, G_{4}$ von $g_{2}, g_{3}, g_{4}$ verbinden, was aber unmöglich ist; da die Pole der durch $G_{1}$ gehenden Geraden (wo $G_{1}$, als Pol einer von den Ecktransversalen und Seiten von $A B C$ verschiedenen Geraden $g_{1}$, auf keiner Seite von $A B C$ liegt) einen Kegelschnitt bilden (Nr.2) und keine drei dieser Pole also in einer Geraden liegen können.

49. Ferner können wir nachweisen, daß, wenn das Gebilde $p^{3}$ mit einer Geraden zwei reelle Punkte gemein hat, es mit derselben Geraden noch einen dritten reellen gemein haben muß.

Beweis. Sind die zwei gemeinschaftlichen Punkte von $p^{3}$ mit der Geraden zwei der Ecken von $A B C$ und ist die Gerade also eine der Seiten von $A B C$, so hat diese Seite mit $p^{3}$ noch einen dritten reellen Punkt gemein, nämlich ihren Schnittpunkt mit der Polare $p$ von $P$ (Nr.46). Von diesem Falle können wir nun absehen und nehmen an, daß von den beiden $p^{3}$ und der Geraden, welche letztere $r$ heiße, gemeinsamen reellen Punkten, welche $P_{i}$ und $P_{k}$ heißen mögen und dem $P$ in $\left(g_{i}\right)^{2}$ und $\left(g_{k}\right)^{2}$ zugepaart sind, mindestens einer, etwa $P_{i}$, keine der Ecken von $A B C$ ist. Die Gerade $r$ wird dann nach Hilfssatz 1 durch den reellen Pol der reellen Verbindungsgeraden $G_{i} G_{k}$ der Pole von $g_{i}$ und $g_{k}$ gehen und mithin, weil der Pol von $G_{i} G_{k}$ auf dem Polarkegelschnitt $g_{i}^{2}$ des auf keiner Seite von $A B C$ liegenden Punktes $G_{i}$

1) Von nun an soll, wenn irgendein Punkt von $p^{3}$ mit $P_{i}$ bezeichnet wird, die diesen Punkt von $P$ aus projizierende Gerade mit $g_{i}$, ihr Pol mit $G_{i}$ und ihr Schnittpunkt mit der Polare $p$ von $P$ mit $Q_{i}$, also stets mit einem und demselben Index bezeichnet werden. 
liegt, $g_{i}^{2}$ noch in einem zweiten reellen Punkte treffen. Dieser zweite Schnittpunkt von $r$ mit $g_{i}^{2}$ ist aber $\mathrm{Pol}^{1}$ ) einer durch $G_{i}$ gehenden Geraden, welche letzte, weil $G_{i}$, der Pol der durch $P$ gehenden Geraden $g_{i}$, auf dem Polarkegelschnitt ${ }^{2}$ ) $p^{2}$ von $P$ liegt, noch durch einen zweiten reellen Punkt, etwa $G_{l}$, von $p^{2}$ gehen muß. Nun ist aber der auf $p^{2}$ liegende reelle Punkt $G_{l}$ Pol der durch $P$ gehenden reellen Geraden $g_{l}$. Folglich muß nach der Umkehrung des Hilfssatzes 1 die Gerade $r$, welche durch den Pol der Verbindungsgeraden $G_{i} G_{l}$ der Pole von $g_{i}$ und $g_{l}$ und durch den dem gemeinsamen Punkt $P$ von $g_{i}$ und $g_{l}$ in $\left(g_{i}\right)^{2}$ zugepaarten Punkt $P_{i}$ geht, auch durch den dem $P$ in $\left(g_{l}\right)^{2}$ zugepaarten Punkt $P_{l}$, welcher $p^{3}$ angehört, gehen und also mit $p^{3}$ noch einen dritten reellen Punkt gemein haben.

50. Zugleich haben wir Folgendes erkannt:

I. Eine durch einen $p^{3}$ angehörigen, von den Ecken $A$, $B, C$ aber verschiedenen Punkt $P_{i}$ gehende Gerade $r$, welche mit $p^{3}$ noch die beiden von $P$ aus durch $g_{k}$ und $g_{l}$ projizierten Punkte $P_{k}$ und $P_{l}$ gemein hat, hat mit dem Polarkegelschnitt $g_{i}^{2}$ von $G_{i}$, dem Pole $\operatorname{der} P_{i}$ von $P$ aus projizierenden Geraden $g_{i}$, diejenigen beiden Punkte $V_{i k}, V_{i l}$ und nur diejenigen beiden gemein, welche Pole der beiden Verbindungsgeraden $v_{i k}, v_{i l}$ von $G_{i}$ mit bzw. den Polen $G_{k}$ und $G_{l}$ von $g_{k}$ und $g_{l}$ sind.

Umgekehrt hat eine durch einen solchen Punkt $\boldsymbol{P}_{i}$ gehende Gerade $r$, welche mit $g_{i}^{2}$ die beiden Punkte $V_{i k}$ und $V_{i l}$ gemein hat, mit $p^{3}$ außer $P_{i}$ noch diejenigen beiden Punkte $P_{k}, P_{l}$ und nur diejenigen beiden gemein, welche von $P$ aus durch diejenigen Geraden $g_{k}$ und $g_{l}$ projiziert werden, deren Pole $G_{k}$ und $G_{l}$ mit $G_{i}$, dem Pole der $P_{i}$ von $P$ aus projizierenden Geraden $g_{i}$, verbunden die Polaren $v_{i k}$ und $v_{i l}$ von $V_{i k}$ und $V_{i l}$ liefern.

Denn die Gerade, die in $\left(g_{i}\right)^{2}$ und $\left(g_{k}\right)^{2}$, in $\left(g_{i}\right)^{2}$ und $\left(g_{l}\right)^{2}$ die beiden dem $g_{i}, g_{k}, g_{l}$ gemeinsamen Punkte $P$ zugepaarten Punkte $P_{i}$ und $P_{k}, P_{i}$ und $P_{l}$ verbindet, muß nach Hilfssatz 1 durch die auf $g_{i}^{2}$ liegenden Pole $V_{i k}$ und $V_{l l}$ von $v_{i k} \equiv G_{i} G_{k}$ und $v_{i l} \equiv G_{i} G_{l}$ gehen;

1) In bezug auf $A B C$; s. oben Fußnote auf S. 12.

2) Hier und überall im folgenden, wo von Polarkegelschnitten schlechthin die Rede ist, sollen diese in bezug auf $A B C$ verstanden werden. 
und die Gerade, die durch die Pole $V_{i k}$ und $V_{i l}$ von $G_{i} G_{k}$ und $G_{i} G_{l}$ und durch den dem $P \equiv g_{i} g_{k} \equiv g_{i} g_{l}$ in $\left(g_{i}\right)^{2}$ zugepaarten Punkt $P_{i}$ geht, muß nach der Umkehrung des Hilfssatzes 1 auch durch die dem $P$ in $\left(g_{k}\right)^{2}$ und in $\left(g_{l}\right)^{2}$ zugepaarten Punkte $P_{k}$ und $P_{l}$ gehen; und keine Gerade kann mit $g_{i}^{2}$ und $p^{3}$ mehr als zwei bzw. mehr als drei Punkte gemein haben.

Hieraus ergibt sich: Die durch $P_{i}$ gehende Gerade $r$ ist dann und nur dann Tangente an $p^{3}$ in $P_{k}$, wenn sie Tangente an $g_{i}^{2}$ ist. Denn dann und nur dann sind die beiden $g_{i}^{2}$ und $r$ gemeinsamen Punkte $V_{i k}$ und $V_{i l}$ und mithin auch ihre Polaren $v_{i k} \equiv G_{i} G_{k}$ und $v_{i l} \equiv G_{i} G_{l}$ und also auch $G_{k}$ und $G_{l}, g_{k}$ und $g_{l}$ und endlich auch die beiden Punkte $P_{k}$ und $P_{l}$ je einander unendlich nahe. Ferner ist dann und nur dann die durch $P_{i}$ gehende Gerade $r$ Tangente an $p^{3}$ in demselben Punkte $P_{i}$, wenn sie durch den auf $p_{i}^{2}$ liegenden Pol $T_{i}$ der Tangente $t_{i}$ an $p^{2}$ im Punkte $G_{i}$ geht. Denn dann und nur dann, wenn einer der beiden $r$ und $g_{i}^{2}$ gemeinsamen Punkte, etwa $V_{i k}$, mit $T_{i}$ und also $v_{i k} \equiv G_{i} G_{k}$ mit der Tangente $t_{i}$ an $p^{2}$ in $G_{i}$ identisch ist, sind $G_{i}$ und $G_{k}$ und mithin auch $g_{i}$ und $g_{k}$ und also auch $P_{i}$ und $P_{k}$ je einander unendlich nahe. Wendetangente an $p^{3}$ in $P_{i}$ ist aber die Gerade $r$ dann und nur dann, wenn sie Tangente an $g_{i}^{2}$ in $T_{i}$ ist. Denn dann und nur dann liegen die beiden $r$ und $g_{i}^{2}$ gemeinsamen Punkte $V_{i k}$ und $V_{i l}$ im Berührungspunkte $T_{i}$ und mithin auch $v_{i k} \equiv G_{i} G_{k}$ und $v_{i l} \equiv G_{i} G_{l}$ in der Tangente $t_{i}$ an $p^{2}$ in $G_{i}$, und es sind also nur dann alle drei Punkte $G_{i}, G_{k}, G_{l}$ und alle drei Polaren $g_{i}, g_{k}, g_{l}$ und folglich auch alle drei Punkte $P_{i}$, $P_{k}, P_{l}$ je einander unendlich nahe. Endlich hat die Gerade $r$ dann und nur dann drei voneinander verschiedene Punkte $P_{i}$, $P_{k}, P_{l}$ mit $p^{3}$ gemein, wenn sie von $g_{i}^{2}$ in zwei voneinander und von $T_{i}$ verschiedenen Punkten getroffen wird. Denn dann und nur dann sind $T_{i}, V_{i k}, V_{i l}$ und mithin auch $t_{i}, v_{i k} \equiv G_{i} G_{k}$, $v_{i l} \equiv G_{i} G_{l}$ und $G_{i}, G_{k}, G_{l}$ und $g_{i}, g_{k}, g_{l}$ und $P_{i}, P_{k}, P_{l}$ sämtlich voneinander verschieden. Ist $P_{i}$ reell, so sind hiernach auch $P_{k}$ und $P_{l}$ dann und nur dann reell, wenn $r$ von $g_{i}^{2}$ in zwei reellen Punkten getroffen wird.

51. Hiermit sind die folgenden Aufgaben gelöst.

Aufgabe 1. Auf einer durch einen Punkt $P_{i}$ von $p^{3}$ gehenden Geraden $r$ deren beide weitere Schnittpunkte mit 
$p^{3}$ zu ermitteln, wobei $P_{i}$ von $P$ aus durch die Gerade $g_{i}$ projiziert wird.

Auflösung. Man ermittelt zuerst die beiden Schnittpunkte $V_{i k}$ und $V_{i l}$ von $r$ mit dem Polarkegelschnitt (in bezug auf $A B C$ ) $g_{i}^{2}$ von $G_{i}$, dem auf dem Polarkegelschnitt $p^{2}$ von $P$ liegenden Pole von $g_{i}$, und ihre durch $G_{i}$ gehenden Polaren $v_{i k}$ und $v_{i l}$, sodann die beiden zweiten Schnittpunkte $G_{k}$ und $G_{l}$ von $v_{i k}$ und $v_{i l}$ mit $p^{2}$ und ihre durch $P$ gehenden Polaren $g_{k}$ und $g_{l}$; alsdann sind die beiden Schnittpunkte $P_{k}$ und $P_{l}$ von $r$ mit $g_{k}$ und $g_{l}$ die gesuchten Schnittpunkte von $r$ mit $p^{3}$.

Diese Lösung ist aber, nach dem Vorhergehenden, nur dann anwendbar, wenn $P_{i}$ keine der Ecken von $A B C$ ist.

Anmerkung. Diese Lösung liefert auch dann die beiden weiteren Schnittpunkte $P_{k}$ und $P_{l}$ von $r$ mit $p^{3}$, wenn $r$ ganz außerhalb $g_{i}^{2}$ liegt, nur sind dann $P_{k}$ und $P_{l}$ ebenso wie die beiden Schnittpunkte von $r$ mit $g_{i}^{2}$ konjugiert-imaginär, wenn nur $P_{i}$ und $r$ reell sind. Denn, wie man sich überzeugen kann, gelten alle Resultate des ersten Abschnittes, darunter der Satz 3 in Nr. 10, und mithin der Hilfssatz 1 in Nr. 47 und die Aussage I auch für imaginäre Punkte und Gerade. Nun müssen aber $v_{i k}$ und $v_{i l}$ zugleich mit ihren Polen $V_{i k}$ und $V_{i l}$, den beiden Schnittpunkten von $r$ mit $g_{i}^{2}$, konjugiert-imaginär sein (nach Nr. 3), und alsdann müssen auch die beiden zweiten Schnittpunkte $G_{k}$ und $G_{l}$ des reellen Kegelschnitts $p^{2}$ mit den konjugiert-imaginären Geraden $v_{i k}$ und $v_{i l}$, deren reeller Schnittpunkt $G_{i}$ auf dem nämlichen Kegelschnitt $p^{2}$ liegt, konjugiert-imaginär sein (es wird nämlich die reelle, elliptische Strahleninvolution um $G_{i}$, deren Doppelstrahlen $v_{i k}$ und $v_{i l}$ sind, von $p^{2}$ in einer reellen, elliptischen, krummen Punktinvolution geschnitten, deren Doppelpunkte $G_{k}$ und $G_{l}$ sind) und mithin auch ihre Polaren $g_{k}$ und $g_{l}$; folglich müssen auch $P_{k}$ und $P_{l}$, die nunmehr Schnittpunkte der reellen Geraden $r$ mit den konjugiert-imaginären Geraden $g_{k}$ und $g_{l}$ sind, konjugiert-imaginär sein. Auch wenn $P_{i}$ oder $r$ oder beide zugleich imaginär sind, liefert die gegebene Lösung die beiden weitern Schnittpunkte von $r$ mit $p^{3}$. Eine durch $P_{i}$ gehende Gerade hat also immer mit $p^{3}$ noch zwei Punkte gemein, welche aber auch imaginär sein können.

Berliner, Habilitationsschrift. 
Aufgabe 2. Die von dem Punkte $P_{i}$ an $p^{3}$ gehenden Tangenten (außer der Tangente in $P_{i}$ selbst) zu ermitteln.

Auflösung. Man ermittelt die beiden von $P_{i}$ an $g_{i}^{2}$ gehenden Tangenten, diese und nur diese sind zugleich die gesuchten Tangenten an $p^{3}$.

Weil $P_{i}$, wenn er reell ist, auf der immer ganz außerhalb des Polarkegelschnitts $g_{i}^{2}$ verlaufenden Geraden $g_{i}$ (nach Nr. 2) liegt, so folgt hieraus, daß von jedem reellen Punkte von $p^{3}$ (die Ecken von $A B C$ vorläufig ausgenommen) außer der Tangente in dem Punkte selbst noch zwei reelle Tangenten an $p^{3}$ gehen.

Aufgabe 3. Die Tangente im Punkte $P_{i}$ von $p^{3} \mathrm{zu}$ ermitteln.

Auflösung. Man ermittelt den Pol $G_{i}$ von $g_{i}$, welcher Pol auf dem Polarkegelschnitt $p^{2}$ von $P$ liegt, sodann die Tangente $t_{i}$ an $p^{2}$ in $G_{i}$ und den Pol $T_{i}$ von $t_{i}$; die Verbindungsgerade von $P_{i}$ mit $T_{i}$ wird dann die gesuchte Tangente von $p^{3}$ sein.

Auch die Lösungen der Aufgaben 2 und 3 sind nur dann anzuwenden, wenn $P_{i}$ keine der Ecken von $A B C$ ist.

Die entsprechenden Aufgaben für das duale Gebilde $P^{3}$ sind dual zu lösen.

Zugleich haben wir das folgende Kriterium gewonnen.

Satz 23. Ist $P_{i}$ ein von den Ecken von $A B C$ verschiedener, reeller Punkt von $p^{3}, g_{i}$ die $P_{i}$ von $P$ aus projizierende Gerade, $G_{i}$ deren Pol, $g_{i}^{2}$ der Polarkegelschnitt von $G_{i}, p^{2}$ der Polarkegelschnitt von $P$, welcher letztere Polarkegelschnitt durch den $\mathrm{Pol} G_{i}$ von $g_{i}$ geht, $t_{i}$ die Tangente an $p^{2}$ in $G_{i}, T_{i}$ der Pol von $t_{i}$, welcher Pol auf $g_{i}^{2}$ liegt, und $r$ irgendeine durch $P_{i}$ gehende, reelle Gerade, so sind die beiden weitern
Ist $p_{i}$ ein von den Seiten von $A B C$ verschiedener, reeller Strahl von $P^{3}, Q_{i}$ der Punkt, in dem $p$ von $p_{i}$ geschnitten wird, $q_{i}$ dessen Polare, $Q_{i}^{2}$ der Polarkegelschnitt von $q_{i}, P^{2}$ der Polarkegelschnitt von $p$, welcher letztere Polarkegelschnitt von der Polare $q_{i}$ von $Q_{i}$ tangiert wird, $U_{i}$ der Berührungspunkt von $q_{i}$ mit $P^{2}, u_{i}$ die Polare von $U_{i}$, welche Polare $Q_{i}^{2}$ tangiert, und $N$ irgendein auf $p_{i}$ liegender, reeller Punkt, 
Schnittpunkte $P_{k}$ und $P_{l}$ von $\boldsymbol{r}$ mit $p^{3}$ konjugiert-imaginär, oder reell und voneinander und von $P_{i}$ verschieden, oder zwar voneinander, aber $P_{k}$ ist nicht von $P_{i}$ verschieden und $r$ ist also die Tangente an $p^{3}$ in $P_{i}$, oder $P_{k}$ und $P_{l}$ sind zwar von $P_{i}$, aber nicht voneinander verschieden und $r$ ist also die Tangente an $p^{3}$ in $P_{k}$, oder endlich ist weder $P_{k}$ noch $P_{l}$ von $P_{i}$ verschieden und $r$ ist also eine Wendetangente von $p^{3}$ in $P_{i}$, je nachdem $r$ mit $g_{i}^{2}$ zwei konjugiert-imaginäre, oder zwei reelle, voneinander und von $T_{i}$ verschiedene Punkte, oder $T_{i}$ und noch einen andern Punkt gemein hat, oder $r$ Tangente an $g_{i}^{2}$ in einem von $T_{i}$ verschiedenen Punkte ist, oder endlich $r$ Tangente an $g_{i}^{2}$ in $T_{i}$ ist; und umgekehrt. so sind die beiden weitern durch $N$ gehenden Strahlen $p_{m}$ und $p_{n}$ von $P^{3}$ konjugiert-imaginär, oder reell und voneinander und von $p_{i}$ verschieden, oder zwar voneinander, aber $p_{m}$ ist nicht von $p_{i}$ verschieden und $N$ ist also der Berührungspunkt von $p_{i}$ mit $P^{3}$, oder $p_{m}$ und $p_{n}$ sind zwar von $p_{i}$, aber nicht voneinander verschieden und $N$ ist also der Berührungspunkt von $p_{m}$ mit $P^{3}$, oder endlich ist weder $p_{m}$ noch $p_{n}$ von $p_{i}$ verschieden und $N$ ist also ein Rückkehrpunkt von $P^{3}$ auf $p_{i}$, je nachdem von $N$ zwei konjugiert-imaginäre, oder zwei reelle voneinander und von $u_{i}$ verschiedene Tangenten, oder $u_{i}$ und noch eine andere Tangente an $Q_{i}^{2}$ gehen, oder $N$ Berührungspunkt in einer von $u_{i}$ verschiedenen Tangente von $Q_{i}^{2}$ ist, oder endlich $N$ Berührungspunkt in der Tangente $u_{i}$ von $Q_{i}^{2}$ ist; und umgekehrt.

\section{§ 9.}

52. Das im letzten Satze gegebene Kriterium der Realität und der Lage der beiden weitern Schnittpunkte von $p^{3}$ mit einer durch einen reellen Punkt $P_{i}$ von $p^{3}$ gehenden Geraden versagt aber, wenn $P_{i}$ eine der Ecken von $A B C$ ist; da alsdann der Hilfssatz 1 (und nämlich seine Umkehrung), auf dem das Kriterium beruht, nicht mehr zur Anwendung gebracht werden kann (Nr. 47), 
auch ist dann dieselbe Ecke zugleich der Pol $G_{i}$ von $g_{i}$ und der Polarkegelschnitt $g_{i}^{2}$ reduziert sich dann auf die beiden durch die nämliche Ecke gehenden Seiten von $A B C$ (Nr. 2).

Wir wollen deshalb aus diesem Kriterium ein zweites ableiten, welches zweite seine Gültigkeit auch für die Ecken von $A B C$ beibehalten wird.

\section{$\mathrm{Zu}$ diesem Ende beweisen wir den folgenden}

Hilfssatz 2. Die Tangente $t$ in irgendeinem Punkte $G$ des Polarkegelschnitts $p^{2}$ von $P$ ist zu der ihren Berührungspunkt $G$ mit $P$ verbindenden Geraden zugepaart in der von $A B C$ um $G$ erzeugten Strahleninvolution $(G)^{2}$; und dual.

Beweis. Weil $p$ die Polare von $P$ auch in bezug auf $p^{2}$ ist (Nr. 2), so muß der $\mathrm{Pol}$ der Verbindungsgeraden $G P$ in bezug auf $p^{2}$ der Schnittpunkt der Tangente $t$ mit $p$ sein. Es sind also die beiden Punkte, in denen $p$ von $t$ und GP geschnitten wird, einander zugepaart in der Involution der in bezug auf $p^{2}$ konjugierten Punkte auf $p$ und also auch in der mit dieser identischen (Nr. 2) von $A B C$ erzeugten Involution $(p)^{2}$. Nach Satz 3 (Nr. 10) ist aber $(p)^{2}$ zu der von $A B C$ um den auf $p^{2}$ liegenden Punkt $G$ erzeugten Strahleninvolution $(G)^{2}$ perspektiv; folglich müssen die beiden Geraden $t$ und $G P$, welche von $G$ aus ein Punktepaar in $(p)^{2}$ projizieren, ein Strahlenpaar in $(G)^{2}$ bilden. (Diesen Satz habe ich in meiner Dissertation Nr. 24 auf andere Weise bewiesen.)

53. Ist nun $G_{i}$ irgendein auf $p^{2}$ liegender Punkt, $g_{i}$ seine durch $P$ gehende Polare und sind $G_{i 1}$ und $G_{i 2}$ die beiden Schnittpunkte von $p^{2}$ mit $g_{i}{ }^{1}$ ) und $g_{i 1}$ und $g_{i 2}$ ihre durch $P$ gehenden Polaren, so muß nach Satz 3 (Nr. 10) die von $A B C$ erzeugte Strahleninvolution $\left(G_{i}\right)^{2}$ zu den von $A B C$ erzeugten Punktinvolutionen $\left(g_{i}\right)^{2},\left(g_{i 1}\right)^{2},\left(g_{i 2}\right)^{2}$ perspektiv sein. Folglich muß die Tangente $t_{i}$ an $p^{2}$ im Punkte $G_{i}$, welche dem Strahle $G_{i} P$ in $\left(G_{i}\right)^{2}$ zugepaart ist (Hilfssatz 2), durch diejenigen drei Punkte $P_{i}, P_{i 1}, P_{i 2}$ gehen, welche dem $P$ in $\left(g_{i}\right)^{2},\left(g_{i 1}\right)^{2},\left(g_{i 2}\right)^{2}$ zugepaart sind und welche also $p^{3}$ angehören. Hieraus folgt weiter nach Hilfssatz 1 , daß die Tangente $t_{i}$, die nunmehr die beiden dem

1) Ist $G_{i}$ und mithin auch $g_{i}$ reell, so sind auch $G_{i 1}$ und $G_{i 2}$ reell, da $g_{i}$ durch den innerhalb $p^{2}$ liegenden Punkt $P$ (Nr. 2) geht. 
$P \equiv g_{i} g_{i 1} \equiv g_{i} g_{i 2}$ in $\left(g_{i}\right)^{2}$ und $\left(g_{i 1}\right)^{2}$, in $\left(g_{i}\right)^{2}$ und $\left(g_{i 2}\right)^{2}$ zugepaarten Punkte $P_{i}$ und $P_{i 1}, P_{i}$ und $P_{i 2}$ verbindet, auch durch die Pole der beiden $G_{i}$ mit $G_{i 1}$ und $G_{i 2}$ verbindenden Geraden gehen muß.

Bemerken wir noch, daß die beiden durch $P$ gehenden Geraden $g_{i 1}$ und $g_{i 2}$ und ebenso die beiden Verbindungsgeraden $G_{i} G_{i 1}$ und $G_{i} G_{i 2}$, deren Pole mit $P$ bzw. mit $G_{i}$ in je einer Geraden liegen, nämlich in $g_{i}$ bezw. in $t_{i}$, ein Strahlenpaar in $(P)^{2}$ bzw. in $\left(G_{i}\right)^{2}$ bilden (Satz 1 in Nr. 7), und daß das Strahlenpaar $g_{i 1}$ und $g_{i 2}$ durch $g_{i}$, den ersten Repräsentanten von $g_{i 1}$ und $g_{i 2}$, und $P G_{i}$, den zweiten Repräsentanten von $g_{i 1}$ und $g_{i 2}$, um $P$ harmonisch getrennt wird (Satz 17 in Nr. 28), so ergibt sich:

Satz 24. Auf der Tangente $t_{i}$ in einem Punkte $G_{i}$ des Polarkegelschnitts $p^{2}$ von $P$ liegen die drei $p^{3}$ angehörenden Punkte, welche von $P$ aus durch die Polare $g_{i}$ von $G_{i}$ und durch dasjenige Strahlenpaar in $(P)^{2}$ projiziert werden, dessen Pole auf $g_{i}$ liegen und also die Schnittpunkte von $g_{i}$ mit $p^{2}$ sind; und zwar ist auf $t_{i}$ der Berührungspunkt $G_{i}$ von dem durch $g_{i}$ projizierten Punkte von $p^{3}$ durch die beiden andern Punkte von $p^{3}$ harmonisch getrennt. Ferner liegen auf derselben Tangente $t_{i}$ die beiden Pole desjenigen Strahlenpaares in $\left(G_{i}\right)^{2}$, welches $G_{i}$ mit jenen beiden Schnittpunkten von $g_{i}$ mit $p^{2}$ verbindet.
Durch den Berührungspunkt $U_{i}$ einer Tangente $q_{i}$ des Polarkegelschnitts $P^{2}$ von $p$ gehen die drei $P^{3}$ angehörenden Strahlen, welche von $p$ in dem Pole $Q_{i}$ von $q_{i}$ und in demjenigen Punktepaare in $(p)^{2}$ geschnitten werden, dessen Polaren durch $Q_{i}$ gehen und also die beiden von $Q_{i}$ an $P^{2}$ gehenden Tangenten sind; und zwar ist um $U_{i}$ die Tangente $q_{i}$ von dem durch $Q_{i}$ gehenden Strahle von $P^{3}$ durch die beiden andern Strahlen von $P^{3}$ harmonisch getrennt. Ferner gehen durch denselben Berührungspunkt $U_{i}$ die beiden Polaren desjenigen Punktepaares in $\left(q_{i}\right)^{2}$, in welchem $q_{i}$ von jenen beiden von $Q_{i}$ an $P^{2}$ gehenden Tangenten geschnitten wird.

54. Ist nun wieder $P_{i}$ irgendein von den Ecken von $A B C$ verschiedener Punkt von $p^{3}, G_{i}$ der auf dem Polarkegelschnitt $p^{2}$ 
von $P$ liegende $\mathrm{Pol}$ der $P_{i}$ von $P$ aus projizierenden Geraden $g_{i}$, $g_{i}^{2}$ der Polarkegelschnitt von $G_{i}$ und sind $t_{i^{\prime}}$ und $t_{i^{\prime \prime}}$ die beiden von $P_{i}$ an $p^{3}$ gehenden Tangenten (außer der Tangente an $p^{3}$ in $P_{i}$ selbst), welche zugleich die von $P_{i}$ an $g_{i}^{2}$ gehenden Tangenten sind und welche stets reell sind, wenn nur $P_{i}$ reell ist (Nr. 51), $P_{i^{\prime}}$ und $P_{i^{\prime \prime}}, V_{i^{\prime}}$ und $V_{i^{\prime \prime}}$ ihre Berührungspunkte mit $p^{3}$ bezw. mit $g_{i}^{2}, g_{i^{\prime}}$ und $g_{i^{\prime \prime}}$ die $P_{i^{\prime}}$ und $P_{i^{\prime \prime}}$ von $P$ aus projizierenden Geraden, $G_{i^{\prime}}$ und $G_{i^{\prime \prime}}$ die auf $p^{2}$ liegenden Pole von $g_{i^{\prime}}$ und $g_{i^{\prime \prime}}$ und endlich $v_{i^{\prime}}$ und $v_{i^{\prime \prime}}$ die durch $G_{i}$ gehenden Polaren von $V_{i^{\prime}}$ und $V_{i^{\prime}}$, so müssen nach Nr. $50 G_{i^{\prime}}$ und $G_{i^{\prime \prime}}$ die je zweiten Schnittpunkte von $v_{i^{\prime}}$ und $v_{i^{\prime \prime}}$ mit $p^{2}$ sein. Nun müssen aber die Berührungspunkte $V_{i^{\prime}}$ und $V_{i^{\prime \prime}}$ der beiden von $P_{i}$ an $g_{i}^{2}$ gehenden Tangenten auf der Polare von $P_{i}$ in bezug auf $g_{i}^{2}$ liegen, welche Polare die Verbindungsgerade $G_{i} P$ ist; da in be $\mathrm{z}_{\mathrm{ug}}$ auf $g_{i}^{2} P_{i}$, der dem $P$ in $\left(g_{i}\right)^{2}$ zugepaarte Punkt, dem $P$ konjugiert ist, und $G_{i}$ der Pol von $g_{i}$ (nach Nr. 2). Wenn also $G_{i 1}$ und $G_{i 2}$ die beiden Schnittpunkte von $g_{i}$ mit $p^{2}$ sind, so müssen $v_{i^{\prime}} \equiv G_{i} G_{i^{\prime}}$ und $v_{i^{\prime \prime}} \equiv G_{i} G_{i^{\prime \prime}}$, deren Pole $V_{i^{\prime}}$ und $V_{i^{\prime \prime}}$ von $G_{i}$ aus durch die nämliche Gerade $G_{i} P$ projiziert werden, und die beiden Verbindungsgeraden $G_{i} G_{i 1}$ und $G_{i} G_{i 2}$, deren Pole von $G_{i}$ aus durch die Tangente $t_{i}$ von $p^{2}$ in $G_{i}$ projiziert werden (Satz 24), nach der Anmerkung in Nr. 22, weil $G_{i} P$ und $t_{i}$ ein Strahlenpaar in $\left(G_{i}\right)^{2}$ bilden (Hilfssatz 2), zwei durcheinander harmonisch getrennte Strahlenpaare in $\left(G_{i}\right)^{2}$ bilden. Weil aber die auf $p^{2}$ induzierte Punktinvolution $\left(p^{2}\right)^{2}$ von dem auf $p^{2}$ liegenden Punkte $G_{i}$ durch die Strahleninvolution $\left(G_{i}\right)^{2}$ projiziert wird (Nr. 10), so müssen die zweiten Schnittpunkte $G_{i^{\prime}} G_{i^{\prime \prime}}$ und $G_{i 1} G_{i 2}$ von $p^{2}$ mit jenen beiden durcheinander harmonisch getrennten Strahlenpaaren $G_{i}\left(G_{i^{\prime}} G_{i^{\prime \prime}}, G_{i 1} G_{i 2}\right)$ von $\left(G_{i}\right)^{2}$ zwei durcheinander harmonisch getrennte Punktepaare in $\left(p^{2}\right)^{2}$ bilden. Weil ferner vier harmonische Punkte auf einem Kegelschnitt durch zwei in bezug auf diesen konjugierte Gerade eingeschnitten werden und die Involution der in bezug auf $p^{2}$ konjugierten Strahlen um $P$ mit $(P)^{2}$ identisch ist (Nr. 2), so müssen nun die beiden durcheinander harmonisch getrennten Punktepaare $G_{i^{\prime}} G_{i^{\prime \prime}}$ und $G_{i 1} G_{i 2}$ der induzierten Involution $\left(p^{2}\right)^{2}$, deren Zentrum $P$ ist (Nr. 7), von $P$ aus durch ein Strahlenpaar in $(P)^{2}$ projiziert werden. Nunmehr sind aber $G_{i 1}$ und $G_{i 2}$ die Schnittpunkte von $p^{2}$ mit der durch $P$ gehenden Geraden $g_{i}$; mithin ergibt sich: 
I. Die Pole $G_{i^{\prime}}$ und $G_{i^{\prime \prime}}$ derjenigen beiden Geraden $g_{i^{\prime}}$ und $g_{i^{\prime \prime}}$, welche die Berührungspunkte $P_{i^{\prime}}$ und $P_{i^{\prime \prime}}$ der beiden von einem $p^{3}$ angehörenden Punkte $P_{i}$ an $p^{3}$ gehenden Tagenten (außer der Tangente in $P_{i}$ selbst) von $P$ aus projizieren und welche, wie wir sahen, ein Strahlenpaar in $(P)^{2}$ bilden, liegen auf der zu $g_{i}$ in $(P)^{2}$ zugepaarten Geraden.

55. Sind nun $V_{i k}$ und $V_{i l}$ die beiden Schnittpunkte von $g_{i}^{2}$ mit irgendeiner durch $P_{i}$ gehenden Geraden $r, v_{i k}$ und $v_{i l}$ ihre durch den auf $p^{2}$ liegenden Punkt $G_{i}$ gehenden Polaren und $G_{k}$ und $G_{l}$ die zweiten Schnittpunkte von $p^{2}$ mit $v_{i k}$ und $v_{i l}$, so müssen $V_{i k} V_{i l} V_{i^{\prime}} V_{i^{\prime \prime}}$ vier harmonische Punkte auf $g_{i}^{2}$ sein, da die Berührungspunkte $V_{i^{\prime}}$ und $V_{i^{\prime \prime}}$ der beiden von $P_{i}$ an $g_{i}^{2}$ gehenden Tangenten auf einer zu $r \equiv V_{i k} V_{i l}$ in bezug auf $g_{i}^{2}$ konjugierten Geraden, nämlich auf der Polare von $P_{i}$ in bezug auf $g_{i}^{2}$, liegen. Weil aber der Büschel der Polaren um $G_{i}$ zu der Punktreihe der Pole auf $g_{i}^{2}$ projektiv ist (nach Nr.2), so müssen nun $v_{i k} v_{i l} v_{i^{\prime}} v_{i^{\prime \prime}} \equiv G_{i}\left(G_{k} G_{l} G_{i^{\prime}} G_{i^{\prime \prime}}\right)$ vier harmonische Strahlen um den auf $p^{2}$ liegenden Punkt $G_{i}$ und mithin ihre zweiten Schnittpunkte $G_{k} G_{l} G_{i^{\prime}} G_{i^{\prime \prime}}$ mit $p^{2}$ vier harmonische Punkte auf $p^{2}$ sein. Folglich muß die $G_{k}$ mit $G_{l}$ verbindende Gerade $v_{k l}$ durch den Pol der $G_{i^{\prime}}$ mit $G_{i^{\prime \prime}}$ verbindenden Geraden in bezug auf $p^{2}$ gehen; der Pol der letzteren Verbindungsgeraden, der $\mathrm{zu} g_{i}$ in $(P)^{2} \mathrm{zu}-$ gepaarten Geraden (I in Nr. 54), muß aber, weil $(P)^{2}$ zugleich die Involution der in bezug auf $p^{2}$ konjugierten Strahlen um $P$ und $p$ die Polare von $P$ auch in bezug auf $p^{2}$ ist (Nr.2), der Schnittpunkt $Q_{i}$ von $p$ mit $g_{i}$ sein; mithin muß die $G_{k}$ mit $G_{l}$ verbindende Gerade $v_{k l}$ durch $Q_{i}$ gehen.

Geht, umgekehrt, die Gerade $v_{k l}$, welche zwei Punkte $G_{k}$ und $G_{l}$ von $p^{2}$ verbindet, durch den Punkt $Q_{i} \equiv g_{i} p$, den Pol der Geraden $G_{i^{\prime}} G_{i^{\prime \prime}}$ in bezug auf $p^{2}$, so sind $G_{k} G_{l} G_{i^{\prime}} G_{i^{\prime \prime}}$ vier harmonische Punkte auf $p^{2}$, die sie von dem gleichfalls auf $p^{2}$ liegenden Punkte $G_{i}$ aus projizierenden Strahlen $G_{i}\left(G_{k} G_{l} G_{i^{\prime}} G_{i^{\prime \prime}}\right)$ $\equiv v_{i k} v_{i l} v_{i^{\prime}} v_{i^{\prime \prime}}$ vier harmonische Strahlen um $G_{i}$ und deren Pole (in bezug auf $A B C$ ) $V_{i k} V_{i l} V_{i^{\prime}} V_{i^{\prime \prime}}$ vier harmonische Punkte auf $g_{i}^{2}$, und es muß also dann die Gerade $r$, welche $V_{i k}$ mit $V_{i l}$ verbindet, durch $P_{i}$, den $\mathrm{Pol}$ der $V_{i^{\prime}}$ mit $V_{i^{\prime \prime}}$ verbindenden Geraden in bezug auf $g_{i}^{2}$ (Nr.54), gehen. 
Nunmehr sind nach I in Nr. $50 G_{k}$ und $G_{l}$ die Pole derjenigen Geraden $g_{k}$ und $g_{l}$, welche die beiden weitern Schnittpunkte $P_{k}$ und $P_{l}$ der durch $P_{i}$ gehenden Geraden $r$ mit $p^{3}$ von $P$ aus projizieren; mithin ergibt sich:

II. Mit einem Punkte $P_{i}$ von $p^{3}$ liegen in je einer Geraden je zwei solche Punkte von $p^{3}$ und nur solche zwei Punkte, welche von $P$ aus durch solche zwei Geraden projiziert werden, deren beide Pole auf einer durch $Q_{i} \equiv p g_{i}$ gehenden Geraden liegen.

Also haben wir:

Satz 25. Drei Punkte von $p^{3}$ liegen dann und nur dann in einer Geraden, wenn die Pole (in bezug auf $A B C$ ) von irgend zwei und mithin von je zwei derjenigen drei Geraden, welche die drei Punkte von $p^{3}$ aus $P$ projizieren, mit dem Schnittpunkte der dritten Geraden und $p$, der Polare von $P$, in einer Geraden liegen.
Drei Strahlen von $P^{3}$ gehen dann und nur dann durch einen Punkt, wenn die Polaren (in bezug auf $A B C$ ) von irgend zwei und mithin von je zwei derjenigen drei Punkte, in welchen $p$ von den drei Strahlen von $P^{3}$ geschnitten wird, mit der den dritten Punkt mit $P$, dem Pole von $p$, verbindenden Geraden durch einen Punkt gehen.

56. Nun bilden die Pole der durch $Q_{i}$ gehenden Geraden den Kegelschnitt $q_{i}^{2}$, welcher letzte $A B C$ umschrieben ist und, weil $Q_{i}$ auf $p$ liegt, durch $P$ und, weil die Polaren $p$ und $p_{i}$ der beiden in $\left(g_{i}\right)^{2}$ ein Paar bildenden Punkte $P$ und $P_{i}$ auf $g_{i}$ sich schneiden müssen (Nr. 7) und also $Q_{i} \equiv p g_{i}$ auch auf $p_{i}$ liegt, durch $P_{i}$ geht. Folglich muß der Pol $V_{k l}$ der durch $Q_{i}$ gehenden, $G_{k}$ mit $G_{l}$ verbindenden Geraden $v_{k l}$, welcher Pol nach Hilfssatz 1 (Nr.47) auf der durch die beiden dem $P$ in $\left(g_{k}\right)^{2}$ und $\left(g_{l}\right)^{2}$ zupepaarten Punkte $P_{k}$ und $P_{l}$ und durch den $p^{3}$ und $q_{i}^{2}$ angehörenden Punkt $P_{i}$ gehenden Geraden $r$ liegen muß, der zweite Schnittpunkt von $r$ mit $q_{i}^{2}$ sein. Mithin haben wir nach Nr. 55:

III. Ist $P_{i}$ ein Punkt von $p^{3}, r$ irgendeine durch ihn gehende Gerade, $Q_{i}$ der Schnittpunkt der $P_{i}$ von $P$ aus projizierenden Geraden $g_{i}$ mit der Polare $p$ von $P, q_{i}^{2}$ der durch $P_{i}$ gehende Polarkegelschnitt von $Q_{i}, V_{k l}$ der zweite 
Schnittpunkt von $r$ mit $q_{i}^{2}$ und $v_{k l}$ dessen durch $Q_{i}$ gehende Polare, so hat $r$ mit $p^{3}$ noch diejenigen beiden Punkte $P_{k}$ und $P_{l}$ gemein und nur dienigen beiden, welche von $P$ aus durch $g_{k}$ und $g_{l}$, die Polaren der beiden Schnittpunkte $G_{k}$ und $G_{l}$ von $v_{k l}$ mit dem Polarkegelschnitt $p^{2}$ von $P$, projiziert werden. (Sind $G_{k}$ und $G_{l}$ konjugiert-imaginär, so müssen es auch nach der Anmerkung in Nr.51 $P_{k}$ und $P_{l}$ sein.)

\section{Aus dem Satze 25 ergibt sich, beiläufig bemerkt:}

Sind $G_{i}, G_{k}, G_{l}$ die Pole irgend dreier durch $P$ gehender Geraden $g_{i}, g_{k}, g_{l}$, und $Q_{i}, Q_{k}, Q_{l}$ die Schnittpunkte von $p$, der Polare von $P$, mit $g_{i}, g_{k}, g_{l}$ und geht $G_{i} G_{k}$ durch $Q_{l}$, so geht auch $G_{k} G_{l}$ durch $Q_{i}$ und $G_{l} G_{i}$ durch $Q_{k}$.

Es müssen nämlich die drei von $P$ aus durch $g_{i}, g_{k}, g_{l}$ projizierten Punkte $P_{i}, P_{k}, P_{l}$ von $p^{3}$ in einer Geraden liegen, weil $G_{i} G_{k}$ durch $Q_{l}$ geht, mithin müssen auch $G_{k} G_{l}$ und $G_{l} G_{i}$ durch $Q_{i}$ bzw. $Q_{k}$ gehen.

Hieraus folgt:

Die beiden nach (Nr.2) projektiven

Punktreihen erster und zweiter Ordnung, nämlich die von der Spur $Q_{x}$ eines um $P$ sich drehenden Strahles $g_{x}$ auf $p$, der Polare von $P$, beschriebene $p\left(Q_{x}\right)$ und die vom Pole $G_{x}$ von $g_{x}$ auf $p^{2}$, dem Polarkegelschnitt von $P$, beschriebene $p^{2}\left(G_{x}\right)$, haben die folgende eigentümliche Lage zueinander:

Sind $Q_{i}, G_{i}$ und $Q_{k}, G_{k}$ irgend zwei Paare entsprechender Punkte der projektiven Punktreihen $p\left(Q_{x}\right)$ und $p^{2}\left(G_{x}\right)$, so schneiden sich die beiden Verbindungs-
Strahlenbüschel erster und zweiter Ordnung, nämlich der von dem, einen auf $p$ sich bewegenden Punkt $Q_{x}$ von $P$, dem Pole von $p$, aus projizierenden Strahl $g_{x}$ beschriebene $P\left(g_{x}\right)$ und der von der Polare $q_{x}$ von $Q_{x}$ um $P^{2}$, den Polarkegelschnitt von $p$, beschriebene $P^{2}\left(q_{x}\right)$, haben die folgende eigentümliche Lage zueinander:

Sind $g_{i}, q_{i}$ und $g_{k}, q_{k}$ irgend zwei Paare entsprechender Strahlen der projektiven Büschel $P\left(g_{x}\right)$ und $P^{2}\left(q_{x}\right)$, so ist die Verbindungsgerade der beiden 
geraden $Q_{i} G_{k}$ und $Q_{k} G_{i}$ auf $p^{2}$, und zwar in demjenigen Punkte $G_{l}$, welcher dem Schnittpunkte $Q_{l}$ von $p$ mit $G_{i} G_{k}$ entspricht.

Aus jedem Punkte $G$ von $p^{2}$ und nur aus Punkten von $p^{2}$ werden die beiden projektiven Punktreihen $p\left(Q_{x}\right)$ und $p^{2}\left(G_{x}\right)$ durch involutorische Strahlenbüschel projiziert.
Schnittpunkte $g_{i} q_{k}$ und $g_{k} q_{i}$ eine Tangente an $P^{2}$, und zwar diejenige $q_{l}$, welche dem Verbindungsstrahle $g_{l}$ von $P$ mit $q_{i} q_{k}$ entspricht. Alle Tangenten von $P^{2}$ und nur diese Tangenten schneiden die beiden projektiven Büschel $P\left(g_{x}\right)$ und $P^{2}\left(q_{x}\right)$ in involutorischen Punktreihen.

Denn wenn irgendein Strahl durch $G p^{2}$ zum zweitenmal in $G_{i}$ und $p$ in $Q_{k}$ trifft, so geht auch $G_{k} Q_{i}$ durch $G$; diese beiden Strahlen $G_{i} Q_{k}$ und $G_{k} Q_{i}$ von $G$ projizieren sowohl $Q_{i}, G_{i}$ als auch $Q_{k}, G_{k}$ und entsprechen sich also in der Projektivität der beiden konzentrischen Büschel $G\left(Q_{x}\right)$ und $G\left(G_{x}\right)$ in beiderlei Sinne, diese beiden Büschel um $G$ sind folglich involutorisch. Und umgekehrt, wenn $p\left(Q_{x}\right)$ und $p^{2}\left(G_{x}\right)$ aus einem Punkte $G$ durch involutorische Büschel projiziert werden, so muß, wie man leicht einsehen kann, $G$ auf $p^{2}$ liegen.

58. Die Aussagen I (Nr. 54), II (Nr. 55) und III (Nr. 56) gelten auch dann noch, wenn $P_{i}$ eine der Ecken von $A B C$ ist.

Soll nämlich irgendeine durch die $p^{3}$ angehörende (Nr.46) Ecke $A$ gehende Gerade $r$ mit $p^{3}$ noch einen zweiten Punkt, etwa $\boldsymbol{P}_{k}$, und mithin (Nr.49 und Anmerkung in Nr. 51) noch einen dritten, etwa $P_{l}$, gemein haben (wo $P_{k}$ und $P_{l}$ von $P$ aus durch $g_{k}$ und $g_{l}$ projiziert werden), so wird der Pol $V_{k l}$ der Geraden $v_{k l}$, welche letzte die beiden Pole $G_{k}$ und $G_{l}$ von $g_{k}$ und $g_{l}$ verbindet, nach Hilfssatz 1 (Nr. 47) auf $r$ liegen müssen. Weil aber die beiden Geraden $V_{k l} P$ und $V_{k l} A \equiv r \equiv V_{k l} P_{k}$, die das Punktepaar $P P_{k}$ in $\left(g_{k}\right)^{2}$ von $V_{k l}$ aus projizieren, ein Strahlenpaar in der zu $\left(g_{k}\right)^{2}$ perspektiven (nach Satz 3 in Nr. 10) Strahleninvolution $\left(V_{k l}\right)^{2}$ bilden und also (Nr.4) voneinander durch $V_{k l} B$ und $V_{k l} C$ harmonisch getrennt sein müssen, so wird $V_{k l}$ auf dem von den sämtlichen Punkten, aus denen $P A B C$ durch vier harmonische Strahlen projiziert werden, gebildeten Kegelschnitt liegen müssen; dieser Kegelschnitt geht durch die vier Punkte $P A B C$ und wird in $A$ von der von $A P \equiv p_{A}$ durch $A B$ und $A C$ harmonisch ge- 
trennten Geraden $A P_{a}$ (Nr.2) tangiert und ist also mit dem Polarkegelschnitt $p_{a}^{\prime 2}$ des Schnittpunktes $P_{a}^{\prime}$ der Polare $p$ von $P$ mit der Geraden $p_{A} \equiv P A$ (Nr. 5) identisch, da auch dieser Polarkegelschnitt durch $A, B, C$ und, weil $P_{a}^{\prime}$ auf $p$ liegt, durch $P$ geht und in $A$ von der von $A P_{a}^{\prime} \equiv A P$ durch $A B$ und $A C$ harmonisch getrennten Geraden $A P_{a}$ tangiert wird (nach Nr.2). Mithin muß $V_{k l}$ der zweite Schnittpunkt der durch $A$ gehenden Geraden $r$ mit dem Polarkegelschnitt $p_{a}^{\prime 2}$ von $P_{a}^{\prime}$ sein, und die $G_{k}$ mit $G_{l}$ verbindende Gerade $v_{k l}$, die Polare von $V_{k l}$, muß durch $P_{a}^{\prime}$, den Schnittpunkt der Polare $p$ von $P$ mit der den $p^{3}$ angehörenden Eckpunkt $A$ von $P$ aus projizierenden Geraden $p_{A}$, gehen.

Auch wenn die durch $A$ gehende Gerade $r$ eine Dreieckseite, etwa $A B$, ist und also mit $p^{3}$ außer $A$ noch die Ecke $B$ und den Schnittpunkt $P_{c}$ von $A B$ mit $p$, der Polare von $P$, gemein hat (Nr.46), ist die Gerade, welche die beiden Pole der von $P$ aus $B$ und $P_{c}$ projizierenden Geraden $P B \equiv p_{B}$ und $P P_{c} \equiv p_{C}^{\prime}$ (Nr.5) verbindet, die durch $P_{a}^{\prime}$ gehende Polare des zweiten Schnittpunktes von $r \equiv A B$ mit dem Polarkegelschnitt $p_{a}^{\prime 2}$ von $P_{a}^{\prime}$. Denn der auf $p^{2}$ liegende $\mathrm{Pol}$ von $p_{C}^{\prime}$ ist (nach Satz 1 in Nr.7), weil $p_{C}^{\prime}$ zu $p_{C} \equiv P C$ in $(P)^{2}$ zugepaart ist (Nr.4) und der Pol von $p_{C}$ die auf dieser liegende Ecke $C$ ist, der zweite Schnittpunkt von $p^{2}$ mit $p_{C}$ und also, weil $p$ die Polare von $P$ auch in bezug auf $p^{2}$ ist (Nr.2), von $C$ durch $P$ und $P_{C}^{\prime}$, den Schnittpunkt von $p_{C}$ mit $p$ (Nr.5), harmonisch getrennt. Ferner ist der Pol von $p_{B} \equiv P B$ die Ecke $B$, welche von $C$ durch $(P A, a)$, den Schnittpunkt von $p_{A} \equiv P A$ mit der Seite $B C \equiv a$, und $P_{a}$, den Schnittpunkt von $a$ mit $p$, harmonisch getrennt ist (Nr.1). Nunmehr müssen diese beiden harmonischen Würfe auf $p_{C}$ und $a$, die $C$ gemein haben, in der Weise perspektiv liegen, daß die den Pol von $p_{C}^{\prime}$ mit $B$ verbindende Gerade durch den Schnittpunkt $P_{a}^{\prime}$ der beiden $P$ mit $(P A, a)$ und $P_{c}^{\prime}$ mit $P_{a}$ verbindenden Geraden $P A \equiv p_{A}$ und $p$ geht. Die Gerade, welche die beiden Pole von $p_{B}$ und $p_{C}^{\prime}$ verbindet, ist also mit der Geraden $P_{a}^{\prime} B$ identisch; die letztere Gerade ist aber die durch $P_{a}^{\prime}$ gehende Polare des zweiten Schnittpunktes, nämlich $B$, von $r \equiv A B$ mit $p_{a}^{\prime 2}$.

Geht, umgekehrt, die Gerade $v_{k l}$, welche zwei Punkte $G_{k}$ und $G_{l}$ von $p^{2}$ miteinander verbindet, durch den Punkt $P_{a}^{\prime} \equiv(p, P A)$, so liegt ihr Pol $V_{k l}$ auf dem Polarkegelschnitt $p_{a}^{\prime 2}$ von $P_{a}^{\prime}$; die die 
Ecke $A$ mit $V_{k l}$ verbindende Gerade $r$ wird dann, weil, wie wir sahen, $P A B C$ aus jedem der Punkte von $p_{a}^{\prime 2}$ durch vier harmonische Strahlen projiziert werden, nach Nr.4 zu der Geraden $V_{k l} P$ in $\left(V_{k l}\right)^{2}$ zugepaart sein müssen und folglich, weil $\left(V_{k l}\right)^{2}$ zu den Punktinvolutionen $\left(g_{k}\right)^{2}$ und $\left(g_{l}\right)^{2}$ auf den durch $P$ gehenden Polaren $g_{k}$ und $g_{l}$ von $G_{k}$ und $G_{l}$ perspektiv ist (nach Satz 3 in Nr. 10), durch die beiden dem $P$ in $\left(g_{k}\right)^{2}$ und $\left(g_{l}\right)^{2}$ zugepaarten und also $p^{3}$ angehörenden Punkte $P_{k}$ und $P_{l}$ gehen.

Mithin gelten die Aussagen II und III auch dann noch, wenn $P_{i}$ eine der Ecken von $A B C$ ist; denn, wenn $P_{i}$ etwa mit $A$ zusammenfallen soll, werden $g_{i}, Q_{i}$ und $q_{i}^{2}$ bzw. mit $p_{A} \equiv P A, P_{a}^{\prime}$ und $p_{a}^{\prime 2}$ zusammenfallen. Es gilt aber für die Ecken von $A B C$, wie wir bald sehen werden, auch die Aussage 1 in Nr. 54.

59. Aus III (Nr.56) folgt: Die durch $P_{i}$ gehende Gerade $r$ ist dann und nur dann Tangente an $p^{3}$ in $P_{k}$, wenn die durch $Q_{i}$ gehende Polare $v_{k l}$ des zweiten Schnittpunktes $V_{k l}$ von $r$ mit $q_{i}^{2}$ Tangente an $p^{2}$ ist. Denn dann und nur dann sind die beiden Schnittpunkte $G_{k}$ und $G_{l}$ von $v_{k l}$ mit $p^{2}$ und mithin auch ihre Polaren $g_{k}$ und $g_{l}$ und also auch $P_{k}$ und $P_{l}$ je einander unendlich nahe. Fällt $P_{i}$ etwa mit der Ecke $A$ zusammen, so fällt $Q_{i}$ mit $P_{a}^{\prime}$ zusammen, und die durch $A$ gehende Gerade $r$ ist also dann und nur dann Tangente an $p^{3}$ in $P_{k}$, wenn die durch $P_{a}^{\prime}$ gehende Polare $v_{k l}$ von $V_{k l}$, dem zweiten Schnittpunkte von $r$ mit $p_{a}^{\prime 2}$, Tangente an $p^{2}$ ist. Der Berührungspunkt der durch $P_{a}^{\prime}$ an $p^{2}$ gehenden Tangente $v_{k l}$, dessen Polare den Berührungspunkt der von $A$ an $p^{3}$ gehenden Tangente $r$ von $P$ aus projiziert, muß nun auf der Polare von $P_{a}^{\prime}$ in bezug auf $p^{2}$ liegen, welche Polare, weil $P_{a}^{\prime}$ der Schnittpunkt von $p$ mit $p_{A}$ ist und $p$ die Polare von $P$ auch in bezug auf $p^{2}$ und die Involution der in bezug auf $p^{2}$ konjugierten Strahlen um $P$ mit $(P)^{2}$ identisch ist (Nr. 2), die zu $p_{A}$, der $A$ von $P$ aus projizierenden Geraden, in $(P)^{2}$ zugepaarte Gerade $p_{A}^{\prime}$ sein muß; mithin gilt die Aussage I (Nr. 54) auch dann noch, wenn $P_{i}$ eine der Ecken von $A B C$ ist.

Die durch $P_{i}$ gehende Gerade $r$ ist ferner dann und nur dann Tangente an $p^{3}$ in demselben Punkte $P_{i}$, wenn die durch $Q_{i}$ gehende Polare $v_{k l}$ von $V_{k l}$ durch $G_{i}$ geht. Denn dann und nur dann ist einer der beiden Schnittpunkte $G_{k}$ und $G_{l}$ von $v_{k l}$ mit $p^{2}$, etwa $G_{k}$, von $G_{i}$ und mithin auch $g_{k}$ von $g_{i}$ und $P_{k}$ von 
$P_{i}$ nicht verschieden und $r$ hat alsdann $\mathrm{nur}$ noch einen von $P_{i}$ verschiedenen Punkt $P_{l}$ mit $p^{3}$ gemein. Fällt $P_{i}$ etwa mit $A$ und mithin $g_{i}$ mit $p_{A} \equiv P A, G_{i}$ wiederum mit $A, Q_{i}$ mit $P_{a}^{\prime}$ und $q_{i}^{2}$ mit $p_{a}^{\prime 2}$ zusammen, so ist die durch $A$ gehende Gerade $r$ dann und nur dann Tangente an $p^{3}$ in $A$, wenn die durch $P_{a}^{\prime}$ gehende Polare $v_{k l}$ von $V_{k l}$ gleichfalls durch $A$ geht und also mit $P_{a}^{\prime} A \equiv p_{A} \equiv P A$ zusammenfällt, was dann und nur dann der Fall ist, wenn $V_{k l}$, der zweite Schnittpunkt von $r$ mit $p_{a}^{\prime 2}$, von $A$, dem ersten Schnittpunkt von $r$ mit $p_{a}^{\prime 2}$, nicht verschieden ist, wenn also $r$ die Tangente $A P_{a}$ an $p_{a}^{\prime 2}$ in $A$ und somit die von $A P \equiv p_{A}$ durch $A B$ und $A C$ harmonisch getrennte Gerade ist (Nr.58). Mithin:

Die Tangente an $p^{3}$ in einer Ecke von $A B C$ ist von der durch diese Ecke und $P$ gehenden Ecktransversalen durch die beiden Dreieckseiten harmonisch getrennt. Die drei Punkte, in denen die Seiten von $A B C$ von den Tangenten an $p^{3}$ in den gegenüberliegenden Ecken geschnitten werden, sind die Schnittpunkte $P_{a}$, $P_{b}, P_{c}$ derselben Seiten mit der Polare $p$ von $P$.
Der Berührungspuknt von $P^{3}$ mit einer Seite von $A B C$ ist von dem Schnittpunkte dieser Seite mit $p$ durch die beiden Ecken harmonisch getrennt. Die drei Geraden, wolche die Ecken von $A B C$ mit den Berührungspunkten der gegenüberliegenden Seiten und $P^{3}$ verbinden, sind die durch den Pol $P$ von $p$ gehenden Ecktransversalen $p_{A}, p_{B}, p_{C}$ von $A B C$.

Ferner ist die durch $P_{i}$ gehende Gerade $r$ dann und nur dann eine Wendetangente an $p^{3}$, wenn die durch $Q_{i}$ gehende Polare $v_{k l}$ von $V_{k l}$ Tangente an $p^{2}$ in $G_{i}$ ist. Denn dann und nur dann sind die beiden Schnittpunkte $G_{k}$ und $G_{l}$ von $v_{k l}$ mit $p^{2}$ von $G_{i}$ und mithin auch $g_{k}$ und $g_{l}$ von $g_{i}$ und die beiden Punkte $P_{k}$ und $P_{l}$ von $P_{i}$ nicht verschieden und $r$ hat alsdann keinen von $P_{i}$ verschiedenen Punkt mit $p^{3}$ gemein. Endlich hat die Gerade $r$ drei voneinander verschiedene Punkte $P_{i}, P_{k}, P_{l}$ mit $p^{3}$ gemein, wenn die durch $Q_{i}$ gehende Polare $v_{k l}$ von $V_{k l}$ zwei voneinander und von $G_{i}$ verschiedene Punkte $G_{k}$ und $G_{l}$ mit $p^{2}$ gemein hat, wo $P_{k}$ und $P_{l}$ zugleich mit $G_{k}$ und $G_{l}$ reell oder imaginär sein müssen.

Hiernach geht das Kriterium des Satzes 23 (Nr.51) in folgendes über: 
Satz 26. Ist $P_{i}$ irgendein reeller Punkt von $p^{3}$ (mag er eine Ecke von $A B C$ sein oder nicht), $g_{i}$ die $P_{i}$ von $P$ aus projizierende Gerade, $G_{i}$ deren Pol, $Q_{i}$ der Schnittpunkt von $g_{i}$ mit der Polare $p$ von $P, q_{i}^{2}$ der Polarkegelschnitt von $Q_{i}$ (in bezug auf $A B C$ ), welcher Polarkegelschnitt durch $P_{i}$ geht, $p^{2}$ der durch $G_{i}$ gehende Polarkegelschnitt von $P$ (in bezug auf $A B C$ ), $r$ eine durch $P_{i}$ gehende reelle Gerade, $V_{k l}$ deren zweiter Schnittpunkt mit $q_{i}^{2}$ und $v_{k l}$ die durch $Q_{i}$ gehende Polare von $V_{k l}$, so sind die beiden weitern Schnittpunkte $P_{k}$ und $P_{l}$ von $r$ mit $p^{3}$ konjugiertimaginär, oder reell und voneinander und von $P_{i}$ verschieden, oder zwar voneinander, aber $P_{k}$ ist nicht von $P_{i}$ verschieden und $r$ ist also die Tangente an $p^{3}$ in $P_{i}$, oder $P_{k}$ und $P_{l}$ sind zwar von $P_{i}$, aber nicht voneinander verschieden und $r$ ist also die Tangente an $p^{3}$ in $P_{k}$, oder endlich ist weder $P_{k}$ noch $P_{l}$ von $P_{i}$ verschieden und $r$ ist also eine Wendetangente von $p^{3}$ in $P_{i}$, je nachdem $v_{k l}$ mit $p^{2}$ zwei konjugiert-imaginäre, oder zwei reelle, voneinander
Ist $p_{i}$ irgendein reeller Strahl von $P^{3}$ (mag er eine Seite von $A B C$ sein oder nicht), $Q_{i}$ der Punkt, in dem $p$ von $p_{i}$ geschnitten wird, $q_{i}$ dessen Polare, $g_{i}$ die $Q_{i}$ mit dem Pole $P$ von $p$ verbindende Gerade, $G_{i}{ }^{2}$ der Polarkegelschnitt von $g_{i}$ (in bezug auf $A B C$ ), welcher Polarkegelschnitt von $p_{i}$ tangiert wird, $P^{2}$ der von $q_{i}$ tangierte Polarkegelschnitt von $p$ (in bezug auf $A B C$ ), $N$ ein auf $p_{i}$ liegender reeller Punkt, $s_{m n}$ die zweite von $N$ an $G_{i}^{2}$ gehende Tangente (die erste ist nämlich $p_{i}$ ) und $S_{m n}$ der a uf $g_{i}$ liegende Pol von $s_{m n}$, so sind die beiden weitern durch $N$ gehenden Strahlen $p_{m}$ und $p_{n}$ von $P^{3}$ konjugiert-imaginär, oder reell und voneinander und von $p_{i}$ verschieden, oder zwar voneinander, aber $p_{m}$ ist nicht von $p_{i}$ verschieden und $N$ ist also der Berührungspunkt von $p_{i}$ mit $P^{3}$, oder $p_{m}$ und $p_{n}$ sind zwar von $p_{i}$, aber nicht voneinander verschieden und $N$ ist also der Berührungspunkt von $p_{m}$ mit $P^{3}$, oder endlich ist weder $p_{m}$ noch $p_{n}$ von $p_{i}$ verschieden und $N$ ist also ein Rückkehrpunkt von $P^{3}$ auf $p_{i}$, je nachdem von $S_{m n}$ zwei kon- 
und von $G_{i}$ verschiedene Punkte, oder $G_{i}$ und noch einen andern Punkt gemein hat, oder $v_{k l}$ Tangente an $p^{2}$ in einem von $G_{i}$ verschiedenen Punkte ist, oder endlich $v_{k l}$ die Tangente $t_{i}$ an $p^{2}$ in $G_{i}$ ist; und umgekehrt. jugiert-imaginäre, oder zwei reelle, voneinander und von $q_{i}$ verschiedene Tangenten, oder $q_{i}$ und noch eine andere Tangente an $P^{2}$ gehen, oder $S_{m n}$ Berührungspunkt in einer von $q_{i}$ verschiedenen Tangente von $P^{2}$ ist, oder endlich $S_{m n}$ der Berührungspunkt $U_{i}$ in der Tangente $q_{i}$ von $P^{2}$ ist; und umgekehrt.

Die Realität von $P_{i}$ und $r$ wurde deshalb vorausgesetzt, weil nur dann $P_{k}$ und $P_{l}$, wenn sie nicht reell sind, konjugiertimaginär sein müssen; sonst gilt aber der vorstehende Satz auch für imaginäre Punkte $P_{i}$ und Geraden $r$ (siehe Anmerkung in Nr. 51).

Eine Ausnahme hiervon macht nur die Gerade $g_{i} \equiv P_{i} P$. Der zweite Schnittpunkt von $g_{i}$ mit $q_{i}^{2}$ ist nämlich $P$ (nach Nr. 56 und 58) und seine Polare $p$, welche letzte mit $p^{2}$ zwei konjugiertimaginäre Punkte, nämlich die Doppelpunkte der von $A B C$ erzeugten elliptischen Involution $(p)^{2}$ (Nr. 2), gemein hat. Nun sind aber die Polaren der Doppelpunkte von $(p)^{2}$ die konjugiertimaginären Doppelstrahlen von $(P)^{2}$ (nach Nr. 3, 7 und 2), und die Schnittpunkte dieser Doppelstrahlen mit $g_{i}$ müssen also nach III die beiden weitern gemeinsamen Punkte von $g_{i}$ mit $p^{3}$ sein. Mithin müssen, weil $g_{i}$ von den beiden Doppelstrahlen von $(P)^{2}$ in einem und demselben reellen Punkte, nämlich in $P$, geschnitten wird, die beiden weitern gemeinsamen Punkte von $g_{i}$ und $p^{3}$, welche Punkte in $p^{3}$ von den konjugiert-imaginären Doppelstrahlen von $(P)^{2}$ herrühren, d. h. welche in den von $A B C$ auf diesen beiden Doppelstrahlen erzeugten Punktinvolutionen zweiten Grades $\operatorname{dem} P$ zugeordnet sind, in dem reellen Punkte $P$ vereinigt liegen.

Zugleich sehen wir, daß der Punkt $P$ sich selbst zugeordnet ist in jeder der beiden von $A B C$ auf den konjugiert-imaginären Doppelstrahlen von $(P)^{2}$ erzeugten Punktinvolutionen zweiten Grades, aber, wie aus Nr. 48 leicht zu folgern ist, auch nur in diesen beiden Punktinvolutionen; in der auf jeder andern durch $P$ gehenden Geraden von $A B C$ erzeugten Punktinvolution zweiten Grades wird dem Punkte $P$ ein von diesem verschiedener Punkt 
zugeordnet. Es liegt also auf jeder durch $P$ gehenden reellen oder imaginären, von den Doppelstrahlen von $(P)^{2}$ verschiedenen Geraden außer $P$ noch ein Punkt von $p^{3}$, während auf jedem der beiden konjugiert-imaginären Doppelstrahlen von $(P)^{2}$ außer $P$ mehr kein Punkt von $p^{3}$ liegt. Mithin:

Der Punkt $P$ ist ein isolierter Doppelpunkt von $p^{3}$ und die beiden Tangenten von $p^{3}$ in $P$, die beiden Doppelpunktstangenten, sind die konjugiertimaginären Doppelstrahlen von $(P)^{2}$. Außer $P$ besitzt $p^{3}$ mehr keine Doppelpunkte.
Die Gerade $p$ ist ein isolierter Doppelstrahl von $P^{3}$ und die beiden Berührungspunkte von $p$ mit $P^{3}$, die beiden Berührungspunkte des Doppelstrahles, sind die konjugiert-imaginären Doppelpunkte von $(p)^{2}$. Außer $p$ besitzt $P^{3}$ mehr keine Doppelstrahlen.

Das letzte ergibt sich auch aus der Bemerkung, daß durch jeden von $P$ verschiedenen Punkt und $P$ nur eine einzige Gerade geht und auf dieser gibt es nur einen einzigen Punkt, der dem $P$ zugepaart ist in der von $A B C$ auf dieser Geraiden erzeugten Involution zweiten Grades (siehe weiter unten Nr. 63).

60. Der letzte Fall des Satzes 26, nämlich, daß eine durch $P_{i}$ gehende Gerade $r$ eine Wendetangente an $p^{3}$ ist, kann nur dann eintreten, wenn $P_{i}$ einer der drei Schnittpunkte $P_{a}, P_{b}, P_{c}$ der Seiten von $A B C$ mit der Polare $p$ von $P$ ist. Denn in jenem Falle muß nach dem Satze 26 die Tangente $t_{i}$ an $p^{2}$ in $G_{i}$ durch $Q_{i}$, den Schnittpunkt von $g_{i}$ mit $p$, gehen, nach Satz 24 (Nr. 53) geht aber $t_{i}$ durch den auf $g_{i}$ liegenden Punkt $P_{i}$ von $p^{3}$; folglich wird dann der Punkt $P_{i}$ von $p^{3}$ mit $Q_{i}$ zusammenfallen und also auf $p$ liegen müssen, auf $p$ liegen aber keine anderen Punkte von $p^{3}$ als $P_{a}, P_{b}$ und $P_{c}$.

In jedem der drei Punkte $P_{a}, P_{b}, P_{c}$ gibt es aber wirklich eine Wendetangente von $p^{3}$. Denn, wenn $P_{i}$ etwa mit $P_{a}$ identisch ist, ist $g_{i}$ mit $P P_{a} \equiv p_{A}^{\prime}, G_{i}$ mit $P_{A}^{\prime}, Q_{i}$ mit $P_{a}$ und $q_{i}^{2}$ mit demjenigen Polarkegelschnitt $p_{a}^{2}$ des auf der Dreieckseite $a$ liegenden Punktes $P_{a}$ identisch, welcher Polarkegelschnitt in das aus der Seite $a$ und der Ecktransversale $p_{A} \equiv P A$ bestehende Geradenpaar ausartet (nach Nr.2). Nun ist aber $P_{A}^{\prime}$, der Pol von $p_{A}^{\prime}$, der zweite Schnittpunkt von $p_{A}$ mit $p^{2}$ (Nr.7) und die 
Tangente an $p^{2}$ in $P_{A}^{\prime}$ geht durch $P_{a}$ (nach Satz 24 in Nr. 53) und der Pol dieser Tangente muß also auf dem ausgearteten Polarkegelschnitt $p_{a}^{2}$ von $P_{a}$, und zwar auf $p_{A}$ liegen. Folglich wird die Gerade $r$, welche $\left(P_{i} \equiv\right) P_{a}$ mit dem auf $\left(q_{i}^{2} \equiv\right) p_{a}^{2}$ liegenden Pole der durch $\left(Q_{i} \equiv\right) P_{a}$ gehenden Tangente an $p^{2}$ in $\left(G_{i} \equiv\right) P_{A}^{\prime}$ verbindet, nach Satz 26 eine Wendetangente von $p^{3}$ in $\left(P_{i} \equiv\right) P_{a}$ sein müssen. Mithin:

Die drei Schnittpunkte $P_{a}, P_{b}, P_{c}$ der Seiten von $A B C$ mit der Polare $p$ von $P$ sind die einzigen Wendepunkte von $p^{3}$.
Die drei durch den Pol $P$ von $p$ gehenden Ecktransversalen $p_{A}, p_{B}, p_{C}$ von $A B C$ sind die einzigen Rückkehrstrahlen von $P^{3}$.

61. Aus I (Nr. 54), II (Nr. 55) und III (Nr. 56) ergeben sich unmittelbar folgende neue Lösungen der Aufgaben in Nr. 51, welche Lösungen auch im Falle, daß $P_{i}$ eine der Ecken von $A B C$ ist, anwendbar sind.

Auflösung 2 der Aufgabe 1. Man ermittelt zuerst den zweiten Schnittpunkt $V_{k l}$ von $r$ mit dem Polarkegelschnitt $q_{i}^{2}$ (der erste Schnittpunkt von $r$ mit $q_{i}^{2}$ ist nämlich $P_{i}$ ) von $Q_{i}$, dem Schnittpunkt von $g_{i}$ mit der Polare $p$ von $P$, und dessen durch $Q_{i}$ gehende Polare $v_{k l}$ (in bezug auf $A B C$ ), sodann die beiden Schnittpunkte $G_{k}$ und $G_{l}$ von $v_{k l}$ mit dem Polarkegelschnitt $p^{2}$ von $P$ und ihre durch $P$ gehenden Polaren $g_{k}$ und $g_{l}$ (in bezug auf $A B C$ ); alsdann sind die beiden Schnittpunkte $P_{k}$ und $P_{l}$ von $r$ mit $g_{k}$ und $g_{l}$ die gesuchten Schnittpunkte von $r$ und $p^{3}$.

Diese Lösung liefert auch dann noch die Punkte $P_{k}$ und $P_{l}$, wenı $G_{k}$ und $G_{l}$ imaginär sind (siehe Anmerkung in Nr.51).

Aufgabe 4. Auf einer durch zwei Punkte $P_{k}$ und $P_{l}$ von $p^{3}$ gehenden Geraden $r$, wo $P_{k}$ und $P_{l}$ von $P$ aus durch die Geraden $g_{k}$ und $g_{l}$ projiziert werden, den dritten Schnittpunkt mit $p^{3} \mathrm{zu}$ ermitteln.

Auflösung 1. Man ermittelt den Schnittpunkt $Q_{k}$ von $g_{k}$ mit $p$, der Polare von $P$, und den auf $p^{2}$, dem Polarkegelschnitt von $P$, liegenden $\mathrm{Pol} G_{l}$ von $g_{l}$, sodann den zweiten Schnittpunkt $G_{i}$ von $Q_{k} G_{l}$ mit $p^{2}$ und dessen Polare Burliner, Habilitationsschrift. 
$g_{i}$ (in bezug auf $A B C$ ); alsdann ist der Schnittpunkt $P_{i}$ von $g_{i}$ mit $r$ der gesuchte dritte Schnittpunkt von $r$ mit $p^{3}$.

Auflösung 2. Man ermittelt die beiden Pole $G_{k}$ und $G_{l}$ von $g_{k}$ und $g_{l}$, bringt die $G_{k}$ mit $G_{l}$ verbindende Gerade zum Schnitt mit der Polare $p$ von $P$ und verbindet diesen Schnittpunkt mit $P$ durch eine Gerade $g_{i}$; alsdann ist der Schnittpunkt $P_{i}$ von $r$ mit $g_{i}$ der gesuchte dritte Schnittpunkt von $r$ mit $p^{3}$.

Diese beiden Lösungen folgen unmittelbar aus II und dienen auch zur Lösung der nächstfolgenden Aufgabe, wenn man beachtet, daß eine Tangente an einer Kurve in ihrem Berührungspunkte zwei einander unendlich nahe liegende Punkte der Kurve enthält.

Aufgabe 5. Auf der Tangente $t_{k}$ an $p^{3}$ in einem Punkte $P_{k}$ (wo $P_{k}$ von $P$ aus durch $g_{k}$ projiziert wird) den weitern Schnittpunkt mit $p^{3}$, den Tangentialpunkt von $P_{k}$, $\mathrm{zu}$ ermitteln.

Auflösung 1. Man ermittelt den Pol $G_{k}$ von $g_{k}$, welcher Pol auf dem Polarkegelschnitt $p^{2}$ von $P$ liegt, sodann die Tangente $t_{k}$ an $p^{2}$ in $G_{k}$, bringt diese zum Schnitt mit der Polare $p$ von $P$ und verbindet den Schnittpunkt $p t_{k}$ mit $P$ durch eine Gerade; alsdann ist der Schnittpunkt der letzten Geraden mit $\mathrm{t}_{k}$ der gesuchte Tangentialpunkt von $P_{k}$.

Auflösung 2. Man ermittelt den Schnittpunkt $Q_{k}$ von $g_{k}$ mit $p$, der Polare von $P$, und den auf $p^{2}$, dem Polarkegelschnitt von $P$, liegenden $\mathrm{Pol} G_{k}$ von $g_{k}$, sodann den zweiten Schnittpunkt von $Q_{k} G_{k}$ mit $p^{2}$ und dessen Polare (in bezug auf $A B C$ ); alsdann ist der Schnittpunkt dieser Polare mit $\mathrm{t}_{k}$ der gesuchte Tangentialpunkt von $P_{k}$.

Auflösung 3. Man ermittelt den $\operatorname{Pol} G_{k}$ von $g_{k}$, sodann diejenige Gerade, welche in der von $A B C$ erzeugten Strahleninvolution $(P)^{2}$ zu der $G_{k}$ mit $P$ verbindenden Geraden, dem ersten Repräsentanten $g_{k}^{(1)}$ von $g_{k}$ (Nr. 24), zugepaart ist; alsdann ist der Schnittpunkt dieser Geraden mit $t_{k}$ der gesuchte Tangentialpunkt von $P_{k}$.

Die dritte Lösung folgt aus der Aussage I (Nr.54); denn nach dieser Aussage muß der Tangentialpunkt $P_{i}$ irgendeines Punktes $P_{i^{\prime}}$ von $p^{3}$ (wo $P_{i^{\prime}}$ von $P$ aus durch $g_{i^{\prime}}$ projiziert wird) von $P$ aus durch diejenige Gerade $g_{i}$ projiziert werden, welche 
in $(P)^{2}$ zu der den Pol $G_{i^{\prime}}$ von $g_{i^{\prime}}$ mit $P$ verbindenden Geraden, dem ersten Repräsentanten $g_{i^{\prime}}^{(1)}$ von $g_{i^{\prime}}$, zugepaart ist; mithin muß der Schnittpunkt der Tangente an $p^{3}$ in $P_{i^{\prime}}$ mit $g_{i} P_{i}$ sein; auch erhellt diese Lösung aus der ersten, wie man einsehen kann.

Zugleich sehen wir, daß, wenn in jeder der drei Lösungen der Aufgabe 5 an Stelle des Schnittpunktes der letzt zu ermittelnden Geraden mit $\mathrm{t}_{k}$ derjenige Punkt ermittelt wird, der zu $P$ zugepaart ist in der von $A B C$ auf jener letzten Geraden erzeugten Involution zweiten Gerades, dieser Punkt der Tangentialpunkt von $P_{k}$ sein wird; damit haben wir drei Lösungen der

Aufgabe 5a. Den Tangentialpunkt eines Punktes $P_{k}$ von $p^{3}$ zu ermitteln, wenn weder $P_{k}$ selbst noch die Tangente $t_{k}$ in ihm gegeben sind, sondern die ihn von $P$ aus projizierende Gerade $g_{k}$.

Aus dem Satze 26 ergibt sich die folgende

Auflösung 2 der Aufgabe 2 (Nr.51). Man ermittelt die beiden von $Q_{i}$, dem Schnittpunkte von $g_{i}$ mit $p$, an $p^{2}$ gehenden Tangenten, sodann die Pole dieser beiden Tangenten (in bezug auf $A B C$ ); alsdann sind die beiden diese Pole mit $P_{i}$ verbindenden Geraden und nur diese beiden die gesuchten Tangenten an $p^{3}$.

Es gehen also aus jedem beliebigen Punkte $P_{i}$ von $p^{3}$ (die Ecken von $A B C$ nicht ausgenommen) zwei und nur zwei Tangenten (außer der Tangente in $P_{i}$ selbst) an $p^{3}$.

Auflösung 3 der Aufgabe 2. Man ermittelt die zu $g_{i}$ in $(P)^{2}$ zugepaarte Gerade und ihre beiden Schnittpunkte mit $p^{2}$, sodann die durch $P$ gehenden Polaren (in bezug auf $A B C$ ) der beiden Schnittpunkte und in den von $A B C$ auf diesen Polaren erzeugten Punktinvolutionen zweiten Grades die beiden dem $P$ zugepaarten Punkte; alsdann sind die beiden $P_{i}$ mit den letzten Punkten verbindenden Geraden die gesuchten Tangenten an $p^{3}$ und jene letzten Punkte ihre Berührungspunkte.

Diese Lösung folgt unmittelbar aus I (Nr.54) und liefert, wenn $P_{i}$ einer der drei Wendepunkte $P_{a}, P_{b}, P_{c}$ von $p^{3}$ (Nr.60) ist, nur eine der beiden gesuchten Tangenten (die andere fällt alsdann mit der Wendetangente in $P_{i}$ zusammen), sonst beide. 
Auflösung 2 der Aufgabe 3 (Nr.51). Man ermittelt den Pol $G_{i}$ von $g_{i}$, sodann den Pol der $G_{i}$ mit $Q_{i}$, dem Schnittpunkte von $g_{i}$ mit $p$, verbindenden Geraden; alsdann ist die den letztern $\mathrm{Pol}_{\text {mit }} P_{i}$ verbindende Gerade die Tangente von $p^{3}$ in $P_{i}$.

Diese Lösung ergibt sich unmittelbar aus dem Satze 26.

Auflösung 3 der Aufgabe 3. Man ermittelt den Pol $G_{i}$ von $g_{i}$, sodann diejenige Gerade, welche in $(P)^{2}$ zu der $G_{i}$ mit $P$ verbindenden Geraden, dem ersten Repräsentanten $g_{i}^{(1)}$ von $g_{i}$, zugepaart ist, und in der von $A B C$ auf dieser Geraden erzeugten Punktinvolution zweiten Grades den zu $P$ zugepaarten Punkt; alsdann ist dieser letzte Punkt der Tangentialpunkt von $P_{i}$ und die ihn mit $P_{i}$ verbindende Gerade die Tangente von $p^{3}$ in $P_{i}$.

Diese Lösung folgt aus I in Nr. 54 (s. oben Auflösung 3 der Aufgabe 5) und ist nur dann anzuwenden, wenn $P_{i}$ keiner der Wendepunkte $P_{a}, P_{b}, P_{c}$ von $p^{3}$ ist; ist aber $P_{i}$ einer dieser drei Wendepunkte, so fällt der letzte Punkt, der Tangentialpunkt von $P_{i}$, mit $P_{i}$ zusammen und die Wendetangente in $P_{i}$ kann hierdurch nicht ermittelt werden; es ist also dann vielmehr die vorhergehende Lösung 2 oder die Lösung der Aufgabe 3 in Nr. ó1 anzuwenden, wo dann die Tangente $t_{i}$ an $p^{2}$ in $G_{i}$ die $G_{i}$ mit $P_{i}$ verbindende Gerade sein wird.

Anmerkung. Mit Hilfe der Ordinatenwinkel (Nr. 26) kann man in den Auflösungen 3 der Aufgaben 3 und 5 die in $(P)^{2}$ zu $g_{i}^{(1)}$ zugepaarte Gerade direkt, ohne Vermittelung des Poles $G_{i}$, bestimmen: ist nämlich $\omega_{i}$ der Ordinatenwinkel von $g_{i}$, so ist der Ordinatenwinkel von $g_{i}^{(1)}$ gleich $-2 \omega_{i}$ (Satz 15 in Nr. 26) und der von der zu $g_{i}^{(1)}$ in $(P)^{2}$ zugepaarten Geraden gleich $-2 \omega_{i} \pm \frac{\pi}{2} ;$ ferner sind in der Auflösung 3 der Aufgabe 2 $-\left(\frac{\omega_{i}}{2}+\frac{\pi}{4}\right)$ und $-\left(\frac{\omega_{i}}{2}-\frac{\pi}{4}\right)$ die Ordinatenwinkel der beiden durch $P$ gehenden Polaren der Schnittpunkte von $p^{2}$ mit der zu $g_{i}$ in $(P)^{2}$ zugepaarten Geraden.

Aufgabe 6. Die durch zwei Punkte $P_{i}$ und $P_{k}$ von $p^{3}$ gehende Gerade zu ziehen, wenn $P_{i}$ und $P_{k}$ selbst nicht gegeben sind, sondern die sie von $P$ aus projizierenden Geraden $g_{i}$ und $g_{k}$. 
Auflösung. Man ermittelt die Pole $G_{i}$ und $G_{k}$ von $g$ und $g_{k}$ und etwa den Schnittpunkt $Q_{i}$ von $g_{i}$ mit $p$, sodann die beiden Pole von $G_{i} G_{k}$ und $Q_{i} G_{k}$; alsdann wird die die letztern beiden Pole verbindende Gerade die gesuchte sein und ihre Schnittpunkte mit $g_{i}$ und $g_{k}$ werden die Punkte $P_{i}$ und $P_{k}$ sein.

Denn ist etwa $P_{l}$ der dritte Schnittpunkt von $P_{i} P_{k}$ mit $p^{3}$, so müssen nach Hilfssatz 1 (Nr. 47) die drei Pole von $G_{i} G_{k}, G_{k} G_{l}$, $G_{l} G_{i}$ auf $P_{i} P_{k}$ liegen; $G_{k} G_{l}$ ist aber mit $Q_{i} G_{k}$ identisch und $G_{l} G_{i}$ mit $Q_{k} G_{i}$ (Nr.57). $P_{i} P_{k}$ ist also durch zwei jener drei Pole bestimmt.

A ufgabe 7. Die Tangente in einem Punkte $P_{\imath}$ von $p^{3}$ zu ziehen, wenn $P_{i}$ selbst nicht gegeben ist, sondern die ihn von $P$ aus projizierende Gerade $g_{i}$.

Auflösung. Man ermittelt den Pol $G_{i}$ von $g_{i}$ und die Tangente $t_{i}$ an $p^{2}$, dem Polarkegelschnitt von $P$, in $G_{i}$, sodann die beiden Pole (in bezug auf $A B C$ ) von $t_{i}$ und $G_{i} Q_{i}$ $\left(Q_{i} \equiv p g_{i}\right)$; alsdann wird die diese beiden Pole verbindende Gerade die gesuchte Tangente sein und ihr Schnittpunkt mit $g_{i}$ ihr Berührungspunkt $P_{i}$ mit $p^{3}$.

Denn ist etwa $P_{n}$ der Tangentialpunkt von $P_{i}$, geht also die Tangente $t_{i}$ an $p^{3}$ in $P_{i}$ auch durch $P_{n}$, so muß nach Satz 26 der zweite Schnittpunkt von $\mathrm{t}_{i}$ mit $q_{i}^{2}$, dem Polarkegelschnitt - von $Q_{i}$, der Pol von $Q_{i} G_{i}$ sein und der zweite Schnittpunkt von $\mathrm{t}_{i}$ mit $q_{n}^{2}$, dem Polarkegelschnitt von $Q_{n} \equiv p g_{n}$, der Pol der durch $Q_{n}$ gehenden Tangente $t_{i}$ von $p^{2}$ in $G_{i}$.

Diese Lösung versagt nur dann, wenn $P_{i}$ ein Wendepunkt von $p^{3}$ ist, wenn also (Nr.60) $t_{i}$ mit $Q_{i} G_{i}$ zusammenfällt; alsdann aber genügt es, den Pol von $t_{i} \equiv Q_{i} G_{i}$ mit $Q_{i}$ zu verbinden (wo dann $Q_{i}$ mit dem Wendepunkte $P_{i}$ identisch ist) und diese Verbindungsgerade wird die Wendetangente in $P_{i}$ sein.

Aufgabe 8. Die beiden aus einem Punkte $P_{i}$ von $p^{3}$ an diese gehenden Tangenten zu ziehen, wenn nur $g_{i}$, nicht aber $P_{i}$ gegeben ist.

Auflösung. Man ermittelt die beiden aus $Q_{i} \equiv p g_{i}$ an $p^{2}$, den Polarkegelschnitt von $P$, gehenden Tangenten $t_{i^{\prime}}$ und $t_{i^{\prime \prime}}$, ihre Berührungspunkte $G_{i^{\prime}}$ und $G_{i^{\prime \prime}}$ und den Pol $G_{i}$ von $g_{i}$ (in bezug auf $A B C$ ), sodann die Pole (in bezug auf $A B C$ ) 
von $t_{i^{\prime}}, t_{i^{\prime \prime}}, G_{i^{\prime}} G_{i}$ und $G_{i^{\prime \prime}} G_{i}$; alsdann werden die die Pole von $t_{i^{\prime}}$ und $G_{i} G_{i^{\prime}}$ und die die Pole von $t_{i^{\prime \prime}}$ und $G_{i} G_{i^{\prime \prime}}$ verbindenden Geraden die beiden gesuchten Tangenten sein.

Diese Lösung ergibt sich aus dem Satze 26 ganz analog wie die vorige.

Alle entsprechenden Aufgaben für das duale Gebilde $P^{3}$ sind dual zu lösen.

62. Ist, wie im Satze 26 (Nr.59) angenommen wurde, der Punkt $P_{i}$ von $p^{3}$ und mithin auch $Q_{i}$, der Schnittpunkt von $g_{i}$ mit der gänzlich außerhalb des Polarkegelschnitts $p^{2}$ von $P$ verlaufenden Polare $p$ von $P$ (Nr.2), reell, so gehen von $Q_{i}$ zwei reelle Tangenten an $p^{2}$; durch diese beiden Tangenten werden die durch $Q_{i}$ gehenden und stetig aufeinanderfolgenden Geraden $v$, welche mit $p^{2}$ zwei konjugiert-imaginäre Punkte gemein haben, von den durch $Q_{i}$ gehenden und gleichfalls stetig aufeinanderfolgenden Geraden $v$, welche mit $p^{2}$ zwei reelle Punkte gemein haben, getrennt. Weil nun der Strahlenbüschel der Polaren $v$ um $Q_{i}$ zu der Punktreihe der Pole $V$ auf dem Polarkegelschnitt $q_{i}^{2}$ von $Q^{i}$ projektiv ist, so wird jeder stetigen Aufeinanderfolge von Polaren $v$ um $Q_{i}$ eine stetige Aufeinanderfolge von Polen $V$ auf $q_{i}^{2}$ entsprechen und diese wird von dem auf $p^{3}$ und $q_{i}^{2}$ liegenden Punkte $P_{i}$ aus durch eine stetige Aufeinanderfolge von Geraden $r$ projiziert. Demnach werden die durch $P_{i}$ gehenden Geraden $r$, welche $q_{i}^{2}$ zum zweitenmal in solchen Punkten $V$ schneiden, welche Pole der durch $Q_{i}$ gehenden und $p^{2}$ in je zwei konjugiert-imaginären Punkten schneidenden Geraden $v$ sind, stetig aufeinander folgen und ebenso die durch $P_{i}$ gehenden Geraden $r$, welche $q_{i}^{2}$ zum zweitenmal in solchen Punkten $V$ schneiden, welche Pole der durch $Q_{i}$ gehenden und $p^{2}$ in je zwei reellen Punkten schneidenden Geraden $v$ sind; und die erstern Geraden $r$ werden von den letztern durch diejenigen beiden Geraden von $P_{i}$ getrennt, welche $q_{i}^{2}$ zum zweitenmal in den Polen der beiden von $Q_{i}$ an $p^{2}$ gehenden Tangenten schneiden. Unter den erstern Geraden $r$ befindet sich die Gerade $g_{i} \equiv P_{i} P$, da die Polare $p$ des zweiten Schnittpunktes $P$ von $g_{i}$ mit $q_{i}^{2}$ (Nr. 56 und 58) ganz außerhalb $p^{2}$ verläuft. Mithin haben wir nach Satz 26:

Von jedem $p^{3}$ angehörenden reellen Punkte $P_{i}$ (mag

Auf jedem $P^{3}$ angehörenden reellen Strahle $p_{i}$ (mag 
dieser eine Ecke von $A B C$ sein oder nicht) gehen außer der Tangente in $P_{i}$ selbst nur noch zwei reelle Tangenten an $p^{3}$, von denen eine, wenn $P_{i}$ einer der drei Wendepunkte $P_{a}, P_{b}, P_{c}$ von $p^{3}$ ist, mit der Wendetangente in $P_{i}$ zusammenfällt (Nr. 60). In dem einen der beiden von diesen zwei Tangenten um $P_{i}$ gebildeten vollkommenen Winkel, und zwar in dem, innerhalb dessen $P$, der isolierte Doppelpunkt von $p^{3}$ (Ende Nr. 59), nicht liegt, sind diejenigen und nur diejenigen durch $P_{i}$ gehenden Geraden enthalten, welche mit $p^{3}$ außer $P_{i}$ noch je zwei reelle Punkte gemein haben, und in dem zweiten Winkel sind diejenigen und nur diejenigen durch $P_{i}$ gehenden Geraden enthalten, welche mit $p^{3}$ außer $P_{i}$ mehr keinen reellen Punkt gemein haben. Die Kurve $p^{3}$ ist also gänzlich in dem einen der beiden vollkommenen Winkel enthalten, die von zwei aus einem beliebigen $p^{3} \quad$ angehörenden Punkte an $p^{3}$ gehenden Tangenten gebildet werden, und zwar in demjenigen Winkel, innerhalb dessen $P$ nicht liegt. dieser eine Seite von $A B C$ sein oder nicht) liegen außer dem Berührungspunkt von $P^{3}$ mit $p_{i}$ selbst nur noch zwei reelle Berührungspunkte von $P^{3}$, von denen einer, wenn $p_{i}$ einer der drei Rückkehrstrahlen $p_{A}, p_{B}, p_{C}$ von $P^{3}$ ist, mit dem Rückkehrpunkte auf $p_{i}$ zusammenfällt. Auf der einen der beiden von diesen zwei Berührungspunkten auf $p_{i}$ begrenzten Strecken, und zwar auf der, die von $p$, dem isolierten Doppelstrahle von $P^{3}$, nicht getroffen wird, liegen diejenigen und nur diejenigen Punkte, durch welche außer $p_{i}$ noch je zwei reelle Strahlen von $P^{3}$ gehen, und auf der zweiten Strecke liegen diejenigen und nur diejenigen Punkte, durch welche außer $p_{i}$ mehr kein reeller Strahl von $P^{3}$ geht. Jeder Strahl des Büschels $P^{3}$ wird also von den sämtlichen Strahlen von $P^{3}$ in der einen der beiden Strecken getroffen, die auf ihm von den zwei Berührungspunkten von $P^{3}$ (außer dem Berührungspunkt mit ihm selbst) begrenzt werden, und zwar in derjenigen Strecke, die von $p$ nicht getroffen wird. 
63. Aus den Aussagen I (Nr. 54), II (Nr. 55) und III (Nr. 56) läßt sich nun eine Reihe bekannter Sätze über Kurven dritter Ordnung für unsere Kurve $p^{3}$ von neuem ableiten.

Nach II bilden die Pole je zweier Geraden, welche letzte von $P$ aus zwei mit einem Punkte $P_{i}$ von $p^{3}$ in einer und derselben Geraden liegende Punkte von $p^{3}$ projizieren, auf dem Polarkegelschnitt $p^{2}$ von $P$ eine hyperbolische Punktinvolution, deren Zentrum der außerhalb $p^{2}$ liegende (Nr.62) Punkt $Q_{i} \equiv g_{i} p$ ist und deren Doppelpunkte $G_{i^{\prime}}$ und $G_{i^{\prime \prime}}$, die Pole der die Berührungspunkte $P_{i^{\prime}}$ und $P_{i^{\prime \prime}}$ der beiden von $P_{i}$ an $p^{3}$ gehenden Tangenten von $P$ aus projizierenden und ein Strahlenpaar in $(P)^{2}$ bildenden Geraden $g_{i^{\prime}}$ und $g_{i^{\prime \prime}}$, sind und auf der zu $g_{i}$ in $(P)^{2}$ zugepaarten Geraden liegen ( $\mathrm{I}$ in Nr.54). Nun ist der Büschel der Polaren um $P$ zu der Punktreihe der Pole auf $p^{2}$ projektiv (Nr. 2); mithin ergibt sich:

Die Punktepaare auf $p^{3}$, welche mit einem von $P$, dem isolierten Doppelpunkte von $p^{3}$, aus durch die Gerade $g_{i}$ projizierten Punkte $P_{i}$ in je einer Geraden liegen, werden von $P$ aus durch Strahlenpaare einer auf $(P)^{2}$ sich stützenden Involution projiziert, deren Doppelstrahlen dasjenige Strahlenpaar in $(P)^{2}$ bilden, welches die Berührungspunkte der beiden von $P_{i}$ an $p^{3}$ gehenden Tangenten projiziert und dessen Pole mithin auf der zu $g_{i}$ in $(P)^{2}$ zugepaarten Geraden liegen, welches Strahlenpaar also (Nr.8) die letzte Gerade zu ihrem Repräsentanten hat; und umgekehrt. Dabei wird der Tangentialpunkt von $P_{i}$ durch denjenigen Strahl von $P$ aus projiziert, welcher in der auf $(P)^{2}$ sich stützenden Involution zu $g_{i}$ zugepaart ist.

Denn auf der $P_{i}$ mit seinem Tangentialpunkte verbindenden Geraden, also auf der Tangente von $p^{3}$ in $P_{i}$, liegt kein weiterer Punkt von $p^{3}$.

Weil in jeder auf $(P)^{2}$ sich stützenden Involution die Doppelstrahlen von $(P)^{2}$ ein Paar bilden, so muß nun dasjenige Punktepaar auf $p^{3}$, welches von $P$ aus durch die Doppelstrahlen von $(P)^{2}$ projiziert wird, mit jedem beliebigen Punkte von $p^{3}$ in einer Geraden liegen; dieses Punktepaar muß folglich in einem einzigen Punkte und also in dem Schnittpunkte $P$ der Doppelstrahlen von $(P)^{2}$ zusammenfallen, da sonst alle Punkte 
von $p^{3}$ auf der dieses Punktepaar verbindenden Geraden liegen müßten. $P$ ist also, wie schon oben (Ende Nr. 59) bewiesen wurde, ein Doppelpunkt von $p^{3}$, und zwar der einzige Doppelpunkt, da die Doppelstrahlen von $(P)^{2}$ die einzigen sind, die in den sämtlichen auf $(P)^{2}$ sich stützenden Involutionen ein Paar bilden.

Weil ferner jedes Strahlenpaar in einer Involution durch deren Doppelstrahlen harmonisch getrennt wird, so ergibt sich:

Auf jeder durch einen Punkt $P_{i}$ von $p^{3}$ gehenden Geraden werden die beiden weitern Schnittpunkte mit $p^{3}$ durch die beiden Geraden, welche von $P$, dem isolierten Doppelpunkte von $p^{3}$, aus die Berührungspunkte der beiden von $P_{i}$ an $p^{3}$ gehenden Tangenten projizieren, harmonisch getrennt.

64. Verstehen wir unter dem Ordinatenwinkel eines Punktes $P_{i}$ von $p^{3}$ den Ordinatenwinkel $\omega_{i}$ des diesen Punkt von $P$ aus projizierenden Strahles $g_{i}(\mathrm{Nr} .26)$, so ergibt sich hieraus die folgende lineare Relation zwischen den Ordinatenwinkeln dreier in einer Geraden liegender Punkte von $p^{3}$.

Satz 27. Drei Punkte $P_{i}, P_{k}, P_{l}$ von $p^{3}$ liegen dann und nur dann in einer Geraden, wenn ihre Ordinatenwinkel der Forderung genügen :

$$
\omega_{i}+\omega_{k}+\omega_{l}=\frac{2 k+1}{2} \pi,
$$

wo $k$ eine beliebige ganze Zahl bedeutet.

Beweis. Sind $g_{i}, g_{k}, g_{l}$ die $P_{i}, P_{k}, P_{l}$ von $P$ aus projizierenden Strahlen, $g_{i}^{\prime}$ der zu $g_{i}$ in $(P)^{2}$ zugepaarte Strahl, $g_{i^{\prime}}$ und $g_{i^{\prime \prime}}$ die die Berührungspunkte $P_{i^{\prime}}$ und $P_{i^{\prime \prime}}$ der beiden von $P_{i}$ an $p^{3}$ gehenden Tangenten von $P$ aus projizierenden Strahlen, deren Repräsentant, wie wir sahen, $g_{i}^{\prime}$ ist, und sind also $\omega_{i}, \omega_{k}, \omega_{l}, \omega_{i}^{\prime}$, $\omega_{i^{\prime}}, \omega_{i^{\prime}}$ der Reihe nach die Ordinatenwinkel von $g_{i}, g_{k}, g_{l}, g_{i}^{\prime}, g_{i^{\prime}}, g_{i^{\prime \prime}}$, so ist $\omega_{i}^{\prime}=\omega_{i}+\frac{\pi}{2}$ und nach Satz $15(\mathrm{Nr} .26) \omega_{i^{\prime}}=-\left(\omega_{i}+\frac{\pi}{2}\right): 2$ $=-\left(\frac{\omega_{i}}{2}+\frac{\pi}{4}\right)$ und $\omega_{i^{\prime \prime}}=-\left(\frac{\omega_{i}}{2}+\frac{\pi}{4}\right)+\frac{\pi}{2}=-\left(\frac{\omega_{i}}{2}-\frac{\pi}{4}\right)$. Weil aber, wie wir sahen, $\boldsymbol{P}_{i}, P_{k}, P_{l}$ dann und nur dann in einer Geraden liegen, wenn $g_{k} g_{l} g_{i^{\prime}} g_{i^{\prime \prime}}$ vier harmonische Strahlen sind 
und mithin auch die ihre Spuren auf $p$ von $R$, dem Scheitel der Ordinatenwinkel (Nr.26), aus projizierenden Geraden, so ist jenes dann und nur dann der Fall, wenn

also:

$$
\frac{\sin \left(\omega_{k}-\omega_{i^{\prime}}\right)}{\sin \left(\omega_{k}-\omega_{i^{\prime \prime}}\right)}: \frac{\sin \left(\omega_{l}-\omega_{i^{\prime}}\right)}{\sin \left(\omega_{l}-\omega_{i^{\prime \prime}}\right)}=-1,
$$

$$
\begin{gathered}
\frac{\sin \left(\omega_{k}+\frac{\omega_{i}}{2}+\frac{\pi}{4}\right)}{\sin \left(\omega_{k}+\frac{\omega_{i}}{2}-\frac{\pi}{4}\right)}: \frac{\sin \left(\omega_{l}+\frac{\omega_{i}}{2}+\frac{\pi}{4}\right)}{\sin \left(\omega_{l}+\frac{\omega_{i}}{2}-\frac{\pi}{4}\right)} \\
=\operatorname{tg}\left(\omega_{k}+\frac{\omega_{i}}{2}+\frac{\pi}{4}\right): \operatorname{tg}\left(\omega_{l}+\frac{\omega_{i}}{2}+\frac{\pi}{4}\right)=-1,
\end{gathered}
$$

oder:

folglich:

$$
\operatorname{tg}\left(\omega_{k}+\frac{\omega_{i}}{2}+\frac{\pi}{4}\right)=-\operatorname{tg}\left(\omega_{l}+\frac{\omega_{i}}{2}+\frac{\pi}{4}\right),
$$

oder

$$
\omega_{k}+\frac{\omega_{i}}{2}+\frac{\pi}{4}=k \pi-\left(\omega_{l}+\frac{\omega_{i}}{2}+\frac{\pi}{4}\right),
$$

$$
\omega_{i}+\omega_{k}+\omega_{l}=\frac{2 k-1}{2} \pi .
$$

Anmerkung. Sind die Ordinatenwinkel zweier Punkte von $p^{3}$ bekannt, so liefert der vorstehende Satz den Ordinatenwinkel des dritten Schnittpunktes von $p^{3}$ mit der jene zwei Punkte verbindenden Geraden, wodurch, weil Ordinatenwinkel, die sich nur um ein Vielfaches von $\pi$ voneinander unterscheiden, ein und dasselbe Element liefern, der dritte Schnittpunkt eindeutig bestimmt ist. Es müssen also (weil, wenn man zwei Punkte von $p^{3}$ einander immer mehr nähern läßt, bis sie schließlich in einen Punkt hineinfallen, der Tangentialpunkt des letztern Punktes der dritte Schnittpunkt von $p^{3}$ mit der die ersten zwei verbindenden Geraden [Tangente] ist) die Ordinatenwinkel $\omega_{i^{\prime}}$ und $\omega_{i}$ eines Punktes $P_{i}$, und seines Tangentialpunktes $P_{i}$ der Forderung genügen :

$$
2 \omega_{i^{\prime}}+\omega_{i}=\frac{2 k+1}{2} \pi,
$$

was auch direkt (mit Hilfe des ersten Ergebnisses der vorigen Nummer) ähnlich wie vorher bewiesen werden kann. Wenn 
also $P_{i^{\prime}}$ gegeben ist, so ist $P_{i}$ eindeutig bestimmt, denn es ist $\omega_{i}=\frac{\pi}{2}-2 \omega_{i^{\prime}}($ für $k=0)$; ist aber $P_{i}$ gegeben, so ist $P_{i^{\prime}}$ nur zwoideutig bestimmt, denn es ist $\omega_{i^{\prime}}=\frac{\pi}{4}-\frac{\omega_{i}}{2}$ (für $k=0$ ) und $\omega_{i^{\prime}}=\frac{3 \pi}{4}-\frac{\omega_{i}}{2}($ für $k=1)$, was wir auch schon längst wissen.

Soll nunmehr $P_{i}$, mit seinem Tangentialpunkt zusammenfallen, so ist dann und nur dann:

$$
3 \omega_{i^{\prime}}=\frac{2 k+1}{2} \pi
$$

also: $\omega_{i^{\prime}}=\frac{\pi}{6}($ für $k=0), \omega_{i^{\prime}}=\frac{\pi}{2}\left(\right.$ für $k=1$ ) und $\omega_{i^{\prime}}=\frac{5 \pi}{6}$ (für $k=2$ ), welche drei Ordinatenwinkel die bekannten Wendepunkte $P_{c}, P_{a}, P_{b}$ liefern.

65. Mit Hilfe des ersten Ergebnisses in Nr.63 kann nun Folgendes bewiesen werden:

Liegen drei Punkte $P_{i}, P_{k}, P_{l}$ von $p^{3}$, welche von $P$ aus durch $g_{i}, g_{k}, g_{l}$ projiziert werden, in einer Geraden, so liegen auch diejenigen drei Punkte von $p^{3}$, welche von $P$ aus durch die drei in $(P)^{2}$ zu $g_{i}^{(1)}, g_{k}^{(1)}, g_{l}^{(1)}$, den ersten Repräsentanten von $g_{i}, g_{k}, g_{l}$, zugepaarten Strahlen projiziert werden und welche drei Punkte also (Auflösung 3 der Aufgabe 5 in Nr.61) die Tangentialpunkte von $P_{i}, P_{k}, P_{l}$ sind, in einer zweiten Geraden, welche letztere die Begleiterin der erstern Geraden genannt wird.

Beweis. Die auf $p^{2}$ liegenden Pole $G_{k}$ und $G_{l}$ (von $g_{k}$ und $g_{l}$ ), welche mit $Q_{i} \equiv g_{i} p$ in einer Geraden liegen (II), und also auch die ersten Repräsentanten $g_{k}^{(1)} \equiv P G_{k}$ und $g_{l}^{(1)} \equiv P G_{\text {, werden durch }}$ das Strahlenpaar $g_{i} g_{i}^{\prime}$ von $(P)^{2}$ harmonisch getrennt; da in bezug auf $p^{2} P$ der Pol von $p$ und $(P)^{2}$ die Involution konjugierter Strahlen um $P$ sind und also $g_{i}^{\prime}$ die Polare von $Q_{i} \equiv g_{i} p$ in bezug auf $p^{2}$. Bezeichnen wir nun für einen Augenblick die in $(P)^{2}$ zu $g_{k}^{(1)}$ und $g_{l}^{(1)}$ zugepaarten Strahlen mit $g_{x}$ bzw. $g_{y}$, so müssen, weil $g_{i} g_{i}^{\prime}, g_{k}^{(1)} g_{x}, g_{l}^{(1)} g_{y}$ drei Strahlenpaare in einer Involution, nämlich $(P)^{2}$, bilden und also $g_{k}^{(1)} g_{l}^{(1)} g_{i} g_{i}^{\prime} \bar{\wedge} g_{x} g_{y} g_{i}^{\prime} g_{i}$ ist, auch $g_{x}$ und $g_{y}$ durch das Strahlenpaar $g_{i} g_{i}^{\prime}$ harmonisch getrennt sein; mithin bilden $g_{x} g_{y}$ ein Strahlenpaar in der auf $(P)^{2}$ sich 
stützenden Involution, deren Doppelstrahlen aus dem Strahlenpaare $g_{i} g_{i}^{\prime}$ von $(P)^{2}$ bestehen. Folglich müssen (nach der Umkehrung des ersten Ergebnisses in Nr.63) die beiden Punkte von $p^{3}$, welche von $P$ aus durch die in $(P)^{2}$ zu $g_{k}^{(1)}$ und $g_{l}^{(1)}$ zugepaarten Strahlen $g_{x}$ und $g_{y}$ projiziert werden, mit demjenigen Punkte von $p^{3}$ in einer Geraden liegen, welcher von $P$ aus durch den in $(P)^{2}$ zu dem Repräsentanten des Strahlenpaares $g_{i} g_{i}^{\prime}$, also (Nr. 24) zu dem ersten Repräsentanten $g_{i}^{(1)}$ von $g_{i}$ und $g_{i}^{\prime}$, zugepaarten Strahl projiziert wird, was zu beweisen war.

Weil nun die Tangentialpunkte dreier in einer Geraden liegender Punkte $P_{i}, P_{k}, P_{l}$ von $p^{3}$ gleichfalls in einer Geraden liegen, so liegen auch die drei Tangentialpunkte dieser Tangentialpunkte, also die zweiten Tangentialpunkte von $P_{i}, P_{k}, P_{l}$, und mithin auch die dritten, vierten und überhaupt die $n$-ten Tangentialpunkte von $P_{i}, P_{k}, P_{l}$ in je einer Geraden. Nunmehr wird aber der Tangentialpunkt von $P_{i}$ aus $P$ durch den in $(P)^{2} \mathrm{zu}$ $g_{i}^{(1)}$, dem ersten Repräsentanten von $g_{i}$, zugepaarten Strahl projiziert und der Tangentialpunkt dieses Tangentialpunktes, also der zweite Tangentialpunkt von $P_{i}$, wird (weil der den ersten Tangentialpunkt aus $P$ projizierende Strahl denselben ersten Repräsentanten hat, wie der zu ihm in $(P)^{2}$ zugepaarte $g_{i}^{(1)}$, nämlich den zweiten Repräsentanten $g_{i}^{(2)} \equiv g_{i}^{(1)(1)}$ von $g_{i}$ ) von $P$ aus durch den in $(P)^{2}$ zu $g_{i}^{(2)}$ zugepaarten Strahl projiziert, und überhaupt wird der $n$-te Tangentialpunkt von $P_{i}$, wie man leicht in gleicher Weise durch den Schluß von $n$ auf $n+1$ beweisen kann, von $P$ aus durch den in $(P)^{2}$ zu $g_{i}^{(n)}$, dem $n$-ten Repräsentanten von $g_{i}$, zugepaarten Strahl projiziert. Mithin:

Liegen drei Punkte $P_{i}, P_{k}, P_{l}$ von $p^{3}$, welche von $P$ aus durch $g_{i}, g_{k}, g_{l}$ projiziert werden, in einer Geraden, so liegen auch diejenigen drei Punkte von $p^{3}$, welche von $P$ aus durch die drei in $(P)^{2}$ zu $g_{i}^{(n)}, g_{k}^{(n)}, g_{l}^{(n)}$, den $n$-ten Repräsentanten von $g_{i}, g_{k}, g_{l}$, zugepaarten Strahlen projiziert werden und welche drei Punkte also die $n$-ten Tangentialpunkte von $P_{i}, P_{k}, P_{l}$ sind, in einer Geraden, welche letztere die $n$-te Begleiterin der erstern ist.

Dies ergibt sich auch aus dem Satze 27. Sind nämlich $\omega_{i}, \omega_{k}, \omega_{l}, \omega_{i}^{(n)}, \omega_{k}^{(n)}, \omega_{l}^{(n)}$ der Reihe nach die Ordinatenwinkel von $g_{i}, g_{k}, g_{l}, g_{i}^{(n)}, g_{k}^{(n)}, g_{l}^{(n)}$ und mithin $\omega_{i}^{(n)}+\frac{\pi}{2}, \omega_{k}^{(n)}+\frac{\pi}{2}, \omega_{l}^{(n)}+\frac{\pi}{2}$ die 
von den in $(P)^{2}$ zu $g_{i}^{(n)}, g_{k}^{(n)}, g_{l}^{(n)}$ zugepaarten Strahlen, so ist nach Satz 15 (Nr. 26):

$$
\begin{gathered}
\left(\omega_{i}^{(n)}+\frac{\pi}{2}\right)+\left(\omega_{k}^{(n)}+\frac{\pi}{2}\right)+\left(\omega_{l}^{(n)}+\frac{\pi}{2}\right) \\
=(-2)^{n}\left(\omega_{i}+\omega_{k}+\omega_{l}\right)+\frac{3 \pi}{2} .
\end{gathered}
$$

Sollen nun $P_{i}, P_{k}, P_{l}$ in einer Geraden liegen und also nach Satz 27:

so wird auch

$$
\omega_{i}+\omega_{k}+\omega_{l}=\frac{2 k+1}{2},
$$

$$
\begin{aligned}
& \left(\omega_{i}^{(n)}+\frac{\pi}{2}\right)+\left(\omega_{k}^{(n)}+\frac{\pi}{2}\right)+\left(\omega_{i}^{(n)}+\frac{\pi}{2}\right) \\
& =(-2)^{n} \frac{2 k+1}{2} \pi+\frac{3 \pi}{2}=\frac{2 k^{\prime}+1}{2} \pi
\end{aligned}
$$

sein und mithin (nach demselben Satze 27) werden auch die $n$-ten Tangentialpunkte von $P_{i}, P_{k}, P_{l}$ in einer Geraden liegen müssen.

66. Mit Hilfe des ersten Ergebnisses in Nr. 63 kann auch Folgendes bewiesen werden:

Sind $P_{1}, P_{4}, P_{7}$ die Schnittpunkte von $p^{3}$ mit irgendeiner Geraden und $P_{2}, P_{5}, P_{8}$ die mit einer zweiten Geraden, so schneidet $p^{3}$ die drei Geraden $P_{1} P_{2}, P_{4} P_{5}, P_{7} P_{8}$ in drei neuen Punkten $P_{3}, P_{6}, P_{9}$, welche wiederum auf einer Geraden liegen.

Beweis. Es sei, wie bisher, die irgendeinen Punkt $P_{i}$ von $p^{3}$ aus $P$ projizierende Gerade mit $g_{i}$, ihr Pol mit $G_{i}$ und ihr Schnittpunkt mit der Polare $p$ von $P$ mit $Q_{i}$ bezeichnet. Die Pole der vier $P_{1}, P_{2}, P_{4}, P_{5}$ von $P$ aus projizierenden Geraden $g_{1}, g_{2}, g_{4}, g_{5}$ bilden ein $p^{2}$ eingeschriebenes vollständiges Viereck $G_{1} G_{2} G_{4} G_{5}$, von welchem, weil $P_{1}$ und $P_{2}$ mit $P_{3}, P_{4}$ und $P_{5}$ mit $P_{6}, P_{1}$ und $P_{4}$ mit $P_{7}, P_{2}$ und $P_{5}$ mit $P_{8}$ in je einer Geraden liegen, das Paar Gegenseiten $G_{1} G_{2}$ und $G_{4} G_{5}$ durch $Q_{3}$ und $Q_{6}$, die Schnittpunkte von $p$ mit $g_{3}$ und $g_{6}$, und das Paar Gegenseiten $G_{1} G_{4}$ und $G_{2} G_{5}$ durch $Q_{7}$ und $Q_{8}$, die Schnittpunkte von $p$ mit $g_{7}$ und $g_{8}$, gehen (nach II in Nr.55). Es bilden also nach dem bekannten Satze von Desargues die drei Punktepaare, nämlich $Q_{3} Q_{6}, Q_{7} Q_{8}$ und das aus den Doppelpunkten der von $A B C$ erzeugten Involution $(p)^{2}$ bestehende Paar, in denen $p$ zwei Paar 
Gegenseiten des $p^{2}$ eingeschriebenen Vierecks $G_{1} G_{2} G_{4} G_{5}$ und $p^{2}$ (nach Nr.2) schneidet, eine Involution und zwar eine auf $(p)^{2}$ sich stützende. Mithin bilden die diese drei Punktepaare von $P$ aus projizierenden Geraden, nämlich $g_{3} g_{6}, g_{7} g_{8}$ und die beiden Doppelstrahlen der von $A B C$ erzeugten Involution $(P)^{2}$ (nach Nr. 5), drei Strahlenpaare einer auf $(P)^{2}$ sich stützenden Involution. Folglich muß nach dem ersten Ergebnisse in Nr. 63 das Punktepaar $P_{3} P_{6}$ von $p^{3}$ (welches durch $g_{3} g_{6}$ von $P$ aus projiziert wird) mit demjenigen Punkte von $p^{3}$ in einer Geraden liegen, mit welchem auch das Punktepaar $P_{7} P_{8}$ von $p^{3}$ (welches durch $g_{7} g_{8}$ von $P$ aus projiziert wird) in einer Geraden liegt und welcher Punkt also $P_{9}$ ist, was zu beweisen war.

Auch dies ergibt sich gleichfalls aus dem Satze 27. Nach Voraussetzung muß nämlich sein:

$$
\omega_{1}+\omega_{4}+\omega_{7}=\frac{2 k_{1}+1}{2} \pi, \quad \omega_{2}+\omega_{5}+\omega_{8}=\frac{2 k_{2}+1}{2} \pi
$$

und

$$
\begin{aligned}
& \omega_{3}=\frac{2 k_{3}+1}{2} \pi-\omega_{1}-\omega_{2} \\
& \omega_{6}=\frac{2 k_{4}+1}{2} \pi-\omega_{4}-\omega_{5} \\
& \omega_{9}=\frac{2 k_{5}+1}{2} \pi-\omega_{7}-\omega_{8},
\end{aligned}
$$

also ist:

$$
\begin{aligned}
\omega_{3}+\omega_{6}+\omega_{9} & =\frac{2\left(k_{3}+k_{4}+k_{5}\right)+3}{2} \pi-\left(\omega_{1}+\omega_{4}+\omega_{7}\right) \\
-\left(\omega_{2}+\omega_{5}+\omega_{8}\right) & =\frac{2\left(k_{3}+k_{4}+k_{5}\right)+3}{2} \pi-\frac{2 k_{1}+1}{2} \pi \\
-\frac{2 k_{2}+1}{2} \pi & =\frac{2\left(k_{3}+k_{4}+k_{5}-k_{1}-k_{2}\right)+1}{2} \pi=\frac{2 k^{\prime}+1}{2} \pi
\end{aligned}
$$

und folglich müssen auch $P_{3}, P_{6}, P_{9}$ in einer Geraden liegen.

In gleicher Weise kann auch Folgendes bewiesen werden:

Die drei Paar Gegenseiten eines $p^{3}$ eingeschriebenen vollständigen Vierecks $P_{1} P_{2} P_{3} P_{4}$ schneiden $p^{3}$ je zum drittenmal in drei neuen Punktepaaren, deren drei Sehnen durch einen und denselben Punkt von $p^{3}$ laufen.

Sind nämlich $P_{5}, P_{6} ; P_{7}, P_{8} ; P_{9}, P_{10}$ der Reihe nach die dritten Schnittpunkte von $p^{3}$ mit den Viereckseiten $P_{1} P_{2}, P_{3} P_{4}$; $P_{1} P_{3}, P_{2} P_{4} ; P_{1} P_{4}, P_{2} P_{3}$, so müssen die drei Paar Gegenseiten 
$G_{1} G_{2}, G_{3} G_{4} ; G_{1} G_{3}, G_{2} G_{4} ; G_{1} G_{4}, G_{2} G_{3}$ des $p^{2}$ eingeschriebenen vollständigen Vierecks $G_{1} G_{2} G_{3} G_{4}$ der Reihe nach von $p$ in den drei Punktepaaren $Q_{5} Q_{6}, Q_{7} Q_{8}, Q_{9} Q_{10}$ geschnitten werden (nach II in Nr. 55), welche Punktepaare, wie wir sahen, eine auf $(p)^{2}$ sich stützende Involution bilden; diese drei Punktepaare werden nun von $P$ aus durch die drei Strahlenpaare $g_{5} g_{6}, g_{7} g_{8}, g_{9} g_{10}$ projiziert, welche also eine auf $(P)^{2}$ sich stützende Strahleninvolution bilden. Mithin müssen die durch diese drei Strahlenpaare von $P$ aus projizierten Punktepaare $P_{5} P_{6}, P_{7} P_{8}, P_{9} P_{10}$ von $p^{3}$, nach dem ersten Ergebnisse in Nr.63, mit einem und demselben Punkte von $p^{3}$ in je einer Geraden liegen.

\section{Aus II ergibt sich ferner:}

Von den drei Diagonalpunkten eines $p^{3}$ eingeschriebenen vollständigen Vierecks können niemals alle drei zugleich auf $p^{3}$ liegen. Zwei dieser drei Diagonalpunkte liegen dann und nur dann auf $p^{3}$, wenn das vollständige Viereck aus solchen zwei Punktepaaren von $p^{3}$ besteht, welche von $P$ aus durch zwei Strahlenpaare von $(P)^{2}$ projiziert werden; und zwar bestehen alsdann diese zwei Diagonalpunkte (welche diejenigen sein müssen, durch denen nur die je zwei kein solches Punktepaar bildende Ecken verbindenden Seiten des Vierecks gehen) aus einem dritten ebensolchen Punktepaare von $p^{3}$ und das im dritten Diagonalpunkte sich schneidende Paar je eins der zwei Punktepaare verbindender Gegenseiten des Vierecks schneidet $p^{3}$ zum drittenmal in solchen zwei Punkten, welche mit dem gemeinsamen Tangentialpunkte der erstern zwei Diagonalpunkte in einer Geraden liegen.

Beweis. Ist $P_{1} P_{2} P_{3} P_{4}$ das $p^{3}$ eingeschriebene vollständige Viereck und sollten alle drei Diagonalpunkte des Vierecks $p^{3}$ angehören, sollte also jedes Paar Gegenseiten des Vierecks von $p^{3}$ zum drittenmal in einem Punkte geschnitten werden, so müßte nach II jedes Paar Gegenseiten des dem Polarkegelschnitt $p^{2}$ von $P$ eingeschriebenen vollständigen Vierecks $G_{1} G_{2} G_{3} G_{4}$ von $p$, der Polare von $P$, in einem Punkt geschnitten werden und also müßten alle drei Diagonalpunkte von $G_{1} G_{2} G_{3} G_{4}$ auf einer und derselben Geraden, nämlich auf $p$, liegen, was aber unmöglich ist. Ferner gehören (nach II) zwei Diagonalpunkte, die etwa $P_{m}$ und $P_{n}$ heißen mögen, des 
Vierecks $P_{1} P_{2} P_{3} P_{4}$ dann und nur dann $p^{3}$ an, wenn zwei Diagonalpunkte des $p^{2}$ eingeschriebenen Vierecks $G_{1} G_{2} G_{3} G_{4}$ auf $p$ liegen und also die Schnittpunkte $Q_{m}$ und $Q_{n}$ von $p$ mit den $P_{m}$ und $P_{n}$ von $P$ aus projizierenden Geraden $g_{m}$ und $g_{n}$ sind; dies ist aber, weil $p$ die Polare von $P$ auch in bezug auf $p^{2}$ ist (Nr.2), dann und nur dann der Fall, wenn $P$ der dritte Diagonalpunkt von $G_{1} G_{2} G_{3} G_{4}$ ist und also (Satz 1 in Nr. 7) die vier $P_{1}, P_{2}, P_{3}, P_{4}$ von $P$ aus projizierenden Polaren $g_{1}, g_{2}, g_{3}, g_{4}$ (von $G_{1}, G_{2}, G_{3}, G_{4}$ ) aus zwei Strahlenpaaren von $(P)^{2}$ bestehen. Derjenige Diagonalpunkt von $P_{1} P_{2} P_{3} P_{4}$, in dem die je eins der zwei Punktepaare (die durch je ein Strahlenpaar von $(P)^{2}$ projiziert werden) verbindenden Gegenseiten sich schneiden und dessen entsprechender Diagonalpunkt in $G_{1} G_{2} G_{3} G_{4}$ der nicht auf $p$ liegende Punkt $P$ ist, kann nach II $p^{3}$ nicht angehören. Alsdann müssen aber die beiden Diagonalpunkte $Q_{m}$ und $Q_{n}$ des $p^{2}$ eingeschriebenen Vierecks $G_{1} G_{2} G_{3} G_{4}$ einander konjugiert sein in bezug auf $p^{2}$ und mithin, weil die Involution der in bezug auf $p^{2}$ konjugierten Punkte auf $p$ mit $(p)^{2}$ identisch ist, ein Punktepaar in $(p)^{2}$ bilden. Folglich müssen dann, weil $(P)^{2} \mathrm{zu}(p)^{2}$ perspektiv ist (Nr.5), $g_{m}$ und $g_{n}$, welche das Punktepaar $Q_{m}$ und $Q_{n}$ von $(p)^{2}$ und ebenso die zwei $p^{3}$ angehörenden Diagonalpunkte $P_{m}$ und $P_{n}$ von $P_{1} P_{2} P_{3} P_{4}$ aus $P$ projizieren, ein Strahlenpaar in $(P)^{2}$ bilden. Sind dann $Q_{x}$ und $Q_{y}$ die Schnittpunkte von $p$ mit dem in $P$ sich schneidenden Paar Gegenseiten von $G_{1} G_{2} G_{3} G_{4}$, so bilden $Q_{x}$ und $Q_{y}$ ein Paar in der von $G_{1} G_{2} G_{3} G_{4}$ in $p$ eingeschnittenen Involution, deren Doppelpunkte aus dem Punktepaare $Q_{m}$ und $Q_{n}$ von $(p)^{2}$ bestehen und welche Involution sich also auf $(p)^{2}$ stützt, und die dritten Schnittpunkte $P_{x}$ und $P_{y}$ von $p^{3}$ mit demjenigen Paar Gegenseiten von $P_{1} P_{2} P_{3} P_{4}$, welches sich in dem $p^{3}$ nicht angehörenden Diagonalpunkte von $P_{1} P_{2} P_{3} P_{4}$ schneidet, müssen demnach von $P$ aus durch das Strahlenpaar $g_{x} g_{y}$, welches nach II zugleich auch das Punktepaar $Q_{x}$ und $Q_{y}$ projiziert, derjenigen auf $(P)^{2}$ sich stützenden Involution projiziert werden, deren Doppelstrahlen aus dem Paare $g_{m}$ und $g_{n}$ von $(P)^{2}$ bestehen. Folglich müssen (nach der Umkehrung des ersten Ergebnisses in Nr. 63) $P_{x}$ und $P_{y}$ mit demjenigen Punkte von $p^{3}$ in einer Geraden liegen, welcher von $P$ aus durch die in $(P)^{2}$ dem ersten Repräsentanten $g_{m}^{(1)} \equiv g_{n}^{(1)}$, dem Repräsentanten des Strahlenpaares $g_{m} g_{n}$ von $(P)^{2}$, zugepaarten Geraden projiziert wird und 
welcher Punkt also (Auflösung 3 der Aufgabe 5 in Nr.61) der Tangentialpunkt von $P_{m}$ und zugleich von $P_{n}$ ist, was zu beweisen war.

Indem wir die Ecken des vollständigen Vierecks und zwei seiner Diagonalpunkte als die sechs Ecken eines vollständigen Vierseits auffassen, läßt sich das letzte Ergebnis auch wie folgt aussprechen:

Wenn zweimal zwei Gegenpunkte eines vollständigen Vierseits solche Punktepaare von $p^{3}$ sind, die von $P$ aus durch Strahlenpaare von $(P)^{2}$ projiziert werden, so sind es auch die dritten.

68. Mit Hilfe der Aussage III (Nr. 56) ergibt sich:

Verbindet man alle diejenigen Punktepaare von $p^{3}$, welche von $P$, dem isolierten Doppelpunkte von $p^{3}$, aus durch Strahlenpaare von $(P)^{2}$ projiziert werden, mit irgendeinem $p^{3}$ angehörenden Punkte $P_{i}$ durch Strahlenpaare, so bilden diese Strahlenpaare, von denen jedes (wie aus dem Ergebnisse der letzten Nummer hervorgeht) noch durch ein zweites solches Punktepaar von $p^{3}$ geht, eine hyperbolische Involution um $P_{i}$, deren Doppelstrahlen $P_{i} P \equiv g_{i}$ und $P_{i} G_{i}$ sind, wobei $G_{i}$ der Pol von $g_{i}$ in bezug auf $A B C$ und $P_{i} G$ die Tangente $t_{i}$ an $p^{2}$ in $G_{i}$ ist (nach Satz 24 in Nr.53).

Beweis. Bilden $P_{k}$ und $P_{k}^{\prime}$ irgendein solches Punktepaar von $p^{3}$, welches also von $P$ aus durch das Strahlenpaar $g_{k} g_{k}^{\prime}$ von $(P)^{2}$ projiziert wird, und sind $V_{k}$ und $V_{k}^{\prime}$ die zweiten Schnittpunkte von $P_{i} P_{k}$ und $P_{i} P_{k}^{\prime}$ mit dem Polarkegelschnitt $q_{i}^{2}$ von $Q_{i} \equiv g_{i} p$, welcher Polarkegelschnitt durch $P_{i}, P$ und $G_{i}$ geht (Nr.56), so ist der Strahlenwurf $P_{i}\left(P_{k} P_{k}^{\prime} P G_{i}\right)$ zu dem Punktwurf $q_{i}^{2}\left(V_{k} V_{k}^{\prime} P G_{i}\right)$ seiner zweiten Schnittpunkte mit $q_{i}^{2}$ perspektiv, und dieser Punktwurf ist wieder zu dem Strahlenwurf seiner Polaren um $Q_{i}$, nämlich $Q_{i}\left(v_{k} v_{k}^{\prime} p g_{i}\right)$, projektiv (Nr.2). Der letzte Strahlenwurf muß aber ein harmonischer sein, da $v_{k}$ und $v_{k}^{\prime}$ nach III durch die auf $p^{2}$ liegenden Pole $G_{k}$ und $G_{k}^{\prime}$ des Strahlenpaares $g_{k} g_{k}^{\prime}$ und $g_{i}$ durch $P$ gehen und $G_{k}$ und $G_{k}^{\prime}$ in einer durch $P$ gehenden Geraden liegen (Satz 1 in Nr. 7) und durch $P$ und $p$, die Polare von $P$ auch in bezug auf $p^{2}$, harmonisch getrennt sind. Folglich muß auch der erste Strahlenwurf, nämlich $P_{i}\left(P_{k} P_{k}^{\prime} P G_{i}\right)$, ein harmonischer sein. Die $P_{i}$ mit den im vor- 
stehenden Satze erwähnten Punktepaaren von $p^{3}$ verbindenden Strahlenpaare, von denen jedes durch $P_{i} P$ und $P_{i} G_{i}$ harmonisch getrennt ist, müssen also eine hyperbolische Involution um $P_{i}$ bilden, deren Doppelstrahlen $P_{i} P$ und $P_{i} G_{i}$ sind.

69. Mit Hilfe der Aussage III ergibt sich ferner:

Bestimmt man auf jeder durch einen Punkt $P_{i}$ von $p^{3}$ gehenden Geraden $r$, die noch ein Paar weiterer Punkte mit $p^{3}$ gemein hat, den zu $P_{i}$ zugeordneten vierten harmonischen Punkt in bezug auf das Punktepaar, so beschreibt derselbe bei der Drehung von $r$ um $P_{i}$ einen Kegelschnitt, den sogenannten Polarkegelschnitt von $P_{i}$ in bezug auf $p^{3}$. Dieser Kegelschnitt geht durch den Punkt $P_{i}$, den isolierten Doppelpunkt $P$ von $p^{3}$, die Berührungspunkte der beiden von $P_{i}$ an $p^{3}$ gehenden Tangenten und durch den Pol $G_{i}$ der $P_{i}$ von $P$ aus projizierenden Geraden $g_{i}$ (in bezug auf $A B C)$ und hat in $P_{i}$ dieselbe Tangente, wie $p^{3}$, und in $P$ die zu $g_{i}$ in $(H)^{2}$ zugepaarte Gerade zur Tangente.

Beweis. Der von $r$ um den auf $p^{3}$ und $q_{i}^{2}$, dem Polarkegelschnitt von $Q_{i} \equiv p g_{i}$ (Nr.56), liegenden Punkt $P_{i}$ beschriebene Strahlenbüschel $P_{i}(r)$ ist zu der von $V$, dem zweiten Schnittpunkte von $r$ mit $q_{i}^{2}$, beschriebenen Punktreihe $q_{i}^{2}(V)$ perspektiv; diese Punktreihe ist wieder zu dem von $v$, der durch $Q_{i}$ gehenden Polare von $V$ in bezug auf $A B C$, beschriebenen Strahlenbüschel $Q_{i}(v)$ projektiv (nach Nr. 2); $Q_{i}(v)$ ist ferner zu dem von der durch $G_{i}$, den auf dem Polarkegelschnitt $p^{2}$ von $P$ liegenden Pol von $g_{i}$, gehenden, zu $v$ in bezug auf $p^{2}$ konjugierten Geraden $s$ beschriebenen Strahlenbüschel $G_{i}(s)$ projektiv; $G_{i}(s)$ ist weiter zu der von $G_{s}$, dem zweiten Schnittpunkte von $s$ mit $p^{2}$, beschriebenen Punktreihe $p^{2}\left(G_{s}\right)$ perspektiv, und diese endlich ist zu dem von $g_{s}$, der durch $P$ gehenden Polare von $G_{s}$ in bezug auf $A B C$, beschriebenen Strahlenbüschel $P\left(g_{s}\right)$ projektiv. Folglich sind auch der erste und der letzte Strahlenbüschel, nämlich $P_{i}(r)$ und $P\left(g_{s}\right)$ zueinander projektiv. Nunmehr wird aber $p^{2}$ von den beiden in bezug auf ihn konjugierten Geraden $v$ und $s$ in vier harmonischen Punkten geschnitten, von denen die beiden Schnittpunkte $G_{i}$ und $G_{s}$ von $p^{2}$ mit $s$ die Pole der durch $P$ gehenden Geraden $g_{i}$ und $g_{s}$ sind und die beiden Schnittpunkte von $p^{2}$ mit $v$ die Pole der von $P$ aus die beiden weitern Schnitt- 
punkte (außer $P_{i}$ ) von $r$ mit $p^{3}$ projizierenden Geraden sind; mithin wird auch um $P g_{s}$ von $g_{i}$ durch die beiden letztern Geraden harmonisch getrennt und der auf $r$ zu $P_{i}$ zugeordnete vierte harmonische Punkt in bezug auf die beiden weitern Schnittpunkte von $r$ mit $p^{3}$ muß also der Schnittpunkt von $r$ mit $g_{s}$ sein. Der zu $P_{i}$ vierte harmonische Punkt, als Schnittpunkt zweier entsprechender Strahlen in den projektiven Büscheln $P_{i}(r)$ und $P\left(g_{s}\right)$, muß nun einen solchen Kegelschnitt beschreiben, welcher durch die Grundpunkte $P_{i}$ und $P$ der beiden projektiven Büschel geht und (weil, wenn $g_{s}$ nach $P P_{i} \equiv g_{i}$ kommt, $G_{s}$ mit $G_{i}, s\left(\equiv G_{i} G_{s}\right)$ mit der Tangente $t_{i}$ von $p^{2}$ in $G_{i}$, die durch $Q_{i}$ gehende, zu $s$ in bezug auf $p^{2}$ konjugierte Gerade $v$ mit $Q_{i} G_{i}$ und $r$ also nach Satz 26 (Nr.59) mit der Tangente von $p^{3}$ in $P_{i}$ zusammenfallen und mithin dem Strahle $P P_{i}$, als dem Büschel um $P$ angehörig, im Büschel um $P_{i}$ die Tangente von $p^{3}$ in $P_{i}$ entspricht) in $P_{i}$ dieselbe Tangente, wie $p^{3}$, hat und (weil, wenn $r$ nach $P_{i} P$ kommt, $V$ mit $P(N r .56), v$ mit $p, s$ mit $G_{i} P, G_{s}$ mit dem zweiten Schnittpunkte von $p^{2}$ mit $G_{i} P$ und also $g_{s}$ (nach Satz 1 in Nr. 7) mit der zu $g_{i}$ in $(P)^{2}$ zugepaarten Geraden zusammenfallen und mithin die letzte Gerade im Büschel um $P$ dem Strahle $P_{i} P$, als dem Büschel um $P_{i}$ angehörig, entspricht) in $P$ von der zu $g_{i}$ in $(P)^{2}$ zugepaarten Geraden tangiert wird. Dieser Kegelschnitt geht aber auch durch die Berührungspunkte der beiden von $P_{i}$ an $p^{3}$ gehenden Tangenten, weil in einem solchen Berührungspunkte zwei der vier harmonischen Punkte, nämlich die beiden weitern Schnittpunkte (außer $P_{i}$ ) der Tangente mit $p^{3}$, und mithin auch der dritte, nämlich der in bezug auf diese zwei zu $P_{i}$ zugeordnete vierte harmonische Punkt, vereinigt liegen, und außerdem noch durch $G_{i}$, da nach Satz 24 (Nr.53) auf der von $P_{i}$ an $p^{2}$ gehenden Tangente $t_{i}$, deren Berührungspunkt $G_{i}$ ist, $G_{i}$ von $P_{i}$ durch die beiden weitern Schnittpunkte von $t_{i}$ mit $p^{3}$ harmonisch getrennt ist.

Wenn aber $P_{i}$ ein Wendepunkt von $p^{3}$, also (Nr. 60) einer der drei Schnittpunkte $P_{a}, P_{b}, P_{c}$ von $p$ mit den Seiten von $A B C$, etwa $P_{a}$, ist, so ist $g_{i} \equiv P P_{a} \equiv p_{A}^{\prime}, Q_{i} \equiv P_{a}$ und $G_{i} \equiv P_{A}^{\prime}$ liegt auf der Ecktransversale $P A \equiv p_{A}$ (Nr. 60), welche letzte, weil $P_{a} \equiv p p_{A}^{\prime}$ ist, $p_{A} p_{A}^{\prime}$ ein Strahlenpaar in $(P)^{2}$ bilden und in bezug auf $p^{2}$ die Involution konjugierter Strahlen um $P$ mit $(P)^{2}$ identisch ist und $p$ die Polare von $P$ ist (Nr. 2), die Polare von 
$P_{a}$ in bezug auf $p^{2}$ ist. Mithin muß in diesem Falle in der Reihe der obigen Projektivitäten:

$$
P_{i}(r) \pi q_{i}^{2}(V) \pi Q_{i}(v) \pi G_{i}(s) \pi p^{2}\left(G_{s}\right) \pi P\left(g_{s}\right)
$$

die Projektivität zwischen $Q_{i}(v) \equiv P_{a}(v)$ und $G_{\imath}(s) \equiv P_{A}^{\prime}(s)$ eine ausgeartete sein, indem allen durch $Q_{i} \equiv P_{a}$ gehenden Strablen ein einziger durch $G_{i} \equiv P_{A}^{\prime}$ gehender Strahl, nämlich $p_{A}$, entspricht (da $p_{A}$, als Polare von $P_{a}$ in bezug auf $p^{2}$, allen durch $P_{a}$ gehenden Geraden konjugiert ist) mit Ausnahme eines einzigen durch $Q_{i} \equiv P_{a}$ gehenden Strahles, nämlich des $Q_{i} \equiv P_{a}$ mit $G_{i} \equiv P_{A}^{\prime}$ verbindenden Strahles (welcher die Tangente an $p^{2}$ in $P_{A}^{\prime}$ ist (Nr. 60) und also zu allen durch den Berührungspunkt $P_{A}^{\prime} \equiv G_{i}$ gehenden Geraden konjugiert ist in bezug auf $p^{2}$ ), welchem alle durch $G_{i} \equiv P_{A}^{\prime}$ gehenden Strahlen entsprechen. Nunmehr ist aber $A$ der zweite Schnittpunkt des durch $G_{i} \equiv P_{A}^{\prime}$ gehenden Strahles $p_{A}$ mit $p^{2}$ und $p_{A}$ die durch $P$ gehende Polare dieses zweiten Schnittpunktes, ferner ist die $P_{i} \equiv P_{a}$ mit dem Pole von $Q_{i} G_{i} \equiv P_{a} P_{A}^{\prime}$ verbindende Gerade $r$ die Wendetangente von $p^{3}$ in $P_{a}$ (Nr.60); mithin entspricht der gemeinsame Strahl $p_{A} \equiv P_{A}^{\prime} P$ der beiden projektiven Büschel $G_{i}(s) \equiv P_{A}^{\prime}(s)$ und $P\left(g_{s}\right)$ sich selbst und in den beiden konzentrischen, projektiven Büscheln $Q_{i}(v) \equiv P_{a}(v)$ und $P_{i}(r) \equiv P_{a}(r)$ entspricht dem Strahle $P_{a} P_{A}^{\prime}$ des erstern Büschels die Wendetangente von $p^{3}$ in $P_{a}$ im letztern. Folglich ist auch die Projektivität zwischen $P_{i}(r) \equiv P_{a}(r)$ und $P\left(g_{s}\right)$ eine ausgeartete, indem allen durch $P_{a}$ gehenden Strahlen der einzige durch $P$ gehende Strahl $p_{A}$ entspricht mit Ausnahme des einzigen durch $P_{a}$ gehenden Strahles, nämlich der Wendetangente von $p^{3}$ in $P_{\alpha}$, welchem wiederum alle durch $P$ gehenden Strahlen entsprechen. Nunmehr muß der Polarkegelschnitt von $P_{a}$ in bezug auf $p^{3}$, welcher das Erzeugnis der ausgearteten Projektivität zwischen den beiden Büscheln $P_{a}(r)$ und $P\left(g_{s}\right)$ ist und also aus den beiden singulären Strahlen derselben besteht, in das aus der Ecktransversale $p_{A}$ und der Wendetangente von $p^{3}$ in $P_{a}$ bestehende Geradenpaar ausarten.

Mithin:

Der Polarkegelschnitt eines Wendepunktes von $p^{3}$ (in bezug auf $p^{3}$ ) artet in dasjenige Geradenpaar aus, welches aus der Wendetangente im selben Wendepunkte und aus derjenigen durch $P$, den isolierten Doppelpunkt von $p^{3}$, 
gehenden Ecktransversale von $A B C$, welche in $(P)^{2}$ zu der denselben Wendepunkt von $P$ aus projizierenden Geraden zugepaart ist, besteht.

\section{\$10.}

70. Mit Hilfe der Aussage III (Nr.56) läßt sich nun der folgende Satz aufstellen, aus dem alle Beziehungen zwischen der Kurve $p^{3}$ und einem durch irgend zwei ihrer Punkte gehenden Kegelschnitte hervorgehen.

Satz 28. Sind $P_{i}$ und $P_{k}$ irgend zwei voneinander verschiedene Punkte von $p^{3}, Q_{i}$ und $Q_{k}$ die Schnittpunkte von $p$, der Polare von $P$, mit den $P_{i}$ und $P_{k}$ von $P$ aus projizierenden Geraden $g_{i}$ und $g_{k}, r$ eine durch $P_{i}$ gehende Gerade und $s$ eine durch $P_{k}, v$ die durch $Q_{i}$ gehende Polare des zweiten Schnittpunktes $V$ von $r$ mit $q_{i}^{2}$, dem durch $P_{i}$ gehenden Polarkegelschnitt von $Q_{i}$, und $w$ die durch $Q_{k}$ gehende Polare des zweiten Schnittpunktes $W$ von $s$ mit $q_{k}^{2}$, dem durch $P_{k}$ gehenden Polarkegelschnitt von $Q_{k}$, und drehen sich $r$ um $Y_{i}$ und $s$ um $P_{k}$ in der Weise, daß die von ihnen beschriebenen Strahlenbüschel $P_{i}(r)$ und $P_{k}(s)$ projektiv sind und also einen Kegelschnitt $k^{2}$ erzeugen, so beschreiben auch $v$ und $w$ zwei projektive Strahlenbüschel $Q_{i}(v)$
Sind $p_{i}$ und $p_{k}$ irgend zwei voneinander verschiedene Strahlen von $P^{3}$, $g_{i}$ und $g_{k}$ die Verbindungsgeraden von $P$, dem Pole von $p$, mit den Schnittpunkten $Q_{i}$ und $Q_{k}$ von $p$ mit $p_{i}$ und $p_{k}$, also $g_{i} \equiv P Q_{i}$ und $g_{k} \equiv P Q_{k}, M$ ein auf $p_{i}$ liegender Punkt und $N$ ein auf $p_{k}, L$ der auf $g_{i}$ liegende Pol der zweiten von $M$ an $G_{i}^{2}$, den $p_{i}$ berührenden Polarkegelschnitt von $g_{i}$, gehenden Tangente $l$ und $U$ der auf $g_{k}$ liegende $\mathrm{Pol}$ der zweiten von $N$ an $G_{k}^{2}$, den $p_{k}$ berührenden Polarkegelschnitt von $g_{k}$, gehenden Tangente $u$, und bewegen $\operatorname{sich} M$ auf $p_{i}$ und $N$ auf $p_{k}$ derart, daß die von ihnen beschriebenen Punktreihen $p_{i}(M)$ und $p_{k}(N)$ projektiv sind und also einen Kegelschnitt $C^{2}$ erzeugen, so beschreiben auch $L$ und $U$ zwei projektive Punktreihen $g_{i}(L)$ und $g_{k}(U)$, deren Erzeugnis ein 
und $Q_{k}(w)$, deren Erzeugnis ein zweiter Kegelschnitt $x^{2}$ ist, welchen zweiten wir den aus dem ersten Kegelschnitt a bgeleiteten nennen wollen. Der durch $P_{i}(r)$ und $P_{k}(s)$ erzeugte Kegelschnitt $k^{2}$ hat nun mit $p^{3}$ außer $P_{i}$ und $P_{k}$ diejenigen vier Punkte $P_{l}, P_{m}, P_{n}$ und $P_{o}$ und nur diejenigen vier gemein, welche von $P$ aus durch $g_{l}, g_{m}, g_{n}$ und $g_{o}$, die Polaren der vier Schnittpunkte $G_{l}, G_{m}, G_{n}, G_{o}$ des Polarkegelschnitts $p^{2}$ von $P$ mit dem abgeleiteten, durch $Q_{i}(v)$ und $Q_{k}(w)$ erzeugten Kegelschnitt $x^{2}$, projiziert werden. zweiter Kegelschnitt $\Gamma^{2}$ ist, welchen zweiten wir den aus dem ersten Kegelschnitt ab geleiteten nennen wollen. Der durch $p_{i}(M)$ und $p_{k}(N)$ erzeugte Kegelschnitt $C^{2}$ hat nun mit $P^{3}$ außer $p_{i}$ und $p_{k}$ diejenigen vier Strahlen $p_{l}$, $p_{m}, p_{n}$ und $p_{o}$ und nur diejenigen vier gemein, welche von $p$ in den vier Punkten $Q_{l}, Q_{m}, Q_{n}$ und $Q_{o}$, den Polen der vier gemeinschaftlichen Tangenten $q_{l}, q_{m}, q_{n}$, $q_{0}$ des Polarkegelschnitts $P^{2}$ von $p$ und des abgeleiteten, durch $g_{i}(L)$ und $g_{k}(U)$ erzeugten Kegelschnitts $\Gamma^{2}$, geschnitten werden.

Beweis. Weil $P_{i}(r)$ zu $q_{i}^{2}(V)$ und $P_{k}(s)$ zu $q_{k}^{2}(W)$ perspektiv und $q_{i}^{2}(V) \pi Q_{i}(v)$ und $q_{k}^{2}(W) \pi Q_{k}(w)$ und mithin auch $P_{i}(r)$ $\pi Q_{i}(v)$ und $P_{k}(s) \pi Q_{k}(w)$ sind, so muß $Q_{i}(v) \pi Q_{k}(w)$ sein, wenn $P_{i}(r) \pi P_{k}(s)$ ist. Wenn nun der durch $P_{i}(r)$ und $P_{k}(s)$ erzeugte Kegelschnitt mit $p^{3}$ außer $P_{i}$ und $P_{k}$ noch irgendeinen Punkt, etwa $P_{m}$, gemein hat, wenn also zwei entsprechende Strahlen $r$ und $s$ von $P_{i}$ und $P_{k}$ durch $P_{m}$ gehen, so müssen die in den Büscheln um $Q_{i}$ und $Q_{k}$ einander entsprechenden Strahlen $v$ und $w$, die Polaren der zweiten Schnittpunkte von $r$ mit $q_{i}^{2}$ und von $s$ mit $q_{k}^{2}$, nach III durch $G_{m}$, den auf $p^{2}$ liegenden Pol der $P_{m}$ von $P$ aus projizierenden Geraden $g_{m}$, gehen und also muß dann der abgeleitete Kegelschnitt, nämlich der durch $Q_{i}(v)$ und $Q_{k}(w)$ erzeugte, mit $p^{2}$ den Punkt $G_{m}$ gemein haben; und umgekehrt. Hiermit ist der vorstehende Satz bewiesen.

Der durch $P_{i}(r)$ und $P_{k}(s)$ erzeugte Kegelschnitt wird dann und nur dann von $p^{3}$ in einem der beiden Grundpunkte der Büschel, etwa in $\mathrm{P}_{i}$, berührt, wenn dem Strahle $P_{k} P_{i}$, als dem Büschel $P_{k}(s)$ angehörig, im Büschel $P_{i}(r)$ die Tangente von $p^{3}$ in 
$P_{i}$ entspricht; dies ist aber, weil die durch $Q_{i}$ gehende Polare des zweiten Schnittpunktes von $q_{i}^{2}$ mit der Tangente an $p^{3}$ in $P_{i}$ nach Satz 26 (Nr. 59) durch $G_{i}$, den auf $p^{2}$ liegenden Pol von $g_{i}$, gehen muß, dann und nur dann der Fall, wenn in den projektiven Büscheln $Q_{i}(v)$ und $Q_{k}(w)$ die beiden Strahlen $Q_{i} G_{i}$ und $Q_{k} G_{i}$ einander entsprechen und $G_{i}$ also einer der vier Schnittpunkte von $p^{2}$ mit dem abgeleiteten Kegelschnitt, dem durch $Q_{i}(v)$ und $Q_{k}(w)$ erzeugten, ist. Wird nun der abgeleitete Kegelschnitt von $p^{2}$ in $G_{i} n$-punktig berührt, wo $2 \leqq n \leqq 4$ ist, liegen also $n$ der vier gemeinsamen Punkte $G_{l}, G_{m}, G_{n}, G_{o}$ des abgeleiteten Kegelschnitts und $p^{2}$ im Punkte $G_{i}$ unendlich benachbart, so müssen dann $n$ der vier weitern (außer $P_{i}, P_{k}$ ) gemeinsamen Punkte $P_{l}, P_{m}, P_{n}, P_{o}$ des erstern (ursprünglichen) Kegelschnitts, des durch $P_{i}(r)$ und $P_{k}(s)$ erzeugten, und $p^{3}$ im Punkte $P_{i}$ unendlich benachbart liegen und also wird dann der erstere Kegelschnitt von $p^{3}$ in $P_{i}(n+1)$-punktig berührt; und umgekehrt. Wird aber der abgeleitete Kegelschnitt von $p^{2}$ in einem von $G_{i}$ und $G_{k}$ verschiedenen Punkte $G_{l} n$-punktig berührt, so müssen dann $n$ der vier weitern (außer $P_{i}, P_{k}$ ) gemeinsamen Punkte des erstern (ursprünglichen) Kegelschnitts und $p^{3}$ in dem von $P_{i}$ und $P_{k}$ verschiedenen Punkte $P_{l}$ unendlich benachbart liegen und also wird dann der erstere Kegelschnitt von $p^{3}$ in $t_{l}$ nur $n$-punktig berührt; und umgekehrt. Mithin haben wir:

Satz 29. Sind $P_{i}$ und $P_{k}$ zwei beliebige voneinander verschiedene Punkte von $p^{3}, G_{i}$ und $G_{k}$ die auf $p^{2}$, dem Polarkegelschnitt von $P$, liegenden Pole der $P_{i}$ und $P_{k}$ von $P$ aus projizierenden Geraden $g_{i}$ und $g_{k}$, $k^{2}$ irgendein durch $P_{i}$ und $P_{k}$ gehender Kegelschnitt (welcher als Erzeugnis zweier projektiver Büschel $P_{i}(r)$ und $P_{k}(s)$ gedacht werden kann) und $x^{2}$ der aus diesem abgeleitete Kegelschnitt
Sind $p_{i}$ und $p_{k}$ zwei beliebige voneinander verschiedene Strahlen von $P^{3}$, $q_{i}$ und $q_{k}$ die $P^{2}$, den Polarkegelschnitt von $p$, berührenden Polaren der Schnittpunkte $Q_{i}$ und $Q_{k}$ von $p$ mit $p_{i}$ und $p_{k}, C^{2}$ irgendein $p_{i}$ und $p_{k}$ berührender Kegelschnitt (welcher als Erzeugnis zweier projektiver Punktreihen $p_{i}(M)$ und $p_{k}(N)$ gedacht werden kann) und $\Gamma^{2}$ der aus diesem abgeleitete Kegelschnitt, so 
(Satz 28), so gibt es unter den vier weitern (außer $P_{i}$ und $P_{k}$ ) Schnittpunkten von $k^{2}$ mit $p^{3}$ ebensoviel reelle und ebensoviel imaginäre, wieviel es deren unter den vier Schnittpunkten von $x^{2}$ mit $p^{2}$ gibt. Ferner wird $k^{2}$ von $p^{3}$ in $P_{i}$ oder in $P_{k}$ dann und nur dann $(n+1)$ punktig berührt, wo $1 \leqq n$ $\leqq 4$ ist, wenn $x^{2}$ von $p^{2}$ in $G_{i}$ bzw. in $G_{k} n$-punktig berührt wird; in einem von $P_{i}$ und $P_{k}$ verschiedenen Punkte $P_{l}$ wird aber $k^{2}$ von $p^{3}$ dann und nur dann $n$-punktig berührt, wenn $x^{2}$ von $p^{2}$ in $G_{l}$, dem Pole der $P_{l}$ von $P$ aus projizierenden Geraden $g_{l}$, gleichfalls $n$ punktig berührt wird. gibt es unter den vier weitern (außer $p_{i}$ und $p_{k}$ ) gemeinschaftlichen Strahlen (Tangenten) von $C^{2}$ und $P^{3}$ ebensoviel reelle und ebensoviel imaginäre, wieviel es deren unter den vier gemeinschaftlichen Tangenten von $\Gamma^{2}$ und $P^{2}$ gibt. Ferner haben $C^{2}$ und $P^{3}$ in $p_{i}$ oder in $p_{k}$ dann und nur dann einen $(n+1)$-punktigen Berührungspunkt, also $n+1$ aufeinanderfolgende Strablen (Tangenten) gemein (wo $1 \leqq n \leqq 4$ ist), wenn $\Gamma^{2}$ und $P^{2}$ in $q_{i}$ bzw. in $q_{k} n$ aufeinanderfolgende Tangenten gemein haben; in einem von $p_{i}$ und $p_{k}$ verschiedenen Strahle $p_{l}$ aber hat $P^{3}$ mit $C^{2}$ dann und nur dann $n$ aufeinanderfolgende Strahlen gemein, wenn $P^{2}$ mit $\Gamma^{2}$ in $q_{l}$, der Polare des Schnittpunktes $Q_{l}$ von $p$ mit $p_{l}$, gleichfalls $n$ aufeinanderfolgende Tangenten gemein hat.

71. Aus dem Satze 28 folgt ferner:

Satz 30. Sechs Punkte von $p^{3}$ liegen dann und nur dann auf einem Kegelschnitt, wenn irgend zwei und mithin je zwei derjenigen sechs Geraden, welche die sechs Punkte von $P$ aus projizieren, durch ein Punktepaar derjenigen Involution gehen,
Sechs Strahlen von $P^{3}$ tangieren dann und nur dann einen und denselben Kegelschnitt, wenn irgend zwei und infolgedessen je zwei derjenigen sechs Punkte, in denen die sechs Strahlen von $p$ geschnitten werden, von $P$, dem Pole von $p$, aus 
welche auf $p$, der Polare von $P$, durch das aus den Polen (in bezug auf $A B C$ ) der je vier übrigen von jenen sechs Geraden gebildete vollständige Viereck festgelegt wird. durch ein Strahlenpaar derjenigen Involution projiziert werden, welche um $P$ durch das aus den Polaren (in bezug auf $A B C$ ) der je vier übrigen jener sechs Punkte gebildete vollständige Vierseit festgelegt wird.

Beweis. Sind $P_{1}, P_{2}, P_{3}, P_{4}, P_{5}, P_{6}$ sechs Punkte von $p^{3}$, $g_{1}, g_{2}, g_{3}, g_{4}, g_{5}, g_{6}$ die sie von $P$ aus projizierenden Geraden, $G_{1}$, $G_{2}, G_{3}, G_{4}, G_{5}, G_{6}$ und $Q_{1}, Q_{2}, Q_{3}, Q_{4}, Q_{5}, Q_{6}$ deren sechs Pole bzw. deren sechs Schnittpunkte mit $p$, so liegen $P_{1}, P_{2}, P_{3}, P_{4}$, $P_{5}, P_{6}$ dann und nur dann auf einem Kegelschnitt, welcher, wenn etwa $P_{5}$ und $P_{6}$ voneinander verschieden sind, als das Erzeugnis der projektiven Büschel $P_{5}\left(P_{1} P_{2} P_{3} \ldots\right) \pi P_{6}\left(P_{1} P_{2} P_{3} \ldots\right)$ gedacht werden kann, wenn der aus diesem abgeleitete, durch die beiden projektiven Büschel $Q_{5}\left(G_{1} G_{2} G_{3} \ldots\right) \pi Q_{6}\left(G_{1} G_{2} G_{3} \ldots\right)$ erzeugte Kegelschnitt auch durch $G_{4}$ geht (nach Satz 28), wenn also $Q_{5}, Q_{6}$, $G_{1}, G_{2}, G_{3}, G_{4}$ sechs Punkte eines Kegelschnitts sind; dies ist aber nach dem bekannten Satze von Desargues über den einem Viereck umschriebenen Kegelschnitt dann und nur dann der Fall, wenn $Q_{5}$ und $Q_{6}$ ein Punktepaar in der auf $p$ durch das Viereck $G_{1} G_{2} G_{3} G_{4}$ festgelegten Involution bilden; wodurch der vorstehende Satz bewiesen ist.

Anmerkung. Weil das im vorstehenden Satze genannte Viereck dem Polarkegelschnitt $p^{2}$ von $P$ eingeschrieben ist und $p^{2}$ von $p$ in den konjugiert-imaginären Doppelpunkten von $(p)^{2}$ geschnitten wird, so ist die auf $p$ durch jenes Viereck festgelegte Involution eine auf $(p)^{2}$ sich stützende hyperbolische und ist also schon durch ein von den Doppelpunkten von $(p)^{2}$ verschiedenes Punktepaar vollständig bestimmt.

Hieraus ergibt sich nun die folgende lineare Relation zwischen den Ordinatenwinkeln (Nr.64) von sechs auf einem Kegelschnitt liegenden Punkten von $p^{3}$.

Satz 31. Sechs Punkte $P_{1}, P_{2}, P_{3}, P_{4}, P_{5}, P_{6}$ von $p^{3}$ liegen dann und nur dann auf einem Kegelschnitt, wenn ihre Ordinatenwinkel der Forderung genügen:

$$
\omega_{1}+\omega_{2}+\omega_{3}+\omega_{4}+\omega_{5}+\omega_{6}=k \pi,
$$

wo $k$ eine beliebige ganze Zahl bedeutet. 
Beweis. Bezeichnen wir mit $Q_{m}$ und $Q_{n}$ die Schnittpunkte von $p$ mit $G_{1} G_{2}$ und $G_{3} G_{4}$, so liegen nach Satz $30 P_{1}, P_{2}, P_{3}$, $P_{4}, P_{5}, P_{6}$ dann und nur dann auf einem Kegelschnitt, wenn $g_{5}$ und $g_{6}$ durch ein Punktepaar der auf $p$ durch $G_{1} G_{2} G_{3} G_{4}$ festgelegten Involution $(i)^{2}$ gehen, welche Involution, wie wir sahen, sich auf $(p)^{2}$ stützt und in welcher $Q_{m}$ und $Q_{n}$ ein Punktepaar bilden. Nunmehr wird aber die auf $(p)^{2}$ sich stützende Involution $(i)^{2}$ von $P$ aus durch eine auf $(P)^{2}$ sich stützende Strahleninvolution $(I)^{2}$ projiziert. In dieser Strahleninvolution $(I)^{2}$ bilden nun $g_{m}$ und $g_{n}$, die das Punktepaar $Q_{m} Q_{n}$ und zugleich (nach II in Nr. 55 ) die dritten Schnittpunkte $P_{m}$ und $P_{n}$ von $p^{3}$ mit $P_{1} P_{2}$ und $P_{3} P_{4}$ von $P$ aus projizierenden Strahlen, ein Paar; und wenn $g_{5}$ und $g_{6}$ durch ein Punktepaar von $(i)^{2}$ gehen sollen, so werden dann und nur dann auch $g_{5} g_{6}$ ein Strahlenpaar in $(I)^{2}$ bilden müssen. Mithin werden dann und nur dann (nach dem ersten Ergebnisse in Nr.63) die beiden Punktepaare $P_{m} P_{n}$ und $P_{5} P_{6}$, welche von $P$ aus durch zwei Strahlenpaare einer auf $(P)^{2}$ sich stützenden Involution projiziert werden, mit einem und demselben Punkte von $p^{3}$, der etwa $P_{x}$ heißen mag, in je einer Geraden liegen. Dies ist aber nach Satz 27 (Nr.64) nur dann der Fall, wenn

$\omega_{m}+\omega_{n}+\omega_{x}=\frac{2 k_{1}+1}{2} \pi$ und $\omega_{5}+\omega_{6}+\omega_{x}=\frac{2 k_{2}+1}{2} \pi$, wenn also

$$
\omega_{5}+\omega_{6}-\omega_{m}-\omega_{n}=\left(k_{2}-k_{1}\right) \pi .
$$

Weil aber $P_{m}$ und $P_{n}$ die dritten Schnittpunkte von $p^{3}$ mit $P_{1} P_{2}$ und $P_{3} P_{4}$ sind, so ist nach demselben Satze 27:

$$
\omega_{m}=\frac{2 k^{\prime}+1}{2} \pi-\omega_{1}-\omega_{2} \text { und } \omega_{n}=\frac{2 k^{\prime \prime}+1}{2} \pi-\omega_{3}-\omega_{4} .
$$

Substituieren wir diese Werte von $\omega_{m}$ und $\omega_{n}$ in 1), so ergibt sich: $\omega_{5}+\omega_{6}+\omega_{1}+\omega_{2}-\frac{2 k^{\prime}+1}{2} \pi+\omega_{3}+\omega_{4}-\frac{2 k^{\prime \prime}+1}{2} \pi=\left(k_{2}-k_{1}\right) \pi$ oder

$\omega_{1}+\omega_{2}+\omega_{3}+\omega_{4}+\omega_{5}+\omega_{6}=\left(k_{2}+k^{\prime}+k^{\prime \prime}+1-k_{1}\right) \pi=k \pi$ als notwendige und hinreichende Bedingung dafür, daß. $P_{1}, P_{2}$, $P_{3}, P_{4}, P_{5}, P_{6}$ auf einem Kegelschnitt liegen.

72. Der Satz 30 bleibt auch dann noch richtig, wenn von den sechs Punkten von $p^{3} n$ (wo $2 \leqq n \leqq 6$ ) einander unendlich 
benachbart sind, wenn also der Kegelschnitt von $p^{3} n$-punktig berührt werden soll; nur muß alsdann die den $n$-punktigen Berührungspunkt von $P$ aus projizierende Gerade und ebenso ihr Pol $n$-fach gezählt werden, wenn also die $n$-fache Gerade als eine der zwei im Satze 30 erwähnten Geraden oder als diese zwei genommen wird, so liefert ihr Pol $n-1$ bzw. $n-2$ Ecken des im Satze 30 genannten Vierecks, sonst liefert ihr Pol $n$ dieser Ecken. Hierbei muß Folgendes beachtet werden: wird eine solche $n$-fache Gerade als die zwei im Satz 30 erwähnten Geraden genommen, so muß ihr Schnittpunkt mit $p$ ein Doppelpunkt der im selben Satze genannten Involution sein; liefert ferner ein solcher $n$-facher Pol zwei Ecken des Vierecks, so tritt an Stelle der diese zwei Ecken verbindenden Seite des Vierecks die Tangente von $p^{2}$ in dem $n$-fachen Pole; liefert er aber drei Ecken des Vierecks, so treten die Tangente von $p^{2}$ in ihm und die ihn mit der vierten Ecke des Vierecks verbindende Gerade an Stelle eines Paares von Gegenseiten des Vierecks; liefert er endlich alle vier Ecken des Vierecks, so tritt die Tangente von $p^{2}$ in ihm an Stelle eines Paares von Gegenseiten des Vierecks, und die auf $p$ durch das Viereck festgelegte Involution ist (nach Anmerkung in Nr. 71) in den letzteren beiden Fällen durch das einzige Punktepaar bzw. durch den einzigen Doppelpunkt vollkommen bestimmt.

Beweis. Jede Gerade wird bekanntlich von den sämtlichen Kegelschnitten (eines Büschels), welche durch zwei feste Punkte gehen und in einem dritten sich berühren, oder welche durch einen festen Punkt gehen und in einem zweiten sich dreipunktig berühren, oder endlich welche in einem festen Punkte sich vierpunktig berühren, in den Punktepaaren einer solchen Involution geschnitten, in der die Schnittpunkte der Geraden mit der gemeinsamen die beiden erstern festen Punkte verbindenden Sehne und der gemeinsamen Tangente im dritten Punkte ein Punktepaar bilden, bzw. die Schnittpunkte der Geraden mit der gemeinsamen die zwei festen Punkte verbindenden Sehne und der gemeinsamen Tangente im zweiten Punkte, bzw. der Schnittpunkt der Geraden mit der gemeinsamen Tangente im festen Punkte den einen Doppelpunkt bildet. Mithin liegen sechs Punkte $P_{1}, P_{2}, P_{3}, P_{4}, P_{5}, P_{6}$ von $p^{3}$, wenn auch mehrere, ja sogar fünf dieser sechs Punkte einander unendlich benachbart sind, wenn nur zwei der sechs Punkte, etwa $P_{5}$ und $P_{6}$, voneinander verschieden sind, dann und 
und nur dann (nach Satz 29) auf einem Kegelschnitt, wenn $Q_{5}$ und $Q_{6}$ ein Punktepaar in der auf $p$ durch das Viereck $G_{1} G_{2} G_{3} G_{4}$ (das sich auch auf einen einzigen Punkt reduzieren kann) festgelegten, auf $(p)^{2}$ sich stützenden hyperbolischen Involution bilden; und umgekehrt. Hält man nun das Viereck $G_{1} G_{2} G_{3} G_{4}$ (das sich auch auf einen einzigen Punkt reduzieren kann) fest und läßt $Q_{5}$ und $Q_{6}$ in der Weise einander immer näher rücken, daß sie bei dieser Bewegung fortwährend ein Punktepaar in der auf $p$ durch $G_{1} G_{2} G_{3} G_{4}$ festgelegten Involution bildet (was wegen des hyperbolischen Charakters dieser Involution immer möglich ist), so müssen nach dem soeben Bewiesenen auch $P_{5}$ und $P_{6}$ in der Weise auf $p^{3}$ einander immer näher rücken, daß während dieser Bewegung durch die vier festen Punkte $P_{1}, P_{2}, P_{3}, P_{4}$ und die beiden beweglichen $P_{5}$ und $P_{6}$ immer ein Kegelschnitt gelegt werden kann; und umgekehrt. Gehen wir nun zur Grenze über, wo $Q_{5}$ und $Q_{6}$ in einen Doppelpunkt der auf $p$ durch $G_{1} G_{2} G_{3} G_{4}$ festgelegten Involution hineinfallen, so müssen dann $P_{5}$ und $P_{6}$ in einen solchen Punkt von $p^{3}$ hineinfallen, in welchem der durch ihn und $P_{1}, P_{2}, P_{3}, P_{4}$ gelegte Kegelschnitt von $p^{3}$ berührt wird (wo unter Umständen dieser Punkt derjenige ist, in welchem einer oder mehrere der vier Punkte $P_{1}, P_{2}, P_{3}, P_{4}$ liegen); und umgekehrt. Wir sehen also, daß die den Berührungspunkt von $p^{3}$ mit dem Kegelschnitt von $P$ aus projizierende Gerade durch den Doppelpunkt der entsprechenden Involution auf $p$ geht; wodurch unsere Behauptung, daß der Satz 30 immer, sogar wenn alle sechs Punkte einander unendlich benachbart sind, seine Gültigkeit behält, vollständig nachgewiesen ist.

Auch der Satz 31 behält seine Gültigkeit, wenn mehrere der sechs Punkte einander unendlich benachbart sind; es gilt nämlich der

Satz 32. Durch $i(1 \leqq i \leqq 6)$ Punkte $P_{1}, P_{2}, \ldots P_{i}$ von $p^{3}$ geht dann und nur dann ein solcher Kegelschnitt, welcher von $p^{3}$ in $P_{1} m_{1}$-punktig, in $P_{2} m_{2}$-punktig, ... in $P_{i} m_{i}$-punktig berührt wird, wo $m_{1}+m_{2}+\cdots+m_{i}=6$, wenn die Ordinatenwinkel von $P_{1}, P_{2}, \ldots P_{i}$ der Forderung genügen:

$$
m_{1} \omega_{1}+m_{2} \omega_{2}+\cdots+m_{i} \omega_{i}=k \pi,
$$

wo $k$ eine beliebige ganze Zahl bedeutet. 
Dieser Satz kann nach dem eben Bewiesenen direkt analog wie der Satz 31 bewiesen werden; er ergibt sich aber auch aus dem letzten (mit Hilfe von Grenzverfahren). Denn nach diesem Satze 31 geht dann und nur dann durch $P_{1}, P_{2}, P_{3}, P_{4}, P_{5}$ ein $p^{3}$ in $P_{1}$ berührender Kegelschnitt, wenn $2 \omega_{1}+\omega_{2}+\omega_{3}+\omega_{4}+\omega_{5}$ $=k \pi$. Nunmehr geht dann und nur dann durch $P_{1}, P_{2}, P_{3}, P_{4}$ ein $p^{3}$ in $P_{1}$ dreipunktig berührender Kegelschnitt, wenn $3 \omega_{1}+\omega_{2}$ $+\omega_{3}+\omega_{4}=k \pi$. Fahren wir so fort, so ergibt sich: durch $P_{1}$, $P_{2}, \ldots P_{6-m_{1}}$ geht dann und nur dann ein $p^{3}$ in $P_{1} m_{1}$-punktig berührender Kegelschnitt, wenn $m_{1} \omega_{1}+\omega_{2}+\cdots+\omega_{6-m_{1}}=k \pi$. Nun geht durch $P_{1}, P_{2}, \ldots P_{6-m_{1}-1}$ dann und nur dann ein $p^{3}$ in $P_{1} \quad m_{1}$-punktig und in $P_{2}$ einfach berührender Kegelschnitt, wenn $m_{1} \omega_{1}+2 \omega_{2}+\omega_{3}+\cdots+\omega_{6-m_{1}-1}=k \pi$. In dieser Weise fortfahrend gelangt man schließlich zu der im Satze 32 angegebenen Bedingung.

73. Aus dem Satze 30 ergibt sich nun der bekannte Satz über die Kurven dritter Ordnung, nämlich:

Sechs Punkte $P_{1}, P_{2}, P_{3}, P_{4}, P_{5}, P_{6}$ von $p^{3}$ liegen dann und nur dann auf einem Kegelschnitt, wenn irgend drei der die sechs Punkte zu je zwei verbindenden Sehnen, wie etwa $P_{1} P_{2}, P_{3} P_{4}, P_{5} P_{6}$, von $p^{3}$ zum drittenmal in drei Punkten einer Geraden geschnitten werden.

Sind nämlich $P_{x}$ und $P_{y}$ die dritten Schnittpunkte von $p^{3}$ mit $P_{1} P_{2}$ und $P_{3} P_{4}$ und gehen also $G_{1} G_{2}$ und $G_{3} G_{4}$ durch $Q_{x} \equiv p g_{x}$ bzw. $Q_{y} \equiv p g_{y}$ (nach II in Nr. 55), so sind dann und nur dann (nach Satz 30) $Q_{x} Q_{y}$ und $Q_{5} Q_{6}$ zwei Punktepaare einer auf $(p)^{2}$ sich stützenden Involution $(i)^{2}$ und mithin $g_{x} g_{y}$ und $g_{5} g_{6}$ zwei Strahlenpaare in der $(i)^{2}$ von $P$ aus projizierenden, auf $(P)^{2}$ sich stützenden Involution $(I)^{2}$, wenn $P_{1}, P_{2}, P_{3}, P_{4}, P_{5}, P_{6}$ auf einem Kegelschnitt liegen. Folglich (nach dem ersten Ergebnisse in Nr. 63) gehen dann und nur dann $P_{x} P_{y}$ und $P_{5} P_{6}$ durch einen und denselben Punkt von $p^{3}$, welcher letzte Punkt $P_{z}$ heißen möge, oder, was dasselbe aussagt, es liegen dann und nur dann $P_{x}$ und $P_{y}$, die dritten Schnittpunkte von $p^{3}$ mit $P_{1} P_{2}$ und $P_{3} P_{4}$, mit $P_{z}$, dem dritten Schnittpunkt von $p^{3}$ mit $P_{5} P_{6}$, in einer Geraden.

Dasselbe ergibt sich auch aus den Sätzen 27 und 31. Nach Voraussetzung ist: 


$$
\begin{array}{r}
\omega_{x}+\omega_{y}+\omega_{z}=\frac{2 k_{1}+1}{2} \pi-\omega_{1}-\omega_{2} \\
+\frac{2 k_{2}+1}{2} \pi-\omega_{3}-\omega_{4} \\
+\frac{2 k_{3}+1}{2} \pi-\omega_{5}-\omega_{6} \\
=\left(k_{1}+k_{2}+k_{3}\right) \pi+\frac{3 \pi}{2}-\left(\omega_{1}+\omega_{2}+\omega_{3}+\omega_{4}+\omega_{5}+\omega_{6}\right)
\end{array}
$$

und dies ist (nach Satz 31) dann und nur dann gleich

$$
\left(k_{1}+k_{2}+k_{3}\right) \pi+\frac{3 \pi}{2}-k \pi=\frac{2 k^{\prime}+1}{2} \pi,
$$

wenn $P_{1}, P_{2}, P_{3}, P_{4}, P_{5}, P_{6}$ auf einem Kegelschnitt liegen.

Hieraus ergibt sich der zweite bekannte Satz:

Jeder durch vier Punkte $P_{1}, P_{2}, P_{3}, P_{4}$ von $p^{3}$ gehende Kegelschnitt schneidet $p^{3}$ in zwei weitern Punkten, welche mit einem festen Punkte von $p^{3}$, mit dem sogenannten Gegenpunkte des Punktquadrupels $P_{1} P_{2} P_{3} P_{4}$, in einer Geraden liegen. Dieser Gegenpunkt ist nämlich der dritte Schnittpunkt von $p^{3}$ mit $P_{x} P_{y}$, wo $P_{x}$ und $P_{y}$ die dritten Schnittpunkte von $p^{3}$ mit $P_{1} P_{2}$ und $P_{3} P_{4}$ sind.

74. Mit Hilfe der Sätze 27 und 31 läßt sich auch der folgende Satz leicht beweisen.

Liegen sechs Punkte $P_{1}, P_{2}, P_{3}, P_{4}, P_{5}, P_{6}$ von $p^{3}$ auf einem Kegelschnitt und verbindet man einen dieser Punkte, etwa $P_{1}$, mit den fünf übrigen durch die Geraden $P_{1} P_{2}, P_{1} P_{3}$, $P_{1} P_{4}, P_{1} P_{5}, P_{1} P_{6}$, so liegen die fünf Punkte $P_{12}, P_{13}, P_{14}$, $P_{15}, P_{16}$, in denen jene fünf Geraden der Reihe nach von $p^{3}$ zum drittenmal geschnitten werden, und der zweite Tangentialpunkt von $P_{1}$ auf einem Kegelschnitt.

Denn nach Satz 31 ist:

nach Satz 27:

$$
\begin{gathered}
\omega_{1}+\omega_{2}+\omega_{3}+\omega_{4}+\omega_{5}+\omega_{6}=k \pi, \\
\omega_{1}+\omega_{2}+\omega_{12}=\frac{2 k_{2}+1}{2} \pi \\
\omega_{1}+\omega_{3}+\omega_{13}=\frac{2 k_{3}+1}{2} \pi \\
\omega_{1}+\omega_{4}+\omega_{14}=\frac{2 k_{4}+1}{2} \pi \\
\omega_{1}+\omega_{5}+\omega_{15}=\frac{2 k_{5}+1}{2} \pi \\
\omega_{1}+\omega_{6}+\omega_{16}=\frac{2 k_{6}+1}{2} \pi
\end{gathered}
$$


und nach Nr. 65 und Satz 15 (Nr.26) ist der Ordinatenwinkel des zweiten Tangentialpunktes von $P_{1}$ gleich

mithin ist:

$$
\omega_{1}^{(2)}+\frac{\pi}{2}=4 \omega_{1}+\frac{\pi}{2}
$$

$$
\begin{gathered}
\omega_{12}+\omega_{13}+\omega_{14}+\omega_{15}+\omega_{16}+\left(\omega_{1}^{(2)}+\frac{\pi}{2}\right)=\left(k_{2}+k_{3}+k_{4}+k_{5}\right. \\
\left.+k_{6}\right) \pi+\frac{5}{2} \pi-5 \omega_{1}-\left(\omega_{2}+\omega_{3}+\omega_{4}+\omega_{5}+\omega_{6}\right)+4 \omega_{1}+\frac{\pi}{2} \\
=\left(k_{2}+k_{3}+k_{4}+k_{5}+k_{6}\right) \pi+\frac{5}{2} \pi-k \pi+\frac{\pi}{2}=k^{\prime} \pi,
\end{gathered}
$$

woraus nach Satz 31 der vorstehende Satz folgt.

75. Mit Hilfe der Sätze 28, 29 und 30 können nun die folgenden Aufgaben gelöst werden.

Aufgabe 9. Den sechsten Schnittpunkt von $p^{3}$ mit einem durch fünf ihrer Punkte $P_{1}, P_{2}, P_{3}, P_{4}, P_{5}$ gehenden Kegelschnitt zu bestimmen.

Auflösung. Man ermittelt etwa die vier Pole (in bezug auf $A B C) G_{1}, G_{2}, G_{3}, G_{4}$ der $P_{1}, P_{2}, P_{3}, P_{4}$ von $P$, dem isolierten Doppelpunkt von $p^{3}$, aus projizierenden Geraden $g_{1}$, $g_{2}, g_{3}, g_{4}$ und den Schnittpunkt $Q_{5}$ von $p$, der Polare (in bezug auf $A B C$ ) von $P$, mit $\operatorname{der} P_{5}$ von $P$ aus projizierenden Geraden $g_{5}$; sodann ermittelt man in der auf $p$ durch das Viereck $G_{1} G_{2} G_{3} G_{4}$ festgelegten, auf $(p)^{2}$ sich stützenden Involution den zu $Q_{5}$ zugepaarten Punkt, dieser möge $Q_{6}$ heißen, und verbindet diesen mit $P$ durch eine Gerade $g_{6}$; alsdann wird derjenige Punkt $P_{6}$, welcher in der von $A B C$ auf $g_{6}$ erzeugten Involution $\left(g_{6}\right)^{2} \operatorname{dem} P$ zugepaart ist, der gesuchte sechste Schnittpunkt sein.

Diese Lösung, welche mit Hilfe des Lineals allein ausführbar ist, ergibt sich unmitelbar aus dem Satze 30 und behält ihre Gültigkeit (nach Nr. 72) auch dann noch, wenn mehrere der fünf gegebenen Punkte, ja sogar wenn alle fünf einander unendlich benachbart sind und also (im letztern Falle) der sechste Schnittpunkt von $p^{3}$ mit einem $p^{3}$ fünfpunktig berührenden Kegelschnitt gesucht wird. 
Sind $\omega_{1}, \omega_{2}, \omega_{3}, \omega_{4}, \omega_{5}$ die Ordinatenwinkel von $P_{1}, P_{2}, P_{3}$, $P_{4}, P_{5}$, so ist der Ordinatenwinkel des sechsten Schnittpunktes $P_{6}$ nach Satz 31 gleich $-\left(\omega_{1}+\omega_{2}+\omega_{3}+\omega_{4}+\omega_{5}\right)$.

Aufgabe 10. Die weitern Schnittpunkte von $p^{3}$ mit einem durch zwei voneinander verschiedene Punkte $P_{i}$ und $P_{k}$ von $p^{3}$ gehenden Kegelschnitt $k^{2}$ zu bestimmen.

Auflösung. Man leitet in der in Satz 28 angegebenen Weise aus dem Kegelschnitt $k^{2}$ den durch $Q_{i} \equiv p g_{i}$ und $Q_{k}$ $\equiv p g_{k}$ gehenden Kegelschnitt $x^{2}$ ab und ermittelt die vier Schnittpunkte $G_{1}, G_{2}, G_{3}, G_{4}$ dieses Kegelschnitts mit $p^{2}$, dem Polarkegelschnitt von $P$, und deren durch $P$ gehenden Polaren (in bezug auf $A B C$ ) $g_{1}, g_{2}, g_{3}, g_{4}$, sodann in den auf diesen von $A B C$ erzeugten Involutionen $\left(g_{1}\right)^{2},\left(g_{2}\right)^{2},\left(g_{3}\right)^{2},\left(g_{4}\right)^{2}$ die dem $P$ zugepaarten Punkte $P_{1}, P_{2}, P_{3}, P_{4}$; diese werden dann die gesuchten weitern Schnittpunkte von $p^{3}$ mit $k^{2}$ sein.

Sind außer $P_{i}$ und $P_{k}$ noch ein oder mehrere der Schnittpunkte von $p^{3}$ mit $k^{2}$ bekannt, so sind dem entsprechend ein oder mehrere der Schnittpunkte von $p^{2}$ mit $x^{2}$ gleichfalls bekannt und, um die übrigen der erstern Schnittpunkte zu finden, kommt es nur noch auf die Bestimmung der übrigen der letztern an.

Sind außer $P_{i}$ und $P_{k}$ noch zwei der weitern vier Schnittpunkte von $p^{3}$ mit $k^{2}$, etwa $P_{1}$ und $P_{2}$, bekannt, so ermittelt man in derjenigen auf $(p)^{2}$, der von $A B C$ auf $p$ erzeugten Involution, sich stützenden Involution, von der $Q_{i} Q_{k}$ ein Punktepaar sind, den zu $Q_{x}$, dem Schnittpunkte von $p$ mit $G_{1} G_{2}$, zugepaarten Punkt $Q_{y}$, sodann die beiden Polaren von $Q_{y}$ in bezug auf die Kegelschnitte $p^{2}$ und $x^{2}$ (wobei die Polare von $Q_{y}$ in bezug auf $p^{2}$ nach Nr. 2 die $P$ mit dem zu $Q_{y}$ in $(p)^{2}$ zugepaarten Punkte verbindende Gerade sein wird), verbindet den Schnittpunkt dieser beiden Polaren mit $Q_{y}$ durch eine Gerade und ermittelt endlich die beiden Schnittpunkte dieser Geraden mit $p^{2}$; alsdann werden diese Schnittpunkte die Pole $G_{3}$ und $G_{4}$ der die übrigen Schnittpunkte $P_{3}$ und $P_{4}$ von $p^{3}$ mit $k^{2}$ aus $P$ projizierenden Geraden $g_{3}$ und $g_{4}$ sein.

Denn nach Satz $30 \mathrm{mu} G_{3} G_{4}$, die gemeinschaftliche Sekante von $p^{2}$ und $x^{2}$, durch $Q_{y}$ und mithin auch durch den Schnittpunkt der beiden Polaren von $Q_{y}$ in bezug auf $p^{2}$ und $x^{2}$ gehen. 
A ufgabe 11. Durch zwei voneinander verschiedene Punkte $P_{1}$ und $P_{2}$ von $p^{3}$ denjenigen Kegelschnitt zu legen, welcher in einem der beiden Punkte, etwa in $P_{1}$, von $p^{3}$ dreipunktig berührt wird und außerdem noch durch irgendeinen gegebenen Punkt $P_{3}$ von $p^{3}$ geht.

Auflösung. Man ermittelt von demjenigen Kegelschnitt $x^{2}$, welcher durch $Q_{1} \equiv p g_{1}, Q_{2} \equiv p g_{2}, G_{1}, G_{3}$ geht und in $G_{1}$ von $p^{2}$, dem Polarkegelschnitt von $P$, einfach berührt wird, etwa seine Tangente $v$ in $Q_{1}$, sodann die Pole (in bezug auf $A B C) V, V_{1}$ von $v, v_{1} \equiv Q_{1} G_{1}$; alsdann wird derjenige Kegelschnitt $k^{2}$, welcher durch die beiden projektiven Strahlenbüschel

$$
P_{1}\left(V, V_{1}, P_{3}, \ldots\right) \pi P_{2}\left(P, P_{1}, P_{3}, \ldots\right)
$$

erzeugt wird, durch $P_{1}, P_{2}$ und $P_{3}$ gehen und in $P_{1}$ von $p^{3}$ dreipunktig berührt werden.

Denn der erstere Kegelschnitt, nämlich $\varkappa^{2}$, hat mit dem aus $k^{2}$ in der in Satz 28 angegebenen Weise abgeleiteten Kegelschnitt, welcher letzte durch

$$
Q_{1}\left(v, v_{1}, Q_{1} G_{3}, \ldots\right) \pi Q_{2}\left(p, Q_{2} G_{1}, Q_{2} G_{3}, \ldots\right)
$$

erzeugt wird, die vier Punkte $Q_{1}, Q_{2}, G_{1}, G_{3}$ und die Tangente $v$ in $Q_{1}$ (da $p \equiv Q_{2} Q_{1}$ ist) gemein und ist folglich mit diesem identisch; mithin muß nach Satz $29 k^{2}$ von $p^{3}$ in $P_{1}$ dreipunktig berührt werden, da $x^{2}$ von $p^{2}$ in $G_{1}$ einfach berührt wird.

Aufgabe 12. Durch zwei voneinander verschiedene Punkte $P_{1}$ und $P_{2}$ von $p^{3}$ denjenigen Kegelschnitt zu legen, welcher in einem derselben, etwa in $P_{1}$, von $p^{3}$ vierpunktig berührt wird.

Auflösung. Man ermittelt in derjenigen auf $(p)^{2}$ sich stützenden Involution $(i)^{2}$, in der $Q_{1} Q_{2}$ ein Punktepaar bilden, den zu dem Schnittpunkte $p t_{1}$ (wo $t_{1}$ die Tangente von $p^{2}$ in $G_{1}$ ist) zugepaarten Punkt, verbindet diesen mit $G_{1}$ durch eine Gerade und ermittelt den zweiten Schnittpunkt $G_{4}$ dieser Geraden mit $p^{2}$; sodann ermittelt man im Punkte $Q_{1}$ die Tangente $v$ desjenigen Kegelschnitts $x^{2}$, welcher durch $Q_{1}, Q_{2}$, $G_{1}, G_{4}$ geht und in $G_{1}$ dieselbe Tangente $t_{1}$ hat, wie $p^{2}$, und die Pole (in bezug auf $A B C$ ) $V, V_{1}, V_{4}, W_{4}$ von $v, v_{1} \equiv Q_{1} G_{1}$, Berliner, Habilitationsschrift. 
$v_{4} \equiv Q_{1} G_{4}, w_{4} \equiv Q_{2} G_{4} ;$ alsdann wird derjenige Kegelschnitt $k^{2}$, welcher durch die beiden projektiven Strahlenbüschel

$$
P_{1}\left(V, V_{1}, V_{4}, \ldots\right) \pi P_{2}\left(P, P_{1}, W_{4}, \ldots\right)
$$

erzeugt wird, durch $P_{1}$ und $P_{2}$ gehen und in $P_{1}$ von $p^{3}$ vierpunktig berührt werden.

Denn derjenige Kegelschnitt, welcher durch $G_{1}, G_{4}, Q_{1}$ geht und in $G_{1}$ von $p^{2}$ dreipunktig berührt wird, muß auch durch $Q_{2}$ gehen (s. oben Nr. 72) und also mit $x^{2}$ identisch sein, da sie die vier Punkte $Q_{1}, Q_{2}, G_{1}, G_{4}$ und die Tangente $t_{1}$ in $G_{1}$ gemein haben; $x^{2}$ ist aber mit dem aus $k^{2}$ abgeleiteten identisch. Mithin muß (nach Satz 29) $k^{2}$ in $P_{1}$ von $p^{3}$ vierpunktig berührt werden.

Dieses Verfahren versagt aber, wenn der Schnittpunkt $p t_{1}$ einer der Doppelpunkte von $(i)^{2}$ ist, da dann $G_{4}$ mit $G_{1}$ zusammenfallen wird und wir werden die zur Bestimmung von $x^{2}$ nötigen fünf Elemente nicht mehr haben; alsdann wird aber, wie wir bald sehen werden, derjenige durch $Q_{1}, Q_{2}, G_{1}$ gehende Kegelschnitt, welcher in $G_{1}$ von $p^{2}$ mehr als zweipunktig berührt wird, notwendig von $p^{2}$ in $G_{1}$ vierpunktig berührt werden. Dieser Fall, nämlich, daß $p t_{1}$ ein Doppelpunkt von $(i)^{2}$ ist, tritt dann und nur dann ein, wenn der dritte Schnittpunkt von $p^{3}$ mit $P_{1} P_{2}$ der zweite Tangentialpunkt von $P_{1}$ ist. Denn die auf $(p)^{2}$ sich stützende Punktinvolution $(i)^{2}$ wird von $P$ aus durch eine auf $(P)^{2}$ sich stützende Strahleninvolution $(I)^{2}$ projiziert; und durch die Strahlenpaare von $(I)^{2}$, von denen eins $g_{1} g_{2}$ ist (da $Q_{1} Q_{2}$ ein Punktepaar von $(i)^{2}$ ist), werden nun (nach Nr. 63) solche Punktepaare auf $p^{3}$ projiziert, die mit einem und demselben Punkte von $p^{3}$, nämlich mit dem gemeinsamen Tangentialpunkte der beiden von $P$ aus durch die Doppelstrahlen von $(I)^{2}$ projizierten Punkte von $p^{3}$, in je einer Geraden liegen und von denen eins $P_{1} P_{2}$ ist. Ferner geht der $P$ mit $p t_{1}$ verbindende Strahl durch den Tangentialpunkt von $P_{1}$ (nach II in Nr. 55). Mithin ist $p t_{1}$ dann und nur dann ein Doppelpunkt von $(i)^{2}$, wenn ein Doppelstrahl von $(I)^{2}$ den Tangentialpunkt von $P_{1}$ aus $P$ projiziert, wenn also derjenige Punkt von $p^{3}$, mit welchem alle durch Strahlenpaare von $(I)^{2}$ projizierten Punktepaare auf $p^{3}$, darunter auch $P_{1} P_{2}$, in je einer Geraden liegen, der Tangentialpunkt jenes Tangentialpunktes von $P_{1}$, also der zweite Tangentialpunkt von $P_{1}$ ist. 
Aufgabe 13. Durch zwei voneinander verschiedene Punkte $P_{1}$ und $P_{2}$ von $p^{3}$ denjenigen Kegelschnitt zu legen, welcher in einem derselben, etwa in $P_{1}$, von $p^{3}$ fünfpunktig berührt wird; was aber dann und nur dann möglich ist, wenn $P_{1} P_{2}$ durch den zweiten Tangentialpunkt von $P_{1}$ geht.

Denn nach Satz 30 und Nr. 72 geht der $p^{3}$ in $P_{1}$ fünfpunktig berührende Kegelschnitt dann und nur dann durch $P_{2}$, wenn $Q_{1} Q_{2}$ ein Punktepaar in derjenigen auf $(p)^{2}$ sich stützenden Involution bilden, von der der Schnittpunkt $p t_{1}$ (wo $t_{1}$ die Tangente von $p^{2}$ in $G_{1}$ ist) ein Doppelpunkt ist; dies ist aber, wie wir eben sahen, dann und nur dann der Fall, wenn $P_{1} P_{2}$ durch den zweiten Tangentialpunkt von $P_{1}$ geht.

Dasselbe ergibt sich auch aus dem Satze 32. Denn wenn $P_{2}$ auf dem $p^{3}$ in $P_{1}$ fünfpunktig berührenden Kegelschnitt liegt, so ist dann und nur dann (Satz 32):

$$
5 \omega_{1}+\omega_{2}=k \pi,
$$

also ist nur dann:

$$
\omega_{1}+\omega_{2}+\left(\omega_{1}^{(2)}+\frac{\pi}{2}\right)=k \pi-4 \omega_{1}+4 \omega_{1}+\frac{\pi}{2}=\frac{2 k+1}{2} \pi ;
$$

mithin (nach Satz 27 in Nr. 64) geht dann und nur dann $P_{1} P_{2}$ durch den zweiten Tangentialpunkt von $P_{1}$.

Auflösung. Man ermittelt in einer auf $(p)^{2}$ sich stützenden Involution, in der $Q_{1} Q_{x}$ ein Punktepaar bilden (wo $Q_{x}$ irgendein beliebiger, von $Q_{2}$ aber verschiedener Punkt von $p$ ist), den zu dem Schnittpunkte $p t_{1}$ (wo $t_{1}$ die Tangente von $p^{2}$ in $G_{1}$ ist) zugepaarten Punkt, verbindet diesen mit $G_{1}$ durch eine Gerade und ermittelt auf dieser Geraden ihren zweiten Schnittpunkt $G_{3}$ mit $p^{2}$. Sodann zieht man durch $Q_{2}$ eine beliebige Gerade $l$ und ermittelt ihre beiden Schnittpunkte $M$ und $N$ mit demjenigen Kegelschnitt $x_{x}^{2}$, welcher durch $Q_{1}, Q_{x}, G_{1}, G_{3}$ geht und in $G_{1}$ von $t_{1}$ tangiert wird. Ferner ermittelt man in derjenigen Involution auf 7 , von der $M N$ und die beiden Schnittpunkte von $l$ mit $t_{1}$ und $G_{1} Q_{1}$ zwei Punktepaare sind, den zu $Q_{2}$ zugepaarten Punkt, er heiße etwa $L$; darauf ermittelt man in $Q_{1}$ die Tangente $v$ desjenigen Kegelschnitts $x^{2}$, welcher durch $Q_{1}, Q_{2}, G_{1}, L$ geht und in $G_{1}$ von $t_{1}$ tangiert wird, und 
die Pole (in bezug auf $A B C$ ) $V, V_{1}, V_{2}, W_{2}$ von $v, v_{1} \equiv Q_{1} G_{1}$, $v_{2} \equiv Q_{1} L, w_{2} \equiv Q_{2} L$; alsdann wird derjenige Kegelschnitt $k^{2}$, welcher durch die beiden projektiven Strahlenbüschel

$$
P_{1}\left(V, V_{1}, V_{2}, \ldots\right) \pi P_{2}\left(P, P_{1}, W_{2}, \ldots\right)
$$

erzeugt wird, durch $P_{1}$ und $P_{2}$ gehen und in $P_{1}$ von $p^{3}$ fünfpunktig berührt werden.

Denn der Kegelschnitt $x_{x}^{2}$ wird, wie wir in der Auflösung der Aufgabe 12 sahen, von $p^{2}$ in $G_{1}$ dreipunktig berührt. Nunmehr muß derjenige Kegelschnitt, welcher durch $G_{1}, Q_{1}, Q_{2}$ geht und in $G_{1}$ von $x_{x}^{2}$ dreipunktig berührt wird, auch durch den zu $Q_{2}$ in der genannten Involution auf $l$ zugepaarten Punkt $L$ gehen (s. oben Nr. 72) und also mit $x^{2}$ identisch sein, da sie die vier Punkte $G_{1}, Q_{1}, Q_{2}, L$ und die Tangente $t_{1}$ in $G_{1}$ gemein haben; mithin muß $x^{2}$ von $x_{x}^{2}$ und also auch von $p^{2}$ in $G_{1}$ dreipunktig berührt werden. Weil aber der Kegelschnitt, welcher durch $G_{1}$ und $Q_{1}$ geht und in $G_{1}$ von $p^{2}$ vierpunktig berührt wird, auch durch denjenigen Punkt geht, welcher in der auf $(p)^{2}$ sich stützenden Involution $(i)^{2}$, deren einer Doppelpunkt $p t_{1}$ ist, zu $Q_{1}$ zugepaart ist, und welcher Punkt also, weil $P_{1} P_{2}$ durch den zweiten Tangentialpunkt von $P_{1}$ geht, wie wir sahen, $Q_{2}$ sein muß, und somit mit $x^{2}$, der durch $G_{1}, Q_{1}, Q_{2}$ gehende und $p^{2}$ in $G_{1}$ dreipunktig berührende Kegelschnitt, identisch ist, so wird $x^{2}$ von $p^{2}$ in $G_{1}$ vierpunktig und mithin (nach Satz 29) $k^{2}$ von $p^{3}$ in $P_{1}$ fünfpunktig berührt.

Aufgabe 14. Vier beliebige Punkte $P_{1}, P_{2}, P_{3}, P_{4}$ von $p^{3}$ seien gegeben, es soll ein solcher Punkt von $p^{3}$ ermittelt werden, welcher ein Berührungspunkt eines durch die vier gegebenen Punkte gehenden Kegelschnitts mit $p^{3}$ sein soll.

Auflösung. Man ermittelt die Doppelpunkte der auf $p$ durch das Viereck $G_{1} G_{2} G_{3} G_{4}$ festgelegten, auf $(p)^{2}$ sich stützenden Involution, verbindet diese Doppelpunkte mit $P$ durch zwei Strahlen, welche ein Strahlenpaar in $(P)^{2}$ bilden, und ermittelt in den auf diesen zwei Strahlen von $A B C$ erzeugten Involutionen zweiten Grades die $\mathrm{zu}$ $P$ zugepaarten Punkte; jeder dieser beiden Punkte und nur einer dieser beiden liefert eine Lösung der gestellten Aufgabe. 
Die Ordinatenwinkel der beiden soeben gefundenen Punkte sind nach Satz 32 (für $k=0,1$ ):

$$
-\frac{\omega_{1}+\omega_{2}+\omega_{3}+\omega_{4}}{2} \text { und } \frac{\pi-\left(\omega_{1}+\omega_{2}+\omega_{3}+\omega_{4}\right)}{2} \text {. }
$$

(Diese Lösung folgt unmittelbar aus Satz 30 nach Nr. 72.)

Die gestellte Aufgabe hat also im allgemeinen zwei und nur zwei eigentliche Lösungen, nur wenn $P_{1}, P_{2}, P_{3}, P_{4}$ vier aufeinanderfolgende Punkte von $p^{3}$ sind, oder wenn zweimal zwei dieser Punkte aufeinander folgen, oder endlich wenn ein und nur ein Diagonalpunkt des vollständigen Vierecks $P_{1} P_{2} P_{3} P_{4}$ auf $p^{3}$ liegt, hat die Aufgabe nur eine eigentliche Lösung, die zweite liefert dann einen in ein Geradenpaar ausartenden Kegelschnitt, und nur wenn zwei der Diagonalpunkte von $P_{1} P_{2} P_{3} P_{4}$ auf $p^{3}$ liegen, wenn also (Nr. 67) aus $P_{1}, P_{2}, P_{3}, P_{4}$ solche zwei Punktepaare gebildet werden können, welche von $P$ aus durch zwei Strahlenpaare von $(P)^{2}$ projiziert werden, hat die Aufgabe keine eigentlichen Lösungen.

Denn wenn $P_{1}, P_{2}, P_{3}, P_{4}$ vier aufeinanderfolgende Punkte von $p^{3}$ sind, so sind auch $G_{1}, G_{2}, G_{3}, G_{4}$ vier aufeinanderfolgende Punkte von $p^{2}$ und die Tangente $t_{1}$ von $p^{2}$ in $G_{1}$ geht (Nr. 72) durch einen Doppelpunkt der auf $p$ durch $G_{1} G_{2} G_{3} G_{4}$ festgelegten, auf $(p)^{2}$ sich stützenden Involution; der diesen Doppelpunkt mit $P$ verbindende Strahl projiziert nun (nach II in Nr. 55) den Tangentialpunkt von $P_{1}$ und der hierdurch gelieferte Kegelschnitt muß ein ausgearteter sein. Ebenso wenn etwa $P_{1}, P_{2}$ und $P_{3}, P_{4}$ je zwei aufeinanderfolgende Punkte sind, sind auch $G_{1}, G_{2}$ und $G_{3}, G_{4}$ je zwei aufeinanderfolgende Punkte von $p^{2}$ und $G_{1} G_{3} \equiv G_{2} G_{4}$ geht (Nr. 72) durch einen Doppelpunkt der erwähnten Involution; der diesen Doppelpunkt mit $P$ verbindende Strahl projiziert nun (nach II) den dritten Schnittpunkt von $p^{3}$ mit $P_{1} P_{3} \equiv P_{2} P_{4}$ und der hierdurch gelieferte Kegelschnitt muß wiederum ein ausgearteter sein. Wenn ferner ein uud nur ein Diagonalpunkt von $P_{1} P_{2} P_{3} P_{4}$ auf $p^{3}$ liegt, so liegt dann (nach II) ein und nur ein Diagonalpunkt von $G_{1} G_{2} G_{3} G_{4}$ auf $p$ und dieser Diagonalpunkt ist dann ein Doppelpunkt der auf $p$ durch $G_{1} G_{2} G_{3} G_{4}$ festgelegten Involution; der diesen Doppelpunkt mit $P$ verbindende Strahl wird dann jenen auf $p^{3}$ liegenden Diagonalpunkt von 
$P_{1} P_{2} P_{3} P_{4}$ projizieren. Endlich wenn zwei der Diagonalpunkte von $P_{1} P_{2} P_{3} P_{4}$ auf $p^{3}$ liegen, so liegen dann (nach II) zwei der Diagonalpunkte von $G_{1} G_{2} G_{3} G_{4}$ auf $p$ und sie sind die Doppelpunkte der genannten Involution; die diese beiden Doppelpunkte mit $P$ verbindenden Strahlen projizieren dann jene beiden auf $p^{3}$ liegenden Diagonalpunkte von $P_{1} P_{2} P_{3} P_{4}$, und die beiden hierdurch gelieferten Kegelschnitte müssen dann ausgeartete sein.

Zugleich haben wir für die speziellen Fälle die folgenden Lösungen gewonnen.

Aufgabe 15. Es soll auf $p^{3}$ derjenige Punkt gefunden werden, welcher ein einfacher Berührungspunkt von $p^{3}$ mit einem dieselbe Kurve $p^{3}$ in einem gegebenen Punkte $P_{1}$ vierpunktig berührenden Kegelschnitt sein soll.

Auflösung. Man ermittelt den ersten Repräsentanten $g_{1}^{(1)} \equiv P G_{1}$ von $g_{1} \equiv P P_{1}$, sodann in der auf $g_{1}^{(1)}$ von $A B C$ erzeugten Involution $\left(g_{1}^{(1)}\right)^{2}$ den zu $P$ gepaarten Punkt; dieser letztere Punkt wird dann der gesuchte sein.

Der Ordinatenwinkel des gesuchten Punktes ist:

$$
\omega_{1}^{(1)}=-2 \omega_{1} \text {. }
$$

Denn, wie wir eben sahen, wird von demjenigen Strahlenpaare von $(P)^{2}$, welches die Doppelpunkte der auf $p$ durch das Viereck der vier in $G_{1}$ aufeinanderfolgenden Punkte festgelegten, auf $(p)^{2}$ sich stützenden Involution projiziert, der eine Strahl von $P$ aus den Tangentialpunkt von $P_{1}$ projizieren und mithin (nach Nr. 65) muß der zweite Strahl des Paares der erste Repräsentant von $g_{1}$ sein.

A ufgabe 16. Es seien irgend zwei Punkte $P_{1}$ und $P_{2}$ von $p^{3}$ gegeben, es soll derjenige Punkt auf $p^{3}$ gefunden werden, welcher zusammen mit $P_{1}$ und $P_{2}$ solche drei Punkte bildet, durch die ein $p^{3}$ in jedem derselben einfach berührender Kegelschnitt geht.

Auflösung. Man ermittelt die Pole $G_{1}$ und $G_{2}$ von $g_{1}$ und $g_{2}$, sodann in der von $A B C$ erzeugten Involution $(p)^{2}$ den zum Schnittpunkte $\left(p, G_{1} G_{2}\right)$ zugepaarten Punkt, verbindet diesen mit $P$ durch eine Gerade und ermittelt in der auf dieser Geraden von $A B C$ erzeugten Involution zweiten Gerades den zu $P$ zugepaarten Punkt; dieser letzte Punkt wird dann der gesuchte sein. 
Der Ordinatenwinkel des gesuchten Punktes ist (nach Satz 32): $-\left(\omega_{1}+\omega_{2}\right)$.

Ganz analog ist zu verfahren, wenn etwa $P_{1} P_{2}$ und $P_{3} P_{4}$ auf $p^{3}$ sich schneiden.

Aufgabe 17. Drei beliebige Punkte $P_{1}, P_{2}, P_{3}$ von $p^{3}$ seien gegeben, es soll ein solcher Punkt auf $p^{3}$ ermittelt werden, welcher ein (zweiter) Berührungspunkt von $p^{3}$ mit einem durch $P_{1}$ und $P_{2}$ gehenden und außerdem $p^{3}$ in $P_{3}$ einfach berührenden Kegelschnitt sein soll.

Auflösung 1. Man ermittelt die Pole $G_{1}, G_{2}, G_{3}$ von $g_{1}, g_{2}, g_{3}$, sodann die Berührungspunkte, die etwa $G_{m}$ und $G_{n}$ heißen mögen, der beiden aus dem Schnittpunkte $\left(p, G_{1} G_{2}\right)$ an $p^{2}$, den Polarkegelschnitt von $P$, gehenden Tangenten und ihre durch $P$ gehenden Polaren (in bezug auf $A B C) g_{m}$ und $g_{n}$, bringt $g_{m}$ und $g_{n}$ zum Schnitt mit $p$ in $Q_{m}$ und $Q_{n}$ und ermittelt die zweiten Schnittpunkte, die etwa $G_{k}$ und $G_{l}$ heißen mögen, von $p^{2}$ mit $Q_{m} G_{3}$ und $Q_{n} G_{3}$ und ihre durch $P$ gehenden Polaren (in bezug auf $A B C$ ) $g_{k}$ und $g_{l}$; alsdann wird jeder der beiden Punkte $P_{k}$ und $P_{l}$ und nur einer dieser beiden, welche in den von $A B C$ erzeugten Involutionen $\left(g_{k}\right)^{2}$ und $\left(g_{l}\right)^{2}$ zu $P$ zugepaart sind, der in der Aufgabe gestellten Forderung genügen.

Denn soll etwa durch $P_{1}, P_{2}, P_{3}, P_{x}$ ein solcher Kegelschnitt gehen, welcher von $p^{3}$ in $P_{3}$ und $P_{x}$ je einfach berührt werden soll, so werden dann und nur dann (nach Satz 30 und Nr. 72) $g_{3}$ und $g_{x}$ durch ein Punktepaar der auf $p$ durch $G_{1} G_{2} G_{3} G_{x}$ festgelegten, auf $(p)^{2}$ sich stützenden Involution $(i)^{2}$ gehen und mithin $g_{3} g_{x}$ ein Paar in der $(i)^{2}$ von $P$ aus projizierenden, auf $(P)^{2}$ sich stützenden Strahleninvolution $(I)^{2}$ bilden. Bezeichnen wir dann mit $Q_{12}$ und $Q_{y}$ die Schnittpunkte von $p$ mit $G_{1} G_{2}$ und $G_{3} G_{x}$, so werden auch $g_{12}$ und $g_{y}$, welche das Punktepaar $Q_{12} Q_{y}$ von $(i)^{2}$ und zugleich (nach II in Nr. 55) $P_{12}$ und $P_{y}$, die dritten Schnittpunkte von $p^{3}$ mit $P_{1} P_{2}$ und $P_{3} P_{x}$, von $P$ aus projizieren, ein Strahlenpaar in $(I)^{2}$ bilden müssen. Es wird also dann und nur dann nach Nr. 63 (weil $g_{3} g_{x}$ ein Paar in der auf $(P)^{2}$ sich stützenden Strahleninvolution $(I)^{2}$ bilden) $P_{y}$, der dritte Schnittpunkt von $p^{3}$ mit $P_{3} P_{x}$, derjenige Punkt sein, mit welchem alle die durch Strahlenpaare von $(I)^{2}$ aus $P$ projizierten 
Punktepaare von $p^{3}$ in je einer Geraden liegen, und mithin (Nr.63) $P_{12}$, der zusammen mit $P_{y}$ durch ein Strahlenpaar von $(I)^{2}$ projizierte Punkt von $p^{3}$, der Tangentialpunkt von $P_{y}$. Folglich wird dann und nur dann (nach II) die Tangente von $p^{2}$ in $G_{y}$, dem Pole von $g_{y}$, durch $Q_{12} \equiv\left(p, G_{1} G_{2}\right)$ gehen und mithin $G_{y}$ mit einem der beiden Berïhrungspunkte $G_{m}$ und $G_{n}$ der beiden von $\left(p, G_{1} G_{2}\right)$ aus an $p^{2}$ gehenden Tangenten, $g_{y}$ mit $g_{m}$ oder $g_{n}$, $Q_{y} \equiv p g_{y} \quad$ mit $\quad Q_{m} \equiv p g_{m}$ oder $Q_{n} \equiv p g_{n}, \quad G_{x} \equiv\left(p^{2}, Q_{y} G_{3}\right) \quad$ mit $G_{k} \equiv\left(p^{2}, Q_{m} G_{3}\right)$ oder $G_{l} \equiv\left(p^{2}, Q_{n} G_{3}\right), g_{x}$ mit $g_{k}$ oder $g_{l}$ und endlich $P_{x}$ mit $P_{k}$ oder $P_{l}$ identisch sein.

Auflösung 2. Man ermittelt den Pol $G_{3}$ von $g_{3}$, legt die Tangente $t_{3}$ an $p^{2}$ in $G_{3}$ und ermittelt in derjenigen auf $(p)^{2}$ sich stützenden Involution, von $\operatorname{der} Q_{1} Q_{2}\left(Q_{1} \equiv p g_{1}\right.$, $Q_{2} \equiv p g_{2}$ ) ein Punktepaar sind, den zum Schnittpunkte $p t_{3}$ zugepaarten Punkt, sodann die Berührungspunkte $G_{k}$ und $G_{l}$ der beiden aus dem letzt gefundenen Punkte an $p^{2}$ gehenden Tangenten und die durch $P$ gehenden Polaren (in bezug auf $A B C$ ) $g_{k}$ und $g_{l}$ von $G_{k}$ und $G_{l}$; alsdann wird jeder der beiden Punkte $P_{k}$ und $P_{l}$ und nur einer dieser beiden, welche zu $P$ zugepaart sind in den von $A B C$ erzeugten Involutionen $\left(g_{k}\right)^{2}$ und $\left(g_{l}\right)^{2}$, der in der Aufgabe gestellten Forderung genügen.

Denn soll etwa durch $P_{1}, P_{2}, P_{3}, P_{x}$ ein solcher Kegelschnitt gehen, welcher von $p^{3}$ in $P_{3}$ und $P_{x}$ je einfach berührt werden soll, so werden dann und nur dann (nach Satz 30 und Nr. 72) die Schnittpunkte $Q_{33}$ und $Q_{x x}$ von $p$ mit $t_{3}$ und $t_{x}$, den Tangenten an $p^{2}$ in $G_{3}$ und $G_{x}$, ein Punktepaar in derjenigen auf $(p)^{2}$ sich stützenden Involution $(i)^{2}$ bilden, in der $Q_{1} Q_{2}$ gleichfalls ein Punktepaar bilden; mithin wird dann und nur dann $G_{x}$ mit einem der beiden Berührungspunkte $G_{k}$ und $G_{l}$ der beiden aus $Q_{x x}$, dem in $(i)^{2} \mathrm{zu} Q_{33}$ zugepaarten Punkte, an $p^{2}$ gehenden Tangenten und also $P_{x}$ mit einem der beiden Punkte $P_{k}$ und $P_{l}$ identisch sein.

Diese beiden Lösungen sind auch dann noch anzuwenden, wenn auf dem Berührungspunkte $P_{3}$ einer der beiden übrigen, etwa $P_{2}$, gleich folgt, wenn also $P_{3}$ ein dreipunktiger Berührungspunkt von $p^{3}$ mit dem Kegelschnitt sein soll; nur wird dann $P_{3}$ auch die Stelle von $P_{2}$ und also $G_{3}$ auch die Stelle von $G_{2}$ vertreten. 
Außer diesen beiden Lösungen kann die Aufgabe 17, die nur ein Spezialfall der Aufgabe 14 darbietet, auch wie diese, die allgemeinere, gelöst werden.

Aufgabe 18. Drei beliebige Punkte $P_{1}, P_{2}, P_{3}$ von $p^{3}$ seien gegeben, es soll ein solcher Punkt auf $p^{3}$ gefunden werden, welcher ein dreipunktiger Berührungspunkt eines durch die drei gegebenen Punkte gehenden Kegelschnitts mit $p^{3}$ sein soll.

Auflösung. Man ermittelt etwa den $\mathrm{Pol} G_{3}$ von $g_{3}$, bezieht den Büschel der Tangenten um $p^{2}$, den Polarkegelschnitt von $P$, auf die Punktreihe auf $p$ in der Weise, daß einer Tangente $t_{i}$, deren Berührungspunkt mit $p^{2} G_{i}$ ist, derjenige Punkt auf $p$ entsprechen soll, welcher zu dem Schnittpunkte $\left(p, G_{3} G_{i}\right)$ zugepaart ist in derjenigen auf $(p)^{2}$ sich stïtzenden Involution $(i)^{2}$, in der $Q_{1} Q_{2}\left(Q_{1} \equiv p g_{1}, Q_{2} \equiv p g_{2}\right)$ ein Punktepaar bilden, und ermittelt sodann diejenigen drei einzig vorhandenen Tangenten $t_{i_{1}}, t_{i_{2}}, t_{i_{3}}$ von $p^{2}$, von denen jede durch den entsprechenden Punkt von $p$ geht (und welche drei Tangenten sämtlich reell sind, wenn auch nur einer der drei gegebenen Punkte reell und die übrigen beiden konjugiertimaginär sind), und die durch $P$ gehenden Polaren (in bezug auf $A B C$ ) $g_{i_{1}}, g_{i_{2}}, g_{i_{3}}$ der Berührungspunkte $G_{i_{1}}, G_{i_{2}}, G_{i_{3}}$ von $t_{i_{1}}, t_{i_{2}}, t_{i_{3}}$ mit $p^{2}$, welche drei Polaren ein Tripel in der von $A B C$ erzeugten Involution $(P)^{3}$ bilden, und also, wenn deren einer bekannt ist, die beiden übrigen mit bestimmt sind; alsdann wird jeder der drei Punkte $P_{i_{1}}, P_{i_{2}}, P_{i_{3}}$ und nur einer dieser drei, welche zu $P$ zugepaart sind in den von $A B C$ erzeugten Involutionen $\left(g_{i_{1}}\right)^{2},\left(g_{i_{2}}\right)^{2}$ und $\left(g_{i_{3}}\right)^{2}$, der in der Aufgabe gestellten Forderung genügen.

Dies erhellt folgendermaßen: Verbindet man $G_{3}$ mit irgendeinem zweiten Punkte $G_{i}$ von $p^{2}$ und läßt $G_{i}$ den ganzen Polarkegelschnitt $p^{2}$ durchlaufen, so beschreibt $G_{3} G_{i}$ einen Strahlenbüschel um $G_{3}$, welcher einerseits zu dem von $t_{i}$, der Tangente an $p^{2}$ in $G_{i}$, um $p^{2}$ beschriebenen Tangentenbüschel projektiv und andererseits zu der von der Spur von $G_{3} G_{i}$ auf $p$ beschriebenen Punktreihe perspektiv und mithin zu derjenigen Punktreihe auf $p$, welche von dem in $(i)^{2}$ zu dem Schnittpunkte $\left(p, G_{3} G_{i}\right)$ zugepaarten Punkte beschrieben wird, projektiv ist. Folglich ist 
auch der Tangentenbüschel $p^{2}\left(t_{i}\right)$ zu der letztern Punktreihe auf $p$ projektiv und es gehen bekanntlich drei und nur drei Tangenten $t_{i_{1}}, t_{i_{2}}, t_{i_{3}}$ von $p^{2}$ durch die entsprechenden Punkte der projektiven Punktreihe, wo von den drei Tangenten $t_{i_{1}}, t_{i_{2}}, t_{i_{3}}$ mindestens eine bekanntlich reell sein muß, wenn auch $P_{1}$ und $P_{2}$ und mithin $Q_{1}$ und $Q_{2}$ konjugiert-imaginär sein sollen, wenn nur $P_{3}$ und mithin $G_{3}$ reell ist, wo also reellen Tangenten von $p^{2}$ reelle Punkte von $p$ entsprechen. Diese drei Tangenten $t_{i_{1}}, t_{i_{2}}, t_{i_{3}}$ müssen aber, wie wir sofort sehen werden, sämtlich reell sein, und zwar durch ein Punktetripel der von $A B C$ auf $p$ erzeugten Involution $(p)^{3}$ gehen. Bilden nämlich $G_{x}, G_{y}, G_{z}$ ein solches Punktetripel in der durch $(P)^{3}$ auf $p^{2}$ induzierten Involution $\left(p^{2}\right)^{3}$ (s. oben Nr. 7), dessen Sinn mit dem Sinne $A B C$ auf $p^{2}$ übereinstimmt, so werden die Spuren $Q_{3 x}, Q_{3 y}, Q_{3 z}$ der drei Geraden $G_{3} G_{x}, G_{3} G_{y}, G_{3} G_{z}$ auf $p$ (weil $G_{3}$ auf $p^{2}$ liegt) ein solches Tripel in $(p)^{3}$ bilden, dessen Sinn zum Sinne $P_{a} P_{b} P_{c}$ auf $p$ entgegengesetzt ist (Nr. 12). Sind nun $Q_{x}^{\prime}, Q_{y}^{\prime}, Q_{z}^{\prime}$ die in $(i)^{2}$ der Reihe nach zu $Q_{3 x}, Q_{3 y}, Q_{3 z}$ zugepaarten Punkte, so muß auch $Q_{x}^{\prime} Q_{y}^{\prime} Q_{z}^{\prime}$ ein Tripel von $(p)^{3}$ sein, und zwar ein solches, dessen Sinn mit $P_{a} P_{b} P_{c}$ übereinstimmt. Denn die auf $(p)^{2}$ sich stützende Involution $(i)^{2}$ wird aus demjenigen Punkte $R$, aus dem $(p)^{2}$ und $(p)^{3}$ durch eine rechtwinklige Strahleninvolution bzw. durch eine Involution der regelmäßigen Dreistrahlen projiziert werden, durch eine symmetrische Strahleninvolution projiziert; mithin müssen die drei Strahlen $R Q_{x}^{\prime}, R Q_{y}^{\prime}$ $R Q_{z}^{\prime}$ ebenso wie die drei Strahlen $R Q_{3 x}, R Q_{3 y}, R Q_{3 z}$ miteinander Winkel von je $60^{\circ}$ bilden, und folglich bilden $Q_{x}^{\prime} Q_{y}^{\prime} Q_{z}^{\prime}$ ein solches Tripel in $(p)^{3}$, dessen Sinn zu $Q_{3 x} Q_{3 y} Q_{3 z}$ entgegengesetzt ist und also mit dem Sinne $P_{a} P_{b} P_{c}$ übereinstimmt. Weil ferner die drei Geraden, nämlich die Tangente $t_{x}$ an $p^{2}$ in $G_{x}, G_{x} G_{y}$ und $G_{x} G_{g}$, welche drei Gerade das Tripel $G_{x} G_{y} G_{z}$ von $\left(p^{2}\right)^{3}$ aus $G_{x}$ projizieren, von $p$ in einem solchen Tripel von $(p)^{3}$ geschnitten werden, dessen Sinn zu $P_{a} P_{b} P_{c}$ entgegengesetzt ist, und weil durch die Schnittpunkte von $p$ mit $G_{x} G_{y}$ und $G_{x} G_{z}$ der Reihe nach $t_{z}$ und $t_{y}$, die Tangenten an $p^{2}$ in $G_{z}$ und $G_{y}$, gehen (Nr. 15), so müssen die Schnittpunkte von $p$ mit $t_{x}, t_{y}, t_{z}$ ein solches Tripel in $(p)^{3}$ bilden, dessen Sinn mit $P_{a} P_{b} P_{c}$ übereinstimmt. Folglich müssen $t_{y}$ und $t_{z}$, die Tangenten an $p^{2}$ in $G_{y}$ und $G_{z}$, der Reihe nach durch $Q_{y}^{\prime}$ und $Q_{z}^{\prime}$, die in $(i)^{2}$ zu $Q_{3 y} \equiv\left(p, G_{3} G_{y}\right)$ und $Q_{3 z} \equiv\left(p, G_{3} G_{z}\right)$ zugepaarten Punkte, gehen, wenn $t_{x}$, die Tangente an $p^{2}$ in $G_{x}$, 
durch $Q_{x}^{\prime}$, den in $(i)^{2}$ zu $Q_{3 x} \equiv\left(p, G_{3} G_{x}\right)$ zugepaarten Punkt, gehen soll; da das Tripel $p\left(t_{x} t_{y} t_{z}\right)$ von $(p)^{3}$, welches dann mit dem Tripel $Q_{x}^{\prime} Q_{y}^{\prime} Q_{z}^{\prime}$ gleichen Sinn und außerdem einen Punkt, nämlich $Q_{x}^{\prime} \equiv p t_{x}$, gemein hat, mit demselben auch dem Sinne nach identisch sein muß und also $Q_{y}^{\prime} \equiv p t_{y}, Q_{z}^{\prime} \equiv p t_{z}$. Hiernach müssen die drei einzig vorhandenen Tangenten $t_{i_{1}}, t_{i_{2}}, t_{i_{3}}$ von $p^{2}$ in $G_{i_{1}}, G_{i_{2}}, G_{i_{3}}$, welche Tangenten durch die in $(i)^{2}$ zu $\left(p, G_{3} G_{i_{1}}\right),\left(p, G_{3} G_{i_{2}}\right)$, $\left(p, G_{3} G_{i_{3}}\right)$ zugepaarten Punkte gehen, durch ein Tripel von $(p)^{3}$ gehen und also alle drei zugleich reell sein. Gleichzeitig folgt hieraus, daß $G_{i_{1}}, G_{i_{2}}, G_{i_{3}}$, die Berührungspunkte von $t_{i_{1}}, t_{i_{2}}, t_{i_{3}}$, ein reelles Tripel in $\left(p^{2}\right)^{3}$ bilden müssen und mithin $g_{i_{1}}, g_{i_{2}}, g_{i_{3}}$ ein reelles Strahlentripel in $(P)^{3}$. Nunmehr geht aber (nach Satz 30 und Nr.72) durch vier Punkte $P_{1}, P_{2}, P_{3}, P_{x}$ von $p^{3}$ dann und nur dann ein $p^{3}$ in $P_{x}$ dreipunktig berührender Kegelschnitt, wenn die Tangente $t_{x}$ von $p^{2}$ in $G_{x}$ durch den in $(i)^{2}$ zum Schnittpunkte $\left(p, G_{3} G_{x}\right)$ zugepaarten Punkt geht, wenn also $t_{x}$ eine der drei Tangenten $t_{i_{1}}, t_{i_{2}}, t_{i_{3}}$ ist und mithin $G_{x}$ einer der drei Berührungspunkte $G_{i_{1}}, G_{i_{2}}, G_{i_{3}}$.

Diese Lösung behält ihre Gültigkeit auch dann noch, wenn zwei der drei gegebenen Punkte oder alle drei aufeinander folgen; doch gibt es im letztern Falle, wie wir bald sehen werden, eine einfachere Lösung.

Die Ordinatenwinkel der drei gefundenen Punkte $P_{i_{1}}, P_{i_{2}}$, $P_{i_{3}}$ sind nach Satz 32 (für $k=0,1,2$ ):

$$
\begin{gathered}
\omega_{i_{1}}=-\frac{\omega_{1}+\omega_{2}+\omega_{3}}{3}, \quad \omega_{i_{2}}=\frac{\pi-\left(\omega_{1}+\omega_{2}+\omega_{3}\right)}{3}, \\
\omega_{i_{3}}=\frac{2 \pi-\left(\omega_{1}+\omega_{2}+\omega_{3}\right)}{3} .
\end{gathered}
$$

Dies bestätigt abermals, daß $g_{i_{1}} g_{i_{2}} g_{i_{3}}$ ein Tripel in $(P)^{3}$ bilden.

Aufgabe 19. Ein beliebiger Punkt $P_{1}$ von $p^{3}$ sei gegeben, es soll ein solcher zweiter Punkt auf $p^{3}$ gefunden werden; der zugleich mit $P_{1}$ je ein dreipunktiger Berührungspunkt von $p^{3}$ mit einem und demselben Kegelschnitt sein soll.

Auflösung. Man ermittelt den $\operatorname{Pol} G_{1}$ von $g_{1}$, verbindet diesen mit den drei Wendepunkten von $p^{3}$, also mit den drei Schnittpunkten $P_{a}, P_{b}, P_{c}$ von $p$ mit den Seiten von $A B C$, und ermittelt die zweiten Schnittpunkte $G_{i_{1}}, G_{i_{2}}, G_{i_{3}}$ der drei Geraden $G_{1} P_{a}, G_{1} P_{b}, G_{1} P_{c}$ mit $p^{2}$ und deren durch 
$P$ gehende Polaren (in bezug auf $A B C$ ) $g_{i_{1}}, g_{i_{2}}, g_{i_{3}}$; alsdann wird jeder der drei Punkte $P_{i_{1}}, P_{i_{2}}, P_{i_{3}}$ und nur einer dieser drei, welche in $\left(g_{i_{1}}\right)^{2},\left(g_{i_{2}}\right)^{2},\left(g_{i_{3}}\right)^{2}$ zu $P$ zugepaart sind, der in der Aufgabe gestellten Forderung genügen.

Die Ordinatenwinkel dieser drei Punkte sind nach Satz 32:

$$
\omega_{i_{1}}=-\omega_{1}, \quad \omega_{i_{2}}=\frac{\pi}{3}-\omega_{1}, \quad \omega_{i_{3}}=\frac{2 \pi}{3}-\omega_{1} .
$$

Diese Lösung ergibt sich in folgender Weise: Nach Satz 30 und Nr. 72 geht durch zwei Punkte $P_{1}$ und $P_{x}$ von $p^{3}$ dann und nur dann ein $p^{3}$ in jedem derselben dreipunktig berührender Kegelschnitt, wenn $g_{1}$ und $g_{x}$ durch ein Punktepaar derjenigen auf $(p)^{2}$ sich stützenden Involution gehen, deren einer Doppelpunkt der Schnittpunkt $Q_{y}$ von $p$ mit $G_{1} G_{x}$ ist, wenn also $g_{1} g_{x}$ ein Strahlenpaar in derjenigen auf $(P)^{2}$ sich stützenden Involution $(I)^{2}$ bilden, die $(i)^{2}$ von $P$ aus projiziert und deren einer Doppelstrahl also $g_{y}$, der $Q_{y}$ mit $P$ verbindende Strahl, ist. Dies ist aber (nach Nr.63) dann und nur dann der Fall, wenn $P_{y}$, der (nach II in Nr. 55) von $P$ aus durch $g_{y}$ projizierte dritte Schnittpunkt von $p^{3}$ mit $P_{1} P_{x}$, einerseits, weil $g_{1} g_{x}$ ein Paar in der auf $(P)^{2}$ sich stïtzenden Strahleninvolution $(I)^{2}$ bilden, derjenige Punkt ist, mit dem alle die durch Strahlenpaare von $(I)^{2}$ projizierten Punktepaare auf $p^{3}$ in je einer Geraden liegen, andererseits, weil $g_{y}$ ein Doppelstrahl von $(I)^{2}$ ist, ein solcher Punkt, dessen Tangentialpunkt derjenige letzt erwähnte Punkt und nunmehr also $P_{y}$ selbst ist; mithin wenn $P_{y}$ einer der drei Wendepunkte $P_{a}, P_{b}, P_{c}$ von $p^{3}$ ist und also $P_{x}$ einer der drei dritten Schnittpunkte von $p^{3}$ mit $P_{1} P_{a}, P_{1} P_{b}, P_{1} P_{c}$, welche drei Schnittpunkte aber (nach II) keine anderen als die ermittelten Punkte $P_{i_{1}}, P_{i_{2}}, P_{i_{3}}$ sind.

Aufgabe 20. Zwei beliebige Punkte $P_{1}$ und $P_{2}$ vom $p^{3}$ seien gegeben, es soll ein solcher Punkt auf $p^{3}$ gefunden werden, welcher ein vierpunktiger Berührungspunkt eines durch $P_{1}$ und $P_{2}$ gehenden Kegelschnitts mit $p^{3}$ sein soll.

Auflösung. Man ermittelt die beiden Doppelpunkte derjenigen auf $(p)^{2}$ sich stützenden Involution $(i)^{2}$, in der $Q_{1} Q_{2}$ $\left(Q_{1} \equiv p g_{1}, Q_{2} \equiv p g_{2}\right)$ ein Punktepaar bilden, sodann die vier Schnittpunkte $G_{k_{1}}, G_{k_{2}}, G_{k_{3}}, G_{k_{4}}$ von $p^{2}$ mit den die beïden ermittelten Doppelpunkte von $P$ aus projizierenden Gerauden und deren durch $P$ gehenden Polaren (in bezug auf $A B C$ ) 
$g_{k_{1}}, g_{k_{2}}, g_{k_{3}}, g_{k_{4}}$, welche letzte ein Strahlenquadrupel in der von $A B C$ um $P$ erzeugten Involution $(P)^{4}$ bilden, und also, wenn deren einer bekannt ist, die drei übrigen mit bestimmt sind; alsdann wird jeder der vier Punkte $P_{k_{1}}, P_{k_{2}}, P_{k_{3}}, P_{k_{4}}$ und nur einer dieser vier, welche in den von $A B C$ erzeugten Involutionen $\left(g_{k_{1}}\right)^{2},\left(g_{k_{2}}\right)^{2},\left(g_{k_{3}}\right)^{2},\left(g_{k_{4}}\right)^{2}$ zu $P$ zugepaart sind, der in der Aufgabe gestellten Forderung genügen.

Die Ordinatenwinkel von $P_{k_{1}}, P_{k_{2}}, P_{k_{3}}, P_{k_{4}}$ sind nach Satz 32 (für $k=0,1,2,3$ ):

$$
\begin{array}{ll}
\omega_{k_{1}}=-\frac{\omega_{1}+\omega_{2}}{4}, & \omega_{k_{2}}=\frac{\pi-\left(\omega_{1}+\omega_{2}\right)}{4}, \\
\omega_{k_{3}}=\frac{2 \pi-\left(\omega_{1}+\omega_{2}\right)}{4}, & \omega_{k_{4}}=\frac{3 \pi-\left(\omega_{1}+\omega_{2}\right)}{4} .
\end{array}
$$

Denn nach Satz 30 und Nr. 72 geht durch drei Punkte $P_{1}$ $P_{2}, P_{x}$ von $p^{3}$ dann und nur dann ein $p^{3}$ in $P_{x}$ vierpunktig berührender Kegelschnitt, wenn die Tangente $t_{x}$ von $p^{2}$ in $G_{x}$ durch einen Doppelpunkt von $(i)^{2}$ geht; dies ist aber, weil in bezug auf $p^{2}$ die Involution konjugierter Punkte auf $p$ mit $(p)^{2}$ identisch ist und $P$ der Pol von $p$ (Nr.2) und $(i)^{2}$ sich auf $(p)^{2}$ stützt und die Doppelpunkte von $(i)^{2}$ also ein Punktepaar in $(p)^{2}$ bilden, dann und nur dann der Fall, wenn $G_{x}$ einer der vier Punkte $G_{k_{1}}, G_{k_{2}}$, $G_{k_{3}}, G_{k_{4}}$ und mithin $P_{x}$ einer der vier Punkte $P_{k_{1}}, P_{k_{2}}, P_{k_{3}}, P_{k_{4}}$ ist. Nunmehr werden $G_{k_{1}}, G_{k_{2}}, G_{k_{3}}, G_{k_{4}}$ von $P$ aus durch diejenigen zwei Geraden projiziert, welche das aus den Doppelpunkten von $(i)^{2}$ bestehende Punktepaar von $(p)^{2}$ mit $P$ verbinden, welche zwei Gerade also, weil $(P)^{2}$ zu $(p)^{2}$ perspektiv ist, ein Strahlenpaar in $(P)^{2}$ bilden; mithin müssen (nach Satz 12 in Nr.22) $g_{k_{1}}, g_{k_{2}}, g_{k_{3}}, g_{k_{4}}$ ein Strahlenquadrupel in $(P)^{4}$ bilden.

Die gestellte Aufgabe hat also im allgemeinen vier eigentliche Lösungen, nur wenn die gegebenen Punkte $P_{1}$ und $P_{2}$ zwei aufeinander folgende sind, hat die Aufgabe nur zwei eigentliche Lösungen.

Denn wenn $P_{1}$ ein einfacher Berührungspunkt des verlangten Kegelschnitts sein soll, so wird $Q_{1}$ der eine Doppelpunkt von $(i)^{2}$ sein und die Berührungspunkte der beiden aus $Q_{1}$ an $p^{2}$ gehenden Tangenten werden (nach II in Nr. 55) diejenigen beiden Punkte auf $p^{3}$ liefern, deren Tangentialpunkt $P_{1}$ ist, und die hierdurch 
gewonnenen Kegelschnitte werden offenbar ausgeartete sein. Hiernach wird dieser Fall wie folgt gelöst.

Aufgabe 21. Ein Punkt $P_{1}$ von $p^{3}$ sei gegeben, es soll ein solcher Punkt auf $p^{3}$ gefunden werden, welcher ein vierpunktiger Berührungspunkt eines $p^{3}$ in $P_{1}$ einfach berührenden Kegelschnitts sein soll.

Auflösung. Man ermittelt die beiden Schnittpunkte $G_{k_{1}}$ und $G_{k_{2}}$ von $g_{1}$ mit $p^{2}$ und deren durch $P$ gehende Polaren $g_{k_{1}}$ und $g_{k_{2}}$, welche letzte dasjenige Strahlenpaar in $(P)^{2}$ bilden, dessen Repräsentant $g_{1}$ ist; alsdann wird jeder der beiden Punkte $P_{k_{1}}$ und $P_{k_{2}}$, welche in $\left(g_{k_{1}}\right)^{2}$ und $\left(g_{k_{2}}\right)^{2} \mathrm{zu}$ $P$ zugepaart sind, von der verlangten Art sein.

Die Ordinatenwinkel von $P_{k_{1}}$ und $P_{k_{2}}$ sind:

$$
\omega_{k_{1}}=-\omega_{1}: 2, \quad \omega_{k_{2}}=\left(\pi-\omega_{1}\right): 2 .
$$

Diese Aufgabe und Auflösung sind die Umkehrungen zu der Aufgabe 15 und deren Auflösung.

\section{Aus Satz 32 folgt ferner:}

Ist ein Punkt $P_{1}$ von $p^{3}$ gegeben, so gibt es auf $p^{3}$ fünf und nur fünf Punkte $P_{l_{1}}, P_{l_{2}}, P_{l_{3}}, P_{l_{4}}, P_{l_{5}}$, deren jeder ein fünfpunktiger Berührungspunkt eines durch $P_{1}$ gehenden Kegelschnitts mit $p^{3}$ ist. Die Ordinatenwinkel dieser fünf Punkte sind:

$$
\begin{gathered}
\omega_{l_{1}}=-\frac{\omega_{1}}{5}, \quad \omega_{l_{2}}=\frac{\pi-\omega_{1}}{5}, \quad \omega_{l_{3}}=\frac{2 \pi-\omega_{1}}{5}, \\
\omega_{l_{4}}=\frac{3 \pi-\omega_{1}}{5}, \quad \omega_{l_{5}}=\frac{4 \pi-\omega_{1}}{5} .
\end{gathered}
$$

Aus dem Satze 30 ergibt sich noch:

Die Ecken von $A B C$ sind die einzigen drei Punkte auf $p^{3}$, in deren jedem $p^{3}$ von einem eigentlichen Kegelschnitt sechspunktig berührt wird.

Denn $p^{3}$ wird (nach Satz 30 und Nr. 72) dann und nur dann in einem Punkte $P_{i}$ von einem Kegelschnitt sechspunktig berührt, wenn die Tangente $t_{i}$ von $p^{2}$ in $G_{i}$ durch einen Doppelpunkt derjenigen auf $(p)^{2}$ sich stützenden Involution geht, in der $Q_{i} \equiv p g_{i}$ einer der Doppelpunkte ist. Der verlangte Kegelschnitt ist aber (weil, wenn $t_{i}$ durch $Q_{i}$ geht und mithin $P_{i}$, der dann nach II (Nr.55) 
mit seinem Tangentialpunkt zusammenfallen muß, ein Wendepunkt von $p^{3}$ ist, der verlangte Kegelschnitt in die doppelt zu zählende Wendetangente ausartet) nur dann ein eigentlicher, wenn $Q_{i}$ und $p t_{i}$ die beiden Doppelpunkte einer auf $(p)^{2}$ sich stützenden Involution sind und also ein Punktepaar in $(p)^{2}$ bilden; dies ist aber nur dann der Fall, wenn die $P_{i}$ und seinen Tangentialpunkt von $P$ aus projizierenden Geraden $P Q_{i}$ und $\left(P, p t_{i}\right)$ ein Strahlenpaar in $(P)^{2}$ bilden, also, weil zwei durch ein Strahlenpaar von $(P)^{2}$ projizierte Punkte von $p^{3}$ einen und denselben Tangentialpunkt haben (nach I in Nr.54), wenn $P_{i}$ mit einem der Wendepunkte von $p^{3}$ durch ein Strahlenpaar von $(P)^{2}$ projiziert wird, und mithin, weil die Wendepunkte $P_{a}, P_{b}, P_{c}$ von $P$ aus durch die in $(P)^{2}$ zu $p_{A} \equiv P A, p_{B} \equiv P B, p_{C} \equiv P C$ zugepaarten Strahlen $p_{A}^{\prime}, p_{B}^{\prime}, p_{C}^{\prime}$ projiziert werden (Nr.5), nur dann, wenn $P_{i}$ eine der drei Ecken von $A B C$ ist. Dasselbe kann auch mit Hilfe der Ordinatenwinkel nachgewiesen werden.

Wir wollen hier noch die Lösung der folgenden Aufgabe angeben.

A ufgabe 22. Vier beliebige Punkte $P_{1}, P_{2}, P_{3}, P_{4}$ von $p^{3}$ seien gegeben, es soll ihr Gegenpunkt (Nr. 73) gefunden werden.

Auflösung. Man ermittelt etwa die Pole $G_{1}$ und $G_{2}$ von $g_{1}$ und $g_{2}$, verbindet etwa $G_{1}$ mit $Q_{3} \equiv p g_{3}$ und $G_{2}$ mit $Q_{4} \equiv p g_{4}$ und bestimmt die zweiten Schnittpunkte $G_{5}$ und $G_{6}$ von $p^{2}$ mit $Q_{3} G_{1}$ und $Q_{4} G_{2}$ und sodann den Schnittpunkt $Q_{7}$ von $p$ mit $G_{5} G_{6}$; alsdann wird derjenige Punkt $P_{7}$, welcher in der von $A B C$ auf $g_{7} \equiv P Q_{7}$ erzeugten Involution $\left(g_{7}\right)^{2}$ zu $P$ zugepaart ist, der gesuchte Gegenpunkt sein.

Denn nach Nr. 73 müssen auch die zwei weiteren Schnittpunkte $P_{5}$ und $P_{6}$ von $p^{3}$ mit dem in das Geradenpaar $P_{1} P_{3}$ und $P_{2} P_{4}$ ausgearteten Kegelschnitt mit dem Gegenpunkte von $P_{1} P_{2} P_{3} P_{4}$ in einer Geraden liegen. Nun sind nach II (Nr. 55) $G_{5} \equiv\left(p^{2}, Q_{1} G_{3}\right)$ und $G_{6} \equiv\left(p^{2}, Q_{2} G_{4}\right)$ die Pole der $P_{5}$ und $P_{6}$ von $P$ aus projizierenden Geraden $g_{5}$ und $g_{6}$, mithin muß (ebenfalls nach II) der Gegenpunkt, der dritte Schnittpunkt von $p^{3}$ mit $P_{5} P_{6}$, von $P$ aus durch $g_{7} \equiv P Q_{7}\left(Q_{7} \equiv p G_{5} G_{6}\right)$ projiziert werden und also $P_{7}$ sein.

Der Ordinatenwinkel des Gegenpunktes $P_{7}$ ist:

$$
\omega_{7}=\frac{2 k+1}{2} \pi+\omega_{1}+\omega_{2}+\omega_{3}+\omega_{4} .
$$


Denn nach Satz 24 ist:

also

$$
\begin{aligned}
& \omega_{7}=\frac{2 k_{1}+1}{2} \pi-\omega_{5}-\omega_{6}, \\
& \omega_{5}=\frac{2 k_{2}+1}{2} \pi-\omega_{1}-\omega_{3}, \\
& \omega_{6}=\frac{2 k_{3}+1}{2} \pi-\omega_{2}-\omega_{4},
\end{aligned}
$$

$$
\begin{aligned}
\omega_{7} & =\left(k_{1}-k_{2}-k_{3}\right) \pi-\frac{\pi}{2}+\omega_{1}+\omega_{2}+\omega_{3}+\omega_{4} \\
& =\frac{2 k+1}{2} \pi+\omega_{1}+\omega_{2}+\omega_{3}+\omega_{4} .
\end{aligned}
$$

Die gegebene Lösung behält ihre Gültigkeit auch dann noch bei, wenn mehrere der vier gegebenen Punkte oder sogar alle vier aufeinander folgen; nur muß alsdann das in $\mathrm{Nr} .72$ Behauptete berücksichtigt werden. Hiernach haben wir, wenn vier aufeinanderfolgende Punkte von $p^{3}$ in $P_{1}$ vereinigt liegen, im zweiten Schnittpunkte von $p^{2}$ mit $Q_{1} G_{1}$, also nach II im Pole der den ersten Tangentialpunkt von $P_{1}$ aus $P$ projizierenden Geraden, die Tangente an $p^{2}$ zu legen, diese mit $p$ zum Schnitt zu bringen und diesen Schnittpunkt mit $P$ durch eine Gerade zu verbinden; alsdann wird diese Gerade den Gegenpunkt der vier in $P_{1}$ vereinigt liegenden Punkte enthalten, welcher Gegenpunkt nach II der Tangentialpunkt des ersten Tangentialpunktes von $P_{1}$, also der zweite Tangentialpunkt von $P_{1}$ ist.

Alle in diesem und im vorhergehenden Paragraphen gefundenen Resultate und Konstruktionen gelten, dualisiert, für das zu $p^{3}$ duale Gebilde $P^{3}$.

\section{\$11.}

77. Bisher haben wir (Nr. 48, 59) nur bewiesen, daß keine Gerade mehr als drei Punkte mit $p^{3}$ gemein hat, und daß, wenn eine Gerade einen Punkt mit $p^{3}$ gemein hat, dieselbe noch zwei Punkte (reelle oder imaginäre) mit $p^{3}$ gemein haben muß. Ob aber jede Gerade mit $p^{3}$ einen und mithin drei Punkte gemein haben muß, blieb dahin gestellt. Diese Frage wird aber durch die folgende Überlegung leicht erledigt.

Nach Definition (Nr. 46) wird $p^{3}$ von dem in der von $A B C$ auf einem Strahle $g_{x}$ von $P$ erzeugten Punktinvolution $\left(g_{x}\right)^{2} \mathrm{zu}$ 
$P$ zugepaarten Punkte $P_{x}$ beschrieben, wenn $g_{x}$ den ganzen Strahlenbüschel um $P$ stetig durchläuft. Nun ist auf $g_{x}$ in jeder seiner Lagen nur ein einziger solcher Punkt $P_{x}$ vorhanden, und die Punktinvolution $\left(g_{x}\right)^{2}$ wird durch die Schnittpunkte von $g_{x}$ mit den drei Seiten von $A B C$ in der oben (Nr. 4) angegebenen Weise festgelegt, welche Schnittpunkte gleichzeitig mit $g_{x}$ stetig aufeinander folgen. Mithin muß $p^{3}$ aus einem einzigen stetigen und geschlossenen Linienzuge bestehen (abgesehen natürlich von dem isolierten Doppelpunkte $P$ ).

Nehmen wir nun irgend zwei reelle Punkte von $p^{3}$, etwa die Eckpunkte $A$ und $B$, und irgendeine durch keinen dieser Punkte gehende reelle Gerade, von der wir wissen, daß sie mit $p^{3}$ drei reelle Punkte gemein hat, etwa die Polare $p$ von $P$, so muß $p$, wenn die ganze Kurve $p^{3}$ von $A$ aus in derjenigen Richtung einmal durchlaufen wird, in $\operatorname{der}$ nach $B$ gelangt wird bei der kleinst möglichen Anzahl von Überschreitungen durch $p$, entweder auf dem Hinwege von $A$ nach $B$ keinmal und auf dem Rückwege von $B$ nach $A$ dreimal überschritten werden, oder auf dem Hinwege einmal und auf dem Rückwege zweimal. In beiden Fällen kann man also von $A$ nach $B$ (der Kurve $p^{3}$ entlang) auf zwei Weisen gelangen: durch eine gerade Anzahl (0 oder 2) von Überschreitungen durch $p$ und durch eine ungerade Anzahl (3 oder 1).

Nun wird die ganze Ebene durch $p$ und irgendeine beliebige reelle Gerade $l$ in zwei Gebiete geteilt, nämlich in die zwei Paar von $p$ und $l$ gebildeten Scheitelwinkel; und es führt dann eine ungerade Anzahl von Überschreitungen durch $p$ und $l$ von dem einen Gebiete in das zweite über, dagegen eine gerade Anzahl in das Ausgangsgebiet zurück; von einem Punkte des einen Gebietes kann man also zu einem Punkte desselben Gebietes nur durch eine gerade Anzahl von Überschreitungen durch $p$ und $l$ gelangen, dagegen zu einem Punkte des zweiten Gebietes nur durch eine ungerade Anzahl.

Geht nun $l$ durch $A$ oder $B$, so hat sie mit $p^{3}$ bereits diesen Punkt und mithin noch zwei gemein. Geht ferner $l$ weder durch $A$ noch durch $B$, so können dann nur zwei Fälle eintreten: erstens, $A$ und $B$ gehören einem und demselben der beiden durch $p$ und $l$ getrennten Ebenegebiete an, zweitens, $A$ und $B$ gehören verschiedenen dieser beiden Ebenegebiete an. Gehen wir nun im ersten Falle, wo $A$ und $B$ demselben Gebiete angehören, von $A$ nach $B$ 
der Kurve $p^{3}$ entlang auf demjenigen Wege, auf dem $p$ ungeradzählig überschritten wird, so muß auf diesem Wege, weil nur eine gerade Anzahl von Überschreitungen durch $p$ und $l$ in das Ausgangsgebiet zurückführt, notwendigerweise auch $l$ ungeradzählig, also mindestens einmal überschritten werden; im zweiten Falle, wo $A$ und $B$ verschiedenen Gebieten angehören, gehen wir von $A$ nach $B$ der Kurve $p^{3}$ entlang auf demjenigen Wege, auf dem $p$ geradzählig überschritten wird; alsdann muß auf diesem Wege, weil nur eine ungerade Anzahl von Utberschreitungen durch $p$ und $l$ von dem einen Gebiete in das zweite überführt, $l$ ungeradzählig, also mindestens einmal überschritten werden. Wir sehen also, daß jede beliebige reelle Gerade $l$ mit $p^{3}$ mindestens einen reellen Punkt, nämlich den angegebenen Überschreitungspunkt, und mithin noch zwei Punkte, die auch konjugiert-imaginär sein können, gemein hat.

78. Nunmehr können wir (nach Nr. 46, 59, 60) den folgenden Satz aufstellen.

Satz 33. Der Ort $p^{3}$ derjenigen Punkte, welche einem auf keiner Seite von $A B C$ liegenden reellen Punkte $P$ zugepaart sind in den von $A B C$ auf den sämtlichen durch $P$ gehenden Geraden $g$ erzeugten Punktinvolutionen $(g)^{2}$, welche Punkte also dem $P$ konjugiert sind in bezug auf $A B C$, ist eine Kurve dritter Ordnung, von der $P$ ein isolierter Doppelpunkt ist und die beiden konjugiertimaginären Doppelstrahlen der von $A B C$ erzeugten Strahleninvolution $(P)^{2}$ die Doppelpunktstangenten sind. Die Kurve $p^{3}$ ist $A B C$ umschrieben und hat in dessen
Das aus denjenigen Strahlen bestehende Gebilde $P^{3}$, welche einer durch keine Ecke von $A B C$ gehenden reellen Geraden $p$ zugepaart sind in den von $A B C$ um die sämtlichen auf $p$ liegenden Punkte $Q$ erzeugten Strahleninvolutionen $(Q)^{2}$, welche Strahlen also der $p$ konjugiert sind in bezug auf $A B C$, ist ein Strahlenbüschel dritter Ordnung, umhüllt also eine Kurve dritter Klasse, von der $p$ eine isolierte Doppeltangente ist und die beiden konjugiert-imaginären Doppelpunkte der von $A B C$ erzeugten Punktinvolution $(p)^{2}$ die Doppeltangentenberüh- 
Ecken dieselben Tangenten wie der Polarkegelschnitt $p^{2}$ von $P$, nämlich die drei vierten harmonischen Geraden $A P_{a}, B P_{b}, C P_{c}$ zu bzw. $A P \equiv p_{A}, \quad B P \equiv p_{B}$, $C P \equiv p_{C}$ in bezug auf je zwei Seiten von $A B C$; diese drei Tangenten von $p^{3}$ in den Ecken von $A B C$ treffen die Gegenseiten in den Wendepunkten $P_{a}, P_{b}, P_{c}$ von $p^{3}$, welche drei Punkte auf der Polare (in bezug auf $A B C) p$ von $P$ liegen. $P$ und $p$ sind also Zentrum und Achse der beiden perspektiven Dreiecke, welche von den drei aus den Wendepunkten an $p^{3}$ gehenden Tangenten und deren Berührungspunkte gebildet werden.

Kein Punkt von $p^{3}$ liegt im Innern des Polarkegelschnitts $p^{2}$ von $P$ und umsomehr im Innern desjenigen Dreiecksgebietes von $A B C$, innerhalb dessen $P$ liegt und welches Gebiet also (Nr. 2) ganz dem Innern von $p^{2}$ angehört.

Wir wollen $p^{3}$ die zu $P$ in bezug auf $A B C \mathrm{zu}$ - rungspunkte sind. Die von $P^{3}$ eingehüllte Kurve ist $A B C$ eingeschrieben und ihre Berührungspunkte mit dessen Seiten sind dieselben wie die von dem Polarkegelschnitt $P^{2}$ von $p$, nämlich die drei vierten harmonischen Punkte $a p_{A}, b p_{B}$, $c p_{C}$ zu bzw. $a p \equiv P_{a}, b p \equiv P_{b}$, $c p \equiv P_{c}$ in bezug auf je zwei Ecken von $A B C$; diese drei Berührungspunkte von $P^{3}$ in den Seiten von $A B C$ mit den Gegenecken verbunden liefern die drei Rückkehrtangenten $p_{A}, p_{B}, p_{C}$ von $P^{3}$, welche letztere durch den Pol (in bezug auf $A B C$ ) $P$ von $p$ gehen. $p$ und $P$ sind also Achse und Zentrum der beiden perspektiven Dreiecke, welche von den drei auf den Rückkehrtangenten liegenden Berührungspunkten und deren Tangenten gebildet werden.

Jeder Strahl (Tangente) von $P^{3}$ schneidet den Polarkegelschnitt $P^{2}$ von $p$ in zwei reellen Punkten und muß also in dasjenige Dreiecksgebiet von $A B C$ eindringen, innerhalb dessen $P$ und mithin (Nr. 2) der ganze Polarkegelschnitt $P^{2}$ liegt.

Wir wollen $P^{3}$ den zu $p$ in bezug auf $A B C$ zuge- 
gehörige Kurve dritter Ordnung nennen.

hörigen Strahlenbüschel dritter Ordnung nennen.

Ist die Gerade $p$ rechts die Polare (in bezug auf $A B C$ ) des Punktes $P$ links, so besteht der Büschel $P^{3}$ rechts aus den Polaren (in bezug auf $A B C$ ) der sämtlichen Punkte der Kurve $p^{3}$ links; und zwar ist ein Strahl $p_{i}$ von $P^{3}$ die Polare desjenigen Punktes $P_{i}$ von $p^{3}$, welcher durch den $P$ mit $Q_{i}$, dem Schnittpunkte von $p$ mit $p_{i}$, verbindenden Strahl $g_{i}$ von $P$ aus projiziert wird.

Daß kein Punkt von $p^{3}$ innerhalb $p^{2}$ liegt, erkennt man in folgender Weise: Die Schnittpunkte von $p^{2}$ mit einem jeden Strahle $g_{i}$ von $P$ bilden, weil $P$ das Zentrum der durch $(P)^{2}$ auf $p^{2}$ induzierten krummen Punktinvolution $\left(p^{2}\right)^{2}$ ist (Nr. 7), ein Punktepaar in $\left(p^{2}\right)^{2}$ und mithin nach Satz 1 (Nr.7), weil ein Punktepaar von $\left(p^{2}\right)^{2}$ aus den Polen eines Strahlenpaares von $(P)^{2}$ besteht; auch ein Punktepaar in der von $A B C$ auf $g_{i}$ erzeugten Punktinvolution $\left(g_{i}\right)^{2}$. Nun ist $\left(g_{i}\right)^{2}$ wie alle von $A B C$ erzeugten Involutionen elliptisch (Nr.4) und zwei Paare einer elliptischen Involution werden stets durcheinander getrennt; mithin muß der auf $g_{i}$ liegende Punkt $P_{i}$ von $p^{3}$, der in $\left(g_{i}\right)^{2}$ zu $P$ zugepaarte Punkt, auf derjenigen der beiden durch die Schnittpunkte von $g_{i}$ mit $p^{2}$ begrenzten Strecken von $g_{i}$ liegen, welche außerhalb $p^{2}$ verläuft, da $P$ auf der innerhalb $p^{2}$ verlaufenden Strecke von $g_{i}$ liegt (nach Nr. 2).

Daß ferner die Strahlen von $P^{3}$ aus den Polaren der Punkte von $p^{3}$ bestehen, ergibt sich wie folgt: Nach Satz 1 muß der Pol eines Strahles $p_{i}$ von $P^{3}$, weil (nach Definition von $P^{3}$ ) $p_{i}$ zu $p$ in $\left(Q_{i}\right)^{2}$ zugepaart ist, mit dem Pole $P$ von $p$ in einer durch $Q_{i}$ gehenden Geraden liegen, also in $g_{i} \equiv P Q_{i}$; und zwar muß dieser Pol derjenige Punkt von $g_{i}$ sein, welcher zu $P$ in $\left(g_{i}\right)^{2}$ zugepaart ist, mithin der von $P$ aus durch $g_{i}$ projizierte Punkt $P_{i}$ von $p^{3}$.

79. Die Tatsache, die wir soeben erkannten, nämlich, daß die Polare $p_{i}$ eines von $P$ aus durch den Strahl $g_{i}$ projizierten Punktes $P_{i}$ von $p^{3}$ durch den Schnittpunkt $Q_{i}$ von $g_{i}$ mit $p$ gehen muß und umgekehrt, wenn die Polare $p_{i}$ eines Punktes $P_{i}$ von $g_{i}$ durch $Q_{i} \equiv g p_{i}$ geht, $P_{i}$ nach Satz 1 in $\left(g_{i}\right)^{2}$ zu $P$ zugepaart sein $\mathrm{muß}$ und mithin ein Punkt von $p^{3}$, liefert nun einen zweiten Beweis dafür, daß jede beliebige reelle oder imaginäre Gerade $l$ 
mit $p^{3}$ drei Punkte und nur drei gemein hat, und gestattet zugleich die Aufgabe: die gemeinschaftlichen Punkte einer beliebigen Geraden mit $p^{3}$ zu bestimmen, auf die Ermittlung der drei gemeinschaftlichen Tangenten zweier Kegelschnitte, von denen die vierte gemeinschaftliche Tangente bekannt ist, zurückzuführen.

Ist nämlich eine beliebige Gerade $l$ gegeben und ist die Punktreihe $l(R)$ die Spur des Strahlenbüschels $P(g)$ auf $l$, so ist die Punktreihe $p(Q)$, die Spur von $P(g)$ auf der Polare $p$ von $P$, zu $l(R)$ perspektiv und mithin nach Nr. 2 zu dem Büschel der Polaren $L^{2}(r)$, dem Büschel der Tangenten um den Polarkegelschnitt $L^{2}$ von $l$, projektiv, wobei einer Tangente $r_{i}$ von $L^{2}$ derjenige Punkt $Q_{i}$ auf $p$ entspricht, der mit dem Pole $R_{i}$ von $r_{i}$ in einem durch $Y$ gehenden Strahle, also in $g_{i} \equiv P R_{i}$ liegt; und es gehen dann bekanntlich drei und nur drei Tangenten von $L^{2}$ durch die entsprechenden Punkte der projektiven Punktreihe $p(Q)$, von welchen drei Tangenten, wenn $l$ und mithin auch $L^{2}$ reell ist, mindestens eine reell ist, die beiden andern aber auch konjugiert-imaginär sein können. Nun liegt, wie wir sahen, ein Punkt $R_{i} \equiv l g_{i}$ dann und nur dann auf $p^{3}$, wenn die Polare $r_{i}$ von $R_{i}$ durch $Q_{i} \equiv p g_{i}$ geht, wenn also $r_{i}$, die Tangente an $L^{2}$, durch den entsprechenden Punkt $Q_{i}$ von $p(Q)$ geht. Mithin hat jede beliebige Gerade $l$ mit $p^{3}$ drei und nur drei Punkte gemein, von denen, wenn $l$ reell ist, mindestens einer reell ist, die beiden andern auch konjugiert-imaginär sein können.

Zugleich haben wir die folgende Lösung der

Aufgabe 23. Die drei Schnittpunkte von $p^{3}$ mit einer gegebenen Geraden $l \mathrm{zu}$ bestimmen.

Auflösung. Man bestimmt die Schnittpunkte $Q_{x}$ und $Q_{y}$ von $p$, der Polare von $P$, mit den beiden Geraden $P L_{b}$ und $P L_{c}$, wo $L_{b} \equiv(l, C A)$ und $L_{c} \equiv(l, A B)$, verbindet $Q_{x}$ mit $C$ und $Q_{y}$ mit $B$ und ermittelt sodann die drei gemeinschaftlichen Tangenten des Polarkegelschnitts (in bezug auf $A B C$ ) $L^{2}$ von $l$ und desjenigen Kegelschnitts $x^{2}$, der von den vier Geraden $p, B C, B Q_{y}, C Q_{x}$ tangiert wird und den Schnittpunkt $(p, P A)$ zum Berührungspunkt in $p$ hat (wo eine gemeinschaftliche Tangente von $L^{2}$ und $x^{2}$ bereits bekannt ist, nämlich die Dreieckseite $B C$ ); alsdann werden 
die Pole (in bezug auf $A B C$ ) dieser drei gemeinschaftlichen Tangenten die gesuchten Schnittpunkte von $p^{3}$ mit $l$ sein.

Denn der Büschel der Tangenten $L^{2}(r)$, der zu $p(Q)$ projektiv ist, schneidet $B C$, die als Seite von $A B C$ Tangente eines jeden Polarkegelschnitts einer Geraden ist, in eine zu $p(Q)$ projektive Punktreihe. Diese beiden projektiven Punktreihen auf $p$ und $B C$ erzeugen nun einen Kegelschnitt, welcher von $p$ und $B C$ tangiert wird und ebenso von $Q_{x} C$ und $Q_{y} B$ (da $Q_{x}$ mit $L_{b}$ und $Q_{y}$ mit $L_{c}$ in je einer durch $P$ gehenden Geraden liegen und $C$ der Schnittpunkt von $C A$, der Polare von $L_{b} \equiv(l, C A)$, mit $B C$ ist und $B$ der Schnittpunkt von $A B$, der Polare von $L_{c} \equiv(l, A B)$, mit $B C$ und mithin $Q_{x} C$ und $Q_{y} B$ Verbindungsgeraden entsprechender Punkte der beiden projektiven, den Kegelschnitt erzeugenden Punktreihen sind) und in $p$ den Schnittpunkt $(p, P A)$ zum Berührungspunkt hat (da die Polare des Schnittpunktes von $l$ mit $P A$, ebenso wie die Polare $p$ von $P$, durch den vierten harmonischen Punkt zu $(P A, B C)$ in bezug auf $B$ und $C$, also durch $P_{a} \equiv(p, B C)$, gehen muß und mithin dem gemeinschaftlichen Punkte $P_{a}$ von $p$ und $B C$, als $B C$ angehörig, der Punkt $(p, P A)$ in $p$ entspricht) und folglich mit $x^{2}$ identisch sein muß. Nun liegt ein Punkt $R_{i} \equiv l g_{i}$ dann und nur dann auf $p^{3}$, wenn die Polare $r_{i}$ von $R_{i}$, die Tangente von $L^{2}$, durch den entsprechenden Punkt $Q_{i} \equiv p g_{i}$ der zu $L^{2}(r)$ projektiven Punktreihe $p(Q)$ geht, wenn also $r_{i}$ zwei entsprechende Punkte der beiden projektiven Punktreihen auf $p$ und $B C$ verbindet, mithin wenn $r_{i}$, die Tangente an $L^{2}$, zugleich Tangente an $x^{2}$ ist.

80. Aus dem Satze 33 ergibt sich nun:

Satz 34. Sind $P$ und $p$ Pol und Polare in bezug auf $A B C, p^{3}$ und $P^{3}$ die zu $P$ bzw. der zu $p$ in bezug auf $A B C$ zugehörige Kurve bzw. Strahlenbüschel dritter Ordnung, so ist

$$
p^{3}
$$$$
P^{3}
$$

die Enveloppe desjenigen ( $A B C$ umschriebenes bzw. eingeschriebenes) Kegelschnittsystems, welches aus den Polarkegelschnitten (in bezug auf $A B C$ )

der sämtlichen Punkte der von $P^{3}$ eingehüllten Kurve der sämtlichen Tangenten der Kurve $p^{3}$ besteht. dritter Klasse besteht. 
Denn jeder Punkt $P_{i}$ von $p^{3}$, der nach Satz 33 der Pol des Strahles $p_{i}$ von $P^{3}$ ist, ist der vierte Schnittpunkt zweier aufeinanderfolgender Kegelschnitte jenes ( $A B C$ umschriebenes) Systems, nämlich der vierte Schnittpunkt der Polarkegelschnitte derjenigen beiden aufeinanderfolgenden Punkte der von $P^{3}$ eingehüllten Kurve, in denen diese Kurve von $p_{i}$ berührt wird.

Ferner ergibt sich aus Satz 33:

Satz 35. Sind $P$ und $p$ Pol und Polare in bezug auf $A B C$, so bilden

die Tangente $t_{i}$ im Punkte $P_{i}$ von $p^{3}$ und die Verbindungsgerade von $P_{i}$ mit dem Berührungspunkte $\mathfrak{U}_{i}$ des Strahles $p_{i}$ von $P^{3}$ (wo $p_{i}$ die Polare von $P_{i}$ in bezug auf $A B C$ ist), also $\mathrm{t}_{i}$ und $P_{i} \mathfrak{U}_{i}$ ein Strahlenpaar in der von $A B C$ erzeugten Involution $\left(P_{i}\right)^{2}$. der Berührungspunkt $\mathfrak{U}_{i}$ des Strahles $p_{i}$ von $P^{3}$ und der Schnittpunkt von $p_{i}$ mit der Tangente $t_{i}$ im Punkte $P_{i}$ von $p^{3}$ (wo $P_{i}$ der Pol von $p_{i}$ in bezug auf $A B C$ ist), also $\mathfrak{H}_{i}$ und $p_{i} \mathrm{t}_{i}$ ein Punktepaar in der von $A B C$ erzeugten Involution $\left(p_{i}\right)^{2}$.

Denn die Tangente $\mathrm{t}_{i}$ von $p^{3}$ in $P_{i}$, die zwei aufeinanderfolgende Punkte von $p^{3}$, also nach Satz 33 zwei Pole der beiden aufeinanderfolgenden, im Berührungspunkte $\mathfrak{U}_{i}$ von $p_{i}$ sich schneidenden Strahlen von $P^{3}$ verbindet, ist zugleich die Tangente im Punkte $P_{i}$ des Polarkegelschnitts (in bezug auf $A B C$ ) $\mathfrak{u}_{i}^{2}$ von $\mathfrak{u}_{i}$ (was auch aus Satz 34 folgt); mithin müssen nach Hilfssatz 2 (Nr. 52) $\mathrm{t}_{i}$ und $P_{i} \mathfrak{U}_{i}$ ein Paar in $\left(P_{i}\right)^{2}$ bilden.

Zugleich haben wir die folgende Konstruktion für die Tangente in irgendeinem Punkte $P_{i}$ von $p^{3}$ gewonnen.

Auflösung 4 der Aufgabe 3 (Nr.51). Man ermittelt den Pol $G_{i}$ der Geraden $g_{i} \equiv P P_{i}$, sodann auf der Geraden $g_{i}^{(1)} \equiv P G_{i}$, dem ersten Repräsentanten von $g_{i}$, den Punkt $P_{i}^{(1)}$ von $p^{3}$, welcher Punkt $P_{i}^{(1)}$ zu $P$ zugepaart ist in der von $A B C^{\prime}$ erzeugten Punktinvolution $\left(g_{i}^{(1)}\right)^{2}$, und darauf die Tangente $\mathrm{t}_{i}$ im Punkte $P_{i}$ desjenigen Kegelschnitts, welcher durch die fünf Punkte $A, B, C, P_{i}$ und $P_{i}^{(1)}$ geht; alsdann wird diese Tangente zugleich die gesuchte Tangente von $p^{3}$ in $P_{i}$ sein. 
Diese Konstruktion ergibt sich folgendermaßen: Wie wir eben sahen, haben $p^{3}$ und $\mathfrak{u}_{i}^{2}$ eine gemeinsame Tangente in $P_{i}$, und $\mathfrak{u}_{i}^{2}$, der Polarkegelschnitt von $\mathfrak{u}_{i}$, ist $A B C$ umschrieben und geht durch $P_{i}$ und $P_{i}^{(1)}$; da durch den Berührungspunkt $\mathfrak{H}_{i}$ des Strahles $p_{i}$ von $P^{3}$ außer $p_{i}$ noch derjenige Strahl $p_{i}^{(1)}$ von $P^{3}$ geht, welcher von $p$ in dem zum Punkte $Q_{i}^{(1)}$, zum ersten Repräsentanten von $Q_{i} \equiv p p_{i}$, in der von $A B C$ erzeugten Punktinvolution $(p)^{2}$ zugepaarten Punkte geschnitten wird (dual der Aufl. 3 der Aufg. 5 in Nr.61), und durch diesen letzten Schnittpunkt der Strahl $g_{i}^{(1)}$, der erste Repräsentant von $g_{i} \equiv P P_{i} \equiv P Q_{i}$, geht (nach Satz 14 in Nr. 23) und mithin nach Satz $33 P_{i}^{(1)}$ der Pol von $p_{i}^{(1)}$ ist. Folglich brauchen wir nur die Tangente in $P_{i}$ desjenigen Kegelschnitts zu bestimmen, welcher durch die fünf Punkte $A, B, C, P_{i}, P_{i}^{(1)}$ geht und somit mit $\mathfrak{u}_{i}^{2}$ identisch ist.

81. Wir wollen nun zeigen, daß unsere Kurve $p^{3}$ und Strahlenbüschel $P^{3}$ auch Erzeugnisse je zweier gewisser projektiver Gebilde sind und mithin keine andere als eine der bekannten Kurven dritter Ordnung mit isoliertem Doppelpunkt bzw. einer der bekannten Strahlenbüschel dritter Ordnung mit isoliertem Doppelstrahl.

Nach Satz 24 (Nr.53) liegt nämlich der von $P$ aus durch den Strahl $g_{i}$ projizierte Punkt $P_{i}$ von $p^{3}$ zugleich auf $t_{i}$, der Tangente am Polarkegelschnitt $p^{2}$ von $P$ im Pole $G_{i}$ von $g_{i}$. Nun ist aber (Nr.2) der Strahlenbüschel $P(g)$ zu der krummen Punktreihe seiner Pole auf $p^{2}$, also zu $p^{2}(G)$, und mithin auch zu dem die letzte umhüllenden Büschel der Tangenten, also zu $p^{2}(t)$, projektiv. Somit haben wir:

Die Kurve $p^{3}$ ist das Erzeugnis zweier projektiver Büschel erster und zweiter Ordnung, nämlich des Strahlenbüschels $P(g)$ und des Büschels $p^{2}(t)$ derTangenten um den Polarkegelschnitt $p^{2}$ von $P$, wo einer Tangente $t_{i}$ von $p^{2}$, deren Berührungspunkt $G_{i}$ ist, die durch $P$ gehende Polare $g_{i}$ von $G_{i}$ entspricht.
Der Büschel $P^{3}$ ist das Erzeugnis zweier projektiver Punktreihen erster und zweiter Ordnung, nämlich der geraden Punktreihe $p(Q)$ und der krummen Punktreihe $P^{2}(U)$ auf dem Polarkegelschnitt $P^{2}$ von $p$, wo einem Punkte $U_{i}$ von $P^{2}$, dessen Tangente $q_{i}$ ist, der auf $p$ liegende Pol $Q_{i}$ von $q_{i}$ entspricht. 
Ferner ist $p^{3}$ auch das Erzeugnis desjenigen Kegelschnittbüschels, welcher aus den Polarkegelschnitten $q^{2}$ der sämtlichen auf der Polare $p$ von $P$ liegenden Punkte $Q$ besteht und dessen vier Grundpunkte $A, B, C$ und $P$ sind, und des zu ihm projektiven Strahlenbüschels $P(g)$, dessen Grundpunkt $P$ also zugleich einer der Grundpunkte des Kegelschnittbüschels ist, und wo einem Kegelschnitte $q_{i}^{2}$ des Kegelschnittbüschels, dem Polarkegelschnitt des Punktes $Q_{i}$, der durch $Q_{i}$ gehende Strahl $g_{i}$ von $P$ entspricht.
Ferner ist $P^{3}$ auch das Erzeugnis derjenigen Kegelschnittschar, welche aus den Polarkegelschnitten $G^{2}$ der sämtlichen durch den Pol $P$ von $p$ gehenden Geraden $g$ besteht und deren vier Grundtangenten die Seiten von $A B C$ und $p$ sind, und der zu ihr projektiven Punktreihe $p(Q)$, deren Träger $p$ also zugleich eine der Grundtangenten der Schar ist, und wo einem Kegelschnitt $G_{i}^{2}$ der Schar, dem Polarkegelschnitt der Geraden $g_{i}$, der zugleich auf $g_{i}$ liegende Punkt $Q_{i}$ von $p$ entspricht.

Denn der von $P$ aus durch $g_{i}$ projizierte Punkt $P_{i}$ von $p^{3}$ liegt zugleich auch auf dem Polarkegelschnitt $q_{i}^{2}$ von $Q_{i} \equiv p g^{i}$ (Nr. 56). Nun bilden aber (nach Satz 15 meiner Diss. S. 74) die Polarkegelschnitte $q^{2}$ der sämtlichen auf $p$ liegenden Punkte $Q$ einen durch $A, B, C$ und $P$ gehenden Kegelschnittbüschel, welcher zu der Punktreihe $p(Q)$ und mithin auch zu dem $p(Q)$ von $P$ aus projizierenden Strahlenbüschel $P(g)$ projektiv ist.

Endlich ist $p^{3}$ auch das Erzeugnis einer auf $(P)^{2}$, die von $A B C$ um $P$ erzeugte Involution, sich stützenden hyperbolischen Strahleninvolution $\quad(I)^{2}$, deren Doppelstrahlen also ein Paar in $(P)^{2}$ bilden, und des zu ihr projektiven Strahlenbüschels um denjenigen Punkt $P_{i}$ von $p^{3}$, welcher durch den in $(P)^{2}$ zu dem
Endlich ist $P^{3}$ auch das Erzeugnis einer auf $(p)^{2}$, die von $A B C$ auf $p$ erzeugte Involution, sich stützenden hyperbolischen Punktinvolution $(i)^{2}$, deren Doppelpunkte also ein Paar in $(p)^{2}$ bilden, und der zu ihr projektiven Punktreihe auf demjenigen Strahl $p_{i}$ von $P^{3}$, welcher von $p$ in dem in $(p)^{2}$ zu dem ersten Reprä- 
ersten Repräsentanten der Doppelstrahlen von $(I)^{2}$ zugepaarten Strahl $g_{i}$ von $P$ aus projiziert wird, und wo den die Strahlen $P A, P B$, $P C, \quad P P_{i}$ enthaltenden Paaren von $(I)^{2}$ der Reihe nach die Strahlen $P_{i} A$, $P_{i} B, P_{i} C$ und die Tangente von $p^{3}$ in $P_{i}$ im Büschel um $P_{i}$ entsprechen (wodurch die Projektivität zwischen $(I)^{2}$ und dem Büschel um $P_{i}$ immer bestimmt ist). sentanten der Doppelpunkte von $(i)^{2}$ zugepaarten Punkte $Q_{i}$ getroffen wird, und wo den die Punkte $P_{a} \equiv(p, B C)$, $P_{b} \equiv(p, C A), P_{c} \equiv(p, A B)$, $Q_{i} \equiv\left(p p_{i}\right)$ enthaltenden Paaren von $(i)^{2}$ der Reihe nach die Punkte $\left(p_{i}, B C\right),\left(p_{i}\right.$, $C A),\left(p_{i}, A B\right)$ und der Berührungspunkt von $P^{3}$ in $p_{i}$ in der Punktreihe auf $p_{i}$ entsprechen (wodurch die Projektivität zwischen $(i)^{2}$ und der Punktreihe auf $p_{i}$ immer bestimmt ist).

Dies folgt aus dem ersten Ergebnisse in Nr.63 und aus dem oben (Nr.69) Auseinandergesetzten, nämlich daß der von einer Geraden $r$ um $P_{i}$ beschriebene Strahlenbüschel $P_{i}(r)$ zu demjenigen Strahlenbüschel $P\left(g_{s}\right)$, welcher von dem vierten harmonischen Strahle $g_{s}$ zu dem festen Strahle $g_{i}$ in bezug auf das die beiden weitern Schnittpunkte von $r$ mit $p^{3}$ von $P$ aus projizierende Strahlenpaar von $(I)^{2}$ beschrieben wird, und mithin auch zu der von diesem Strahlenpaare beschriebenen Involution $(I)^{2}$ selbst projektiv ist. In dieser Projektivität entsprechen aber, weil $p^{3}$ durch $A, B, C$ und $P_{i}$ geht, den $P A, P B, P C, P P_{i}$ enthaltenden Strahlenpaaren von $(I)^{2}$ der Reihe nach die Strahlen $P_{i} A, P_{i} B, P_{i} C$ und die Tangente von $p^{3}$ in $P_{i}$ im Büschel um $P_{i}$. Hierdurch ist nun die Projektivität immer bestimmt, wenn auch etwa $P B$ und $P C$ ein Strahlenpaar in $(I)^{2}$ bilden sollen; da dann $P_{i}$ der dritte Schnittpunkt $P_{a}$ von $B C$ mit $p^{3}$ sein müssen wird und also die beiden Strahlen $P P_{i} \equiv P P_{a}$ und $P A$, die das durch $P B$ und $P C$ harmonisch getrennte Strahlenpaar von $(P)^{2}$ bilden (Nr. 4 und 5), die Doppelstrahlen von $(I)^{2}$ sein werden; mithin gehören immer die vier Strahlen $P A, P B, P C, P P_{i}$ mindestens drei voneinander verschiedenen Paaren von $(I)^{2}$ an.

Die Kurve $p^{3}$ ist auch der Ort der Berührungspunkte der aus $P$ an die
Der Büschel $P^{3}$ wird auch aus den Tangenten in den Schnittpunkten von $p$ 
sämtlichen Kegelschnitte derjenigen Schar gehenden Tangenten, welche Schar aus den Polarkegelśshnitten $G^{2}$ der durch $P$ gehenden Geraden $g$ besteht und deren Grundtangenten also die Seiten von $A B C$ und die Polare $p$ von $P$ sind. mit den sämtlichen Kegelschnitten desjenigenBüschels gebildet, welcher aus den Polarkegelschnitten $q^{2}$ der auf $p$ liegenden Punkte $Q$ besteht und dessen Grundpunkte also die Ecken von $A B C$ und der Pol $P$ von $p$ sind.

Denn nach Hilfssatz 2 (Nr.52) muß rechts die Tangente in einem Schnittpunkte $Q_{x}$ von $p$ mit etwa dem Polarkegelschnitt $q_{i}^{2}$ von $Q_{i}$ derjenige Strahl von $Q_{x}$ sein, welcher in der von $A B C$ um $Q_{x}$ erzeugten Involution $\left(Q_{x}\right)^{2}$ dem Strahle $Q_{x} Q_{i}$, also $p$, zugepaart ist, und mithin der durch $Q_{x}$ gehende Strahl von $P^{3}$. (Siehe auch meine Diss. Nr. 25, S. 70.)

82. Nunmehr wollen wir zeigen, daß auch, umgekehrt, jede beliebige Kurve dritter Ordnung mit isoliertem Doppelpunkte der Ort derjenigen Punkte ist, welche dem isolierten Doppelpunkte zugepaart sind in den von einem gewissen Dreiecke auf den sämtlichen durch den Doppelpunkt gehenden Geraden in der oben (Nr. 4) angegebenen Weise erzeugten Punktinvolutionen zweiten Grades und welche Punkte also dem isolierten Doppelpunkte konjugiert sind in bezug auf jenes Dreieck (Nr.46), und mithin eine solche Kurve ist, wie die von uns behandelte $p^{3}$, und daß dual jeder beliebige Strahlenbüschel dritter Ordnung mit isoliertem Doppelstrahle ein solcher ist, wie der von uns behandelte $P^{3}$.

Ist nämlich $C^{3}$ eine beliebige Kurve dritter Ordnung mit einem isolierten Doppelpunkte, der etwa $P$ heiße, und sind $W_{1}$, $W_{2}, W_{3}$ die bekanntlich in einer Geraden liegenden Wendepunkte von $C^{3}, w_{1}, w_{2}, w_{3}$ deren harmonische Polaren und $X, Y, Z$ der Reihe nach die Berührungspunkte der aus $W_{1}, W_{2}, W_{3}$ an $C^{3}$ gehenden Tangenten, so gehen bekanntlich $Y Z$ durch $W_{1}, Z X$ durch $W_{2}, X Y$ durch $W_{3}, u_{1}$ durch $X, w_{2}$ durch $Y, w_{3}$ durch $Z$ und es schneiden sich außerdem $u_{1}, u_{2}, w_{3}$ im Doppelpunkte $P$; mithin muß, weil $w_{1}$ von $W_{1}$ durch $W_{2}$ und $W_{3}$ und mithin durch $X Y$ und $X Z, u_{2}$ von $W_{2}$ usw. harmonisch getrennt sind, die Gerade $W_{1} W_{2} W_{3}$ die Polare von $P$ in bezug auf das Dreieck 
$X Y Z$ sein; $W_{1}, W_{2}, W_{3}$ sind also die Schnittpunkte der Seiten von $X Y Z$ mit der Polare von $P$ in bezug auf $X Y Z$. Konstruieren wir nun die dem Punkte $P$ in bezug auf das Dreieck $X Y Z$ zugehörige (Satz 33 in Nr. 78) Kurve dritter Ordnung $p^{3}$, so geht diese Kurve nach Satz 33 durch $X Y Z$, hat $P$ zum isolierten Doppelpunkt und die Schnittpunkte der Seiten von $X Y Z$ mit der Polare von $P$ in bezug auf $X Y Z$, also $W_{1}, W_{2}, W_{3}$, zu den Wendepunkten und wird in $X, Y, Z$ von $X W_{1}, Y W_{2}, Z W_{3}$ tangiert. Demnach hat $C^{3}$ neun Punkte und außerdem noch den isolierten Doppelpunkt $P$ mit $p^{3}$ gemein und muß also mit dieser identisch sein.

Wir sehen also, daß jede beliebige Kurve dritter Ordnung mit isoliertem Doppelpunkte der Ort derjenigen Punkte ist, welche dem isolierten Doppelpunkte konjugiert sind in bezug auf das Dreieck der Berührungspunkte der drei aus den Wendepunkten an die Kurve gehenden Tangenten, und also als die dem isolierten Doppelpunkte in bezug auf jenes Dreieck zugehörige Kurve dritter Ordnung aufgefaßt werden kann; und dual für einen beliebigen Strahlenbüschel dritter Ordnung mit isoliertem Doppelstrahle.

Alle für $p^{3}$ und $P^{3}$ gefundenen Resultate gelten mithin für beliebige Kurven und Strahlenbüschel dritter Ordnung mit isoliertem Doppelpunkte bzw. Doppelstrahle.

\section{§ 12.}

83. Der Hilfssatz 1 (Nr.47), auf dem sich unsere ganze Untersuchung der $p^{3}$ und $P^{3}$ stützt, gewährt uns auch ein Kriterium der Realität und der Lage der drei gemeinschaftlichen Punkte einer beliebigen durch keine Ecke von $A B C$ gehenden Geraden und $p^{3}$ bzw. der drei gemeinschaftlichen Strahlen eines beliebigen auf keiner Seite von $A B C$ liegenden Punktes und $P^{3}$.

Sind nämlich $P_{i}, P_{k}, P_{l}$ die drei Schnittpunkte irgendeiner durch keine Ecke von $A B C$ gehenden Geraden $l$ mit $p^{3}$ (und sind also nach unserer Bezeichnung $g_{i} \equiv P P_{i}, g_{k} \equiv P P_{k}$, $g_{l} \equiv P P_{l}$ und $G_{i}, G_{k}, G_{l}$ deren Pole $v_{k l} \equiv G_{k} G_{l}, v_{l i} \equiv G_{l} G_{i}$, $v_{i k} \equiv G_{i} G_{k}$ und $V_{k l}, V_{l i}, V_{i k}$ deren Pole), so müssen nach dem Hilfssatze 1 die drei Pole $V_{k l}, V_{l i}, V_{i k}$ auf $l$ liegen. Weil aber die von $A B C$ um $V_{k l}$ erzeugte Strahleninvolution $\left(V_{k l}\right)^{2} \mathrm{zu}$ den von $A B C$ auf $g_{k}$ und $g_{l}$ erzeugten Punktinvolutionen $\left(g_{k}\right)^{2}$ und 
$\left(g_{l}\right)^{2}$ perspektiv ist und ebenso $\left(V_{l i}\right)^{2} \mathrm{zu}\left(g_{l}\right)^{2}$ und $\left(g_{i}\right)^{2},\left(V_{i k}\right)^{2} \mathrm{zu}$ $\left(g_{i}\right)^{2}$ und $\left(g_{k}\right)^{2}$ (nach Satz 3 in Nr. 10) und die Gerade $l$, auf der $V_{k l}, V_{l i}, V_{i k}$ liegen, durch die zu $P$ in $\left(g_{i}\right)^{2},\left(g_{k}\right)^{2},\left(g_{l}\right)^{2}$ zugepaarten Punkte $P_{i}, P_{k}, P_{l}$ geht, so muß die Gerade $l$ zu der Verbindungsgeraden $V_{k l} P$ zugepaart sein in $\left(V_{k l}\right)^{2}$, zu $V_{l i} P$ in $\left(V_{l i}\right)^{2}$ und zu $V_{i k} P$ in $\left(V_{i k}\right)^{2}$. Es gehen daher die drei Strahlen $V_{k l} P, V_{l i} P$, $V_{i k} P$ des zu $l$ in bezug a uf $A B C$ zugehörigen Strahlenbüschels dritter Ordnung $L^{3}$ durch $P$, den isolierten Doppelpunkt von $p^{3}$.

Nun sind nach Nr.56 $P_{i} V_{k l}, P_{k} V_{l i}, P_{l} V_{i k}$ die drei Paar Schnittpunkte von $l$ mit den Polarkegelschnitten $q_{i}^{2}$ von $Q_{i} \equiv p g_{i}$, $q_{k}^{2}$ von $Q_{k} \equiv p g_{k}, q_{l}^{2}$ von $Q_{l} \equiv p g_{l}$, welche Polarkegelschnitte einem und demselben Büschel angehören, nämlich dem aus den Polarkegelschnitten der sämtlichen auf $p$ liegenden Punkte bestehenden Kegelschnittbüschel (dessen Grundpunkte $A, B, C$ und $P$ sind). Mithin sind $P_{i} V_{k l}, P_{k} V_{l i}, P_{l} V_{i k}$ drei Punktepaare einer Involution, nämlich der durch jenes Kegelschnittbüschel in $l$ eingeschnitten oder, was dasselbe ist, der durch das Viereck $A B C P$ der Grundpunkte jenes Kegelschnittbüschels auf $l$ festgelegten Involution. Es muß daher, wenn zwei der drei Punkte $P_{i}, P_{k}, P_{l}$ oder alle drei einander unendlich benachbart sind oder wenn zwei dieser drei Punkte konjugiert-imaginär sind, das Nämliche auch von den drei andern Punkten $V_{k l}, V_{l i}, V_{i k}$ und mithin auch von den diese drei Punkte mit $P$ verbindenden Strahlen $V_{k l} P, V_{l i} P, V_{i k} P$ gelten; und umgekehrt (dasselbe kann auch in derselben Weise bewiesen werden, wie oben in Nr.50). Also haben wir:

Satz 36. Eine beliebige, durch keine Ecke von $A B C$ gehende reelle Gerade $l$ hat mit $p^{3}$, der zu $P$ in bezug auf $A B C$ zugehörigen Kurve dritter Ordnung, einen reellen und zwei konjugiert-imaginäre Punkte gemein, oder drei reelle und voneinander verschiedene, oder drei reelle, von denen zwei aber unendlich benachbart sind, wo $l$ also eine Tangente an $p^{3}$ ist, oder endlich drei reelle, einander unendlich benachbarte, wo $l$ also eine der drei Wendetangenten an $p^{3}$ ist, je nachdem von den drei gemeinschaftlichen Strahlen von $P$, dem isolierten Doppelpunkte von $p^{3}$, und $L^{3}$, dem zu $l$ in bezug auf $A B C$ zugehörigen Strahlenbüschel dritter Ordnung, einer reell und die beiden andern konjugiert-imaginär sind, oder alle drei reell und 
voneinander verschieden, oder alle drei reell, deren zwei aber unendlich benachbart sind, wo $P$ also ein Berührungspunkt von $L^{3}$ ist, oder endlich alle drei reell und einander unendlich benachbart sind, wo $P$ also einer der drei Rückkehrpunkte von $L^{3}$ ist; und umgekehrt.

84. Diesem Kriterium können wir noch eine andere Fassung geben.

Nach dem Ergebnisse in Nr.62 wird nämlich jeder reelle Strahl $l_{i}$ von $L^{3}$ durch die beiden auf ihm liegenden Berührungspunkte (außer seinem eigenen) von $L^{3}$ in zwei Strecken geteilt, von denen eine, und zwar die von $l$ getroffene Strecke nur solche Punkte enthält, durch die nur ein einziger reeller Strahl von $L^{3}$, nämlich der Strahl $l_{i}$ selbst, geht, und die andere Strecke nur solche Punkte enthält, durch die drei reelle Strahlen von $L^{3}$, also außer $l_{i}$ noch zwei reelle, gehen. Weil nun die Strahlen des Büschels dritter Ordnung $L^{3}$ die ganze Ebene überdecken und stetig aufeinander folgen und die Berührungspunkte von $L^{3}$ gleichfalls stetig aufeinander folgen und eine von $L^{3}$ eingehüllte Kurve dritter Klasse bilden, so müssen auf jeder beliebigen reellen $\mathrm{Ge}-$ raden $d$ der Ebene von $L^{3}$ sowohl die nur je einen reellen Strahl von $L^{3}$ enthaltenden Punkte, die die Schnittpunkte von $d$ mit den von $l$ getroffenen Strecken der Strahlen von $L^{3}$ sind, wie auch die je drei reelle Strahlen von $L^{3}$ enthaltenden Punkte, welche letztere die Schnittpunkte von $d$ mit den von $l$ nicht getroffenen Strecken der Strahlen von $L^{3}$ sind, je stetig aufeinander folgen; und zwar werden die ersteren Punkte von den letzteren, wenn eventuell auf $d$ Punkte beider Art vorhanden sind, durch Berührungspunkte von $L^{3}$ getrennt sein müssen. Man kann daher von einem Punkte der einen Art zu keinem Punkte der andern Art gelangen, ohne die von $L^{3}$ eingehüllte Kurve dritter Klasse zu überschreiten. Mithin werden in der Ebene von $L^{3}$ die kontinuierlichen Bereiche der Punkte der einen Art von den kontinuierlichen Bereichen der Punkte der zweiten Art durch die von $L^{3}$ eingehüllte Kurve dritter Klasse, die die gemeinsame Grenze dieser Bereiche ist, getrennt. Wir können nunmehr sagen, daß die Punkte der ersten Art, nämlich die nur je einen reellen Strahl von $L^{3}$ enthaltenden Punkte, innerhalb der von $L^{3}$ eingehüllten Kurve liegen, dagegen die Punkte der zweiten Art außerhalb. 


\section{Hiernach geht der Satz 36 über in}

Satz 37. Eine beliebige, durch keine Ecke von $A B C$ gehende reelle Gerade $l$ hat mit $p^{3}$, der zu $P$ in bezug auf $A B C$ zugehörigen Kurve dritter Ordnung, einen reellen und zwei konjugiert-imaginäre Punkte gemein, oder drei reelle und voneinander verschiedene, oder ist eine einfache Tangente an $p^{3}$, oder ist endlich eine Wendetangente an $p^{3}$, je nachdem $P$, der isolierte Doppelpunkt von $p^{3}$, innerhalb, oder außerhalb, oder auf der von $L^{3}$, dem zu $l$ in bezug auf $A B C$ zugehörigen Strahlenbüschel dritter Ordnung, eingehüllten Kurve dritter Klasse liegt, ohne ein Rückkehrpunkt derselben zu sein, oder endlich ein Rückkehrpunkt dieser Kurve ist; und umgekehrt.

In jedem der letzten zwei Sätze steht die Umkehrung dem direkten dual gegenüber.

85. Die drei Punkte, in denen eine durch keine Ecke von $A B C$ gehende Gerade $l$ von $p^{3}$ geschnitten wird, und die drei durch $P$ gehenden Strahlen von $L^{3}$ stehen (nach Nr. 83) in folgendem Zusammenhang:

Satz 38. Jeder der drei Schnittpunkte einer durch keine Ecke von $A B C$ gehenden Geraden $l$ mit $p^{3}$ ist zu einem der drei Schnittpunkte von $l$ mit den durch $P$, den isolierten Doppelpunkt von $p^{3}$, gehenden Strahlen von $L^{3}$, dem zu $l$ in bezug auf $A B C$ zugehörigen Strahlenbüschel dritter Ordnung, zugepaart in der durch das vollständige Viereck $A B C P$ in $l$ eingeschnittenen Punktinvolution. Oder, was auf dasselbe herauskommt: Jeder der drei durch $P$ gehenden Strahlen von $L^{3}$ ist zu einem der drei Verbindungsgeraden von $P$ mit den auf $l$ liegenden Punkte von $p^{3}$ zugepaart in der um $P$ durch das vollständige Vierseit $a b c l$ festgelegten Strahleninvolution $(a b c \equiv A B C)$.

Dieser Satz liefert nun die erstfolgende lineare Lösung der

Aufgabe 24. Einen der drei Schnittpunkte von $p^{3}$ mit einer beliebigen, durch keine Ecke von $A B C$ gehenden Geraden $l$ zu bestimmen, wenn einer der drei durch $P$ gehenden Strahlen von $L^{3}$ bekannt ist.

Auflösung 1. Man ermittelt denjenigen Punkt auf $l$, welcher zu dem Schnittpunkte des bekannten, durch $P$ 
gehenden Strahles von $L^{3}$ mit $l$ zugepaart ist in der durch das Viereck $A B C P$ in $l$ eingeschnittenen Involution; dieser Punkt wird einer der Schnittpunkte von $l$ mit $p^{3}$ sein.

Auflösung 2. Man ermittelt die Polare (in bezug auf $A B C)$ des Schnittpunktes von $l$ mit dem bekannten, durch $P$ gehenden Strahle von $L^{3}$, bringt diese Polare zum Schnitt mit $p$, der Polare von $P$, und verbindet den letzten Schnittpunkt mit $P$ durch eine Gerade; alsdann wird der Schnittpunkt dieser Geraden mit $l$ zugleich einer der Schnittpunkte von $l$ mit $p^{3}$ sein.

Die zweite Lösung ergibt sich daraus, daß der Schnittpunkt von $l$ mit dem bekannten, durch $P$ gehenden Strahle von $L^{3}$, welcher Schnittpunkt etwa $V_{k l}$ sei, nach Nr.83 auf dem Polarkegelschnitt $q_{i}^{2}$ von $Q_{i}$ liegt und seine Polare $v_{k l}$ also durch $Q_{i}$ geht, wo $Q_{i}$ der Schnittpunkt von $p$ mit demjenigen Strahle $g_{i}$ ist, welcher den auf $l$ liegenden Punkt $P_{i}$ von $p^{3}$ mit $P$ verbindet.

Wenn also alle drei durch $P$ gehenden Strahlen von $L^{3}$ bekannt sind, kann man, wie eben gezeigt, durch lineare Konstruktionen alle drei auf $l$ liegenden Punkte von $p^{3}$ ausfindig machen. Sind aber nur zwei jener drei Strahlen bekannt, so ermittelt man auf diesem Wege zwei der drei Schnittpunkte von $l$ mit $p^{3}$, der dritte wird dann wie in der linearen Aufgabe 4 (Nr.61) gefunden. Ist endlich nur einer jener drei Strahlen bekannt, so ermittelt man einen der drei Schnittpunkte von $l$ mit $p^{3}$ wie in der linearen Aufgabe 24, sodann die beiden andern Punkte wie in der quadratischen Aufgabe 1 (Nr. 51, 61).

86. Wenden wir den Satz 37 auf die unendlich ferne Gerade $s_{\infty}$ an, was aber nur dann gestattet ist, wenn keine der Ecken von $A B C$ im Unendlichen liegt, so ergibt sich:

Satz 39. Sind alle drei Ecken von $A B C$ eigentliche Punkte der Ebene und ist $P$ irgendein auf keiner Seite von $A B C$ liegender Punkt der Ebene, so ist die zu $P$ in bezug auf $A B C$ zugehörige Kurve dritter Ordnung $p^{3}$ unvollständig hyperbolisch, überschüssig hyperbolisch oder parabolisch-hyperbolisch oder endlich eine divergierende Parabel, je nachdem $P$ innerhalb, außerhalb oder auf der- 
jenigen Kurve dritter Klasse, welche von dem zu der unendlich fernen Geraden $s_{\infty}$ in bezug auf $A B C$ zugehörigen Strahlenbüschel $S_{\infty}^{3}$ eingehüllt wird, liegt, ohne jedoch ein Rückkehrpunkt dieser Kurve zu sein, oder endlich ein Rückkehrpunkt derselben ist. Die Kurve $p^{3}$ ist sicher überschüssig hyperbolisch, wenn $P$ innerhalb des Dreiecks $A B C$ liegt.

Das letzte ergibt sich aus dem spätern Satze 40 (Nr. 87), da in diesem Falle $P$ mit dem Schwerpunkte $S$ von $A B C$, dem Pole von $s_{\infty}$ in bezug auf $A B C$, in einem und demselben Dreiecksgebiete liegen.

Weil die Wendepunkte von $p^{3}$ die Schnittpunkte von $p$, der Polare von $P$ in bezug auf $A B C$, mit den Seiten von $A B C$ sind (Satz 33 in Nr. 78) und der Pol der unendlich fernen Geraden $s_{\infty}$ in bezug auf $A B C$ der Schwerpunkt $S$ von $A B C$ ist, so erhellt:

Auf der unendlich fernen Geraden $s_{\infty}$ liegt dann und nur dann einer der drei Wendepunkte von $p^{3}$ oder alle drei, wenn $P$ auf einer der drei Mittellinien von $A B C$ liegt bzw. wenn $P$ der Schwerpunkt $S$ von $A B C$ ist.

Ist nun einer der drei durch $P$ gehenden Strahlen von $S_{\infty}^{3}$ bekannt, so können, wie in voriger Nummer gezeigt wurde, sämtliche Asymptotenrichtungen von $p^{3}$ ermittelt werden, sodann die Asymptoten selbst und die Tangentialpunkte der unendlich fernen Punkte von $p^{3}$ und also auch die Begleiterin der unendlich fernen Geraden $s_{\infty}$ wie in den Aufgaben 3 und 5 (Nr.51,61).

87. Wir wollen noch ein positives Kriterium der Realität aller drei Schnittpunkte einer reellen Geraden mit $p^{3}$ angeben; dieses lautet:

Satz 40. Jede reelle Gerade, deren Pol (in bezug auf $A B C$ ) innerhalb des $P$, den isolierten Doppelpunkt von $p^{3}$, enthaltenden Dreiecksgebietes von $A B C$ liegt, was aber dann und nur dann eintritt, wenn die Gerade in jenes Dreiecksgebiet (die Begrenzung mitgerechnet) nicht eindringt (s. Nr.1), hat mit $p^{3}$ drei reelle, und zwar voneinander verschiedene Punkte gemein.

Beweis. Ist etwa $l$ eine solche Gerade, liegt also ihr Pol $L$ mit $P$ im Innern eines und desselben Dreiecksgebietes (wonach $l$ 
sicher durch keine Ecke von $A B C$ gehen kann), so ist (nach Nr.2) der ganze Polarkegelschnitt $L^{2}$ von $l$ im Innern dieses Dreiecksgebietes enthalten, während dieses Innere ganz dem Innern des Polarkegelschnitts $p^{2}$ von $P$ angehört. Es liegen daher alle Punkte $G$ von $p^{2}$ außerhalb $L^{2}$ und folglich (s. Satz 7 meiner Dissertation, S.52) muß $l$ sämtliche Polarkegelschnitte $g^{2}$ der auf $p^{2}$ liegenden Punkte $G$ in je zwei reellen und voneinander verschiedenen Punkten schneiden. Nun muß aber die reelle Gerade $l$ mit $p^{3}$ mindestens einen reellen Punkt, etwa den von $P$ aus durch $g_{i}$ projizierten Punkt $P_{i}$, gemein haben und mithin (weil $l$, wie wir soeben sahen, den Polarkegelschnitt $g_{i}^{2}$ von $G_{i}$, von dem auf $p^{2}$ liegenden Pole von $g_{i}$, in zwei reellen und voneinander verschiedenen Punkten schneidet) nach Satz 23 in Nr. 51 noch zwei reelle und voneinander verschiedene Punkte, von denen mindestens einer, etwa der von $P$ aus durch $g_{k}$ projizierte Punkt $P_{k}$, von $P_{i}$ verschieden sein muß (der andere wäre nur dann dem $P_{i}$ unendlich benachbart, wenn einer der beiden Schnittpunkte von $l$ mit $g_{i}^{2} T_{i}$ wäre). Es muß aber auch der andere der letzten zwei gemeinschaftlichen Punkte von $l$ und $p^{3}$, der von $P_{k}$ verschiedene, von $P_{i}$ verschieden sein; denn $l$ schneidet auch den Polarkegelschnitt $g_{k}^{2}$ von $G_{k}$ in zwei reellen und voneinander verschiedenen Punkten, und folglich müssen auch die beiden Schnittpunkte (außer $P_{k}$ ) von $l$ mit $p^{3}$, welche, wie wir sahen, von $P_{k}$ verschieden sind und von denen einer $P_{i}$ ist, voneinander verschieden sein. Es sind also alle drei Schnittpunkte von $l$ mit $p^{3}$ reell und voneinander verschieden, was $\mathrm{zu}$ beweisen war.

\section{$\S 13$.}

88. Mit Hilfe der Sätze 33 (Nr. 78), 28 und 29 (Nr. 70) läßt sich nun ein neues Kriterium der Realität und der Lage der drei gemeinsamen Punkte irgendeiner Geraden und $p^{3}$ bzw. der drei gemeinsamen Strahlen irgendeines Punktes und $P^{3}$ ableiten.

Sind nämlich $P$ und $p$ Pol und Polare in bezug auf $A B C$, so besteht $P^{3}$, der zu $p$ in bezug auf $A B C$ zugehörige Strahlenbüschel dritter Ordnung, aus den Polaren der Punkte von $p^{3}$, der zu $P$ in bezug auf $A B C$ zugehörigen Kurve dritter Ordnung (Satz 33); mithin hat irgendein Punkt $K$ mit $P^{3}$ diejenigen drei Strahlen $p_{i}, p_{k}, p_{l}$ und nur diejenigen drei gemein, welche die 
Polaren (in bezug auf $A B C$ ) der drei weitern (außer den Ecken von $A B C$, die gemeinsame Punkte der $p^{3}$ und des Polarkegelschnitts eines jeden Punktes sind) gemeinsamen Punkte $P_{i}, P_{k}, P_{l}$ von $k^{2}$, dem Polarkegelschnitt von $K$ in bezug auf $A B C$, und $p^{3}$ sind.

Leiten wir nun in der in Satz 28 angegebenen Weise aus dem Polarkegelschnitt $k^{2}$, der mit $p^{3}$ die Ecken von $A B C$ gemein hat, etwa den durch die Punkte $P_{b}^{\prime} \equiv p p_{B} \equiv p(P B)$ und $P_{c}^{\prime} \equiv p p_{C} \equiv p(P C)$ und mithin nach Satz 28, weil $A$ selbst der Pol von $P A$ ist, auch durch $A$ gehenden Kegelschnitt $x^{2}$ ab, so wird nach Satz 28 $k^{2}$ mit $p^{3}$ außer den Ecken von $A B C$ noch diejenigen drei Punkte $P_{i}, P_{k}, P_{l}$ und nur diejenigen drei gemein haben, welche von $P$, dem isolierten Doppelpunkte von $p^{3}$, aus durch die Polaren $g_{i}, g_{k}, g_{l}$ (in bezug auf $A B C$ ) der drei weiteren (außer der Ecke $A$ ) gemeinsamen Punkte $G_{i}, G_{k}, G_{l}$ von $x^{2}$ und $p^{2}$, dem Polarkegelschnitt von $P$ in bezug auf $A B C$, projiziert werden.

Folglich haben wir:

I. Ein beliebiger Punkt $K$ hat mit $P^{3}$ diejenigen drei Strahlen $p_{i}, p_{k}, p_{l}$ und nur diejenigen drei gemein, welche die Polaren (in bezug auf $A B C$ ) derjenigen drei Punkte $P_{i}, P_{k}, P_{l}$ von $p^{3}$ sind, welche Punkte von $P$, dem isolierten Doppelpunkte von $p^{3}$, aus durch die Polaren $g_{i}, g_{k}, g_{l}$ (in bezug auf $A B C$ ) der drei weiteren (außer $A$ ) Schnittpunkte $G_{i}, G_{k}, G_{l}$ von $x^{2}$ und $p^{2}$ projiziert werden.

Oder, wenn man beachtet, daß nach Satz 33 die $P_{i}, P_{k}, P_{l}$ von $P$ aus projizierenden Strahlen $g_{i}, g_{k}, g_{l}$ durch die Schnittpunkte $Q_{i} \equiv p p_{i}, Q_{k} \equiv p p_{k}, Q_{l} \equiv p p_{l}$ gehen müssen, wenn $P_{i}, P_{k}, P_{l}$ die Pole von $p_{i}, p_{k}, p_{l}$ sind:

II. Ein beliebiger Punkt $K$ hat mit $P^{3}$ diejenigen drei Strahlen $p_{i}, p_{k}, p_{l}$ und nur diejenigen drei gemein, welche von $p$ in denjenigen Punkten $Q_{i}, Q_{k}, Q_{l}$ geschnitten werden, in welchen Punkten der Reihe nach $p$ zugleich von den durch $P$ gehenden Polaren $g_{i}, g_{k}, g_{l}$ (in bezug auf $A B C$ ) der drei weiteren (außer $A$ ) Schnittpunkte $G_{i}, G_{k}, G_{l}$ von $x^{2}$ und $p^{2}$ geschnitten wird,

89. Nun ist der Büschel der Polaren $l$ um den Punkt $K$ zu der krummen Punktreihe der Pole $L$ auf dem Polarkegelschnitt $k^{2}$ projektiv (Nr. 2), wo in dieser Projektivität den drei gemeinsamen 
Strahlen $p_{i}, p_{k}, p_{l}$ von $K$ und $P^{3}$ die drei weiteren (außer den Ecken von $A B C$ ) Schnittpunkte $P_{i}, P_{k}, P_{l}$ von $k^{2}$ und $p^{3}$ entsprechen müssen; da die Pole der Strahlen von $P^{3}$ auf $p^{3}$ liegen. Ferner sind (nach Nr.70) die beiden Punktreihen auf $k^{2}$ und $x^{2}$ projektiv, wenn jedem Punkte $L$ von $k^{2}$ derjenige Punkt $\Lambda$ auf $x^{2}$ zugewiesen wird, welcher von $P_{b}^{\prime}$ aus durch die Polare des zweiten Schnittpunktes von $B L$ mit $p_{b}^{\prime 2}$, dem durch $B$ gehenden Polarkegelschnitt von $P_{b}^{\prime}$, projiziert wird; wo in dieser Projektivität den drei weiteren (außer den Ecken von $A B C$ ) Schnittpunkten $P_{i}, P_{k}, P_{l}$ von $k^{2}$ und $p^{3}$ die drei weiteren (außer $A$ ) Schnittpunkte $G_{i}, G_{k}, G_{l}$ von $x^{2}$ und $p^{2}$ entsprechen müssen, da nach Nr. 70 die drei letzteren Schnittpunkte $G_{i}, G_{k}, G_{l}$ von $P_{b}^{\prime}$ aus durch diejenigen drei Geraden projiziert werden, welche die Polaren der zweiten Schnittpunkte von $p_{b}^{\prime 2}$ und der drei Verbindungsgeraden $B P_{i}, B P_{k}, B P_{l}$ sind.

Folglich ist auch der Strahlenbüschel $K(l)$ zu der krummen Punktreihe $x^{2}(\Lambda)$ projektiv, wenn jedem Strahle $l$ von $K$ derjenige Punkt $\Lambda$ von $x^{2}$ zugewiesen wird, der von $P_{b}^{\prime}$ aus durch die Polare des zweiten Schnittpunktes von $B L$ (wo $L$ der Pol von $l$ ist) und $p_{b}^{\prime 2}$ projiziert wird; und in dieser Projektivität werden den drei gemeinsamen Strahlen $p_{i}, p_{k}, p_{l}$ von $K$ und $P^{3}$ die drei weiteren (außer $A$ ) Schnittpunkte $G_{i}, G_{k}, G_{l}$ von $x^{2}$ und $p^{2}$ entsprechen.

Mithin haben wir nach I (Nr. 88), da aufeinanderfolgenden Elementen in einem von zwei projektiven Gebilden stets aufeinanderfolgende Elemente im zweiten Gebilde entsprechen müssen:

Satz 41. Sind $P$ und $p$ Pol und Polare in bezug auf $A B C, p^{3}$ und $P^{3}$ die zu $P$ bzw. der zu $p$ in bezug auf $A B C$ zugehörige Kurve bzw. Strahlenbüschel dritter Ordnung, und ist

$l$ irgendeine beliebige Gerade, so hat $l$ mit $p^{3}$

$K$ irgendein beliebigerPunkt, so hat $K$ mit $P^{3}$

einen reellen und zwei konjugiert imaginäre

Punkte

Strahlen

gemein, oder drei reelle und voneinander verschiedene, oder drei reelle, von denen zwei unendlich benachbart sind (wo also 
$l$ eine Tangente von $p^{3}$ ist),

$K$ ein Berührungspunkt von $P^{3}$ ist),

oder endlich drei reelle unendlich benachbarte (wo also $l$ eine Wendetangente von $p^{3}$ ist),

$K$ ein Rückkehrpunkt von $P^{3}$ ist),

je nachdem der aus dem Polarkegelschnitt (in bezug auf $A B C$ )

$L^{2}$ von $l$ abgeleitete, von $p_{B}^{\prime}, p_{C}^{\prime}$ und mithin auch von der Seite $a$ tangierte Kegelschnitt $\Lambda^{2}$ mit $P^{2}$, dem Polarkegelschnitt von $p$ in bezug auf $A B C$, außer $a$ noch eine reelle und zwei konjugiert-imaginäre Tangenten $k^{2}$ von $K$ abgeleitete, durch $P_{b}^{\prime}, \quad P_{c}^{\prime}$ und mithin auch durch die Ecke $A$ gehende Kegelschnitt $\varkappa^{2}$ mit $p^{2}$, dem Polarkegelschnitt von $P$ in bezug auf $A B C$, außer $A$ noch einen reellen und zwei konjugiert-imaginärePunkte gemein hat, oder drei reelle und voneinander verschiedene, oder drei reelle, von denen zwei unendlich benachbart sind (wo also $\Lambda^{2}$ von $P^{2}$ in einem nicht auf $a$ gelegenen Punkte einfach oder im Berührungspunkte von $a$

(wo also $x^{2}$ von $p^{2}$ in einem von $A$ verschiedenen Punkte einfach oder in $A$

dreipunktig berührt wird), oder endlich drei reelle unendlich benachbarte (wo also

$\Lambda^{2}$ von $P^{2}$ in einem nicht auf $a$ gelegenen Punkte dreipunktig oder im Berührungspunkte von $a$ vierpunktig berührt wird).

$x^{2}$ von $p^{2}$ in einem von $A$ verschiedenen Punkte dreipunktig oder in $A$ vierpunktig berührt wird).

Bemerkung. Eine Ausnahme hiervon machen nur (links) die durch $P$ gehenden Geraden und (rechts) die auf $p$ liegenden Punkte, indem dann die zwei gemeinsamen konjugiert-imaginären Punkte der Geraden und $p^{3}$ in einem einzigen reellen Punkte, nämlich im isolierten Doppelpunkte $P$ von $p^{3}$, zusammenfallen, und dual rechts (vgl. oben Nr. 59).

90. Den aus dem Polarkegelschnitt $k^{2}$ von $K$ abgeleiteten Kegelschnitt $x^{2}$ können wir auch, ohne Vermittlung von $k^{2}$, direkt konstruieren, wenn nur der Punkt $K$ gegeben ist. 
Nach Nr. 70 wird der abgeleitete, durch $P_{b}^{\prime}, P_{c}^{\prime}$ und mithin auch durch $A$ gehende Kegelschnitt $x^{2}$ aus dem $A B C$ umschriebenen Polarkegelschnitt $k^{2}$ dadurch gewonnen, daß man zu jedem Punkte $L$ von $k^{2}$ den Schnittpunkt $\Lambda$ der beiden durch $P_{b}^{\prime}$ bzw. durch $P_{c}^{\prime}$ gehenden Polaren der zweiten Schnittpunkte von $B L$ mit $p_{b}^{\prime 2}$. und von $C L$ mit $p_{c}^{\prime 2}$ ermittelt; der Ort dieser Schnittpunkte $A$ wird dann der Kegelschnitt $x^{2}$ sein. Nun ist die Polare eines jeden auf $B L$ liegenden Punktes von $B L$ durch die beiden übrigen Ecken $C$ und $A$ harmonisch getrennt; mithin muß die durch $K$ gehende Polare $l$ eines Punktes $L$ von $k^{2}$ und ebenso die durch $P_{b}^{\prime}$ gehende Polare des zweiten Schnittpunktes von $B L$ mit $p_{b}^{\prime 2}$ von $B L$ durch $C$ und $A$ harmonisch getrennt sein. Folglich muß die durch $P_{b}^{\prime}$ gehende Polare des zweiten Schnittpunktes von $B L$ und $p_{b}^{\prime 2}$ durch $L_{b}$, den Schnittpunkt der Dreieckseite $b \equiv C A$ und $l$, gehen und also mit der Geraden $P_{b}^{\prime} L_{b}$ identisch sein. In derselben Weise erkennt man, daß die durch $P_{c}^{\prime}$ gehende Polare des zweiten Schnittpunktes von $C L$ und $p_{c}^{\prime 2}$ mit der Geraden $P^{\prime} L_{c}$, wo $L_{c} \equiv c l \equiv(A B) l$ ist, identisch sein muß. Mithin ist $\Lambda$ der Schnittpunkt der beiden Geraden $P_{b}^{\prime} L_{b}$ und $P_{c}^{\prime} L_{c}$.

Folglich erhalten wir den abgeleiteten Kegelschnitt $x^{2}$ auch dadurch, daß wir zu jedem Strahle $l$ von $K$, der von den Dreieckseiten $b$ und $c$ in den Punkten $L_{b}$ und $L_{c}$ geschnitten wird, den Schnittpunkt $\Lambda$ der beiden Geraden $P_{b}^{\prime} L_{b}$ und $P_{c}^{\prime} L_{c}$ ermitteln; der Ort dieser Schnittpunkte $\Lambda$ wird dann $x^{2}$ sein.

Es müssen daher die beiden Schnittpunkte $\left(b, K P_{c}^{\prime}\right)$ und $\left(c, K P_{b}^{\prime}\right)$ auf $x^{2}$ liegen. Denn, wenn ein Strahl $l$ von $K$ durch $P_{c}^{\prime}$ geht, wenn also $l \equiv K P_{c}^{\prime}$ ist, muß $P_{c}^{\prime} L_{c} \equiv\left(P_{c}^{\prime}, l c\right) \equiv K P_{c}^{\prime} \equiv l$ und mithin $\Lambda \equiv\left(P_{b}^{\prime} L_{b}, P_{c}^{\prime} L_{c}\right) \equiv\left(P_{b}^{\prime} L_{b}, l\right) \equiv L_{b} \equiv l b \equiv\left(K P_{c}^{\prime}, b\right)$ sein; und ebenso muß, wenn $l \equiv K P_{b}^{\prime}$ ist, der entsprechende Punkt $\Lambda \equiv\left(K P_{b}^{\prime}, c\right)$ sein. Ferner müssen die Tangenten von $x^{2}$ in den beiden Punkten $P_{b}^{\prime}$ und $P_{c}^{\prime}$ diejenigen beiden Geraden sein, von denen die eine $P_{b}^{\prime}$ mit dem Schnittpunkte $\left(b, K P_{c}\right)$ verbindet und die andere $P_{c}^{\prime}$ mit dem Schnittpunkte $\left(c, K P_{b}\right)$, wo $P_{b} \equiv p b$ und $P_{c} \equiv p c$ ist. In der Tat liegt auf jeder durch $P_{b}^{\prime}$ gehenden Geraden $x$ außer $P_{b}^{\prime}$ noch ein zweiter Punkt von $\varkappa^{2}$, nämlich derjenige Punkt $\Lambda$, welcher dem Strahle $l \equiv(K, x b)$ entspricht, dagegen liegt auf der Geraden $P_{b}^{\prime}\left(b, K P_{c}\right)$ außer $P_{b}^{\prime}$ mehr kein Punkt von $x^{2} ;$ da, wenn $x \equiv P_{b}^{\prime}\left(b, K P_{c}\right)$ ist und mithin 
dann $l \equiv(K, x b) \equiv K\left(b, K P_{c}\right) \equiv K P_{c}$ und $L_{c} \equiv l c \equiv P_{c}$, der entsprechende Punkt $\Lambda \equiv\left(P_{b}^{\prime} L_{b}, P_{c}^{\prime} L_{c}\right) \equiv\left(P_{b}^{\prime} L_{b}, P_{c}^{\prime} P_{c}\right) \equiv\left(P_{b}^{\prime} L_{b}, p\right)$ $\equiv P_{b}^{\prime}$ sein muß; und ebenso liegt auf der Geraden $P_{c}^{\prime}\left(c, K P_{b}\right)$ außer $P_{c}^{\prime}$ mehr kein Punkt von $x^{2}$.

Wir sehen also,

daß der Kegelschnitt $x^{2}$ durch die fünf Punkte $A, P_{b}^{\prime}, P_{c}^{\prime}$, $\left(b, K P_{c}^{\prime}\right),\left(c, K P_{b}^{\prime}\right)$ geht und in $P_{b}^{\prime}$ und $P_{c}^{\prime}$ von den Geraden $P_{b}^{\prime}\left(b, K P_{c}\right)$ und $P_{c}^{\prime}\left(c, K P_{b}\right)$ tangiert wird, wodurch $x^{2}$ schon überbestimmt ist.

Zugleich sehen wir (da nach Nr. 70 die Schnittpunkte $G_{i}$, $G_{k}, G_{l}$ von $x^{2}$ und $p^{2}$ zugleich die Schnittpunkte der durch $P_{b}^{\prime}$ und $P_{c}^{\prime}$ gehenden Polaren der zweiten Schnittpunkte von $B P_{i}$ mit $p_{b}^{\prime 2}$ und von $C P_{i}$ mit $p_{c}^{\prime 2}$ bzw. von $B P_{k}$ mit $p_{b}^{\prime 2}$ und von $C P_{k}$ mit $p_{c}^{\prime 2}$ bzw. von $B P_{l}$ mit $p_{b}^{\prime 2}$ und von $C P_{l}$ mit $p_{c}^{\prime 2}$ sein müssen), daß die Aussage I (Nr. 88) auch durch die folgende ersetzt werden kann.

III. Ein beliebiger Punkt $K$ hat mit $P^{3}$ diejenigen drei Strahlen $p_{i}, p_{k}, p_{l}$ und nur diejenigen drei gemein, welche die drei Schnittpunkte der Dreieckseite $b$ mit den drei Geraden $P_{b}^{\prime} G_{i}, P_{b}^{\prime} G_{k}, P_{b}^{\prime} G_{l}$ der Reihe nach mit den drei Schnittpunkten der Dreieckseite $c$ mit den drei Geraden $P_{c}^{\prime} G_{i}$, $P_{c}^{\prime} G_{k}, P_{c}^{\prime} G_{l}$ verbinden, also $b\left(P_{b}^{\prime} G_{i}\right)$ mit $c\left(P_{c}^{\prime} G_{i}\right), b\left(P_{b}^{\prime} G_{k}\right)$ mit $c\left(P_{c}^{\prime} G_{k}\right)$ und $b\left(P_{b}^{\prime} G_{l}\right)$ mit $c\left(P^{\prime} G_{l}\right)$, wo $G_{i}, G_{k}, G_{l}$ die drei weiteren (außer $A$ ) Schnittpunkte von $x^{2}$ mit $p^{2}$ sind.

\section{Zugleich haben wir neue Lösungen einiger früheren} Aufgaben gewonnen; diese Lösungen wollen wir aber wieder nur für $p^{3}$ angeben, für $P^{3}$ müssen sie dualisiert werden.

Auflösung 2 der Aufgabe 23 (Nr. 79). Man verbindet den Schnittpunkt $l p_{C}^{\prime}$ mit der Ecke $B$ und $l p_{B}^{\prime}$ mit $C$ durch die Geraden $\left(B, l p_{C}^{\prime}\right)$ und $\left(C, l p_{B}^{\prime}\right)$, wo $p_{B}^{\prime} \equiv P P_{b} \equiv P(b p)$ und $p_{C}^{\prime} \equiv P P_{c} \equiv P(c p)$, sodann ermittelt man die drei weiteren gemeinsamen Tangenten $q_{i}, q_{k}, q_{l}$ desjenigen Kegelschnitts $\Lambda^{2}$, welcher von den fünf Geraden $a, p_{B}^{\prime}, p_{C}^{\prime},\left(B, l p_{C}^{\prime}\right)$ und $\left(C, l p_{B}^{\prime}\right)$ tangiert wird, und des Polarkegelschnitts $P^{2}$ von $p$ (in bezug auf $A B C$ ), von welchen beiden Kegelschnitten eine gemeinsame Tangente, nämlich die Dreieckseite $a$, bekannt ist, bringt dann $q_{i}, q_{k}, q_{l}$ zum Schnitt mit 
etwa $p_{B}^{\prime}$ und verbindet diese drei Schnittpunkte mit $B$ durch Gerade; alsdann werden die drei Schnittpunkte $P_{i}, P_{k}$ und $P_{l}$ dieser Geraden $B\left(p_{B}^{\prime} q_{i}\right), B\left(p_{B}^{\prime} q_{k}\right)$ und $B\left(p_{B}^{\prime} q_{l}\right)$ mit $l$ die gesuchten gemeinsamen Punkte von $l$ und $p^{3}$ sein.

Diese Lösung ergibt sich unmittelbar aus der Aussage III (Nr. 90), wenn dieselbe dualisiert wird.

Sind ein oder zwei Schnittpunkte von $l$ und $p^{3}$ bekannt, so können dementsprechend eine oder zwei der gemeinsamen Tangenten von $\Lambda^{2}$ und $P^{2}$ nach III linear ermittelt werden, und es kommt dann nur noch auf die Ermittlung der zwei noch unbekannten Tangenten bzw. der einen noch unbekannten Tangente an. Diese Ermittlung kann aber durch die folgende Bemerkung vereinfacht werden.

Das von $P$ an $\Lambda^{2}$ gehende Tangentenpaar $p_{B}^{\prime} p_{C}^{\prime}$, das von $P$ an $P^{2}$ gehende, aus den beiden konjugiert-imaginären Doppelstrahlen der von $A B C$ erzeugten Involution $(P)^{2}$ bestehende (Nr. 2) Tangentenpaar, das $P$ mit den beiden Schnittpunkten $a q_{i}$ und $q_{k} q_{l}$ verbindende Geradenpaar und das $P$ mit den beiden Schnittpunkten $a q_{k}$ und $q_{l} q_{i}$ verbindende Geradenpaar bilden vier Strahlenpaare derjenigen Involution $(I)^{2}$ um $P$, welche von dem aus den gemeinsamen Tangenten von $\Lambda^{2}$ und $P^{2}$ gebildeten vollständigen Vierseit $a q_{i} q_{k} q_{l}$ erzeugt wird; die beiden Doppelstrahlen von $(I)^{2}$ sind also $p_{A} \equiv P A$ und $p_{A}^{\prime} \equiv P P_{a}$ (wo $P_{a} \equiv a p$ ist), da $p_{A} p_{A}^{\prime}$ dasjenige Strahlenpaar von $(P)^{2}$ ist, das durch $p_{B}^{\prime}$ und $p_{C}^{\prime}$ harmonisch getrennt ist (Nr. 4). Ferner muß die Verbindungsgerade der beiden Pole der Geraden $P\left(q_{k} q_{l}\right)$ in bezug auf $\Lambda^{2}$ und $P^{2}$ durch den Schnittpunkt $q_{k} q_{l}$ gehen, da jeder jener beiden Pole von $P\left(q_{k} q_{l}\right)$ durch die beiden gemeinsamen Tangenten $q_{k}$ und $q_{l}$ der Kegelschnitte $\Lambda^{2}$ und $P^{2}$ harmonisch getrennt sein muß.

Hieraus ergeben sich nun die folgenden Lösungen:

Auflösung 3 der Aufgabe 1 (Nr. 51). Man verbindet $P_{i}$ mit den Ecken $B$ und $C$ durch die Geraden $B P_{i} \equiv p_{i B}$, $C P_{i} \equiv p_{i C}$, dann die beiden Schnittpunkte $p_{B}^{\prime} p_{i B}$ und $p_{C}^{\prime} p_{i C}$ durch die Gerade $q_{i}$; sodann ermittelt man denjenigen durch $P$ gehenden Strahl $x$, welcher von $P\left(a q_{i}\right)$, dem $P$ mit dem Schnittpunkte der Dreieckseite $a$ und $q_{i}$ verbindenden Strahle, durch $p_{A}$ und $p_{A}^{\prime}$ harmonisch getrennt ist, und dessen beide Pole $X^{\prime}$ und $X^{\prime \prime}$ in bezug auf $P^{2}$, den Polarkegelschnitt 
von $p$, und denjenigen Kegelschnitt, welcher von $a, p_{B}^{\prime}, p_{C}^{\prime}$, $q_{i}$ und etwa der Geraden $\left(B, r p_{c}^{\prime}\right)$ tangiert wird (wobei nach Nr. 2 der Pol $X^{\prime}$ von $x$ in bezug auf $P^{2}$ der Schnittpunkt von $p$ mit dem zu $x$ in $(P)^{2}$ zugepaarten Strahle sein wird), und dann die beiden aus $x\left(X^{\prime} X^{\prime \prime}\right)$, dem Schnittpunkte von $x$ und der $X^{\prime}$ mit $X^{\prime \prime}$ verbindenden Geraden, an $P^{2}$ gehenden Tangenten $q_{k}$ und $q_{l}$; alsdann verbindet man etwa die Ecke $B$ mit den beiden Schnittpunkten $q_{k} p_{B}^{\prime}$ und $q_{l} p_{B}^{\prime}$ durch die Geraden $B\left(q_{k} p_{B}^{\prime}\right)$ und $B\left(q_{l} p_{B}^{\prime}\right)$ und bringt diese beiden Geraden zum Schnitt mit der Geraden $r$ in den Punkten $P_{k}$ und $P_{l}$; diese Punkte $P_{k}$ und $P_{l}$ werden dann die gesuchten Schnittpunkte von $r$ und $p^{3}$ sein.

Auflösung 3 der Aufgabe 4 (Nr. 61). Man verbindet die beiden Punkte $P_{k}$ und $P_{l}$ von $r$ mit den Ecken $B$ und $C$ durch die Geraden $B P_{k} \equiv p_{k B}, C P_{k} \equiv p_{k C}, \quad B P_{l} \equiv p_{l B}$ und $C P_{l} \equiv p_{\imath C}$, dann die beiden Schnittpunkte $p_{B}^{\prime} p_{k B}$ und $p_{C}^{\prime} p_{k C}$ durch die Gerade $q_{k}$ und die beiden Schnittpunkte $p_{B}^{\prime} p_{l_{B}}$ und $p_{C}^{\prime} p_{l_{B}}$ durch die Gerade $q_{l}$; sodann ermittelt man die beiden durch $P$ gehenden Strahlen $x$ und $y$, welche der Reihe nach von $P\left(q_{k} q_{l}\right)$ und $P\left(a q_{k}\right)$ durch $p_{A}$ und $p_{A}^{\prime}$ harmonisch getrennt sind; alsdann verbindet man die beiden Schnittpunkte $a x$ und $q_{l} y$ durch die Gerade $q_{i}$ und darauf den Schnittpunkt $q_{i} p_{B}^{\prime}$ mit $B$ durch die Gerade $B\left(q_{i} p_{B}^{\prime}\right)$, der Schnittpunkt $P_{i}$ dieser Gerade mit $r$ wird dann der gesuchte dritte gemeinsame Punkt von $r$ und $p^{3}$ sein.

Bemerkt man nun, daß nach Satz 41 (Nr. 89) eine Gerade $l$ dann und nur dann eine Tangente an $p^{3}$ ist, wenn zwei der drei weiteren (außer a) gemeinsamen Tangenten von $\Lambda^{2}$ und $P^{2}$ unendlich benachbart sind und also $\Lambda^{2}$ von $P^{2}$ in einem nicht auf $a$ gelegenen Punkte berührt wird oder (was, wie aus I in Nr. $88 \mathrm{zu}$ entnehmen ist, nur dann eintreten kann, wenn $l$ durch den auf $a$ liegenden Wendepunkt $P_{a} \equiv p a$ geht) im Berührungspunkte von $a$ mindestens dreipunktig berührt wird; und ferner, daß, wenn $A^{2}$ von $P^{2}$ einfach oder dreipunktig berührt wird, dann in derjenigen auf $(P)^{2}$ sich stützenden Strahleninvolution $(I)^{2}$, deren Doppelstrahlen $p_{A}$ und $p_{A}^{\prime}$ sind, in der die beiden aus $P$ an $\Lambda^{2}$ gehenden Tangenten $p_{B}^{\prime}$ und $p_{C}^{\prime}$ ein Paar bilden und welche Involution also mit der von dem aus den gemeinsamen 
Tangenten von $\Lambda^{2}$ und $P^{2}$ gebildeten vollständigen Vierseit um $P$ erzeugten identisch sein muß, die beiden Geraden, welche $P$ mit dem gemeinsamen Berührungspunkte von $\Lambda^{2}$ und $P^{2}$ und dem Schnittpunkte der beiden übrigen gemeinsamen Tangenten von $\Lambda^{2}$ und $P^{2}$ verbinden bzw. welche $P$ mit dem gemeinsamen dreipunktigen Berührungspunkte von $\Lambda^{2}$ und $P^{2}$ und dem Schnittpunkte der gemeinsamen Tangente in diesem Berührungspunkte und der übrigen gemeinsamen Tangente von $\Lambda^{2}$ und $P^{2}$ verbinden, und die beiden Geraden, welche $P$ mit den beiden Schnittpunkten der Tangente im gemeinsamen Berührungspunkte (von $\Lambda^{2}$ und $P^{2}$ ) und der beiden übrigen gemeinsamen Tangenten (von $\Lambda^{2}$ und $P^{2}$ ) verbinden, je ein Strahlenpaar bilden (vgl. Nr. 72), so ergeben sich noch die folgenden Lösungen.

Auflösung 4 der Aufgabe 2 (Nr. 51). Man verbindet $P_{i}$ mit $B$ und $C$ durch die Geraden $p_{i B}$ und $p_{i C}$ und dann die beiden Schnittpunkte $p_{B}^{i} p_{i B}$ und $p_{C}^{\prime} p_{i C}$ durch die Gerade $q_{i}$; sodann ermittelt man den vierten harmonischen Strahl $x$ zu $P\left(a q_{i}\right)$, dem $P$ mit dem Schnittpunkte der Dreieckseite $a$ und $q_{i}$ verbindenden Strahle, in bezug auf $p_{A}$ und $p_{A}^{\prime}$ und darauf die beiden Schnittpunkte $U_{i_{1}}$ und $U_{i_{2}}$ von $x$ mit $P^{2}$, dem Polarkegelschnitt von $p$, und die beiden Tangenten $q_{i_{1}}$ und $q_{i_{2}}$ von $P^{2}$ in $U_{i_{1}}$ und $U_{i_{2}}$; alsdann verbindet man die Schnittpunkte $q_{i_{1}} p_{B}^{\prime}$ und $q_{i_{2}} p_{B}^{\prime}$ mit $B$ und die Schnittpunkte $q_{i_{1}} p_{C}^{\prime}$ und $q_{i_{2}} p_{C}^{\prime}$ mit $C$ und bringt dann die Verbindungsgeraden $B\left(q_{i_{1}} p_{B}^{\prime}\right)$ und $C\left(q_{i_{1}} p_{C}^{\prime}\right)$ zum Schnitt im Punkte $P_{i_{1}}$ und $B\left(q_{i_{2}} p_{B}^{\prime}\right)$ mit $C\left(q_{i_{2}} p_{C}^{\prime}\right)$ zum Schnitt im Punkte $P_{i_{2}}$. Die beiden Geraden $P_{i} P_{i_{1}}$ und $P_{i} P_{i_{2}}$ sind dann die gesuchten, von $P_{i}$ an $p^{3}$ gehenden Tangenten; und zwar sind $P_{i_{1}}$ und $P_{i_{2}}$ ihre Berührungspunkte mit $p^{3}$.

Auflösung 5 der Aufgabe 3 (Nr. 51). Man verbindet die beiden Schnittpunkte $p_{B}^{\prime} p_{i B}$ und $p_{C}^{\prime} p_{i C}$ (wo $p_{i B} \equiv B P_{i}$ und $p_{i C} \equiv C P_{i}$ sind) durch die Gerade $q_{i}$, welche Gerade eine Tangente von $P^{2}$ sein wird (nach III in Nr. 90, dualisiert); sodann ermittelt man den Berührungspunkt $U_{i}$ von $q_{i}$ mit $P^{2}$ und darauf die zweite (außer $a$ ) durch $B$ gehende Tangente $\mu$ desjenigen Kegelschnitts, welcher von den vier Geraden $a$, $p_{B}^{\prime}, p_{C}^{\prime}$ und $q_{i}$ berührt wird und $U_{i}$ zum Berührungspunkt in $q_{i}$ hat (wodurch der Kegelschnitt vollständig bestimmt ist); 
alsdann wird $P_{i}\left(\mu p_{B}^{\prime}\right)$, die $P_{i}$ mit dem Schnittpunkte $\mu p_{B}^{\prime}$ verbindende Gerade, die gesuchte Tangente von $p^{3}$ in $P_{i}$ sein.

Auflösung 6 derselben Aufgabe. Man ermittelt, ebenso wie in der vorgehenden Auflösung $5, q_{i}$ und ihren Berührungspunkt $U_{i}$ mit $P^{2}$, sodann die beiden vierten harmonischen Strahlen $x$ und $y$ zu $P U_{i}$ bzw. zu $P\left(a q_{i}\right)$, dem $P$ mit dem Schnittpunkte der Dreieckseite $a$ und $q_{i}$ verbindenden Strahle, in bezug auf $p_{A}$ und $p_{A}^{\prime}$; alsdann verbindet man die beiden Schnittpunkte $x a$ und $y q_{i}$ durch eine Gerade $q_{i_{1}}$ und bringt $B\left(q_{i_{1}} p_{B}^{\prime}\right)$, die $B$ mit dem Schnittpunkte $q_{i_{1}} p_{B}^{\prime}$ verbindende Gerade, zum Schnitt mit $C\left(q_{i_{1}} p_{C}^{\prime}\right)$, der $C$ mit dem Schnittpunkte $q_{i_{1}} p_{C}^{\prime}$ verbindenden Geraden, im Punkte $P_{i_{1}}$. Die Verbindungsgerade $P_{i} P_{i_{1}}$ wird dann die gesuchte Tangente von $p^{3}$ in $P_{i}$ sein; und zwar wird dann $P_{i_{1}}$ der Tangentialpunkt von $P_{i}$ sein.

Auflösung 7 derselben Aufgabe. Man ermittelt, ebenso wie in der Auflösung 6, $q_{i}$ und den vierten harmonischen Strahl $x$ zum Strahle $P\left(a q_{i}\right)$ in bezug auf $p_{A}$ und $p_{A}^{\prime}$, sodann die zweite (außer $q_{i}$ ) aus dem Schnittpunkte $x q_{i}$ an $P^{2}$ gehende Tangente $q_{i_{1}}$ und bringt, ebenso wie in der Auflösung 6 , die beiden Geraden $B\left(q_{i_{1}} p_{B}^{\prime}\right)$ und $C\left(q_{i_{1}} p_{C}^{\prime}\right)$ zum Schnitt im Punkte $P_{i_{1}}$; alsdann wird $P_{i_{1}}$ der Tangentialpunkt von $P_{i}$ und die Gerade $P_{i} P_{i_{1}}$ die gesuchte Tangente von $p^{3}$ in $P_{i}$ sein.

Auflösung 8 derselben Aufgabe. Man ermittelt wieder $q_{i}$, sodann die Polare $q_{i_{1}}$ (in bezug auf $A B C$ ) des Schnittpunktes $Q$ von $p$ und $q_{i}$ und bringt die beiden Geraden $B\left(q_{i_{1}} p_{B}^{\prime}\right)$ und $C\left(q_{i_{1}} p_{C}^{\prime}\right)$ zum Schnitt im Punkte $P_{i_{1}}$; alsdann wird $P_{i_{1}}$ der Tangentialpunkt von $P_{i}$ usw.

Die letzte Lösung erhellt folgendermaßen. Bezeichnet man die Tangente von $p^{3}$ in $P_{i}$ mit $\mathrm{t}_{i}$ und den Tangentialpunkt von $P_{i}$, also den weiteren (außer $P_{i}$ ) Schnittpunkt von $\mathrm{t}_{i}$ und $p^{3}$, mit $P_{x}$, und ist $T_{i}^{2}$ der aus dem Polarkegelschnitt $\mathfrak{I}_{i}^{2}$ (in bezug auf $A B C$ ) von $t_{i}$ abgeleitete, von $p_{B}^{\prime}$ und $p_{C}^{\prime}$ und mithin auch von $a$ tangierte Kegelschnitt, so müssen nach II (dualisiert) in Nr. 88 die $P_{i}$ und $P_{x}$ von $P$ aus projizierenden Geraden $g_{i}$ und $g_{x}$ der Reihe nach durch die auf $p$ liegenden Pole $Q_{i}$ und $Q_{x}$ (in bezug auf $A B C$ ) der gemeinsamen Tangenten $q_{i}$ und $q_{x}$ von $T_{i}^{2}$ und $P^{2}$ gehen. Nun 
ist aber nach der Auflösung 3 der Aufgabe 5 (Nr. 61) $g_{x}$, der den Tangentialpunkt $P_{x}$ (von $P_{i}$ ) aus $P$ projizierende Strahl, dem ersten Repräsentanten von $g_{i}$ zugepaart in der von $A B C$ erzeugten Strahleninvolution $(P)^{2}$, und mithin muß nach Satz $14(\mathrm{Nr} .23) Q_{x}$ der erste Repräsentant von $Q_{i}$, also der Schnittpunkt $Q_{i_{1}}$ von $p$ und $q_{i}$ sein. Folglich muß die weitere (außer $a$ und $q_{i}$ ) gemeinsame Tangente $q_{x}$ von $T_{i}^{2}$ und $P^{2}$, die die Polare von $Q_{x} \equiv Q_{i_{1}}$ ist, mit der in der Auflösung 8 angegebenen Polare $q_{i_{1}}$ von $Q_{i_{1}}$ und nunmehr nach III (Nr. 90) auch der Tangentialpunkt $P_{x}$ von $P_{i}$, also der weitere Schnittpunkt von $\mathrm{t}_{i}$ und $p^{3}$, mit dem in der Auflösung 8 ermittelten Punkte $P_{i_{1}}$ identisch sein.

Ist der Punkt $P_{i}$ mit einer der beiden Ecken $B$ und $C$ identisch, wo dann $p_{i B}$ bzw. $p_{i C}$ unbestimmt wird, so hat man in allen diesen Auflösungen $p_{i B}$ und $p_{B}^{\prime}$ bzw. $p_{i C}$ und $p_{C}^{\prime}$ durch $p_{i A}$ und $p_{A}^{\prime}$ zu ersetzen; und ist $P_{i}$ mit dem Wendepunkte $P a \equiv a p$ von $p^{3}$ identisch, wo dann $q_{i}$ von $a$ nicht verschieden sein wird, so hat man wieder $p_{i B}$ und $p_{B}^{\prime}$ oder $p_{i C}$ und $p_{C}^{\prime}$ durch $p_{i A}$ und $p_{A}^{\prime}$ zu ersetzen, oder man wird dann (die letzten vier Auflösungen ausgenommen) den Berührungspunkt von $a$ mit $P^{2}$ bestimmen, welcher Berührungspunkt dann als der Schnittpunkt von $a$ und $q_{\imath}$ anzusehen ist. Nur die letzten Auflösungen 6, 7, 8 versagen, wenn $P_{i}$ einer der drei Wendepunkte von $p^{3}$ ist.

\section{$\$ 14$.}

92. Wir gehen nun über zu der Untersuchung des Büschels der Tangenten von $p^{3}$ und der von $P^{3}$ eingehüllten Kurve dritter Klasse.

Sind nämlich $P$ und $p$ Pol und Polare in bezug auf $A B C$, $P^{3}$ der zu $p$ in bezug auf $A B C$ zugehörige Strahlenbüschel dritter Ordnung, und ist $l$ irgendeine Gerade, so ist ein Punkt $K$ von $l$ dann und nur dann ein einfacher Berührungspunkt bzw. ein Rückkehrpunkt von $P^{3}$, wenn $x^{2}$, der aus dem Polarkegelschnitt $k^{2}$ (in bezug auf $A B C$ ) von $K$ abgeleitete, durch $P_{b}^{\prime}, P_{c}^{\prime}$ und folglich auch durch $A$ gehende Kegelschnitt, von $p^{2}$, dem Polarkegelschnitt von $P$ in bezug auf $A B C$, in einem von $A$ verschiedenen Punkte einfach bzw. dreipunktig oder in $A$ dreipunktig bzw. vierpunktig berührt wird (Satz 41 in Nr. 89).

Nun bilden (nach Nr. 90) die aus den Polarkegelschnitten $k^{2}$ der sämtlichen auf $l$ liegenden Punkte $K$ abgeleiteten, durch $P_{b}^{\prime}$, 
$P_{c}^{\prime}$ und $A$ gehenden Kegelschnitte $x^{2}$ einen Kegelschnittbüschel $\mathfrak{L}\left(\varkappa^{2}\right)$, dessen vier Grundpunkte $A, P_{b}^{\prime}, P_{c}^{\prime}, \Lambda$ sind, wo $\Lambda$ der Schnittpunkt der beiden $P_{b}^{\prime}$ mit $L_{b} \equiv b l$ und $P_{c}^{\prime}$ mit $L_{c} \equiv c l$ verbindenden Geraden ist.

[Beiläufig bemerken wir, daß dieser Kegelschnittbüschel $\mathbb{L}\left(x^{2}\right)$ zu der geraden Punktreihe $l(K)$ projektiv ist, wenn jedem Punkte $K$ von $l$ der aus seinem Polarkegelschnitt $k^{2}$ abgeleitete $x^{2}$ zugewiesen wird. In der Tat ist (nach Nr. 90) die Tangente eines Kegelschnitts $x^{2}$ von $\mathfrak{S}\left(x^{2}\right)$ im Grundpunkte $P_{b}^{\prime}$ die $P_{b}^{\prime}$ mit dem Schnittpunkte von $b$ und $K P_{c}$ verbindende Gerade $P_{b}^{\prime}\left(b, K P_{c}\right)$, wo $K$ derjenige Punkt von $l$ ist, aus dessen Polarkegelschnitt jener Kegelschnitt $x^{2}$ abgeleitet ist. Wenn also $K$ die Gerade $l$ durchläuft, so beschreibt die Gerade $K P_{c}$ einen zu der Punktreihe $l(K)$ perspektiven Strahlenbüschel um $P_{c}$, der dann zu dem von der Tangente des dem $K$ zugehörigen Kegelschnitts $x^{2}$ um $P_{i}^{\prime}$ beschriebenen Strahlenbüschel perspektiv ist, da homologe Strahlen dieser beiden Büschel auf $b$ sich schneiden. Folglich ist die Punktreihe $l(K)$ zu dem Strahlenbüschel erster Ordnung der Tangenten der Kegelschnitte des Büschels $\mathfrak{L}\left(x^{2}\right)$ im Grundpunkte $P_{b}^{\prime}$ und mithin auch zum Kegelschnittbüschel $\mathfrak{L}\left(x^{2}\right)$ selbst projektiv.]

Wenn also $l$ kein Strahl von $P^{3}$ ist und mithin $A$ (nach III in Nr.90) kein Punkt von $p^{2}$, so geht $p^{2}$ nur durch den einen Grundpunkt $A$ des Kegelschnittbüschels $\&\left(\varkappa^{2}\right)$, da die beiden Grundpunkte $P_{b}^{\prime}$ und $P_{c}^{\prime}$ von $\mathbb{L}\left(x^{2}\right)$ auf der ganz außerhalb $p^{2}$ verlaufenden Geraden $p$ (Nr. 2) liegen, und $p^{2}$ hat daher mit jedem Kegelschnitte des Büschels $\mathfrak{L}\left(x^{2}\right)$ außer $A$ noch drei Punkte gemein, welche ein Dreieck bilden; und die Seiten dieser sämtlichen Dreiecke umhüllen bekanntlich ${ }^{1}$ ) einen und denselben und, wie man leicht einsieht, eigentlichen (nicht ausartenden) Kegelschnitt $\mathfrak{Q}^{2}$, welcher zugleich dem Dreieck $P_{b}^{\prime} P_{c}^{\prime} \Lambda$ eingeschrieben ist.

Soll nun ein Kegelschnitt $\varkappa^{2}$ des Büschels $\mathfrak{L}\left(x^{2}\right)$ in einem von $A$ verschiedenen Punkte $G_{i}$ einfach oder in einem von $A$ nicht verschiedenen Punkte $G_{i}$ dreipunktig von $p^{2}$ berührt werden, und hat folglich dann $x^{2}$ außer $A$ und $G_{i}$ noch einen von $G_{i}$ verschiedenen Punkt $G_{i}^{(1)}$ mit $p^{2}$ gemein, so wird die Tangente $t_{i}$ im Berührungspunkte $G_{i}$ von $x^{2}$ und $p^{2}$ eine Seite des aus den drei Nr. 177.

1) Siehe Steiner-Schröter, Synthetische Geometrie, Teil II, $\S 40$, 
weiteren (außer $A$ ) gemeinsamen Punkten von $x^{2}$ und $p^{2}$ gebildeten Dreiecks sein; und die beiden anderen Seiten dieses Dreiecks, welche die beiden unendlich benachbarten, im Berührungspunkte $G_{i}$ liegenden gemeinsamen Punkte von $\varkappa^{2}$ und $p^{2}$ mit $G_{i}^{(1)}$ verbinden, werden zwei unendlich benachbarte Strahlen des Punktes $G_{i}^{(1)}$ sein. Folglich wird dann $t_{i}$, die Tangente im Berührungspunkte $G_{i}$ von $x^{2}$ und $p^{2}$, auch eine Tangente von $\mathfrak{Q}^{2}$ sein, $G_{i}$ aber kein Punkt von $\Omega_{2}^{2}$; dagegen wird $G_{i}^{(1)}$, der von $G_{i}$ verschiedene gemeinsame Punkt von $x^{2}$ und $p^{2}$, auch ein Punkt von $\Omega^{2}$ sein, und zwar der Berührungspunkt der zweiten (außer $t_{i}$ ) von $G_{i}$ an $\&^{2}$ gehenden Tangente. Hat, umgekehrt, $\mathfrak{Q}^{2}$ mit $p^{2}$ die Tangente $t_{i}$ gemein, wobei aber der Berührungspunkt $G_{i}$ von $t_{i}$ mit $p^{2}$ kein Punkt von $\Omega_{2}^{2}$ ist, so muß $t_{i}$ zwei der drei weiteren (außer $A$ ) gemeinsamen Punkte desjenigen Kegelschnitts $x^{2}$ von $\mathfrak{Z}\left(x^{2}\right)$, welcher außer durch die vier Grundpunkte $A, P_{b}^{\prime}, P_{c}^{\prime}, A$ von $\mathfrak{\&}\left(x^{2}\right)$ noch durch $G_{i}$ geht (und, wenn $G_{i}$ von $A$ nicht verschieden ist, von $p^{2}$ in $A$ berürt wird), und $p^{2}$ verbinden, also müssen zwei dieser drei gemeinsamen Punkte in $G_{i}$ unendlich benachbart liegen, und der dritte dieser gemeinsamen Punkte muß der Berührungspunkt $G_{i}^{(1)}$ der zweiten (außer $t_{i}$ ) aus $G_{i}$ an $\AA^{2}$ gehenden Tangente sein; da im Berührungspunkte $G_{i}^{(1)}$ diejenigen beiden unendlich benachbarten Tangenten von $\Omega^{2}$ sich schneiden, welche die beiden unendlich benachbarten in $G_{i}$ liegenden gemeinsamen Punkte von $x^{2}$ und $p^{2}$ mit dem dritten (außer $A$ ) gemeinsamen Punkte von $x^{2}$ und $p^{2}$ verbinden. Folglich wird dann $\mathfrak{Q}^{2}$, der mit $p^{2}$ die Tangente $t_{i}$ gemein hat, mit $p^{2}$ auch denjenigen Punkt $G_{i}^{(1)}$ gemein haben, welcher der Berührungspunkt der zweiten (außer $t_{i}$ ) aus $G_{i}$ an $\S^{2}$ gehenden Tangente ist; und derjenige Kegelschnitt $x^{2}$ des Büschels $\&\left(x^{2}\right)$, welcher durch $G_{i}$ geht, wird in $G_{i}$, wenn $G_{i}$ von $A$ verschieden ist, einfach oder, wenn $G_{i}$ von $A$ nicht verschieden ist, dreipunktig von $p^{2}$ berührt werden und außerdem noch durch den gemeinsamen Punkt $G_{i}^{(1)}$ von $\Omega^{2}$ und $p^{2}$ gehen. Und hat $\&^{2}$ mit $p^{2}$ einen Punkt $G_{i}^{(1)}$ gemein, ohne daß $\mathcal{L}^{2}$ von $p^{2}$ in diesem Punkte berührt wird, so muß der zweite Schnittpunkt $G_{i}$ von $p^{2}$ mit der Tangente von $\Omega^{2}$ in $G_{i}^{(1)}$ ein einfacher Berührungspunkt, wenn $G_{i}$ von $A$ verschieden ist, oder ein dreipunktiger Berührungspunkt, wenn $G_{i}$ von $A$ nicht verschieden ist, von $p^{2}$ mit demjenigen Kegelschnitt $x^{2}$ von $\mathfrak{L}\left(x^{2}\right)$ sein, welcher außer den vier Grundpunkten $A, P_{b}^{\prime}, P_{c}^{\prime}, \Lambda$ noch den Punkt $G_{i}^{(1)}$ enthält (da die beiden unendlich benach- 
barten in der Tangente an $\Omega^{2}$ in $G_{i}^{(1)}$ liegenden Tangenten von $\Omega^{2}$ den gemeinsamen Punkt $G_{i}^{(1)}$ von $\varkappa^{2}$ und $p^{2}$ mit den zwei weiteren (außer A) gemeinsamen Punkten von $x^{2}$ und $p^{2}$ verbinden und mithin die letzteren zwei gemeinsamen Punkte in $G_{i}$ unendlich benachbart liegen müsssen), und die Tangente $t_{i}$ im Berührungspunkte $G_{i}$ von $\varkappa^{2}$ und $p^{2}$ muß dann auch eine Tangente von $\&_{2}$ sein, wobei aber $G_{i}$ kein Punkt von $\&^{2}$ sein kann, da durch $G_{i}$ außer $t_{i}$ noch die Tangente von $\mathfrak{Q}^{2}$ in $G_{i}^{(1)}$ geht.

Soll ferner ein Kegelschnitt $x^{2}$ des Büschels $\mathfrak{L}\left(x^{2}\right)$ in einem von $A$ verschiedenen Punkte $G_{i}$ dreipunktig oder in einem von $A$ nicht verschiedenen Punkte $G_{i}$ vierpunktig von $p^{2}$ berührt werden, so werden die drei Tangenten von $\mathfrak{L}^{2}$, welche je zwei der drei weiteren (außer $A$ ) gemeinsamen Punkte von $x^{2}$ und $p^{2}$ verbinden, der gemeinsamen Tangente $t_{i}$ von $x^{2}$ und $p^{2}$ in $G_{i}$ unendlich benachbart sein, und zwei dieser drei Tangenten von $\&^{2}$ schneiden sich in $G_{i}$, also muß dann $\mathfrak{Q}^{2}$ von $p^{2}$ in $G_{i}$ berührt werden (und zwar einfach). Wird, umgekehrt, $\Re^{2}$ von $p^{2}$ in $G_{i}$ berührt, so müssen die beiden unendlich benachbarten gemeinsamen Tangenten von $\Omega^{2}$ und $p^{2}$, die sich in $G_{i}$ schneiden, $G_{i}$ mit je einem der zwei weiteren (außer $A$ ) gemeinsamen Punkte desjenigen Kegelschnitts $x^{2}$ von $\mathfrak{Q}\left(x^{2}\right)$, welcher außer durch $A, P_{b}^{\prime}, P_{c}^{\prime}, \Lambda$ noch durch $G_{i}$ geht, und $p^{2}$ verbinden; folglich müssen dann die drei weiteren (außer $A$ ) gemeinsamen Punkte von $x^{2}$ und $p^{2}$ in $G_{i}$ unendlich benachbart liegen, und somit $x^{2}$ von $p^{2}$ in $G_{i}$ dreipunktig, wenn $G_{i}$ von $A$ verschieden ist, oder vierpunktig, wenn $G_{i}$ von $A$ nicht verschieden ist, berührt werden.

Mithin ist ein Punkt $K$ von $l$, wo $l$ kein Strahl von $P^{3}$ ist, dann und nur dann ein einfacher Berührungspunkt von $P^{3}$, wenn $x^{2}$ (der aus dem Polarkegelschnitt $k^{2}$ von $K$ abgeleitete, durch $A, P_{b}^{\prime}, P_{c}^{\prime}, \Lambda$ gehende Kegelschnitt) durch denjenigen $p^{2}$ nicht aber (dem eigentlichen - nicht ausartenden - Kegelschnitt) $\mathfrak{\Omega}^{2}$ angehörenden Punkt $G_{i}$ geht, welcher der Berührungspunkt von $p^{2}$ mit einer gemeinsamen Tangente $t_{i}$ von $p^{2}$ und $\Omega_{2}$ ist, oder (was nur gleichzeitig mit diesem stattfinden wird) wenn $x^{2}$ durch einen gemeinsamen Punkt $G_{i}^{(1)}$ von $p^{2}$ und $\mathfrak{S}^{2}$ geht (wobei $G_{i}^{(1)}$ der Berührungspunkt der zweiten (außer $t_{i}$ ) von $G_{i}$ an $\&^{2}$ gehenden Tangente sein müssen wird); der Punkt $K$ ist aber dann und nur dann ein Rückkehrpunkt von $P^{3}$, wenn $x^{2}$ durch einen Berührungspunkt $G_{i}$ von $p^{2}$ mit $\varrho^{2}$ geht. Dabei muß, wenn der angegebene 
Punkt $\left(G_{i}\right.$ eventuell $\left.G_{i}^{(1)}\right)$ von $A$ nicht verschieden ist, $x^{2}$ derjenige Kegelschnitt sein, welcher durch $A, P_{b}^{\prime}, P_{c}^{\prime}, \Lambda$ geht und in $A$ von $p^{2}$ berührt wird.

93. Zugleich sahen wir, daß, wenn $\&^{2}$ mit $p^{2}$ eine Tangente $t_{i}$ gemein hat, dann der Berührungspunkt $G_{i}^{(1)}$ der zweiten (außer $t_{i}$ ) aus $G_{i}$, dem Berührungspunkte von $t_{i}$ mit $p^{2}$, an $\mathfrak{Q}^{2}$ gehenden Tangente auch ein Punkt von $p^{2}$ sein muß, also $G_{i}^{(1)}$ ein gemeinsamer Punkt von $\mathfrak{Q}^{2}$ und $p^{2}$; und umgekehrt, wenn $G_{i}^{(1)}$ ein gemeinsamer Punkt von $\mathfrak{L}^{2}$ und $p^{2}$ ist, dann die Tangente $t_{i}$ von $p^{2}$ in $G_{i}$, im zweiten Schnittpunkte von $p^{2}$ mit der Tangente von $\&^{2}$ in $G_{i}^{(1)}$, auch eine Tangente von $\mathbb{R}^{2}$ sein muß; und daß dann derjenige $A P_{b}^{\prime} P_{c}^{\prime} \Lambda$ umschriebene Kegelschnitt $\varkappa^{2}$, welcher durch einen der beiden Punkte $G_{i}$ und $G_{i}^{(1)}$ geht, zugleich auch durch den zweiten dieser Punkte gehen muß und in $G_{i}$ von $p^{2}$ (einfach oder dreipunktig, je nachdem $G_{i}$ von $A$ verschieden ist oder nicht) berührt werden muß. Hierbei muß (s. oben Nr.91 die Herleitung der Auflösung 8 der Aufgabe 3) $G_{i}^{(1)}$ der Pol der Geraden $P G_{i}$ in bezug auf $A B C$ sein; da der durch $G_{i}^{(1)}$ gehende Kegelschnitt $\varkappa^{2}$ von $p^{2}$ in $G_{i}$ berührt wird (einfach bzw. dreipunktig) und mithin nach Satz 41 (Nr. 89) derjenige Punkt $K$ von $l$, durch den $p_{i}$ und $p_{i}^{(1)}$ gehen, der Berührungspunkt von $P^{3}$ in $p_{i}$ ist. Es sind daher (nach Nr.1) $G_{i}$ und $G_{i}^{(1)}$ dann und nur dann unendlich benachbarte Punkte, wenn einer und mithin auch der zweite dieser Punkte in einer der Ecken von $A B C$ zu liegen kommt. Folglich (da $\mathfrak{Q}^{2}$ von $p^{2}$ in $G_{i}$ oder in $G_{i}^{(1)}$ nur dann berührt wird, wenn $G_{i}$ und $G_{i}^{(1)}$ unendlich benachbart sind) kann $\&^{2}$ von $p^{2}$ nur in einer der Ecken von $A B C$ berührt werden, und alsdann wird $x^{2}$ von $p^{2}$ im Berührungspunkte von $\mathbb{2}^{2}$ und $p^{2}$ dreipunktig oder vierpunktig (je nachdem dieser Berührungspunkt eine der beiden Ecken $B$ und $C$ oder die Ecke $A$ ist) berührt.

94. Aus dem letzten Ergebnisse in Nr.92 und III in Nr.90 folgt nun:

I. Ist $l$ irgendeine Gerade, die aber kein Strahl von $P^{3}$ ist, $\Lambda$ der (dann nicht auf $p^{2}$ liegende) Schnittpunkt der beiden Geraden $P_{b}^{\prime} L_{b}$ und $P_{c}^{\prime} L_{c}\left(L_{b} \equiv b l, L_{c} \equiv c l\right), \mathbb{L}\left(x^{2}\right)$ der dem Viereck $A P_{b}^{\prime} P_{c}^{\prime} \Lambda$ umschriebene Kegelschnittbüschel (dessen Kegelschnitte $x^{2}$ die aus den Polarkegelschnitten $k^{2}$ 
der sämtlichen auf $l$ liegenden Punkte $K$ abgeleiteten durch $P_{b}^{\prime}$ und $P_{c}^{\prime}$ gehenden sind), $\Omega^{2}$ derjenige eigentliche (nicht ausartende) Kegelschnitt, welcher von den Seiten der sämtlichen aus den je drei weiteren (außer $A$ ) Schnittpunkten des Polarkegelschnitts $p^{2}$ (von $P$ ) und der Kegelschnitte des Büschels $\mathfrak{L}\left(x^{2}\right)$ gebildeten Dreiecke eingehüllt wird, so hat $l$ mit der von $P^{3}$ eingehüllten Kurve diejenigen vier Punkte $\mathfrak{U}_{q}, \mathfrak{U}_{r}, \mathfrak{U}_{s}, \mathfrak{U}_{t}$ und nur diejenigen vier gemein, in welchen der Reihe nach $l$ von den vier die je zwei Schnittpunkte $\left(b, P_{b}^{\prime} G_{q}\right)$ mit $\left(c, P_{c}^{\prime} G_{q}\right),\left(b, P_{b}^{\prime} G_{r}\right)$ mit $\left(c, P_{c}^{\prime} G_{r}\right)$, $\left(b, P_{b}^{\prime} G_{s}\right)$ mit $\left(c, P_{c}^{\prime} G_{s}\right)$ und $\left(b, P_{b}^{\prime} G_{t}\right)$ mit $\left(c, P_{c}^{\prime} G_{t}\right)$ verbindenden Geraden $p_{q}, p_{r}, p_{s}$ und $p_{t}$ (die vier Strahlen von $P^{3}$ sind und $\mathfrak{U}_{q}, \mathfrak{H}_{r}, \mathfrak{H}_{s}, \mathfrak{H}_{t}$ zu ihren Berührungspunkten haben) geschnitten wird, wo $G_{q}, G_{r}, G_{s}, G_{t}$ der Reihe nach die Berührungspunkte von $p^{2}$ mit den vier gemeinsamen Tangenten $t_{q}, t_{r}, t_{s}, t_{t}$ von $\mathbb{Q}^{2}$ und $p^{2}$ sind, oder (was stets dieselben vier Punkte $\mathfrak{U}_{g}, \mathfrak{U}_{r}, \mathfrak{H}_{s}, \mathfrak{H}_{t}$ liefert) in welchen Punkten $\mathfrak{U}_{q}, \mathfrak{U}_{r}, \mathfrak{H}_{s}, \mathfrak{U}_{t}$ der Reihe nach $l$ von den vier die je zwei Schnittpunkte $\left(b, P_{b}^{\prime} G_{q}^{(1)}\right)$ mit $\left(c, P_{c}^{\prime} G_{q}^{(1)}\right),\left(b, P_{b}^{\prime} G_{r}^{(1)}\right)$, mit $\left(c, P_{c}^{\prime} G_{r}^{(1)}\right),\left(b, P_{b}^{\prime} G_{s}^{(1)}\right)$ mit $\left(c, P_{c}^{\prime} G_{s}^{(1)}\right)$ und $\left(b, P_{b}^{\prime} G_{t}^{(1)}\right)$ mit $\left(c, P_{c}^{\prime} G_{t}^{(1)}\right)$ verbindenden Geraden $p_{q}^{(1)}, p_{r}^{(1)}, p_{s}^{(1)}$ und $p_{t}^{(1)}$ (die gleichfalls Strahlen von $P^{3}$ sind) geschnitten wird, wo $G_{q}^{(1)}, G_{r}^{(1)}, G_{s}^{(1)}, G_{t}^{(1)}$ die vier gemeinsamen Punkte von \&2 und $p^{2}$ sind (und wo stets nach Nr.93 $G_{q}^{(1)}, G_{r}^{(1)}, G_{s}^{(1)}, G_{t}^{(1)}$ der Reihe nach die Pole der Geraden $\stackrel{q}{P} G_{q}, P G_{r}, P G_{s}$, $P G_{t}$ in bezug auf $A B C$ sein werden). Dabei wird, wenn $\mathfrak{l}^{2}$ von $p^{2}$ in einem Punkte $G_{q}$ berührt wird (was nach Nr.93 nur dann eintreten kann, wenn $G_{q}$ eine der Ecken von $A B C$ ist), wenn also zwei der gemeinsamen Tangenten und zwei der gemeinsamen Punkte von $\mathfrak{Q}^{2}$ und $p^{2}$ in $t_{q}$ bzw. in $G_{q}$ unendlich benachbart liegen, der $G_{q}$ entsprechende gemeinsame Punkt $\mathfrak{l}_{q}$ von $l$ und der von $P^{3}$ eingehüllten Kurve ein Rückkehrpunkt von $P^{3}$ sein, also ein solcher Punkt, der für zwei Punkte der von $P^{3}$ eingehüllten Kurve $\mathrm{zu}$ zählen ist.

95. Wir wollen nun zeigen, daß der Kegelschnitt $\&^{2}$ auch, ohne Hilfe des Kegelschnittbüschels $\mathfrak{L}\left(x^{2}\right)$, direkt konstruiert werden kann, wenn nur die Gerade $l$ gegeben ist. 
Wie schon oben (Nr.92) erwähnt, wird $\&_{2}$ von den Seiten des Dreiecks $P_{b}^{\prime} P_{c}^{\prime} \Lambda$, also von den drei Geraden $P_{b}^{\prime} P_{c}^{\prime} \equiv p, P_{b}^{\prime} \Lambda$ $\equiv P_{b}^{\prime} L_{b}$ und $P_{c}^{\prime} \Lambda \equiv P_{c}^{\prime} L_{c}$ tangiert; außerdem muß aber nach I (Nr.94) $\mathfrak{\Omega}^{2}$ mit $p^{2}$ die vier Tangenten $t_{q}, t_{r}, t_{s}, t_{t}$ gemein haben, wenn $\mathfrak{U}_{q}, \mathfrak{U}_{r}, \mathfrak{U}_{s}, \mathfrak{U}_{t}$ die gemeinsamen Punkte der von $P^{3}$ eingehüllten Kurve und $l$ sind (wobei, wenn ein oder zwei dieser gemeinsamen Punkte ein bzw. zwei Rückkehrpunkte von $P^{3}$ sind, jeder Rückkehrpunkt für zwei gemeinsame Punkte zu zählen ist und auf der diesem Rückkehrpunkte entsprechenden gemeinsamen Tangente (von $\mathfrak{I}^{2}$ und $p^{2}$ ) $\mathfrak{S}^{2}$ von $p^{2}$ berührt wird). Nehmen wir nun an Stelle des dem Viereck $A P_{b}^{\prime} P_{c}^{\prime} \Lambda$ umschriebenen Kegelschnittbüschels $\mathfrak{L}\left(x^{2}\right)$, dessen Kegelschnitte $x^{2}$ die aus den sämtlichen Polarkegelschnitten $k^{2}$ der auf $l$ liegenden Punkte $K$ abgeleiteten durch $P_{b}^{\prime}, P_{c}^{\prime}$ und mithin auch durch $A$ und $A$ gehenden Kegelschnitte sind, den dem Viereck $B P_{c}^{\prime} P_{a}^{\prime} M$ umschriebenen Kegelschnittbüschel (wo $P_{a}^{\prime} \equiv p p_{A} \equiv p(P A)$ und $M$ der Schnittpunkt der beiden Geraden $P_{c}^{\prime} L_{c}$ und $P_{a}^{\prime} L_{a} \equiv P_{a}^{\prime}(a l)$ ist), dessen Kegelschnitte die aus den sämtlichen Polarkegelschnitten $k^{2}$ der auf $l$ liegenden Punkte $K$ abgeleiteten durch $P_{c}^{\prime}, P_{a}^{\prime}$ und mithin (nach Satz 28 in Nr. 70 und nach Nr.90) auch durch $B$ und $M$ gehenden Kegelschnitte sind, so wird (ganz analog wie vorher $\mathfrak{\Omega}^{2}$ ) derjenige Kegelschnitt $\mathfrak{M}^{2}$, welcher von den Seiten der sämtlichen aus den je drei weiteren (außer $B$ ) Schnittpunkten des Polarkegelschnitts $p^{2}$ (von $P$ ) und der Kegelschnitte des $B P_{c}^{\prime} P_{a}^{\prime} M$ umschriebenen Büschels gebildeten Dreiecke eingehüllt wird, von den Seiten des Dreiecks $P_{c}^{\prime} P_{a}^{\prime} M$, also von den drei Geraden $P_{c}^{\prime} P_{a}^{\prime} \equiv p, P_{c}^{\prime} M \equiv P_{c}^{\prime} L_{c}$ und $P_{a}^{\prime} M \equiv P_{a}^{\prime} L_{a}$ tangiert werden und außerdem mit $p^{2}$ die vier Tangenten $t_{q}, t_{r}, t_{s}, t_{t}$ gemein haben müssen. Folglich wird $\mathfrak{M}^{2}$ mit $\mathfrak{R}^{2}$ identisch sein müssen, da diese beiden Kegelschnitte die sechs Tangenten $p, P_{c}^{\prime} L_{c}, t_{q}, t_{r}, t_{s}, t_{t}$ gemein haben; und $\mathfrak{Q}^{2} \equiv \mathfrak{M}^{2}$ wird also von den vier Geraden $p$, $P_{a}^{\prime} L_{a}, P_{b}^{\prime} L_{b}, P_{c}^{\prime} L_{c}$ tangiert.

Nun wird $p$ von $p^{2}$ (nach Nr.2) in den beiden Doppelpunkten der von $A B C$ erzeugten Involution $(p)^{2}$ und von einem jeden Kegelschnitt des $A P_{b}^{\prime} P_{c}^{\prime} \Lambda$ umschriebenen Büschels $\mathfrak{L}\left(x^{2}\right)$ in den beiden Punkten $P_{b}^{\prime}$ und $P_{c}^{\prime}$ geschnitten; und mithin müssen die beiden Geraden, von denen die eine $A$ mit einem der drei weiteren Schnittpunkte des $p^{2}$ und irgendeines Kegelschnitts von $\mathfrak{S}\left(x^{2}\right)$ verbindet und die andere Gerade (die Tangente an $\mathfrak{L}^{2}$ ist) die 
zwei übrigen dieser Schnittpunkte, durch ein Punktepaar derjenigen auf $(p)^{2}$ sich stützenden Involution $(i)^{2}$ gehen, von welcher Involution $P_{b}^{\prime} P_{c}^{\prime}$ ein Punktepaar ist, also (weil nach Nr.4 das Punktepaar $P_{a} P_{a}^{\prime}$ von $(p)^{2}$ durch $P_{b}^{\prime}$ und $P_{c}^{\prime}$ harmonisch getrennt wird) von welcher Involution die beiden Doppelpunkte $P_{a}$ und $P_{a}$ sind. Folglich geht aus jedem Punkte $Q_{i}$ von $p$ außer $p$ selbst noch eine zweite Tangente an $\mathfrak{L}^{2}$, nämlich diejenige, welche (wenn etwa $Q_{i}^{\prime}$ der zu $Q_{i}$ in $(i)^{2}$ zugepaarte Punkt ist und der durch den zweiten Schnittpunkt der Geraden $A Q_{i}^{\prime}$ und $p^{2}$ gehende Kegelschnitt des $A P_{b}^{\prime} P_{c}^{\prime} \Lambda$ umschriebenen Büschels $\mathfrak{L}\left(x^{2}\right)$ mit $x_{i}^{2}$ bezeichnet wird) die beiden weiteren Schnittpunkte von $x_{i}^{2}$ und $p^{2}$ verbindet; dagegen geht aus dem in $(i)^{2}$ dem Schnittpunkte $(p, A \Lambda)$ zugepaarten Punkt $Q_{l}$ außer $p$ selbst mehr keine Tangente an $\mathfrak{L}^{2}$, da $\Lambda$ kein Punkt von $p^{2}$ ist und mithin derjenige durch den zweiten Schnittpunkt von $A \Lambda$ und $p^{2}$ gehende Kegelschnitt des $A P_{b}^{\prime} P_{c}^{\prime} \Lambda$ umschriebenen Büschels $\mathbb{L}\left(x^{2}\right)$, welcher Kegelschnitt mit $A \Lambda$ drei Punkte gemein hat, in das Geradenpaar $A \Lambda$ und $P_{b}^{\prime} P_{c}^{\prime} \equiv p$ ausarten muß und folglich die die beiden weiteren Schnittpunkte dieses ausgearteten Kegelschnitts und $p^{2}$ verbindende Gerade die Gerade $p$ selbst ist. Mithin ist der in $(i)^{2}$ dem Schnittpunkte $(p, A \Lambda)$ zugepaarte Punkt $Q_{l}$, also der vom Schnittpunkte $(p, A \Lambda)$ durch $P_{a}$ und $P_{a}^{\prime}$ harmonisch getrennte Punkt, der Berührungspunkt der Tangente $p$ von $\&^{2}$.

Wir sehen also, daß der Kegelschnitt $\mathcal{Q}_{2}$ von den vier Geraden $p, P_{a}^{\prime} L_{a}$, $P_{b}^{\prime} L_{b}, P_{c}^{\prime} L_{c}$ tangiert wird und denjenigen Punkt $Q_{l}$ auf $p$ zum Berührungspunkt hat, welcher vom Schnittpunkte $(A \Lambda, p)$ durch $P_{a}$ und $P_{a}^{\prime}$ harmonisch getrennt wird; wodurch $\&^{2}$ vollständig bestimmt ist.

96. Nunmehr können wir den folgenden Satz aufstellen:

Satz 42. Sind in bezug auf $A B C P$ und $p$ Pol und Polare,

$P^{2}$ der Polarkegelschnitt von $p, p^{3}$ die zu $P$ zugehörige Kurve dritter Ordnung, $K$ irgendein beliebiger Punkt, der aber auf $p^{3}$ nicht liegt, $k_{A}, k_{B}, k_{C}$ die Verbindungs- $p^{2}$ der Polarkegelschnitt von $P, P^{3}$ der zu $p$ zugehörige Strahlenbüschel dritter Ordnung, $l$ irgendeine beliebige Gerade, die aber kein Strahl von $P^{3}$ ist, $L_{a}, L_{l}, L_{c}$ die 
geraden von $K$ mit den Eckpunkten $A, B, C, g_{K}$ diejenige durch $P$ gehende Gerade, die vom Schnittpunkte $a\left(p_{B}^{\prime} k_{B}, \quad p_{C}^{\prime} k_{C}\right)$ durch die beiden (durch $P$ gehenden) Geraden $p_{A}$ und $p_{A}^{\prime}$ harmonisch getrennt ist, $\mathfrak{k}^{2} \mathrm{der}$ jenige Kegelschnitt, der durch die vier Punkte $P$, $p_{A}^{\prime} k_{A}, p_{B}^{\prime} k_{B}, p_{C}^{\prime} k_{C}$ hindurch geht und $g_{K}$ zur Tangente in $P$ hat, und bezeichnet man die Geraden, welche drei veränderliche Punkte $P_{1}, P_{2}, P_{3}$ von $p^{3}$ mit $P$ verbinden, mit $g_{1}, g_{2}, g_{3}$, die Schnittpunkte $p g_{1}, p g_{2}, p g_{3}$ mit $Q_{1}, Q_{2}, Q_{3}$ und die $P^{2}$ tangierenden Polaren (bezüglich $A B C$ ) von $Q_{1}, Q_{2}$, $Q_{3}$ mit $q_{1}, q_{2}, q_{3}$, so liegen je drei solche Punkte $P_{1}$, $P_{2}, P_{3}$ von $p^{3}$ und nur solche drei mit $K$ in je einer Geraden, für die das $P^{2}$ umschriebene Dreieck $q_{1} q_{2} q_{3}$ (der entsprechenden Tangenten von $P^{2}$ ) zugleich $\mathfrak{f}^{2}$ eingeschrieben ist.

Die Punktetripel von $p^{3}$, die mit $K$ in je einer Geraden liegen, werden von $P$ aus durch Strahlentripel einer kubischen Involution projiziert.
Schnittpunkte von $l$ mit den Seiten $a, b, c$ von $A B C$, $Q_{l}$ derjenige Punkt von $p$, der von der Geraden $A\left(P_{b}^{\prime} L_{b}\right.$, $P_{c}^{\prime} L_{c}$ ) durch die beiden (auf $p$ liegenden) Punkte $P_{a}$ und $P_{a}^{\prime}$ harmonisch getrennt ist, $\&^{2}$ derjenige Kegelschnitt, der von den vier Geraden $p, P_{a}^{\prime} L_{a}, P_{b}^{\prime} L_{b}, P_{c}^{\prime} L_{c}$ tangiert wird und $Q_{l}$ zum $\mathrm{Be}-$ rührungspunkte in $p$ hat, und bezeichnet man die Punkte, in denen drei veränderliche Strahlen $p_{1}, p_{2}, p_{3}$ von $P^{3}$ von $p$ geschnitten werden, mit $Q_{1}, Q_{2}, Q_{3}$, die Geraden $P Q_{1}, P Q_{2}, P Q_{3}$, mit $g_{1}, g_{2}, g_{3}$ und die auf $p^{2}$ liegenden Pole (bezüglich $A B C$ ) von $g_{1}, g_{2}, g_{3}$ mit $G_{1}, G_{2}, G_{3}$, so schneiden sich je drei solche Strahlen $p_{1}, p_{2}, p_{3}$ von $P^{3}$ und nur solche drei in je einem Punkte von $l$, für die das $p^{2}$ eingeschriebene Dreieck $G_{1} G_{2} G_{3}$ (der entsprechenden Punkte von $p^{2}$ ) zugleich \&2 umschrieben ist.

Die Strahlentripel von $P^{3}$, von denen jedes durch einen Punkt von $l$ geht, werden von $p$ in den Punktetripeln einer kubischen Involution geschnitten.

Denn rechts gehen dann und nur dann $p_{1}, p_{2}, p_{3}$ durch einen Punkt $K$ von $l$, wenn der aus dem Polarkegelschnitt $k^{2}$ von $K$ 
abgeleitete, durch $P_{b}^{\prime}, P_{c}^{\prime}$ und $A$ gehende Kegelschnitt $x^{2}$ mit $p^{2}$ außer $A$ noch die Punkte $G_{1}, G_{2}, G_{3}$ gemein hat (II in Nr. 88), also (nach Nr. 92) dann und nur dann, wenn die Seiten des Dreiecks $G_{1} G_{2} G_{3} \mathfrak{Q}^{2}$ tangieren. Was ferner die kubische Involution anbetrifft, vgl. weiter unten Ende Nr.102.

97. Aus dem in Nr.95 gefundenen Resultate ergibt sich nun: Ist die Gerade $l$ kein Strahl von $P^{3}$, wo dann der Schnittpunkt $\Lambda \equiv\left(P_{b}^{\prime} L_{b}, P_{c}^{\prime} L_{c}\right)$ kein Punkt von $p^{2}$ und mithin $\mathfrak{R}^{2}$, wie schon oben (Nr.92) erwähnt, ein eigentlicher (nicht ausartender) Kegelschnitt ist, so können die drei Geraden $P_{a}^{\prime} L_{a}, P_{b}^{\prime} L_{b}$ und $P_{c}^{\prime} L_{c}$, die drei Tangenten von $\&^{2}$ sind, nicht durch einen und denselben Punkt gehen. Ist aber $l$ ein Strahl von $P^{3}$, so muß dann $\Lambda \equiv\left(P_{b}^{\prime} L_{b}, P_{c}^{\prime} L_{c}\right)$ nach III (Nr.90) ein Punkt von $p^{2}$ sein, und zwar nach II (Nr. 88) derjenige Punkt von $p^{2}$, welcher der Pol (in bezug auf $A B C$ ) der $P$ mit dem Schnittpunkte von $p$ und $l$ verbindenden Geraden ist; alsdann muß aber ganz analog, wenn wir $P_{a}^{\prime}$ an Stelle von $P_{c}^{\prime}$ nehmen, auch der Schnittpunkt der beiden Geraden $P_{a}^{\prime} L_{a}$ und $P_{b}^{\prime} L_{b}$ derjenige Punkt von $p^{2}$ sein, welcher der Pol (in bezug auf $A B C$ ) der $P$ mit dem Schnittpunkte $(p l)$ verbindenden Geraden ist. Es schneiden sich also dann alle drei Geraden $P_{a}^{\prime} L_{a}, P_{b}^{\prime} L_{b}, P_{c}^{\prime} L_{c}$ in einem und demselben Punkte, nämlich im Pole (in bezug auf $A B C$ ) der Geraden $(P, p l)$.

Mithin haben wir das folgende Kriterium gewonnen:

Satz 43. Ist $p^{3}$ die zu $P$ in bezug auf $A B C$ zugehörige Kurve dritter Ordnung und $K$ irgendein Punkt, so ist $K$ dann und nur dann ein Punkt von $p^{3}$, wenn die drei Schnittpunkte $\left(p_{A}^{\prime} k_{A}\right)$, $\left(p_{B}^{\prime} k_{B}\right),\left(p_{C}^{\prime} k_{C}\right)$ auf einer und derselben Geraden liegen; und zwar ist dann diese Gerade die $P^{2}$, den Polarkegelschnitt der Polare $p$ von $P$, tangierende Polare des Schnittpunktes $(p, K P)$, welcher letzte Punkt dann
Ist $P^{3}$ der zu $p$ in bezug auf $A B C$ zugehörige Strahlenbüschel dritter Ordnung und $l$ irgendeine Gerade, so ist $l$ dann und nur dann ein Strahl von $P^{3}$, wenn die drei Geraden $P_{a}^{\prime} L_{a}, P_{b}^{\prime} L_{b}$, $P_{c}^{\prime} L_{c}$ durch einen und denselben Punkt gehen; und zwar ist dann dieser Punkt der auf $p^{2}$, dem Polarkegelschnitt des Pols $P$ von $p$, liegende Pol der Geraden $P(l p)$, welche letzte Gerade dann auch den $\operatorname{Pol} L$ von $l$ 
auch auf der Polare $k$ von $K$ liegt und der Repräsentant des Punktepaares $P, K$ ist. enthält und der Repräsentant des Strahlenpaares $p, l$ ist.

Letztes ergibt sich aus der Bemerkung, daß wenn $l$ ein Strahl von $P^{3}$ ist, auf der Geraden $(P, p l)$ auch der Pol $L$ (in bezug auf $A B C$ ) von $l$ liegen muß (Satz 33 in Nr. 78).

Beachtet man nun, daß eine Gerade $l$ dann und nur dann ein Strahl von $P^{3}$ ist, wenn $p$ und $l$ einander zugepaart sind in der von $A B C$ um ihren Schnittpunkt $(p l)$ erzeugten Strahleninvolution zweiten Grades (Nr.46), und daß $p$ eine durch keine Ecke von $A B C$ gehende Gerade ist (ebenda), so ergibt sich aus Satz 43, indem $p$ und $l$ als irgend zwei beliebige Gerade, von denen aber mindestens eine durch keine Ecke von $A B C$ geht, aufgefaßt werden:

Satz 44. Zwei Punkte $K$ und $L$, von denen mindestens einer auf keiner Seite von $A B C$ liegt,
Zwei Strahlen $m$ und $n$, von denen mindestens einer durch keine Ecke von $A B C$ geht,

sind dann und nur dann einander zugepaart in der von $A B C$

auf ihrer Verbindungsgeraden $K L$ erzeugten Punktinvolution zweiten Grades, wenn die drei Schnittpunkte $\left(k_{A}^{\prime} l_{A}\right),\left(k_{B}^{\prime} l_{B}\right),\left(k_{C}^{\prime} l_{C}\right)$ um ihren Schnittpunkt ( $m n$ ) erzeugten Strahleninvolution zweiten Grades, wenn die drei Geraden $M_{a}^{\prime} N_{a}, M_{b}^{\prime} N_{b}$, $M_{c}^{\prime} N_{c}$

und folglich (wie man sofort einsieht) auch die drei

Schnittpunkte $\left(k_{A} l_{A}^{\prime}\right),\left(k_{B} l_{B}^{\prime}\right)$, $\left(k_{C} l_{C}^{\prime}\right)$ auf einer und derselben Geraden liegen; und zwar ist dann diese Gerade die Polare (in bezug auf $A B C)$ des Schnittpunktes $(k l)$, welcher letzte dann auf der Geraden $K L$ liegt und derRepräsentant desPunktepaares $K, L$ ist; hierbei sind $k_{A} \equiv K A, k_{B} \equiv K B, k_{C} \equiv K C$, $k_{A}^{\prime}$ der vierte harmonische
Geraden $M_{a} N_{a}^{\prime}, M_{b} N_{b}^{\prime}, M_{c} N_{c}^{\prime}$ durch einen und denselben Punkt gehen; und zwar ist dann dieser Punkt der Pol (in bezug auf $A B C$ ) der Geraden $M N$, welche letzte dann durch den Schnittpunkt $(m n)$ geht und der Repräsentant des Strahlenpaares $m, n$ ist; hierbei sind $\boldsymbol{M}_{\boldsymbol{a}} \equiv m a, \boldsymbol{M}_{b} \equiv m b, \boldsymbol{M}_{c} \equiv m \boldsymbol{c}$, $\boldsymbol{M}_{a}^{\prime}$ der vierte harmonische 
Strahl zu $k_{A}$ in bezug auf $k_{B}$ und $k_{C}$ usw., $l_{A} \equiv L A$ usw. und endlich $k$ und $l$ die Polaren von $K$ und $L$ in bezug auf $A B C$.
Punkt zu $M_{a}$ in bezug auf $M_{b}$ und $M_{c}$ usw., $N_{a} \equiv n a$ usw. und endlich $M$ und $N$ die Pole von $m$ und $n$ in bezug auf $A B C$.

98. Ist aber die Gerade $l$ ein reeller gewöhnlicher Strahl von $P^{3}$, so liegen auf $l$ sein eigener Berührungspunkt, in welchem $l$ die von $P^{3}$ eingehüllte Kurve einfach berührt, und noch zwei reelle vom ersteren und voneinander verschiedene einfache Berührungspunkte von $P^{3}$; und dasselbe (abgesehen von der Realität der Berührungspunkte) gilt auch, wenn $l$ ein imaginärer Strahl von $P^{3}$ ist (s. oben Nr.62 und Ende Nr.59). Ist ferner $l$ ein Rückkehrstrahl von $P^{3}$, so liegt auf $l$ sein eigener Berührungspunkt, in welchem ein Rückkehrpunkt (also ein mit zwei Punkten der von $P^{3}$ eingehüllten Kurve äquivalenter Punkt) und ein einfacher Punkt derselben Kurve unendlich benachbart liegen, und noch ein reeller vom ersteren verschiedener einfacher Berührungspunkt von $P^{3}$ (Nr.62). Ist endlich $l$ der isolierte Doppelstrahl $p$ von $P^{3}$, so berührt $l$ die von $P^{3}$ eingehüllte Kurve doppelt, nämlich in den beiden konjugiert-imaginären Doppelpunkten der von $A B C$ erzeugten Involution $(p)^{2}$ (Satz 33 in Nr.78). Und zwar haben wir nach der Auflösung 7 der Aufgabe 3 und der Auflösung 4 der Aufgabe 2 in Nr.91 (dualisiert):

II. Ist $l$ irgendein Strahl von $P^{3}, A$ der Schnittpunkt der beiden Geraden $P_{b}^{\prime} L_{b}$ und $P_{c}^{\prime} L_{c}$, welcher Punkt dann auch auf der Geraden $P_{a}^{\prime} L_{a}$ und auf $p^{2}$ liegt (Satz 43), $Q_{l}$ der von der Geraden $A \Lambda$ durch $P_{a}$ und $P_{a}^{\prime}$ harmonisch getrennte Punkt von $p$, so wird die von $P^{3}$ eingehüllte Kurve von $l$ in demjenigen Punkte $\mathfrak{U}$ einfach berührt, in dem $l$ von der die beiden Schnittpunkte $\left(b, P_{b}^{\prime} G\right)$ und $\left(c, P_{c}^{\prime} G\right)$ verbindenden Geraden (die auch selbst ein Strahl von $P^{3}$ ist) geschnitten wird, wo $G$ der zweite (außer $\Lambda$ ) Schnittpunkt der Geraden $Q_{l} \Lambda$ und $p^{2}$ ist; außerdem hat die von $P^{3}$ eingehüllte Kurve mit $l$ diejenigen beiden Punkte $\mathfrak{H}_{1}$ und $\mathfrak{H}_{2}$ und nur diejenigen beiden gemein, in denen $l$ von den beiden die je zwei Schnittpunkte $\left(b, P_{b}^{\prime} G_{1}\right)$ mit $\left(c, P_{c}^{\prime} G_{1}\right),\left(b, P_{b}^{\prime} G_{2}\right)$ mit $\left(c, P_{c}^{\prime} G_{2}\right)$ verbindenden Geraden $p_{1}$ und $p_{2}$ (die zwei Strahlen von $P^{3}$ sind und $\mathfrak{H}_{1}, \mathfrak{H}_{2}$ zu ihren Berührungspunkten haben) 
geschnitten wird, wo $G_{1}$ und $G_{2}$ die Berührungspunkte der beiden aus $Q_{l}$ an $p^{2}$ gehenden Tangenten $t_{1}$ und $t_{2}$ sind. Hierbei wird, wenn eine der letzten beiden Tangenten $t_{1}$ und $t_{2}$ mit der Geraden $Q_{l} \Lambda$ zusammenfällt (wo dann $l$ außer dem Berührungspunkt $\mathfrak{U}$ mit der von $P^{3}$ eingehüllten Kurve nur noch einen von $\mathfrak{U}$ verschiedenen Punkt dieser Kurve enthalten und also ein Rückkehrstrahl von $P^{3}$ sein wird), einer der beiden im Berührungspunkte $\mathfrak{U}$ der von $P^{3}$ eingehüllten Kurve und $l$ unendlich benachbart liegenden Punkte ein Rückkehrpunkt von $P^{3}$ sein.

Diese Aussage ist der Aussage I (Nr. 94) ähnlich; denn, wenn $l$ ein Strahl von $P^{3}$ ist und mithin die drei Geraden $P_{a}^{\prime} L_{a}, P_{b}^{\prime} L_{b}$, $P_{c}^{\prime} L_{c}$ durch $\Lambda$ gehen, muß der von diesen drei Geraden tangierte und $p$ im Punkte $Q_{l}$ berührende Kegelschnitt $\mathfrak{L}^{2}$ in das Punktepaar $A, Q_{l}$ ausarten.

99. Wie wir sehen, hat jede Gerade, mag sie ein Strahl von $P^{3}$ sein oder nicht, vier Punkte mit der von $P^{3}$ eingehüllten Kurve gemein; diese Kurve ist also von der vierten Ordnung. Dual ist der Büschel der Tangenten der Kurve $p^{3}$ von der vierten Ordnung, und $p^{3}$ ist daher eine Kurve vierter Klasse.

Zugleich haben wir nach Satz 43 (Nr. 97), I (Nr. 94), II (Nr.98) und Nr.95 das folgende Kriterium gewonnen.

Satz 45. Ist $p^{3}$ die dem Punkte $P$ in bezug auf $A B C$ zugehörige Kurve dritter Ordnung, $K$ irgendein reeller Punkt, $x$ die Verbindungsgerade der beiden Schnittpunkte $p_{B}^{\prime} k_{B}$ und $p_{C}^{\prime} k_{C}, g_{K}$ der vom Schnittpunkte $a x$ durch $p_{A}$ und $p_{A}^{\prime}$ harmonisch getrennte Strahl von $P, P^{2}$ der Polarkegelschnitt der Polare $p$ von $P$ in bezug auf $A B C$, und liegt der Schnittpunkt $p_{A}^{\prime} k_{A}$ nicht auf $x$, so gehen dann und nur dann durch $K$ keine unend-
Ist $P^{3}$ der der Geraden $p$ in bezug auf $A B C$ zugehörige Strahlenbüschel dritter Ordnung, $l$ irgendeine reelle Gerade, $\Lambda$ der Schnittpunkt der beiden Geraden $P_{b}^{\prime} L_{b}$ und $P_{c}^{\prime} L_{c}, Q_{l}$ der von der Geraden $A \Lambda$ durch $P_{a}$ und $P_{a}^{\prime}$ harmonisch getrennte Punkt von $p, p^{2}$ der Polarkegelschnitt des Poles $P$ von $p$ in bezug auf $A B C$, und geht die Gerade $P_{a}^{\prime} L_{a}$ nicht durch $A$, so hat dann und nur dann $l$ keine unendlich benachbarte Punkte mit der 
lich benachbarte Tangenten an $p^{3}$ ( $K$ ist also kein Punkt von $p^{3}$ ); alsdann gehen von $K$ an $p^{3}$. 1) zwei Paar konjugiert-imaginärer einfacher Tangenten, oder 2) ein von $P^{3}$ eingehüllten Kurve vierter Ordnung gemein; alsdann hat $l$ mit dieser Kurve 1) zwei Paar konjugiert-imaginärer einfacher Punkte gemein, oder 2) ein

Paar konjugiert-imaginärer einfacher und zwei reelle voneinander verschiedene einfache, oder 3) vier reelle voneinander verschiedene einfache, oder 4) ein Paar konjugiertimaginärer einfacher und
eine Wendetangente,
einen Rückkehrpunkt,

oder 5) zwei reelle voneinander verschiedene einfache und eine Wendetangente, oder endlich 6) zwei Wendetangenten, je nachdem $\mathfrak{k}^{2}$, der dem Viereck

$$
P\left(p_{A}^{\prime} k_{A}\right)\left(p_{B}^{\prime} k_{B}\right)\left(p_{C}^{\prime} k_{C}\right)
$$

umschriebene und in $P$ von $g_{K}$ tangierte Kegelschnitt, mit $P^{2}$ einen Rückkehrpunkt, oder endlich 6) zwei Rückkehrpunkte, je nachdem $\mathfrak{\Omega}^{2}$, der dem Vierseit

$$
p\left(P_{a}^{\prime} L_{a}\right)\left(P_{b}^{\prime} L_{b}\right)\left(P_{c}^{\prime} L_{c}\right)
$$
eingeschriebene und $p$ in $Q_{l}$ berührende Kegelschnitt, mit $p^{2}$

1) zwei Paar konjugiert-imaginärer Tangenten oder Punkte gemein hat, oder 2) ein Paar konjugiert-imaginärer und zwei reelle voneinander verschiedene, oder 3) vier reelle voneinander verschiedene, oder 4) ein Paar konjugiert-imaginärer und zwei reelle unendlich benachbarte, oder 5) vier reelle, von denen zwei unendlich benachbart und die übrigen zwei von diesen und voneinander verschieden sind, oder endlich 6) vier reelle, von denen zweimal zwei unendlich benachbart sind, wo also

$$
\mathfrak{f}^{2} \text { mit } P^{2}
$$

$\Re^{2}$ mit $p^{2}$

eine doppelte reelle Berührung hat.

Liegt aber auch der Schnittpunkt $p_{A}^{\prime} k_{A}$ auf $x$, so gehen von $K$ an $p^{3}$ vier reelle Tangenten,
Geht aber $P_{a}^{\prime} L_{a}$ durch $\Lambda$, so hat $l$ mit der von $P^{3}$ eingehüllten Kurve vier reelle Punkte,

von denen zwei unendlich benachbart und die übrigen zwei von diesen und voneinander verschieden sind (wo also 
$K$ ein gewöhnlicher Punkt von $p^{3}$

$l$ eine gewöhnliche Tangente dieser Kurve

ist), oder zwei reelle unendlich benachbarte, von denen eine eine Wendetangente und die andere eine einfache Tangente ist, und noch eine von diesen verschiedene reelle Tangente (wo also $K$ ein Wendepunkt von $p^{3}$ sein wird), je nachdem der Schnittpunkt $\left(g_{K} x\right)$ kein Punkt von $P^{2}$ ist oder nicht.

einer ein Rückkehrpunkt und der andere ein einfacher Punkt ist, und noch einen von diesen verschiedenen reellen Punkt (wo also $l$ eine Rückkehrtangente dieser Kurve sein wird) gemein, je nachdem die Gerade $Q_{l} \Lambda$ keine Tangente von $p^{2}$ ist oder nicht.

Hierbei sei nochmals (s. oben Nr.93) hervorgehoben, daß $\mathbb{S}_{2}$ und $p^{2}$ (und ebenso $\mathfrak{k}^{2}$ und $P^{2}$ ) stets ebensoviel reelle Tangenten, wie reelle Punkte gemein haben.

100. Nunmehr können wir das folgende negative Kriterium der Imaginärität der sämtlichen Schnittpunkte einer Geraden und der von $P^{3}$ eingehüllten Kurve aufstellen.

Satz 46. Liegt ein Punkt $K$ nicht in demjenigen der vier durch $A B C$ getrennten Gebiete der Ebene, innerhalb dessen $P$ liegt, so müssen mindestens zwei der von $K$ an $p^{3}$ gehenden Tangenten reell sein.
Dringt eine Gerade $l$ in dasjenige Dreiecksgebiet von $A B C$ ein, welches den $\mathrm{Pol} P$ von $p$ in bezug auf $A B C$ enthält, so müssen mindestens zwei der Schnittpunkte von $l$ und der von $P^{3}$ eingehüllten Kurve reell sein.

In der Tat liegt das $P$ enthaltende Dreiecksgebiet ganz innerhalb des Polarkegelschnitts $p^{2}$ (Nr.2), und mithin können, wenn $l$ in dieses Dreiecksgebiet eindringt, mindestens zwei der Schnittpunkte $L_{a}, L_{b}, L_{c}$ von $l$ und den Seiten von $A B C$, durch welche die Tangenten $P_{a}^{\prime} L_{a}, P_{b}^{\prime} L_{b}, P_{c}^{\prime} L$ von $\mathfrak{L}^{2}$ gehen und welche Schnittpunkte also sicher keine inneren Punkte von $\mathfrak{L}^{2}$ sind, sicher keine äußeren Punkte von $p^{2}$ sein. Es kann also dann $p^{2}$ nicht ganz im Innern von $\mathfrak{L}^{2}$ liegen, und mithin müssen $p^{2}$ und $\mathfrak{\Omega}^{2}$ mindestens zwei reelle Punkte gemein haben; denn $\Omega^{2}$ und $p^{2}$, die nie reelle 
Tangenten, ohne reelle Punkte, gemein haben, können bekanntlich nicht auseinander liegen, und der Kegelschnitt $\&^{2}$, der von der ganz außerhalb $p^{2}$ liegenden (Nr. 2) Geraden $p$ berührt wird, kann nicht ganz im Innern von $p^{2}$ liegen. Folglich muß dann nach Satz $45 l$ mit der von $P^{3}$ eingehüllten Kurve mindestens zwei reelle Punkte gemein haben.

Für die Entscheidung über die Realität der Schnittpunkte einer Geraden und der von $P^{3}$ eingehüllten Kurve wird manchmal die folgende Bemerkung von Nutzen sein.

Der Schnittpunkt der drei Geraden $A\left(P_{b}^{\prime} L_{b}, P_{c}^{\prime} L_{c}\right)$, $B\left(P_{c}^{\prime} L_{c}, P_{a}^{\prime} L_{a}\right), C\left(P_{a}^{\prime} L_{a}, P_{b}^{\prime} L_{b}\right)$ liegt auf $p^{2}$ und innerhalb $\&^{2}$.

In der Tat gehen durch den Schnittpunkt $Q_{l} \equiv l p$ der isolierte Doppelstrahl $p$ und noch ein einziger einfacher Strahl $p_{l}$ von $P^{3}$; mithin muß nach I in Nr. 88 (s. auch Bemerkung zu Satz 41 in Nr. 89) $x_{l}^{2}$, der aus dem Polarkegelschnitt $q_{l}^{2}$ von $Q_{l}$ abgeleitete durch $P_{b}^{\prime}, P_{c}^{\prime}, A$ und $\left(P_{b}^{\prime} L_{b}, P_{c}^{\prime} L_{c}\right) \equiv \Lambda$ gehende Kegelschnitt, durch die konjugiert-imaginären Schnittpunkte von $p$ und $p^{2}$ und den auf $p^{2}$ liegenden $\mathrm{Pol} G_{l}$ von $g_{l} \equiv P Q_{l}$ (in bezug auf $A B C$ ) gehen. Nunmehr muß aber $x_{l}^{2}$, weil er mit $p$ vier Punkte, nämlich $P_{b}^{\prime}, P_{c}^{\prime}$ und die beiden konjugiert-imaginären Schnittpunkte von $p$ und $p^{2}$ gemein hat, in das Geradenpaar $p$ und $A\left(P_{b}^{\prime} L_{b}, P_{c}^{\prime} L_{c}\right)$ ausarten; folglich muß $G_{l}$ der zweite Schnittpunkt (außer $A$ ) der Geraden $A\left(P_{b}^{\prime} L_{b}, P_{c}^{\prime} L_{c}\right)$ und $p^{2}$ sein. In derselben Weise erkennt man, wenn $P_{c}^{\prime}$ und $P_{a}^{\prime}$ an Stelle von $P_{b}^{\prime}$ und $P_{c}^{\prime}$ genommen werden (wo dann $L_{b}$, $L_{c}$ und $A$ durch $L_{c}, L_{a}$ und $B$ ersetzt werden müssen), daß der Pol $G_{l}$ von $g_{l} \equiv P Q_{l}$ der zweite Schnittpunkt von $B\left(P_{c}^{\prime} L_{c}, P_{a}^{\prime} L_{a}\right)$ und $p^{2}$ sein muß; ebenso muß aber, $G_{l}$ der zweite Schnittpunkt von $C\left(P_{a}^{\prime} L_{a}, P_{b}^{\prime} L_{b}\right)$ und $p^{2}$ sein. Nunmehr müssen die Seiten des Dreiecks der drei weiteren (außer A) Schnittpunkte von $p^{2}$ mit $x_{l}^{2}$, dem ausgearteten Kegelschnitt des dem Viereck $P_{b}^{\prime} P_{c}^{\prime} A\left(P_{b}^{\prime} L_{b}, P_{c}^{\prime} L_{c}\right)$ umschriebenen Büschels $\&\left(\varkappa^{2}\right)$, drei Tangenten des Kegelschnitts $\mathfrak{Q}^{2} \operatorname{sein}(\mathrm{Nr} .92)$; mithin gehen durch $G_{l}$ zwei konjugiert-imaginäre Tangenten von $\Omega^{2}$, nämlich die beiden $G_{l}$ mit den konjugiert-imaginären Schnittpunkten von $p$ und $p^{2}$ verbindenden Geraden, und $G_{l} \mathrm{mu}$ daher innerhalb $\&^{2}$ liegen. Wir sehen also, daß die drei Geraden $A\left(P_{b}^{\prime} L_{b}, P_{c}^{\prime} L_{c}\right), B\left(P_{c}^{\prime} L_{c}, P_{a}^{\prime} L_{a}\right)$, $C\left(P_{a}^{\prime} L_{a}, P_{b}^{\prime} L_{b}\right)$ in den auf $p^{2}$ und innerhalb $\Omega_{2}^{2}$ liegenden Punkt $G_{l}$ konvergieren, was zu beweisen war. 
101. Wir können nun auch das folgende Kriterium aufstellen. Satz 47. Sind $P_{i}, P_{k}$, $P_{l}, P_{m}$ vier Punkte von $p^{3}$, $q_{i}, \quad q_{k}, \quad q_{l}, q_{m}$ diejenigen Tangenten an $P^{2}$ (dem Polarkegelschnitt der Polare $p$ von $P$ in bezug auf $A B C$ ), welche die je zwei Schnittpunkte $p_{B}^{\prime} p_{i B}$ und $p_{C}^{\prime} p_{i C}$ bzw. $p_{B}^{\prime} p_{k B}$ und $p_{C}^{\prime} p_{k C}$ bzw. $p_{B}^{\prime} p_{l B}$ und $p_{C}^{\prime} p_{l C}$ bzw. $p_{B}^{\prime} p_{m B}$ und $p_{C}^{\prime} p_{m C}\left(p_{i B} \equiv B P_{i}, p_{i C} \equiv C P_{i}\right.$, $p_{k B} \equiv B P_{k}, \quad p_{k C} \equiv C P_{k}, \quad p_{l B}$ $\equiv B P_{l}, p_{l C} \equiv C P_{l}, p_{m B} \equiv B P_{m}$, $p_{m c} \equiv C P_{m}$ ) verbinden (und welche Tangenten die $\mathrm{Po-}$ laren der vier Schnittpunkte $Q_{i}, Q_{k}, Q_{l}, Q_{m}$ von $p$ und der Verbindungsgeraden $g_{i}$ $\equiv P P_{i}, \quad g_{k} \equiv P P_{k}, \quad g_{l} \equiv P P_{l}$, $g_{m} \equiv P P_{m}$ in bezug auf $A B C$ sein müssen), $q_{i}^{(1)}, q_{k}^{(1)}, q_{l}^{(1)}$, $q_{m}^{(1)}$ die gleichfalls $P^{2}$ tangierenden Polaren der vier Punkte $Q_{i}^{(1)} \equiv p q_{i}, \quad Q_{k}^{(1)} \equiv p q_{k}$, $Q_{l}^{(1)} \equiv p q_{l}, Q_{m}^{(1)} \equiv p q_{m}$ in bezug auf $A B C, U_{i}, U_{k}, U, U_{m}$ die Berührungspunkte von $P^{2}$ mit den Tangenten $q_{i}, q_{k}$, $q_{l}, q_{m}$, so konvergieren die vier Tangenten von $p^{3}$ in $P_{i}, P_{k}, P_{l}, P_{m}$ dann und nur dann in einen Punkt, wenn $P, U_{i}, U_{k}, U_{l}, U_{m}$, $q_{i} q_{i}^{(1)}, \quad q_{k} q_{k}^{(1)}, \quad q_{l} q_{l}^{(1)}, \quad q_{m} q_{m}^{(1)}$ neun Punkte eines und desselben Kegelschnitts sind, wo dann $q_{i}^{(1)}, q_{k}^{(1)}, q_{l}^{(1)}, q_{m}^{(1)}$ die Tangenten dieses Kegel-

Sind $p_{q}, p_{r}, p_{s}, p_{t}$ vier Strahlen von $P^{3}, G_{q}, G_{r}$, $G_{s}, \quad G_{t}$ diejenigen auf $p^{2}$ (dem Polarkegelschnitt des Pols $P$ von $p$ in bezug auf $A B C$ ) liegenden Punkte, in denen die je zwei Geraden $P_{b}^{\prime} P_{q b}$ und $P_{c}^{\prime} P_{q c}$ bzw. $P_{b}^{\prime} P_{r b}$ und $P_{c}^{\prime} P_{r c}$ bzw. $P_{b}^{\prime} P_{s b}$ und $P_{c}^{\prime} P_{s c}$ bzw. $P_{b}^{\prime} P_{t b}$ und $P_{c}^{\prime} P_{t c}$ $\left(P_{q b} \equiv p_{q} b, \quad P_{q c} \equiv p_{q} c, \quad P_{r b}\right.$ $\equiv p_{r} b, \quad P_{r c} \equiv p_{r} c, \quad P_{s b} \equiv p_{s} b$, $\left.P_{s c} \equiv p_{s} c, P_{t b} \equiv p_{t} b, P_{t c} \equiv p_{t} c\right)$ sich schneiden (und welche Punkte die Pole der vier $P$ mit den Schnittpunkten $Q_{q} \equiv p p_{q}, \quad Q_{r} \equiv p p_{r}, Q_{s} \equiv p p_{s}$, $Q_{t} \equiv p p_{t} \quad$ verbindenden $\mathrm{Ge}-$ raden $g_{q}, g_{r}, g_{s}, g_{t}$ in bezug auf $A B C$ sein müssen), $G_{q}^{(1)}, G_{r}^{(1)}, \quad G_{s}^{(1)}, \quad G_{t}^{(1)}$ die gleichfalls auf $p^{2}$ liegenden Pole der vier Geraden $g_{q}^{(1)}$ $\equiv P G_{q}, g_{r}^{(1)} \equiv P G_{r}, g_{s}^{(1)} \equiv P G_{s}$, $g_{t}^{(1)} \equiv P G_{t}$ in bezug auf $A B C$, $t_{q}, t_{r}, t_{s}, t_{t}$ die Tangenten an $p^{2}$ in $G_{q}, G_{r}, G_{s}, G_{t}$, so liegen die vierBerührungspunkte von $p_{q}, p_{r}, p_{s}, p_{t}$ mit der von $P^{3}$ eingehüllten Kurve dann und nur dann in einer Geraden, wenn $p$, $t_{q}, t_{r}, t_{s}, t_{t}, G_{q} G_{q}^{(1)}, G_{r} G_{r}^{(1)}$, $G_{s} G_{s}^{(1)}, G_{t} G_{t}^{(1)}$ neun Tangenten eines und desselben Kegelschnitts sind, wo dann $G_{q}^{(1)}, G_{r}^{(1)}, G_{s}^{(1)}, G_{t}^{(1)}$ die Berührungspunkte dieses Kegel- 
schnitts in den Punkten $q_{i} q_{i}^{(1)}$, $q_{k} q_{k}^{(1)}, \quad q_{l} q_{l}^{(1)}, \quad q_{m} q_{m}^{(1)} \quad$ sein werden.

schnitts mit den Tangenten $G_{q} G_{q}^{(1)}, \quad G_{r} G_{r}^{(1)}, \quad G_{s} G_{s}^{(1)}$, $G_{t} G_{t}^{(1)}$ sein werden.

Denn liegen die Berührungspunkte von $p_{q}, p_{r}, p_{s}, p_{t}$ mit der von $P^{3}$ eingehüllten Kurve in einer Geraden $l$, so müssen nach I (Nr.94) $t_{g}, t_{r}, t_{s}, t_{t}$ und dann (nach Nr. 93) auch $G_{q} G_{q}^{(1)}, G_{r} G_{r}^{(1)}, G_{s} G_{s}^{(1)}$ $G_{t} G_{t}^{(1)}$ Tangenten eines und desselben Kegelschnitts $\&^{2}$ sein, welcher letzte auch von $p$ tangiert (Nr. 95) und von den Tangenten $G_{q} G_{q}^{(1)}$, $G_{r} G_{r}^{(1)}, G_{s} G_{s}^{(1)}, G_{t} G_{t}^{(1)}$ in bzw. $G_{q}^{(1)}, G_{r}^{(1)}, G_{s}^{(1)}, G_{t}^{(1)}$ berührt wird (Nr. 93). Und, umgekehrt, wenn $p, t_{q}, t_{r}, t_{s}, t_{t}, G_{q} G_{q}^{(1)}, G_{r} G_{r}^{(1)}$, $G_{s} G_{s}^{(1)}, G_{t} G_{t}^{(1)}$ einen und denselben Kegelschnitt tangieren, so muß dieser Kegelschnitt mit demjenigen Kegelschnitt \&2 identisch sein, welcher aus der die beiden Berührungspunkte der Strahlen $p_{q}$ und $p_{r}$ von $P^{3}$ verbindenden Geraden $l$ in der in I (Nr. 94) angegebenen Weise hervorgeht; da diese beiden Kegelschnitte nach I (Nr.94) und Nr.93 die fünf Tangenten $p, t_{q}, t_{r}, G_{q} G_{q}^{(1)}$, $G_{r} G_{r}^{(1)}$ gemein haben. Folglich muß dann nach I (Nr.94) $l$ auch durch die Berührungspunkte der Strahlen $p_{s}$ und $p_{t}$ von $P^{3}$ gehen.

102. Nach dem soeben aufgestellten Satze müssen die Kegelschnitte $\mathfrak{Q}^{2}$, welche aus allen durch den Berührungspunkt $\mathfrak{U}_{r}$ eines von den Rückkehrstrahlen verschiedenen Strahles $p_{r}$ von $P^{3}$ gehenden Geraden $l$ in der in I (Nr.94) angegebenen Weise hervorgehen, sämtlich von den drei Geraden $p, t_{r}, G_{r} G_{r}^{(1)}$ berührt werden, und zwar von $G_{r} G_{r}^{(1)}$ im Punkte $G_{r}^{(1)}$ und also eine Kegelschnittschar $\mathfrak{u}_{r}\left(\Omega^{2}\right)$ bilden, von der zwei Grundtangenten in $G_{r} G_{r}^{(1)}$ unendlich benachbart liegen. Nunmehr hat jeder Kegelschnitt der Schar $\mathfrak{u}_{r}\left(\mathfrak{Q}^{2}\right)$ mit dem Polarkegelschnitt $p^{2}$, der nur von der einen Grundtangente $t_{r}$ dieser Schar tangiert wird, noch drei weitere Tangenten gemein, welche ein Dreieck bilden; und die Eckpunkte dieser sämtlichen Dreiecke liegen (vgl. oben Nr.92) auf einem Kegelschnitt $x^{2}$, welcher durch $G_{r}^{(1)}$ geht und von $p$ im Schnittpunkte $\left(p, G_{r} G_{r}^{(1)}\right)$ berührt wird und welcher, wie wir sofort zeigen werden, außer $G_{r}^{(1)}$ noch die Eckpunkte von $A B C$ mit $p^{2}$ gemein haben muß. Denn verbindet man $\mathfrak{H}_{r}$ mit dem Rückkehrpunkte des Rückkehrstrahles $p_{A} \equiv P A$ von $P^{3}$ durch eine Gerade $l$, so muß nach I in Nr.94 (weil die $P$ mit dem Schnittpunkte $\left(p p_{A}\right)$ verbindende Gerade mit $p_{A}$ zusammenfällt und $A$ der Pol von $p_{A}$ in bezug auf $A B C$ ist) der aus dieser Geraden $l$ 
hervorgehende Kegelschnitt $\mathfrak{Q}^{2}$ von $p^{2}$ in $A$ berührt werden, und mithin ist $A$ ein Schnittpunkt von zwei weiteren (außer $t_{r}$ ) gemeinsamen unendlich benachbarten Tangenten dieses Kegelschnitts $\&^{2}$ und $p^{2}$, also ein Punkt von $x^{2}$; ebenso müssen aber auch $B$ und $C$ Punkte von $x^{2}$ sein. Der Schnittpunkt $\left(p, G_{r} G_{r}^{(1)}\right)$ ist aber nach Satz 18 (Nr.28) der Pol der Geraden $g_{r}$ (welche $P$ mit dem Schnittpunkte $Q_{r} \equiv p p_{r}$ verbindet und die Polare von $G_{r}$ in bezug auf $A B C$ ist) in bezug auf $p^{2}$ und mithin (nach Nr.2) derjenige Punkt $Q_{r}^{\prime}$ von $p$, der zu $p g_{r} \equiv Q_{r} \equiv p p_{r}$ zugepaart ist in der von $A B C$ erzeugten Punktinvolution $(p)^{2}$. Folglich muß der Polarkegelschnitt $u_{r}^{\prime 2}$ von $U_{r}^{\prime}$ in bezug auf $A B C$ (wo $U_{r}^{\prime}$ der Berührungspunkt des Polarkegelschnitts $P^{2}$ von $p$ mit der $P^{2}$ tangierenden Polare $q_{r}^{\prime}$ von $Q_{r}^{\prime}$ in bezug auf $A B C$ ist), welcher $A B C$ umschrieben ist und (weil $U_{r}^{\prime}$ auf $q_{r}^{\prime}$ liegt) durch $Q_{r}^{\prime} \equiv\left(p, G_{r} G_{r}^{(1)}\right)$ geht und (weil $U_{r}^{\prime}$ auf dem Polarkegelschnitt $P^{2}$ von $p$ liegt) von $p$ in $Q_{r}^{\prime}$ berührt wird (s. Satz 7 meiner Dissertation, S. 52), mit dem obigen Kegelschnitt $x^{2}$ identisch sein.

$\mathrm{Zu}$ bemerken sei noch, daß in $U_{r}^{\prime}$ (weil $u_{r}^{\prime 2} \equiv x^{2}$ durch den Pol $G_{r}^{(1)}$ von $g_{r}^{(1)} \equiv P G_{r}$ in bezug auf $A B C$ geht) der erste Repräsentant $g_{r}^{(1)}$ von $g_{r}$ und (weil nach Hilfssatz 2 in Nr. 52, dualisiert, der Berührungspunkt $U_{r}^{\prime}$ der Tangente $q_{r}^{\prime}$ des Polarkegelschnitts $P^{2}$ von $p$ dem Schnittpunkte $\left(p q_{r}^{\prime}\right)$ zugepaart ist in der von $A B C$ erzeugten Involution $\left(q_{r}^{\prime}\right)^{2}$, nach Nr. 5 die von $A B C^{\prime}$ erzeugte Strahleninvolution $\left(Q_{r}^{\prime}\right)^{2} \mathrm{zu}\left(q_{r}^{\prime}\right)^{2}$ perspektiv ist und mithin $Q_{r}^{\prime} U_{r}^{\prime}$ zu $p$ in $\left(Q_{r}^{\prime}\right)^{2}$ zugepaart und also ein Strahl von $P^{3}$ ist) der durch $Q_{r}^{\prime}$ gehende Strahl von $P^{3}$ konvergieren müssen.

Beachtet man nun, daß die Seiten eines jeden der $p^{2}$ umschriebenen und $x^{2} \equiv u_{r}^{\prime}{ }^{2}$ eingeschriebenen Dreiecke, welche Seiten die drei weiteren (außer $t_{r}$ ) gemeinsamen Tangenten eines Kegelschnitts $\mathfrak{Q}^{2}$ der Schar $\mathfrak{u}_{r}\left(\mathfrak{Q}^{2}\right)$ und $p^{2}$ sind, nach I (Nr. 94) $p^{2}$ in den Polen (in bezug auf $A B C$ ) solcher drei durch $P$ gehenden Geraden berühren müssen, welche drei Geraden durch die Schnittpunkte von $p$ mit solchen drei Strahlen von $P^{3}$, deren Berührungspunkte (mit der von $P^{3}$ eingehüllten Kurve) in einer durch $\mathfrak{l}_{r}$ gehenden Geraden liegen, hindurchgehen, so ergibt sich folgendes Resultat.

Satz 48. Ist $\mathfrak{U}_{r}$ der Berührungspunkt irgendeines von den Rückkehrstrahlen verschiedenen Strahles $p_{r}$ von $P^{3}$, 
$Q_{r}$ der Schnittpunkt von $p$ und $p_{r}, Q_{r}^{\prime}$ der zu $Q_{r}$ in der von $A B C$ erzeugten Punktinvolution $(p)^{2}$ zugepaarte Punkt, $q_{r}^{\prime}$ die dem Polarkegelschnitt $P^{2}$ von $p$ tangierende Polare von $Q_{r}^{\prime}$ in bezug auf $A B C, U_{r}^{\prime}$ der Berührungspunkt von $q_{r}^{\prime}$ und $P^{2}, u_{r}^{\prime}$ der Polarkegelschnitt von $U_{r}^{\prime}$ und $p^{2}$ der Polarkegelschnitt des Pols $P$ von $p$ (alles in bezug auf $A B C$ ), und bezeichnet man die Punkte, in denen drei veränderliche Strahlen $p_{1}, p_{2}, p_{3}$ von $P^{3}$ von $p$ geschnitten werden, der Reihe nach mit $Q_{1}, Q_{2}, Q_{3}$, die Verbindungsgeraden $P Q_{1}, P Q_{2}, P Q_{3}$ mit $g_{1}, g_{2}, g_{3}$, die auf $p^{2}$ liegenden Pole (in bezug auf $A B C$ ) von $g_{1}, g_{2}, g_{3}$ mit $G_{1}, G_{2}, G_{3}$, wo nach Satz 43 (Nr. 97) in $G_{1} P_{a}^{\prime} P_{1 a}, P_{b}^{\prime} P_{1 b}, P_{c}^{\prime} P_{1 c}$, in $G_{2} P_{a}^{\prime} P_{2 a}$, $P_{b}^{\prime} P_{2 b}, P_{c}^{\prime} P_{2 c}$ und in $G_{3} P_{a}^{\prime} P_{3 a}, P_{b}^{\prime} P_{3 b}, P_{c}^{\prime} P_{3 c}\left(P_{1 a} \equiv p_{1} a\right.$, $P_{1 b} \equiv p_{1} b, P_{1 c} \equiv p_{1} c, P_{2 a} \equiv p_{2} a$ usw.) konvergieren müssen, und die Tangenten von $p^{2}$ in $G_{1}, G_{2}, G_{3}$ mit $t_{1}, t_{2}, t_{3}$, so liegen die Berührungspunkte je dreier solcher Strahlen $p_{1}, p_{2}, p_{3}$ von $P^{3}$ und nur solcher drei Strahlen mit $\mathfrak{l}_{r}$ in je einer Geraden, für die das $p^{2}$ umschriebene Dreieck $t_{1} t_{2} t_{3}$ (der entsprechenden drei Tangenten von $p^{2}$ ) zugleich $u_{r}^{\prime 2}$ eingeschrieben ist; und dual für die mit einer gegebenen Tangente von $p^{3}$ in je einen Punkt konvergierenden Tangenten von $p^{3}$.

Die Strahlentripel von $P^{3}$, deren Berührungspunkte mit $\mathfrak{H}_{r}$ in je einer Geraden liegen, werden von $p$ in den Punktetripeln derjenigen kubischen Involution geschnitten, welche durch die zwei Punktetripel $Q_{r} Q_{r^{\prime}}^{\prime} Q_{r^{\prime \prime}}^{\prime}$ und $Q_{r}^{\prime} Q_{r}^{(1)^{\prime}} Q_{r}^{(1)^{\prime}}$ (wo also $Q_{r}^{(1)^{\prime}}$ ein Doppelpunkt der kubischen Involution sein wird) festgelegt ist; wobei $Q_{r^{\prime}}^{\prime}$ und $Q_{r^{\prime \prime}}^{\prime}$ dasjenige Punktepaar in $(p)^{2}$ bilden, dessen Repräsentant $Q_{r}^{\prime}$ ist, und $Q_{r}^{(1)}$ derjenige Punkt von $p$ ist, der dem ersten Repräsentanten $Q_{r}^{(1)}$ von $Q_{r}$ zugepaart ist in $(p)^{2}$; und dual für $p^{3}$.

Das letztere erhellt folgendermaßen: Die Tripel der Seiten $t_{1} t_{2} t_{3}$ der $p^{2}$ um - und $u_{r}^{\prime 2}$ eingeschriebenen Dreiecke bilden bekanntlich eine kubische Involution im Büschel der Tangenten von $p^{2}$, mithin auch die Punktetripel $G_{1} G_{2} G_{3}$ in der zu diesem Tangentenbüschel perspektiven Punktreihe auf $p^{2}$ und nunmehr auch die Strahlentripel $g_{1} g_{2} g_{3}$ in dem zu dieser Punktreihe der Pole auf $p^{2}$ projektiven Büschel der Polaren um $P$ und die 
Punktetripel $Q_{1} Q_{2} Q_{3}$ in der zu diesem Büschel um $P$ perspektiven Punktreihe auf $p$. In dieser kubischen Punktinvolution auf $p$ müssen nun die Schnittpunkte $Q_{r} Q_{r^{\prime}}^{\prime} Q_{r^{\prime \prime}}^{\prime}$ von $p$ mit denjenigen Strahlen $p_{r}, p_{r^{\prime}}^{\prime}$ und $p_{r^{\prime \prime}}^{\prime \prime}$ von $P^{3}$, deren Berührungspunkte nach $I$ in Nr. 54 (dualisiert) mit $\mathfrak{H}_{r}$ in einer Geraden, nämlich auf $p_{r}$ selbst, liegen, und die Schnittpunkte $Q_{r}^{\prime}$ und $Q_{r}^{(1)^{\prime}}$ von $p$ mit denjenigen Strahlen $p_{r}^{\prime}$ und $p_{r}^{(1)^{\prime}}$ von $P^{3}$, deren Berührungspunkte nach I (Nr. 54) mit $\mathfrak{H}_{r}$ in einer Geraden, nämlich auf $p_{r}^{(1)}$, liegen (wo, weil im Berührungspunkte von $p_{r}^{(1)^{\prime}}$ zwei unendlich benachbarte gemeinsame Punkte von $p_{r}^{(1)^{\prime}}$ und der von $P^{3}$ eingehüllten Kurve liegen, $p_{r}^{(1)^{\prime}}$ und mithin auch $Q_{r}^{(1)^{\prime}}$ doppelt zu zählen ist), je ein Punktetripel bilden.

103. Ebenso aber wie die aus den sämtlichen durch $\mathfrak{U}_{r}$, den Berührungspunkt eines von den Rückkehrstrahlen verschiedenen Strahles $p_{r}$ von $P^{3}$, gehenden Geraden $l$ in der in I (Nr.94) angegebenen Weise hervorgehenden Kegelschnitte $\mathfrak{Q}^{2}$, bilden auch die aus den sämtlichen durch irgendeinen ganz beliebigen Punkt $K$ gehenden Geraden $l$ in der nämlichen Weise hervorgehenden Kegelschnitte $\mathfrak{Q}^{2}$ eine Schar $\mathfrak{\mathfrak { l }}\left(\mathfrak{\Omega}^{2}\right)$, deren Grundtangenten nach Nr. 92 aus $p$ und den drei Seiten des Dreiecks der drei weiteren (außer $A$ ) gemeinsamen Punkte des Polarkegelschnitts $p^{2}$ von $P$ und $x^{2}$, des aus dem Polarkegelschnitt $k^{2}$ von $K$ abgeleiteten durch $P_{b}^{\prime}$ und $P_{c}^{\prime}$ gehenden Kegelschnitts, bestehen.

[Beiläufig bemerken wir, daß diese Kegelschnittschar $\mathfrak{f}\left(\mathfrak{Q}^{2}\right)$ zum Strahlenbüschel $K(l)$ projektiv ist, wenn jeder Geraden $l$ von $K$ der aus ihr hervorgehende Kegelschnitt $\mathfrak{Q}^{2}$ zugewiesen wird. Denn, wenn $l$ den Strahlenbüschel $K(l)$ durchläuft, so beschreiben die beiden Geraden $P_{b}^{\prime} L_{b}$ und $P_{c}^{\prime} L_{c}$, wo $L_{b} \equiv l b$ und $L_{c} \equiv l c$ ist, zwei zu $K(l)$ perspektive Strahlenbüschel um $P_{b}^{\prime}$ bzw. $P_{c}^{\prime}$, deren perspektive Durchschnitte $b$ bzw. $c$ sind, und mithin der Schnittpunkt $\Lambda$ von $P_{b}^{\prime} L_{b}$ und $P_{c}^{\prime} L_{c}$ eine zu $K(l)$ projektive krumme Punktreihe $x^{2}(\Lambda)$ auf $\operatorname{dem} \operatorname{durch} A$ gehenden Kegelschnitt $x^{2}$ (Nr.90); alsdann wird also die $A$ von $A$ aus projizierende Gerade einen zu $K(l)$ projektiven Strahlenbüschel um $A$ beschreiben, und folglich wird auch die von $Q_{l}$, dem von $A \Lambda$ durch $P_{a}$ und $P_{a}^{\prime}$ harmonisch getrennten Punkte, beschriebene Punktreihe $p\left(Q_{l}\right)$ und somit auch die Kegelschnittschar $\mathfrak{f}\left(\Omega_{2}\right)$ zu $K(l)$ projektiv sein müssen; da jeder Kegelschnitt $\mathfrak{\Omega}^{2}$, der aus einer 
Geraden $l$ von $K$ hervorgeht, nach Nr. 95 von der Grundtangente $p$ in dem jener Geraden $l$ entsprechenden Punkte $Q_{l}$ berührt werden muß.]

Ist nun $K$ einer der Rückkehrpunkte von $P^{3}$, so liegen alle drei weiteren (außer $A$ ) gemeinsamen Punkte von $p^{2}$ und $x^{2}$ unendlich benachbart (Satz 41 in Nr.89), und zwar in einer der Ecken von $A B C$ (Nr. 93), und die Seiten des Dreiecks dieser drei unendlich benachbarten Punkte, von welchen Seiten zwei unendlich benachbarte Tangenten von $p^{2}$ in einem der Ecken von $A B C$ sind, müssen drei unendlich benachbarte Tangenten eines jeden aus einer Geraden $l$ von $K$ hervorgehenden Kegelschnitts $\mathfrak{Q}^{2}$ sein. Die Schar der Kegelschnitte $\mathfrak{2}^{2}$, welche aus den sämtlichen durch einen Rückkehrpunkt von $P^{3}$ gehenden Geraden $l$ hervorgehen, hat daher $p$ und noch drei einander unendlich benachbarte Geraden, von welchen letzteren zwei $p^{2}$ in einer der Ecken von $A B C$ tangieren, zu Grundtangenten. Jeder Kegelschnitt dieser Schar hat nun mit $p^{2}$ noch zwei weitere Tangenten gemein, welche bekanntlich eine Tangenteninvolution um $p^{2}$ bilden müssen. Die Achse dieser Tangenteninvolution muß, wenn der Rückkehrpunkt etwa $\mathfrak{H}_{A}$, der des Rückkehrstrahles $p_{A} \equiv P A$ von $P^{3}$, ist, die Dreieckseite $a \equiv B C$ sein. Denn derjenige Kegelschnitt $\mathfrak{Q}^{2}$, welcher aus der die beiden Rückkehrpunkte $\mathfrak{H}_{A}$ und $\mathfrak{u}_{B}$ verbindenden Geraden $l$ hervorgeht, muß (nach Satz 45 in Nr.99 und $I$ in Nr.94) von $p^{2}$ außer in $A$ noch in $B$ berührt werden, und mithin muß $B$ auf der Achse jener Tangenteninvolution liegen, ebenso aber auch $C$.

Mithin haben wir:

Satz 49. Ist $\mathfrak{l}_{A}$ der Rückkehrpunkt des Rückkehrstrahles $p_{A} \equiv P A$ von $P^{3}$, und sind $p_{1}$ und $p_{2}$ zwei veränderliche Strahlen von $P^{3}, t_{1}$ und $t_{2}$ die diesen Strahlen in der im Satze 48 angegebenen Weise entsprechenden Tangenten von $p^{2}$, so liegen die Berührungspunkte je zweier solcher Strahlen $p_{1}$ und $p_{2}$ von $P^{3}$ und nur solcher zwei Strahlen mit $\mathfrak{U}_{A}$ in je einer Geraden, deren entsprechende Tangenten $t_{1}$ und $t_{2}$ von $p^{2}$ sich auf der Dreieckseite $a$ schneiden.

Die Strahlenpaare von $P^{3}$, deren Berührungspunkte mit $\mathfrak{U}_{A}$ in je einer Geraden liegen, werden also von $p$ in den Punktepaaren derjenigen Involution geschnitten, deren Doppel- 
punkte $P_{b}^{\prime} \equiv p p_{B} \equiv(p, P B)$ und $P_{c}^{\prime} \equiv p p_{c} \equiv(p, P C)$ sind; und dual für $p^{3}$.

Dieser Satz ist im Satze 48 als Spezialfall enthalten, denn, wenn $\mathfrak{U}_{r} \equiv \mathfrak{U}_{A}$ ist, also $p_{r} \equiv p_{A}$, so ist $Q_{r} \equiv P_{a}^{\prime}, Q_{r}^{\prime} \equiv P_{a} \equiv p a, q_{r}^{\prime} \equiv a$, $U_{r}^{\prime} \equiv a p_{A}$ (Nr.2) und $u_{r}^{\prime}{ }^{2}$ artet (nach Nr.2) in das Geradenpaar $A P_{a}$ und $a$ aus, und dann wird stets, weil $A P_{a}$ Tangente an $p^{2}$ in $A$ ist (Nr.2), eine Seite von jedem $p^{2}$ um - und dem in $A P_{a}$ und $a$ ausgearteten Kegelschnitt eingeschriebenen Dreieck $A P_{a}$ selbst sein.

Ferner haben wir nun nach I (Nr. 94):

Satz 50. Ist $K$ irgendein ganz beliebiger Punkt, aber kein Berührungspunkt von $P^{3}, \varkappa^{2}$ der aus dem Polarkegelschnitt $k^{2}$ von $K$ (in bezug auf $A B C$ ) abgeleitete, etwa durch $P_{b}^{\prime}$ und $P_{c}^{\prime}$ gehende Kegelschnitt, $x_{1}, x_{2}, x_{3}$ die Seiten des Dreiecks der drei weiteren (außer $A$ ) voneinander verschiedenen (nach Satz 41 in Nr. 89) gemeinsamen Punkte von $x^{2}$ und $p^{2}, \mathfrak{f}\left(\Omega_{2}\right)$ diejenige Kegelschnittschar, deren Grundtangenten die vier ( $p^{2}$ nicht tangierenden) Geraden $p, x_{1}, x_{2}, x_{3}$ sind, $\mathfrak{l}^{3}$ diejenige Kurve dritter Ordnung, auf der bekanntlich ${ }^{1}$ ) die Eckpunkte der sämtlichen aus den je vier gemeinsamen Tangenten von $p^{2}$ und der Kegelschnitte der Schar $\mathfrak{f}\left(\mathfrak{Q}^{2}\right)$ gebildeten vollständigen Vierseite liegen, so liegen die Berührungspunkte von je vier solchen Strahlen $p_{1}, p_{2}, p_{3}, p_{4}$ von $P^{3}$ und nur von solchen vier mit $K$ in je einer Geraden, deren entsprechende (Satz 48) Tangenten $t_{1}, t_{2}, t_{3}, t_{4}$ von $p^{2}$ zugleich auch einen und denselben Kegelschnitt der Schar $\mathfrak{f}\left(\mathfrak{L}^{2}\right)$ tangieren und also ein solches vollständiges Vierseit bilden, dessen sechs Eckpunkte auf $\mathfrak{k}^{3}$ liegen.

Die Strahlenquadrupel von $P^{3}$, deren Berührungspunkte mit $K$ in je einer Geraden liegen, werden daher von $p$ in den Punktequadrupel einer biquadratischen Involution geschnitten; und dual für $p^{3}$.

Nunmehr sind die Sätze 48 und 49 als Spezialfälle in diesem Satze enthalten; denn, wenn $K$ ein Berührungspunkt $\mathfrak{H}_{r}$, von $P^{3}$ ist und dann $p^{2}$ von einer der Grundtangenten der Schar $\mathfrak{f}\left(\Omega^{2}\right)$

1) Vgl. Steiner-Schröter, Synthetische Geometrie, T. II, § 63, Nr. 346. 
tangiert wird, muß $\mathfrak{k}^{3}$, wie man sofort einsieht, in jene Grundtangente und den in Satz 48 angegebenen Kegelschnitt $u_{r}^{\prime 2}$ zerfallen, und wenn $K$ der Rückkehrpunkt $\mathfrak{H}_{A}$ von $P^{3}$ ist und dann $p^{2}$ in $A$ von zwei in $A P_{a}$ unendlich benachbart liegenden Grundtangenten der Schar $\mathfrak{f}\left(\Omega^{2}\right)$ tangiert wird, muß $\mathfrak{k}^{3}$ in die doppelt zu zählende Gerade $A P_{a}$ und die Dreieckseite $B C$ zerfallen.

Bemerkung. Die einem jeden Punkte $K$ durch die von $P^{3}$ eingehüllte Kurve vierter Ordnung in der angegebenen Weise mittels der Kegelschnittschar $\mathfrak{f}\left(\Omega^{2}\right)$ und $p^{2}$ zugeordnete Kurve dritter Ordnung $\mathfrak{k}^{3}$ muß stets $A B C$ umschrieben sein. Denn derjenige Kegelschnitt $\mathfrak{Q}^{2}$, welcher aus der $K$ mit dem Rückkehrpunkte $\mathfrak{U}_{A}$ verbindenden Geraden $l$ hervorgeht, wird von $p^{2}$ in $A$ berührt; mithin schneiden sich in $A$ zwei unendlich benachbarte gemeinsame Tangenten jenes Kegelschnitts $\mathfrak{Z}^{2}$ der Schar $\mathfrak{l}^{\left(\mathfrak{Z}^{2}\right)}$ und $p^{2}$, und folglich muß $A$ ein Punkt von $\mathfrak{k}^{3}$ sein; ebenso aber müssen auch $B$ und $C$ Punkte von $\mathfrak{k}^{3}$ sein. Wenn also $K$ eine gerade Punktreihe $l$ durchläuft, so muß die $K$ zugeordnete Kurve dritter Ordnung $\mathfrak{k}^{3}$ einen Kurvenbüschel beschreiben. In der Tat muß der aus der festen Geraden $l$ hervorgehende Kegelschnitt $\&^{2}$ in jeder der Kegelschnittschare $\mathfrak{f}\left(\mathfrak{L}^{2}\right)$, die den Punkten $K$ von $l$ zugehören, enthalten sein, und mithin müssen die sechs Eckpunkte des aus den vier gemeinsamen Tangenten dieses Kegelschnitts $2^{2}$ und $p^{2}$ gebildeten vollständigen Vierseits auf jeder der Kurven $\mathfrak{ł}^{3}$, welche den Punkten $K$ von $l$ zugeordnet sind, liegen; folglich müssen diese Kurven, die jene sechs Eckpunkte und außerdem noch die Eckpunkte von $A B C$ gemein haben, einen Büschel von Kurven dritter Ordnung bilden.

104. Aus I in Nr. 94 und Nr. 95 (dualisiert) ergeben sich unmittelbar die folgenden Lösungen der

Aufgabe 25. Die vier aus einem gegebenen Punkte $K$ an $p^{3}$ gehenden Tangenten zu ermitteln.

Auflösung 1. Man ermittelt die gemeinsamen Punkte $U_{q}, U_{r}, U_{s}, U_{t}$ des Polarkegelschnitts $P^{2}$ von $p$ (der Polare von $P$ bezüglich $A B C^{\prime}$ ) und desjenigen Kegelschnitts $\mathfrak{k}^{2}$, der durch $P, p_{A}^{\prime} k_{A}, p_{B}^{\prime} k_{B}, p_{C}^{\prime} k_{C}$ hindurchgeht und in $P$ von $g_{K}$ (der vom Schnittpunkte $a\left(p_{B}^{\prime} k_{B}, p_{C}^{\prime} k_{C}\right)$ durch $p_{A}$ und $p_{A}^{\prime}$ harmonisch getrennten Geraden) tangiert wird (wobei $k_{A} \equiv K A, k_{B} \equiv K B, k_{C} \equiv K C, p_{A} \equiv P A, p_{A}^{\prime} \equiv P P_{a} \equiv(P, p a)$, 
$p_{B}^{\prime} \equiv P P_{b} \equiv(P, p b), p_{C}^{\prime} \equiv P P_{c} \equiv(P, p c)$ sind $)$, und die Tangenten $q_{q}, q_{r}, q_{s}, q_{t}$ von $P^{2}$ in $U_{q}, U_{r}, U_{s}, U_{t}$; bringt sodann die Geraden $\left(B, p_{B}^{\prime} q_{q}\right)$ und $\left(C, p_{C}^{\prime} q_{q}\right)$ zum Schnitt im Punkte $P_{q},\left(B, p_{B}^{\prime} q_{r}\right)$ und $\left(C, p_{C}^{\prime} q_{r}\right)$ in $P_{r},\left(B, p_{B}^{\prime} q_{s}\right)$ und $\left(C, p_{C}^{\prime} q_{s}\right)$ in $P_{s},\left(B, p_{B}^{\prime} q_{t}\right)$ und $\left(C, p_{C}^{\prime} q_{t}\right)$ in $P_{t}$; alsdann werden die Geraden $K P_{q}, K P_{r}, K P_{s}, K P_{t}$ die vier von $K$ an $p^{3}$ gehenden Tangenten sein, und zwar werden sie $p^{3}$ in bzw. $P_{q}, P_{r}, P_{s}, P_{t}$ berühren.

Auflösung 2. Man ermittelt die gemeinsamen Tangenten $q_{q}^{(1)}, q_{r}^{(1)}, q_{s}^{(1)}, q_{t}^{(1)}$ der nämlichen Kegelschnitte $P^{2}$ und $\mathfrak{f}^{2}$, und bringt die Geraden $\left(B, p_{B}^{\prime} q_{q}^{(1)}\right)$ und $\left(C, p_{C}^{\prime} q_{q}^{(1)}\right)$ zum Schnitt in $P_{q}^{(1)},\left(B, p_{B}^{\prime} q_{r}^{(1)}\right)$ und $\left(C, p_{C}^{\prime} q_{r}^{(1)}\right)$ in $P_{r}^{(1)},\left(B, p_{B}^{\prime} q_{s}^{(1)}\right)$ und $\left(C, p_{C}^{\prime} q_{s}^{(1)}\right)$ in $P_{s}^{(1)},\left(B, p_{B}^{\prime} q_{t}^{(1)}\right)$ und $\left(C, p_{C}^{\prime} q_{t}^{(1)}\right)$ in $P_{t}^{(1)}$; alsdann werden die Geraden $K P_{q}^{(1)}, K P_{r}^{(1)}, K P_{s}^{(1)}, K P_{t}^{(1)}$ die vier aus $K$ an $p^{3}$ gehenden Tangenten sein, und zwar werden dann $P_{q}^{(1)}, P_{r}^{(1)}, P_{s}^{(1)}, P_{t}^{(1)}$ nicht die Berührungspunkte, sondern die weiteren (dritten) Schnittpunkte dieser Tangenten mit $p^{3}$ sein.
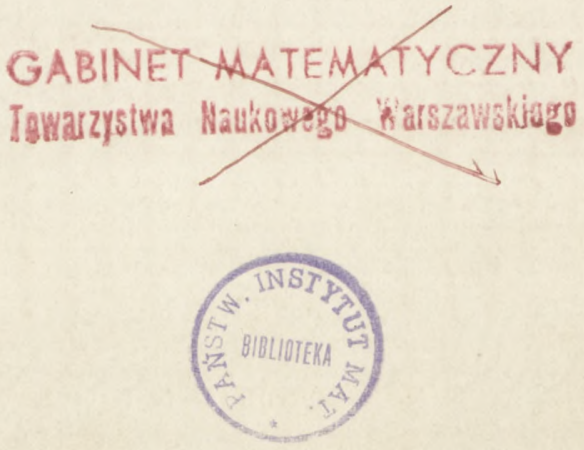
